\title{
Genetic engineering of microalgae for enhanced lipid production
}

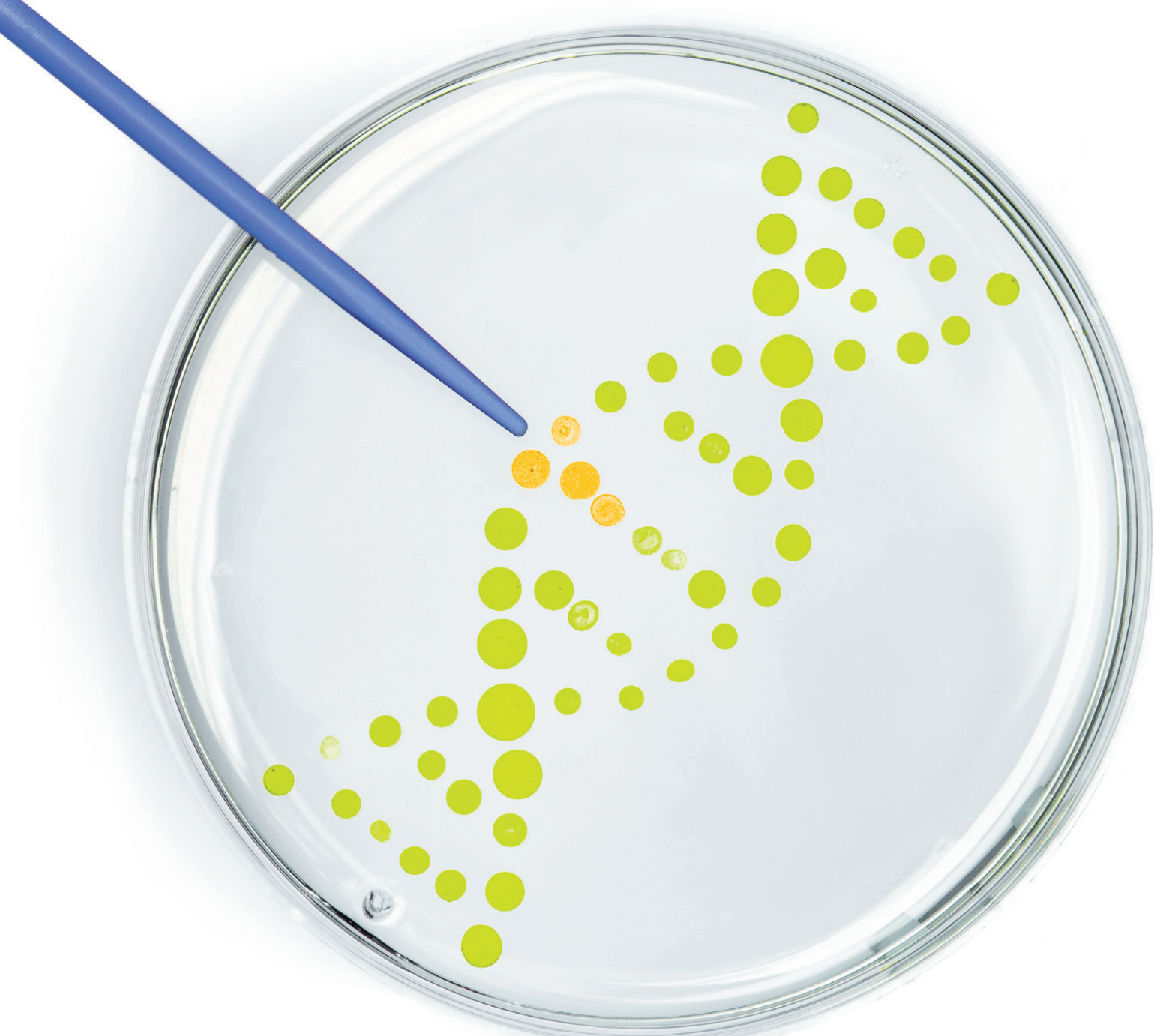

Camilo F. Muñoz Segovia 


\section{Propositions}

1. Deep understanding is needed to achieve efficient engineering of microalgae. (this thesis)

2. Microalgae are different from plants.

(this thesis)

3. The amount of scientific information available nowadays would have driven Leonardo da Vinci crazy.

4. Communicating science is more important than science itself.

5. Artificial intelligence enhances biased opinions.

6. A good photographer is a good storyteller.

Propositions belonging to the thesis, entitled

Genetic engineering of microalgae for enhanced lipid production

Camilo F. Muñoz Segovia

Wageningen, 26 October 2021 
Genetic engineering of microalgae for enhanced lipid production

Camilo F. Muñoz Segovia 


\section{Thesis Committee}

\section{Promotors}

Prof. Dr R. H. Wijffels

Professor of Bioprocess Engineering

Wageningen University \& Research

Prof. Dr R. A. Weusthuis

Personal chair at Bioprocess Engineering

Wageningen University \& Research

\section{Co-promotor}

Dr S. D' Adamo

Assistant Professor, Bioprocess Engineering

Wageningen University \& Research

\section{Other members}

Prof. Dr J. van der Oost, Wageningen University \& Research

Prof. Dr I. van Bogaert, Ghent University, Belgium

Dr C. Francke, HAN Biocentre, Arhnem-Nijmegen

Dr M. Suarez-Diez, Wageningen University \& Research

This research was conducted under the auspices of Graduate School VLAG (Advanced studies in Food Technology, Agrobiotechnology, Nutrition and Health Sciences). 


\title{
Genetic engineering of microalgae for enhanced lipid production
}

\author{
Camilo F. Muñoz Segovia
}

Thesis

submitted in fulfilment of the requirements for the degree of doctor at Wageningen University by the authority of the Rector Magnificus,

Prof. Dr A.P.J. Mol, in the presence of the

Thesis Committee appointed by the Academic Board to be defended in public on Tuesday 26 October at 4 p.m. in the Aula. 
Camilo F. Muñoz Segovia

Genetic engineering of microalgae for enhanced lipid production.

212 pages.

PhD thesis, Wageningen University, Wageningen, The Netherlands (2021) With references, with summary in English

ISBN: 978-94-6395-903-2

DOI: https://doi.org/10.18174/550437 


\section{Table of contents}

$\begin{array}{lll}\text { Chapter } \mathbf{1} & \text { Introduction and thesis outline } & \mathbf{1}\end{array}$

$\begin{array}{llll}\text { Chapter } 2 & \text { Improved } & \text { DNA/protein delivery in } & 19\end{array}$

microalgae - a simple and reliable method for the prediction of optimal electroporation settings

Chapter 3 Stable transformation of the green algae Acutodesmus obliquus and Neochloris oleoabundans based on E. coli conjugation

Chapter 4 Effect of single and combined expression of $\angle P A T, G P A T$ and DGAT on lipid accumulation and composition in Neochloris oleoabundans enhanced lipid production
Chapter 5 Genetic engineering of microalgae for General Discussion

Summary

References 



\section{Chapter 1}

Introduction and thesis outline 


\section{General introduction}

The continuously growing world population is rapidly increasing the global demand for food, feed and fossil-based products. Moreover, excessive and unsustainable consumption cause elevated environmental pollution levels and depletion of natural resources. The major feedstocks used for food, feed and fuel production are mainly derived from agricultural crops and non-renewable fossil reserves. Higher plants have traditionally been applied for the production of vegetable oil used in food, paint and lighting applications. More recently, vegetable oil has also been applied for fuel purposes, especially in the form of biodiesel. This, however, creates a direct competition with food and feed production. Additionally, crop cultivation for oil production requires vast areas of arable land and high amounts of fresh-water. Therefore, renewable and sustainable alternatives are required to reduce the current environmental impact of traditional industrial feedstocks.

Microalgae are considered as promising biological platforms for the production of numerous valuable products (Chisti, 2007; Christaki et al., 2011; Draaisma et al., 2013; Gimpel et al., 2013; Pulz \& Gross, 2004; Wijffels et al., 2013; Wijffels \& Barbosa, 2010). These photosynthetic microorganisms are capable of converting sunlight, water, $\mathrm{CO}_{2}$ and minor other nutrients into biomass. Unlike higher plants, they do not require arable land for their cultivation, they can reach significantly higher biomass concentrations and product yields per hectare, and some species do not require fresh water as they are able to grow on wastewater or seawater (Brennan \& Owende, 2010; Griffiths \& Harrison, 2009; Medipally et al., 2015; Remmers et al., 2018). Several microalgal species cultivated under stress conditions can accumulate large amounts of fatty acids which can serve either as cellular structural components or storage compounds. Oleaginous microalgae strains such as Neochloris oleoabundans can produce TAGs up to $44 \%$ of their dry weight (Breuer et al., 2012). Furthermore, some microalgal species are able to produce high-value polyunsaturated fatty acids (PUFAs) such as omega- 3 and omega- 6 fatty acids (Ghiffary et al., 2019). 
Although microalgae are a promising source for edible oil and biodiesel production, some limitations and challenges remain. Current cultivation systems are limited to niche markets because they involve high production costs that do not allow commercial-scale production of such compounds. In order to achieve an economically feasible process, current photo-bioreactor systems require improvement, optimization and redesign. Moreover, costs can be reduced by optimization of cultivation conditions and selection of microalgae strains with higher lipid productivities, growth rates and photosynthetic efficiencies (Ruiz et al., 2016). Lastly, deeper understanding in lipid metabolism can accelerate the generation of strains with enhanced growth and lipid production through genetic engineering.

\section{Microalgal lipids}

Microalgae are a promising source of valuable fatty acids. They contain major lipid classes of great relevance in industry (Khan et al., 2018). Yet, the fatty acid composition and lipid profile of different species can vary significantly (Breuer et al., 2012). The major lipid classes found in microalgae are storage compounds in the form of triacylglycerols (TAGs) and membrane lipids such as glycosylglycerides, phosphoglycerides and betaine ether lipids. An overview of lipid metabolism in microalgae is presented in figure 1. Moreover, microalgae can also possess other lipids that are less common such as phosphatidylsulphocholine (found in diatoms and Euglena) (Harwood \& Jones, 1989), novel hydrocarbons (found in Botryococcus braunii) (Guschina \& Harwood, 2006) and halogenated fatty acids and their derivatives in other microalgae species. Although the lipid composition can vary significantly among microalgal species, it has been determined that the major component of thylakoids membranes include three glycosylglycerides: monogalactosyldiacylglycerol (MGDG), digalactosyldiacylglycerol (DGDG), and sulphoquinovosyldiacylglycerol (SQDG), with the latter being the most predominant constituent (Li-Beisson et al., 2016).

Membrane phospholipids derived from glycerol or phosphoglycerides can be found in minor amounts compared to glycosylglycerides. Their main 
constituents are phosphatidylcholine (PtdCho), phosphatidylethanolamine (PtdEtn) and phosphatidylglycerol (PtdGro) which have a key role as structural components.

Lastly, other membrane components are betaine lipids which can have an important role for allowing cells to adapt to low temperatures and phosphate deficiency as has been shown in Nannochloropsis species (Murakami et al., 2018). To date, three betaine lipids have been identified in microalgae such a diacylglyceryl- $N, N, N$-trimethylhomo-serine (DGTS) which are the most common in nature and found in green algae, diacylglyceryl-hydroxymethyl$N, N, N$-trimethyl- $\beta$-alanine (DGTA) found in brown algae and diacylglycerylcarboxylhydroxymethylcholine (DGCC) found in algae strains of the genus Haptophyceae such as Pavlova luthera (Canãvate et al., 2016; Eichenberger, 1993; Kato et al., 1996).

Among neutral lipids that can be found in microalgal cells, the most common saturated fatty acids are 14:0, 16:0 and 18:0 (Zulu et al., 2018). Moreover, the most abundant neutral lipids found as storage compounds are monoacylglycerols, diacylglycerols and triacylglycerols, the latter being the predominant species in lipid bodies. TAG biosynthesis is commonly induced by nutrient limitation such as nitrogen starvation (Janssen et al., 2018). They are mainly composed by saturated or monounsaturated fatty acyl groups which are of great interest in industry for their potential use as source for biofuel production (Brennan \& Owende, 2010).

Other commercially important fatty acids are very long chain polyunsaturated fatty acids (VLC-PUFAs) such as eicosapentaenoic (EPA), docosahexaenoic (DHA) and arachidonic acid (ARA) which are mainly found in marine or salttolerant microalgal species (Guschina \& Harwood, 2006; Harwood \& Jones, 1989). 


\section{Enhancing lipid production via genetic engineering in microalgae}

Advances in biotechnology field including genetic engineering, genome editing techniques and omics technology have allowed a more affordable use of technology such as sequencing, which have increased the number of genomes available (W. R. Lin et al., 2019). Moreover, the use of advanced bioinformatic tools have allowed a deep understanding of algal lipid metabolism. Key enzymes and lipid biosynthesis pathways have been elucidated enabling genetic engineering of microalgae to improve lipid production. The first in silico analysis of lipid synthesis in algae was achieved in Chlamydomonas reinhardtii which allowed the reconstruction of the fatty acid synthetic pathway (Moellering et al., 2009; Riekhof et al., 2005). Subsequently, several genetic engineering strategies to increase lipid content have been proposed and tested successfully in different microalgal species (Foo et al., 2019; Ghiffary et al., 2019).

The first attempt to enhance fatty acids and TAG synthesis was performed in Cyclotella cryptica and Navicula saprophila (Terri G. Dunahay et al., 1996). The primary target of this study was to increase the expression of genes encoding for acetyl-CoA carboxylase (ACCase) which mediates the key rate-limiting step in fatty acid synthesis (figure $\mathbf{1}$ ). In this study, although a significant augmentation in ACCase activity was observed, the engineered strains did not show increased fatty acid synthesis. However, later experiments showed that simultaneous overexpression of genes encoding ACCase and malic enzyme could successfully enhance lipid production in Dunaliella salina (Farhad Talebi et al., 2014). The most successful approach applied to increase total lipid and triacylglycerol content is the overexpression of genes encoding for enzymes involved in the Kennedy pathway (Naghshbandi et al., 2020). This includes overexpression of genes encoding glycerol-3-phosphate acyltransferase (GPAT) (Balamurugan et al., 2017), lysophosphatidic acid acyltransferase (LPAT) (X. Wang, Dong, et al., 2018a), phosphatidic acid phosphatase (PAP) (X. D. Deng et al., 2013) and diacylglycerol acyltransferase (DGAT) (Dinamarca et al., 2017) (figure 1). DGAT catalyzes the final step in the TAG biosynthesis 
pathway (Naghshbandi et al., 2020; Snyder et al., 2009) and thus is a key target for increasing lipid content in microalgae. On the other hand, genetic engineering strategies for enhanced PUFA production such as EPA and DHA have been achieved by overexpressing genes encoding desaturases or elongases such as $\Delta 5$-DES, $\Delta 12$-DES or $\Delta 6$-DES and $\Delta 5$-EL in strains such as

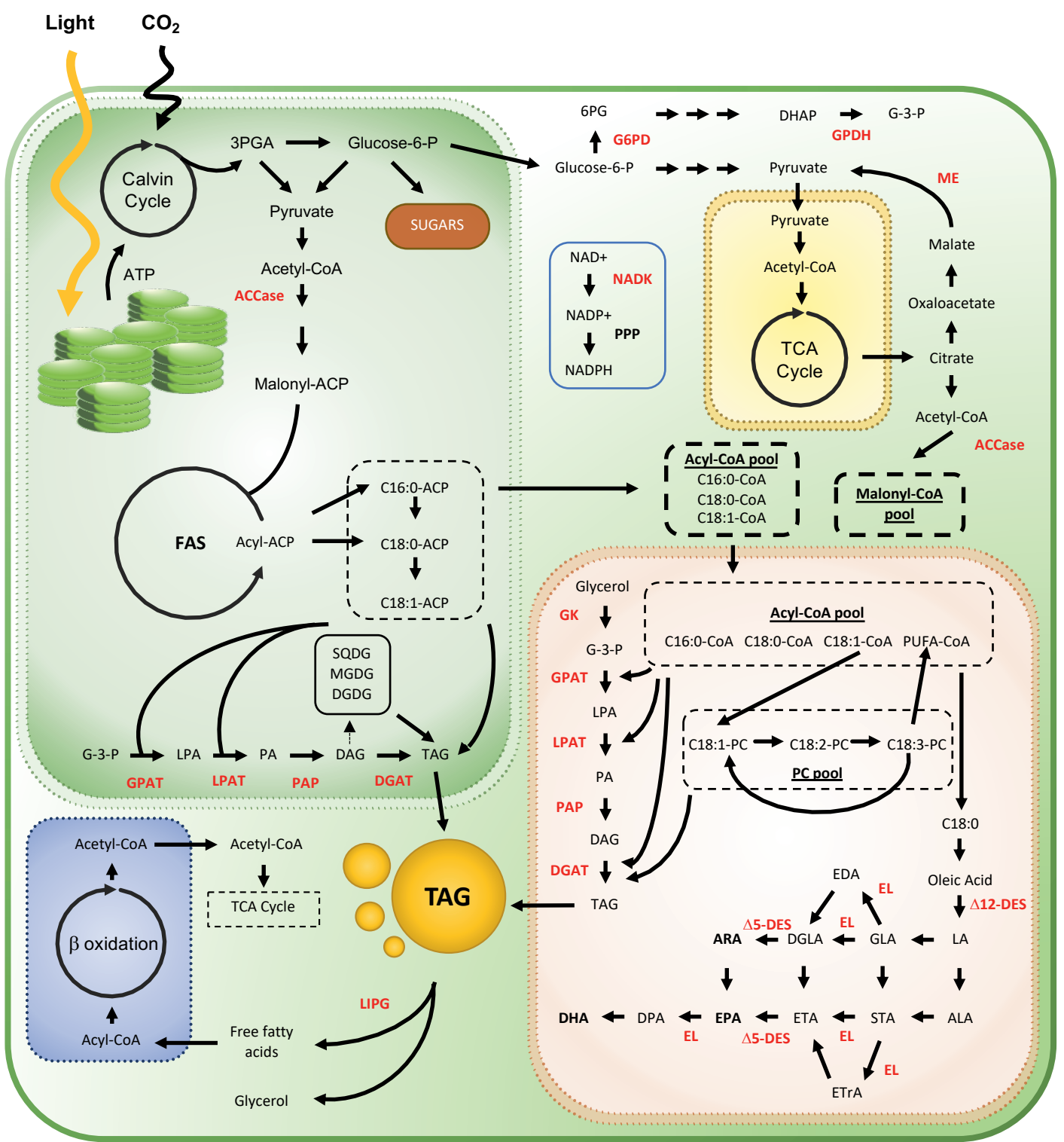

Figure 1. Overview of lipid biosynthesis pathways in microalgae. Pathways and metabolites are shown in black. Red annotations represent enzymes. Green, yellow, blue and orange boxes represent chloroplast, mitochondria, peroxisome and endoplasmic reticulum, respectively. 
Nannochloropsis gaditana and Thalassiosira pseudonana (Cook \& Hildebrand, 2016; Poliner et al., 2018).

Other promising strategies to enhance lipid production have targeted competing pathways such as lipogenesis and lipid catabolism (Kong et al., 2018). Several strains are able to synthesize large amounts of sugars such as starch as their primary storage metabolite (Ran et al., 2019). Therefore, inhibition of starch biosynthesis can be performed to redirect carbon flux towards lipid production. This has been demonstrated in the starchlessmutants of Chlamydomonas reinhardtii and Scenedesmus obliquus which have increased their total lipid content per cell during nitrogen starvation (De Jaeger et al., 2014; Yantao Li et al., 2010). Furthermore, suppressing or reducing activity of lipases can decrease the rate of lipid catabolism and thus increase total lipid content. However, this approach has shown to have a negative impact on microalgal growth and biomass production (Chu, 2017). This can be overcome by controlling the reduced gene expression using RNA or CRISPR interference which could maintain a basal expression level thereby decreasing the impact on growth (Y.-T. Zhang et al., n.d.). In addition, inhibition of $\beta$ oxidation has also proven to be a favorable target for increased lipid content. For instance, a mutation in the $A C X$ gene encoding acyl-CoA oxidase resulted in low lipid degradation which increased the oil content in Chlamydomonas reinhardtii under nitrogen-limited conditions (Kong et al., 2017).

Modification of regulatory signaling pathways is an alternative strategy to regulate multiple genes simultaneously in a metabolic pathway. Several transcriptional factors and transcription-related proteins have been identified in microalgae that can be used as potential targets to shift metabolic flux towards lipid biosynthesis. Overexpression of transcriptional factors such as BHLH, WRI1, Dof-type TF and NobZIP1 have shown to increase lipid content compared to wild type strains (Ibáñez-Salazar et al., 2014; N. K. Kang et al., 2015, 2017b; D. W. Li et al., 2019). Furthermore, attenuating expression of negative regulators of cellular lipid synthesis such as $\mathrm{Zn}(\mathrm{II})_{2} \mathrm{Cys}_{6}$ TF has resulted in higher lipid productivities as well (Ajjawi et al., 2017). Overall, transcriptional factors have provided advantages over traditional strategies due 
to their ability to up or down regulate entire pathways involved in lipid biosynthesis pathways, cell growth, development and stress-related behaviors (Thiriet-Rupert et al., 2016).

Additionally, manipulation of metabolic pathways which are not directly involved in lipid synthesis have demonstrated positive effects on fatty acid accumulation. For instance, increasing photosynthetic efficiency provides extra reducing energy to power biosynthetic pathways and enhance carbon fixation which results in higher lipid synthesis. This approach has shown promising results when targeting light-harvesting antenna complexes (de Mooij et al., 2015; Jeong et al., 2017; Kirst et al., 2012) or increasing ribulose-1,5bisphosphate carboxylase/oxygenase (Rubisco) activity which is the main enzyme responsible for carbon assimilation in the Calvin Cycle. Overexpression of Rubisco has been done in Nannochloropsis oceanica resulting in an increased biomass and lipid content (L. Wei et al., 2017).

Another indirect strategy is to enhance redox cofactors generation in the cell which are required in anabolic processes such as lipid synthesis. For instance, NADPH supply has been increased in Chlamydomonas reinhardtii by overexpressing malic enzyme (ME), which catalyzes the conversion of malate to pyruvate and releases NADPH in the process (Kim et al., 2019). Increased NADPH supply has also been demonstrated by overexpression of genes encoding glucose-6-phosphate dehydrogenase (G6PD), glycerol-3-phosphate dehydrogenase (GPDH) and NAD kinase (NADK) (figure 1). This approach has successfully increased lipid content in strains such as Chlorella spp., Phaeodactylum tricornutum and Chlorella pyrenoidosa (Fan et al., 2015; Xue et al., 2017; Xue et al., 2020; Yao et al., 2014).

Genetic engineering strategies have proven to increase lipid content over the last decade. However, the development of transformation methods and a robust genetic toolbox are still not available to all relevant strains. Moreover, limited information is available regarding gene regulation as a response to different environmental conditions and further research is needed to unravel relevant metabolic pathways in industrially promising species. Developments 
in genetic engineering combined with systems biology will enable the study of gene functions and creation of strains with desired traits. The generation of strains with an enhanced lipid metabolism will allow a more sustainable and competitive use of microalgae as source of fatty acids.

\section{Strain engineering and tool development}

In the last decades, considerable progress has been made in the development of genetically improved strains with an enhanced metabolism towards biomass and product formation. Genetic modification of promising microalgae strains has been achieved by adaptive laboratory evolution, random mutagenesis and direct genetic engineering. Moreover, recent advances in genomics, transcriptomics, proteomics, metabolomics, and bioinformatics have allowed to increase our understanding of fatty acid biosynthesis pathways. Therefore, the development of genetic tools and techniques for the modification of microalgae has become a promising approach for the generation of improved strains with enhanced lipid productivities. Below, the main genetic tools used for strain improvement are described.

Classical mutagenesis and adaptive laboratory evolution. Different physical and chemical mutagens have been used to induce mutations in the genome at a higher rate than naturally occurring in a particular organism. UV light, gamma radiation and $\mathrm{X}$-rays as well as chemical mutagens such as $\mathrm{N}^{\prime}$ nitro-N-nitrosoguanidine (NTG) and ethyl methanesulfonate (EMS) have been successfully applied on microalgae (Chou et al., 2019; B. Lee et al., 2014). Ultraviolet light UVA and UVB have shown to induce formation of pyrimidine dimers on DNA strands (Ikehata \& Ono, 2011). On the other hand, X-rays and gamma rays have higher energy photons which results in higher penetration power, causing significant DNA damage including single and double strand breaks. The use of these electromagnetic (EM) spectra presents several advantages such as reduced contamination and easy control of its exposure to organisms compared to chemical mutagens. EM techniques have been used to improve both growth and lipid content in Phaeodactylum, Chlamydomonas and Scenedesmus strains (Cagnon et al., 2013; Sivaramakrishnan \& 
Incharoensakdi, 2017; Yi et al., 2015). NTG and EMS have been successfully used for the generation of mutants with enhanced lipid accumulation for instance in Chlamydomonas and Nannochloropsis (T. A. Beacham et al., 2015; B. Lee et al., 2014).

Another technique commonly applied to evolve microalgae strains is adaptive laboratory evolution (ALE) (X. M. Sun et al., 2018). Strains are subjected to environmental stress under well-controlled laboratory conditions which allows the generations of stress-adapted strains with desired phenotypes. Several selection parameters have been used such as growth rate, light stress, temperature, chemical treatment, nutrient limitation as well as antibiotics ( $X$. M. Sun et al., 2018).

Importantly, classical mutagenesis and ALE can cause many random mutations. Therefore, identification of mutants of interest requires the availability of high-throughput screening methods and sequencing.

Genetic tool development. Although classical mutagenesis has been successfully used for the creation of random mutations, a more directed approach to generate specific insertions, deletions or substitutions into the host strain is needed in order to achieve major changes in the microalgal metabolism. Genetic modification of microalgae is challenged by the limited genetic toolbox that is currently available. A broader range of genetic tools could offer a wider spectrum of capabilities allowing fast, accurate, efficient and more comprehensive development of algal cell factories.

Advances in sequencing technologies have allowed the availability of hundreds of genomes. Thousands of genes have been annotated and uploaded into databases. In addition, several studies have collected important information regarding transcriptomics and metabolomics. Recent developments in bioinformatic technologies have not only allowed to gain insights in lipid metabolism but also the identification of regulatory elements necessary for the development of genetic engineering strategies (Park et al., 2019). Identification of promoters, splicing signals, terminators, selection and reporter markers are essential for such an approach. It has been demonstrated that 
either inducible or constitutive expression of endogenous and heterologous genes can have a significant impact on product formation (Doron et al., 2016). Moreover, successful genetic engineering tools have been demonstrated to work in microalgae strains including mono/polycistronic expression systems, chloroplastic/nuclear gene integration and genome editing techniques such as CRISPR/Cas/Cpf1, TALENs, RNAi which can be used for gene knock-out, knockdown and knock-in strategies (W. R. Lin et al., 2019; Park et al., 2019; Y. T. Zhang et al., 2019). On the other hand, bioinformatic tools have allowed the construction of genome-scale metabolic models which can predict the effect of gene insertion, deletion or silencing involved in specific metabolic pathways (Lauritano et al., 2019; W. R. Lin et al., 2019; Y. T. Zhang et al., 2019).

Despite great improvement in genetic tool development, most of the advances are mainly restricted to the species Chlamydomonas, Phaeodactylum and Nannochloropsis. These species have become model organisms for physiology, photosynthesis and genetic engineering research in the last decades. Other industrially relevant oleaginous microalgae such as Chlorella vulgaris, Neochloris oleoabundans and Acutodesmus obliquus, have a great potential to become part of bio-based production platforms (Gimpel et al., 2015; Hannon et al., 2010). At the moment, however, the required genetic engineering tools for these strains are not available, especially in less well-studied microalgal species due to the lack of efficient DNA delivery techniques.

\section{DNA delivery into microalgae cells}

DNA delivery into microalgae cells is considered to be a major bottleneck due to the great variation in cell sizes, cell wall structures and composition between species (Azencott et al., 2007; Ortiz-Matamoros et al., 2018). Transformation techniques aim to transfer DNA molecules through the cell wall, plasma membrane and nuclear membrane using chemical or mechanical treatments. Microalgal transformation methods can require specialized equipment and they need optimization to avoid poor transformation efficiencies particularly when relatively long DNA fragments are delivered into the microalgae cells. In addition, most of the transformation methods can lead to high cell death since 
the cells are not able to recover after the treatment is applied. Therefore, particular methods are needed for specific strains, and a broader range of genetic tools have to be developed.

Among transformation methods for the delivery of exogenous DNA into microalgae cells, the most common and successful techniques are electroporation, Agrobacterium tumefaciens-mediated transformation (ATMT), particle bombardment and agitation with glass beads (Apt et al., 1996; T. G. Dunahay, 1993; Karen L. Kindle, 1998). Although most of these techniques have been proven to work with great success in model strains such as Chlamydomonas reinhardtii, Phaeodactylum tricornutum, there is a lack of efficient and stable transformation techniques that can be applied to a broader range of microalgae strains.

Electroporation. Electroporation is a widely used DNA delivery technique used to transfer exogenous DNA across membranes of microorganisms such as bacteria, yeast or plant protoplasts. This method is relatively simple, time efficient and inexpensive. An electric charge is applied creating temporary micro-pores in the cell membrane which function as a channel allowing highlycharged molecules such as DNA to diffuse passively across the membrane. Electroporation conditions such as electric field strength, electroporation buffer, capacitance, number of pulses or the use of enhancers have shown to have an effect on transformation efficiencies. Additionally, the use of cell wall-deficient strains has shown an improvement in transformation efficiencies (OrtizMatamoros et al., 2018). This method has been used successfully in a number of microalgae species including Chlamydomonas, Scenedesmus and Chlorella species (Chow \& Tung, 1999; Guo et al., 2013; Shimogawara et al., 1998).

Particle bombardment. Biolistics or particle bombardment is a common DNA delivery method used for plant transformation. This method utilizes gold or tungsten particles coated with DNA that are delivered at high velocity into the cells under vacuum conditions with a helium pressurized gene gun. A major advantage of this method is that DNA can overcome rigid physical barriers such as cell walls and can be used for DNA delivery in a number of compartments 
such as nucleus, chloroplast and mitochondria (Jinturkar et al., 2011). However, this method requires expensive equipment and its use can result in multiple copy insertions of DNA which can lead to unwanted recombination or silencing (Matsumoto \& Gonsalves, 2012). This technique has been successful in microalgae such as Chlamydomonas, Dunaliella and diatoms (Apt et al., 1996; Ramesh et al., 2011; C. Tan et al., 2005).

Glass beads. Glass bead-based transformation methods involve the agitation of microalgae strains in the presence of glass beads coated with DNA. This mechanical method creates micro-pores due to friction and collision between beads and cells, enabling the passage of DNA molecules into the cell. Although it has been a successful DNA delivery method, it usually requires the generation of protoplasts (cell wall-deficient mutants) or microalgae species that do not possess a thick cell wall, presence of polyethylene glycol (PEG) and the use of linearized DNA (K. L. Kindle, 1990). This method has shown to be successful for microalgae species such as cell wall-less Chlamydomonas reinhardtii (Economou et al., 2014; K. L. Kindle, 1990).

Agrobacterium-tumefaciens mediated transformation. Agrobacteriumtumefaciens transformation method has been widely used for exogenous DNA transfer into plants. Agrobacterium tumefaciens is a gram-negative bacterium that possesses the ability to induce the formation of tumors in higher plants by introducing a T-plasmid containing a set of genes needed for the integration of its genome into the host organism. The native mechanism has been modified by removing tumor-inducing genes and has been adopted as a vehicle for DNA transfer to other relevant organisms. The removal of virulence ( $v i r$ ) genes such as virC, virF and virH has allowed DNA transfer to a broad group of organisms such as plants, yeasts and microalgae (Hooykaas, 2004). Temperature, cocultivation period and donor/recipient ratios have shown to be important parameters to consider in order to achieve high transformation efficiencies (Uranbey et al., 2005). This method has been successfully used in microalgae species such as Chlorella and Ankistrodesmus species (Suttangkakul et al., 2019). 
Bacterial conjugation. Bacterial conjugation is a natural mechanism for gene transfer between bacterial strains. This mechanism has been extensively used as a transformation method for the delivery of cloning and expression vectors to prokaryotic cells. The transfer of genetic material via bacterial conjugation mainly requires an origin of transfer and mob genes which are essential for the DNA mobilization. Mob genes are needed for DNA transfer, they encode for enzymes involved in the recognition and catalysis of the cleavage at the origin of transfer site (oriT) which creates a single stranded DNA that can be transferred into recipient cells. Although this mechanism occurs naturally between bacteria, it has been shown to occur between prokaryotic and eukaryotic cells as well (Lacroix \& Citovsky, 2016). The main advantages over traditional methods include its simplicity, minimal disruption of algae cells, transfer of relatively large plasmids which allows expression of large set of genes and no need of expensive equipment. This transformation method has been successfully used in microalgae strains such as Thalassiosira pseudonana and Phaeodactylum tricornutum (Diner et al., 2016; Karas et al., 2015). 


\section{Aim and thesis outline}

The goal of this thesis is to develop a genetic toolbox for the transformation of oleaginous microalgal strains and use this technology for the generation of strains with enhanced lipid production. As introduced in Chapter 1, microalgal lipid production is not yet economically feasible and competitive compared to lipid-producing crops. Therefore, novel genetic engineering tools and strategies for strain improvement are needed to achieve this goal. The approach used in this thesis was the development of efficient transformation methods and overexpression of genes encoding key enzymes responsible for triacylglycerol production.

Transformation methods have been well established for model algae species. However, reliable and efficient transformation tools are needed for poorly transformable strains or less well-studied microalgal species. A quick and effective screening tool that can be used for the optimization of electroporation settings is described in Chapter 2. The proposed tool allows the analysis of cell permeability to DNA and proteins, and the effect of the electric pulse on cell viability by using fluorescent dyes. The optimization method presented in this chapter is a promising strategy which could be used as guideline to achieve successful DNA delivery into a variety of microalgal species.

Chapter 3 provides an alternative method for the delivery of exogenous DNA into microalgae cells. A conjugation-based method is proposed for the genetic transformation of green microalgae species. This approach offers advantages over other transformation methods such as reduced cellular damage, and allows the transfer of large plasmids with no need of expensive equipment. This conjugation-based transformation method is tested in both Acutodesmus obliquus and Neochloris oleoabundans.

A genetic engineering strategy to increase lipid content in $N$. oleoabundans is described in Chapter 4. The electroporation method established in Chapter 2 is used for DNA delivery into algal cells. Single and combined heterologous expression of genes involved in the Kennedy pathway are promising targets to 
increase the main lipid storage compound (TAGs). Genes encoding GPAT, LPAT and DGAT enzymes from the high-lipid producing strain $A$. obliquus are delivered into $N$. oleoabundans. Growth, lipid composition and cellular biochemical composition of transformant lines are investigated.

Chapter 5 provides an in-depth review on the generation of enhanced lipid producing strains via genetic engineering. Established and new insights in lipid biosynthesis pathways are described. Successful genetic engineering strategies and promising gene targets used over the last decades are presented. Manipulation of key genes involved in Kennedy pathway, regulatory signalling pathways, photosynthetic efficiency, carbon fixation, redox cofactor generation, lipogenesis and lipid catabolism are few examples of the approaches discussed to increase lipid production. Furthermore, new genetic tools and strategies are discussed and proposed for future development in the field.

Chapter 6 provides a general discussion of microalgal strain improvement strategies. The results obtained in this thesis are compared to those achieved by others. Furthermore, we discuss main challenges in technology and genetic tool development needed to create strains with high-lipid content able to compete with conventional oil sources. 



\title{
Chapter 2
}

\author{
Improved DNA/protein delivery in \\ microalgae - a simple and reliable method \\ for the prediction of optimal \\ electroporation settings
}

This chapter has been published as: Muñoz, C.F.*, de Jaeger, L.*, Sturme, M.H.J., Lip, K.Y.F., Olijslager, J.W.J., Springer, J., Wolbert, E.J.H., Martens, D.E., Eggink, G., Weusthuis, R.A., Wijffels, R.H. (2018). Improved DNA/protein delivery in microalgae - A simple and reliable method for the prediction of optimal electroporation settings. Algal Research, 33: 448-455.

* Contributed equally 


\section{Abstract}

Genetic transformation of microalgae remains a challenge due to poor intracellular delivery of exogenous molecules. This limitation is caused by the structure and composition of the cell wall and cell membrane of each species. Moreover, successful delivery of proteins or nucleic acids cannot be assessed by determining transformability since their functionality is not always known in the studied microorganism. We propose a quick and effective screening tool for the prediction and optimization of electroporation settings by monitoring cell permeability and viability using Sytox Green and propidium iodide respectively. We determined voltage settings for the microalgae Chlamydomonas reinhardtii, Chlorella vulgaris, Neochloris oleoabundans and Acutodesmus obliquus. To evaluate the predicted settings, we delivered labelled DNA and proteins into the cells. We demonstrated that high transformation efficiencies can be accomplished when predicted values were applied with functional plasmids. Additionally, we increased transformation efficiencies by testing cell concentrations, light intensities and fragment sizes. This method can be used to determine suitable transformation conditions for non-transformed microalgae species and to increase the insight on established transformation protocols.

\section{Keywords}

Electroporation, green microalgae, labelled DNA, labelled protein, Sytox Green, propidium iodide. 


\section{Introduction}

Microalgae provide a promising platform for the production of high value products such as nutraceuticals, commodity chemicals, health beneficial products, lipids and proteins for food and feed applications (Chisti, 2007; Christaki et al., 2011; Draaisma et al., 2013; Gimpel et al., 2013; Pulz \& Gross, 2004; Wijffels et al., 2013; Wijffels \& Barbosa, 2010). Wijffels and Barbosa (Wijffels \& Barbosa, 2010) described the physiological and cellular characteristics of an "ideal microalgal cell factory" and even though extensive screening studies of microalgal isolates have been done, it is unlikely that single species can meet all required characteristics, such as high growth rate, high productivity of valuable compounds, product excretion and robustness. Genetic modification and metabolic engineering are valuable tools to speed up the development of microalgae derived products and are necessary to decrease the high production costs that are currently involved.

Methods have been developed to enable delivery of exogenous DNA into microalgal cells, such as particle bombardment, Agrobacterium tumefaciens mediated transformation, conjugation, electroporation, and methods involving glass beads and carbon whiskers (Apt et al., 1996; T. G. Dunahay, 1993; Karen L. Kindle, 1998). Additionally, delivery of preassembled recombinant Cas9/Cpf1 ribonucleoproteins (RNPs) via electroporation has enabled genome editing in microalgae (Baek et al., 2016). Most advances are restricted to the species Chlamydomonas reinhardtii which has been a model for photosynthesis and metabolic engineering research for many decades (Merchant et al., 2007). Although Chlamydomonas species have been well characterized, they are not well suited for producing high value compounds. Industrially relevant oleaginous microalgae such as Chlorella vulgaris, Nannochloropsis sp., Neochloris oleoabundans and Acutodesmus obliquus, have a great potential to become part of bio-based production platforms (Gimpel et al., 2015; Hannon et al., 2010). However, these promising species still need to be further optimized, for which a genetic engineering toolbox that can be applied to a wide range of microalgal species is required. 
The most widely used method to deliver exogenous molecules such as DNA and proteins into cells is electroporation (Chow \& Tung, 1999; Kilian et al., 2011; Shimogawara et al., 1998). This physical method is based on electropermeabilization, in which an electric pulse generates temporary micropores in the cell membrane enabling the passage of molecules into the cells. This passage can be obstructed by the cell wall, or shape and size of the cells (Allard et al., 1998). Since the main cause for unsuccessful delivery of molecules into microalgae is related to the morphology of a specific species (Azencott et al., 2007; Ortiz-Matamoros et al., 2018), all electroporation conditions such as electric field strength, electroporation buffer, capacitance, number of pulses or the use of enhancers have to be optimized for each species individually. However, optimal settings cannot be investigated by determining the amount of transformants obtained after electroporation since it is not always known if the expression vector to be delivered is functional in the investigated microorganism. We therefore propose a quick and effective screening tool that can be used for the optimization of electroporation settings needed for the delivery of exogenous molecules in poorly transformable strains or less well-studied microalgal species. We optimized the electroporation conditions and give guidelines for measuring cell permeability and viability by using two fluorescent dyes: Sytox Green and propidium iodide respectively. Furthermore, labelled DNA and labelled proteins were delivered into the cells to corroborate the results obtained during permeabilization experiments. Finally, we demonstrate successful microalgal transformation by using an optimized electroporation protocol. To generate a generic genetic engineering toolbox that can be applied to a wide range of microalgal species, we tested four industrially relevant microalgae species with different physiological and cellular characteristics: Chlamydomonas reinhardtii, Chlorella vulgaris, Neochloris oleoabundans and Acutodesmus obliquus.

This method not only offers guidance for finding optimal electroporation settings in species that are less prone to take up extracellular molecules, but it can also be used to improve performance and increase insight in established protocols. 


\section{Materials and Methods}

\section{$\underline{\text { Strains, medium and culture conditions }}$}

Chlamydomonas reinhardtii (CC1690, Chlamydomonas research center), Acutodesmus obliquus (UTEX 393, University of Texas), Chlorella vulgaris (UTEX 259, University of Texas) and Neochloris oleoabundans (UTEX 1185, University of Texas) were used in this study. C. reinhardtii was grown on TrisAcetate-Phosphate (TAP) medium (Gorman \& Levine, 1965). Acutodesmus obliquus, Chlorella vulgaris, and Neochloris oleoabundans were grown in fresh water medium (Breuer et al., 2012). Agar plates were made by supplementing $15 \mathrm{~g} / \mathrm{L}$ agar (Duchefa Biochemie B.V., Haarlem, The Netherlands). Cultures were kept in exponential growth phase under day:night cycle regime $(16: 8)$ at $25^{\circ} \mathrm{C}$ at around $60 \mu \mathrm{mol} \mathrm{m} \mathrm{m}^{-2} \mathrm{~s}^{-1}$ light intensity (Grolux fluorescent tubes, Sylvania F36W/GRO) at $125 \mathrm{rpm}$ and $2.5 \% \mathrm{CO}_{2}$.

E. coli $\mathrm{S} 17$ and $\mathrm{DH} 5$ a were grown in LB and LB agar at $37^{\circ} \mathrm{C}$ supplemented with $100 \mu \mathrm{g} \mathrm{mL}^{-1}$ of ampicillin or $50 \mu \mathrm{gL}^{-1}$ of kanamycin when needed.

\section{Fluorescent dye delivery}

Microalgal cells in the exponential phase $\left(5 \times 10^{6}-1 \times 10^{7}\right.$ cells $\left.\mathrm{ml}^{-1}\right)$ were collected by centrifugation at $2500 \mathrm{xg}$ for $10 \mathrm{~min}$ at $4^{\circ} \mathrm{C}$. The pellet was washed with electroporation buffer $(5.0 \mathrm{mM} \mathrm{KCl}, 5.0 \mathrm{mM} \mathrm{CaCl}$, $0.01 \mathrm{M}$ HEPES, $0.1 \mathrm{M}$ mannitol and $0.1 \mathrm{M}$ sorbitol), and resuspended to a final concentration of $10^{8}$ cells $\mathrm{ml}^{-1}$ in cold electroporation buffer. From this cell suspension $250 \mu \mathrm{L}$ was transferred to a 2-mm electroporation cuvette. Sytox Green (Life Technologies, Grand Island, NY, USA; S7020) was added to a final concentration of $1 \mu \mathrm{M}$ and incubated in the dark for 5 minutes on ice. This dye was used to assess the level of permeability as a result of the electric pulse. Electroporation was carried out using a Bio-Rad Gene-Pulser apparatus set at $25 \mu \mathrm{F}$ and infinite resistance and varying yield strengths $\left(0-10.5 \mathrm{kV} \mathrm{cm}^{-1}\right)$. After electroporation, the cell suspension was recovered in the dark for 5 minutes on ice. $1 \mathrm{~mL}$ of electroporation buffer was added and the cells were incubated for 10 minutes in the dark at room temperature. Cells that were assessed for cell viability were 
not exposed to Sytox Green but were supplemented with propidium iodide (Life Technologies, Inc., Carlsbad, CA) at a final concentration of $60 \mu \mathrm{M}$, after the recovery phase, before analysis by fluorescent microscopy or flow cytometry.

\section{Labelled DNA and labelled protein delivery}

The plasmid pOpt-Clover-Hyg (Lauersen et al., 2015) was extracted from $E$. coli DH5a (Chlamydomonas Resource Center) using the Gene Jet Plasmid Miniprep kit (Thermo Scientific). $5 \mu \mathrm{g}$ of purified plasmid was labelled at a $1: 1$ $(v: w)$ ratio of Label IT® Fluorescein to nucleic acid according to manufacturer instructions (Mirus, Madison, WI, USA). Fluorescein isothiocyanate labelled bovine serum albumin (FITC-BSA) was delivered into the cells at a final concentration of $10 \mu \mathrm{M}$ (Thermo Fisher). Labelled DNA and labelled BSA were delivered into Chlamydomonas reinhardtii, Acutodesmus obliquus, Chlorella vulgaris and Neochloris oleoabundans with electroporation conditions mentioned above and cellular uptake was analysed by fluorescent microscopy or flow cytometry.

\section{Fluorescence screening}

Cells were visually inspected with an Olympus IX71S8F-3 fluorescence microscope with 460-495 nm excitation filter, 510-550 nm Emission filter and a 505-nm dichromatic mirror for Sytox Green. High throughput quantification of the dye uptake was done by using a BD Accuri C6 flow cytometry (BD Biosciences, San Jose, CA, USA). The electroporated cells were diluted 1:200 in electroporation buffer, before flow cytometry analysis to obtain an optimal flow rate for the detectors. Per condition 20.000 cells were measured with an argon-ion excitation laser $(488 \mathrm{~nm})$ and detected using three different fluorescent channels: green (FL1 $530 \pm 15 \mathrm{~nm}$ ) for Sytox Green, labelled DNA and labelled BSA, orange (FL2 $585 \pm 20 \mathrm{~nm}$ ) for propidium iodide, and red (FL3 $670 \pm 25 \mathrm{~nm}$ ) for chlorophyll autofluorescence using a flowrate of $14 \mu \mathrm{L} \mathrm{min}{ }^{-1}$. BD Accuri C6 Software 264.21 was used for data acquisition and analysis. Forward scatter and side scatter plots were used to remove background noise and cellular debris. Sytox Green uptake was analysed by plotting log autofluorescence (FL3) versus log green fluorescence (FL1), to discriminate 
between cells that did and did not take up Sytox Green, labelled DNA and labelled BSA. Propidium iodide uptake was analysed by plotting log autofluorescence (FL3) versus log orange fluorescence (FL2).

\section{Microalgal transformation}

Chlamydomonas reinhardtii, Acutodesmus obliquus, Chlorella vulgaris and Neochloris oleoabundans cells were harvested, washed, and re-suspended as mentioned before. Linearized plasmid pOpt-Clover-Hyg at a final concentration of $2 \mu \mathrm{g} \mathrm{mL}^{-1}$ and $25 \mu \mathrm{g} \mathrm{mL}^{-1}$ of boiled salmon sperm DNA (D1626, Sigma) were added to the cell suspension and kept on ice for 15 minutes. From this transformation mixture $250 \mu \mathrm{L}$ was transferred into a 2-mm electroporation cuvette, followed by electroporation at $2.25,5,6$ and $7.5 \mathrm{kV} \mathrm{cm}^{-1}$ respectively. The cells were recovered in the dark for 10 minutes, then transferred into a sterile $15 \mathrm{~mL}$ tube containing $10 \mathrm{~mL}$ of freshwater medium and kept in the dark overnight at $25^{\circ} \mathrm{C}$ with continuous agitation at $125 \mathrm{rpm}$. After recovery, cells were collected by centrifugation at $2500 \mathrm{xg}$ for 10 minutes at room temperature. The cell pellet was re-suspended in $200 \mu \mathrm{L}$ cultivation medium and $1 \times 10^{8}$ cells were plated onto selective agar plates with the selected hygromycin B concentrations. The antibiotic concentrations were determined for each microalgae species using an antibiotic sensitivity test. All selective plates were incubated under a day:night cycle regime $(16: 8)$ at $25^{\circ} \mathrm{C}$ at $60 \mu \mathrm{mol} \mathrm{m} \mathrm{m}^{-2} \mathrm{~s}^{-1}$ light intensity (Grolux fluorescent tubes, Sylvania F36W/GRO) at $125 \mathrm{rpm}$ and $2.5 \% \mathrm{CO}_{2}$.

\section{Antibiotic sensitivity test}

The sensitivity of all microalgae species to hygromycin B was tested. An initial concentration of $1 \times 10^{8}$ cells were grown on TAP and FW agar plates supplemented with hygromycin $\mathrm{B}$. The antibiotic was added to the medium in a concentration range from 0 to $100 \mu \mathrm{gL}^{-1}$. The test was performed for 20 days and the minimal concentration at which the growth of each specie was completely inhibited, was used for the selection of transformants. 


\section{Transformation of $A$, obliquus}

Acutodesmus obliquus was harvested, washed, and re-suspended as mentioned before. Some modifications in the procedure were made to achieve successful transformation and further improvements in transformation efficiency. Linearized plasmid pBBR1-GFP-Hyg or PCR amplified cassette at a final concentration of $2 \mu \mathrm{g} \mathrm{mL}^{-1}$ and $25 \mu \mathrm{g} \mathrm{mL}^{-1}$ of boiled salmon sperm DNA were added to the cell suspension and kept on ice. The transformation mixture was transferred into a 2-mm electroporation cuvette and followed by electroporation at $7 \mathrm{kV} \mathrm{cm}^{-1}$. The cells were recovered overnight in freshwater medium as previously mentioned. After recovery, cells were collected, resuspended and both $5 \times 10^{7}$ and $1 \times 10^{8}$ cells were plated onto selective agar plates with the selected hygromycin $\mathrm{B}$ concentration. All selective plates were incubated under day:night cycle regime $(16: 8)$ at $25^{\circ} \mathrm{C}$ at 25 and $60 \mu \mathrm{mol} \mathrm{m}{ }^{-2}$ $\mathrm{s}^{-1}$ light intensity at $125 \mathrm{rpm}$ and $2.5 \% \mathrm{CO}_{2}$.

\section{Plasmid construction}

Two cassettes containing either the GFP*His6 or aph7 gene under the control of the CaMV35S promoter and terminator were codon optimized according to the codon usage of $A$. obliquus (Eugene v1.4.0 software), synthesized (Integrated DNA Technologies, Inc., USA) and assembled into plasmid pBBR1MCS2 (Addgene) previously linearized with KpnI restriction enzyme (Thermo Fisher), resulting in plasmid pBBR1-GFP-Hyg. Plasmid assembly was performed using HiFi DNA Assembly Master Mix (NEB) according to the manufacturer's recommended protocol and transformed into $E$. coli S17 competent cells. The competent cells were prepared by using the Mix \& Go $E$. coli Transformation Kit (Zymo research).

In order to transform A. obliquus, the plasmid pBBR1-GFP-Hyg was extracted using the Gene Jet Plasmid Miniprep kit (Thermo Scientific) and either linearized plasmid or the expression cassettes previously PCR amplified were transformed into the algae. 
The plasmid pBBR1-GFP-Hyg was digested by KpnI restriction enzyme (Thermo Scientific) and the region containing both expression cassettes including GFP*His6 and aph7 genes were PCR amplified. The primers used were F-pBBR-

In: $\quad 5^{\prime}$-GACTCACTATAGGGCGAATTG- $3^{\prime}$ and R-pBBR-In: 5'CACACAGGAAACAGCTATGA-3'. The PCR conditions were as follows: initial denaturation at $98^{\circ} \mathrm{C}$ for $1 \mathrm{~min}$, denaturation at $98^{\circ} \mathrm{C}$ for $10 \mathrm{~s}$, annealing at 65 ${ }^{\circ} \mathrm{C}$ for $30 \mathrm{~s}$ and extension at $72{ }^{\circ} \mathrm{C}$ for $3 \mathrm{~min}$, for 35 cycles, followed by a final extension at $72{ }^{\circ} \mathrm{C}$ for 2 min, with Q5 Hot Start High-Fidelity DNA Polymerase (NEB).

\section{Microalgal cell disruption and colony PCR}

Microalgal cells were collected from selective agar plates and transferred into a Lysing Matrix Tube D (MP Biomedicals) containing $300 \mu \mathrm{l}$ of $0.2 \%$ SDS. Samples were homogenized twice for 60 seconds at speed of $4000 \mathrm{rpm}$ with a BeadBeater (Precellys 24). The lysis was followed by a phase separation with Phenol:Chloroform:Isoamyl alcohol (25:24:1), DNA precipitation with ethanol and resuspended in TE buffer. Selection of positive transformants was performed by PCR amplification of the hygromycin resistance gene (Aph7). The primers used were F-aph7: 5' - AGTTCCTCATCGAGAAGTTCGACAG - 3' and Raph7: 5' - CCAGAAGAAGATGTTTGCCACCTC - 3'. The PCR conditions were as follows: initial denaturation at $95^{\circ} \mathrm{C}$ for $2 \mathrm{~min}$, denaturation at $95^{\circ} \mathrm{C}$ for $30 \mathrm{~s}$, annealing at $55^{\circ} \mathrm{C}$ for $30 \mathrm{~s}$ and extension at $72{ }^{\circ} \mathrm{C}$ for $1 \mathrm{~min}$, for 35 cycles, followed by a final extension at $72{ }^{\circ} \mathrm{C}$ for $10 \mathrm{~min}$, with DreamTaq DNA Polymerase (Thermo Fisher).

\section{Statistical analysis}

All the experiments in this study were performed in biological triplicates. The data were represented as mean \pm standard deviation (SD). 


\section{RESULTS}

\section{Fluorescent dye delivery}

The development of a method to deliver molecules into microalgae cells can be complicated and time-consuming. Therefore, we set out to test a fluorescent dye-based method to assess electroporation settings that can lead to a successful transformation. We developed a screening method to test the degree of permeabilization of the cell membrane and its influence on viability after an electric pulse is applied. We used two different dyes that are nonpermeant to intact cells: Sytox Green (SG) and propidium iodide (PI), and we tested them in four microalgal species using the experimental design described in figure 1. Cells of the four microalgal species were electroporated in the presence of SG and, after recovery, the permeable population was determined by flow cytometry. A second portion of the cells was electroporated and PI was

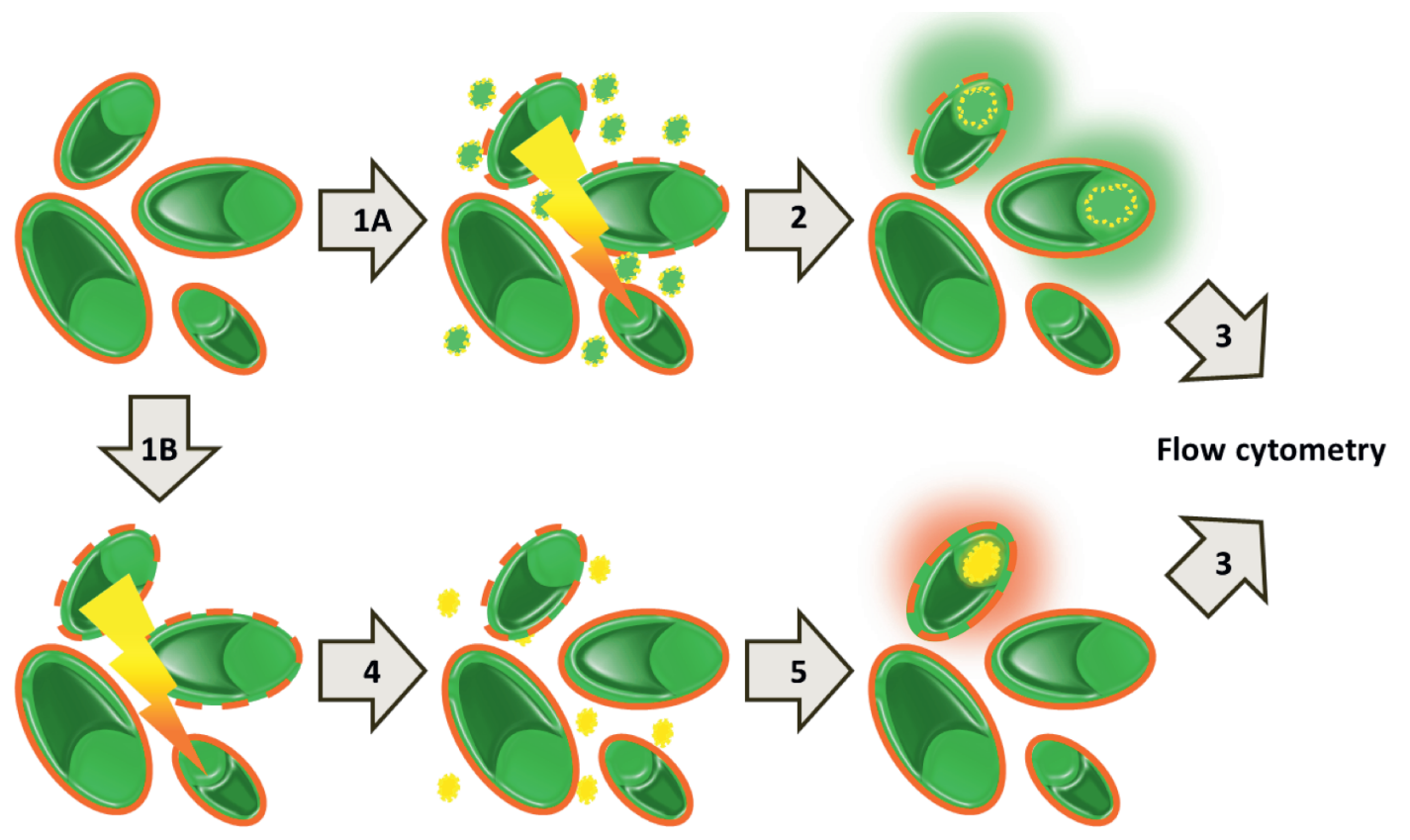

Figure 1. Experimental design for cell competence screening. (1A) Electroporation in the presence of Sytox Green. (2) Recovery phase. (3) Fluorescence uptake monitored with flow cytometer. (1B) Electroporation without fluorescent dye. (4) At the end of the recovery propidium iodide is added. (5) Propidium iodide enters non-viable cells. Orange line represents the cell membrane and cell wall. 
added after the recovery phase to determine the cell population that did not close the pores induced by the electric pulse. These cells are considered to be non-viable (figure 1).

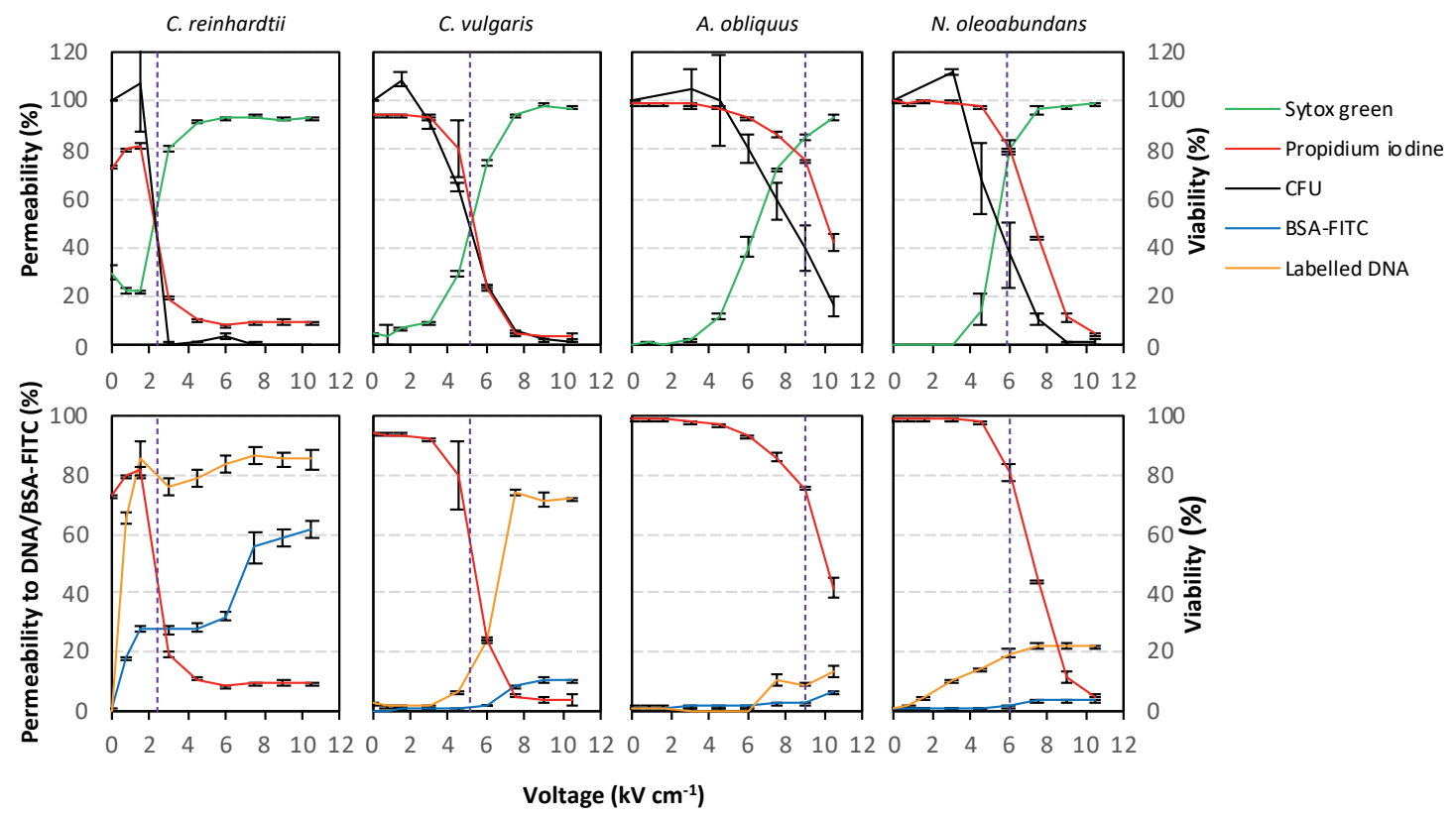

Figure 2. Curves showing response to different voltage setting for the four tested microalgal species. C. reinhardtii, C. vulgaris and $A$. obliquus are tested. Curves represent the percentage of total cells that become permeable to Sytox Green, labelled DNA and BSA-FITC (Left Y-axis). Uptake of propidium iodide is a proxy for cell viability after an electric pulse is applied (Right $\mathrm{Y}$-axis). The black line shows the colony forming units (CFU) after plating the electroporated cells (Right Y-axis). Dashed line shows proposed voltage setting. The data represent the average of $n=3$ replicate experiments. Standard deviation bars are shown.

In figure 2, viability and permeability of four microalgae species are shown for different voltage settings (For examples of flow cytometer output see Supplementary figure 1). Although the difference in response of permeability and viability differs from species to species, all species show a similar pattern. The higher the voltage that was applied the higher the SG uptake was, representing the permeabilized cell population. The viability measured by PI uptake decreased when a higher voltage was applied. An intersection between the two curves was observed, however the voltage corresponding to this specific moment differs for all species. C. reinhardtii is the most prone to take 
up SG and the intersection of the viability and permeability curves lies around $2.25 \mathrm{kV} \mathrm{cm}^{-1}$. C. vulgaris has a slightly higher resistance around $5 \mathrm{kV} \mathrm{cm}^{-1}$. The oleaginous microalgae $A$. obliquus and $N$. oleoabundans seem to be even more resistant and the curves intersect at around 8.25 and $6 \mathrm{kV} \mathrm{cm}^{-1}$ respectively.

The viability measured by PI uptake was supported by colony forming unit (CFU) values obtained by plating electroporated cells (figure 2). Most of the species show an increase in viability in the CFU count after electroporation in the low range $\left(0.75-3 \mathrm{kV} \mathrm{cm}^{-1}\right)$. Although the values obtained with PI and CFU follow a similar pattern, some nonviable cells may not have taken up PI resulting in a slight overestimation of the viable cell population. The balance between viability and permeability makes electroporation a platform that needs optimization. We defined competent cells as the cells that form micro-pores, are able to close these pores and are viable thereafter. In figure $\mathbf{2}$ a voltage setting was assigned for all strains to represent the competent cell fraction, which lies around the intersection of the permeability and viability curves. The proposed voltage settings for every species are compared with the values described in literature in the table $\mathbf{1}$.

Table 1. Proposed voltage settings for the tested microalgae in this study compared to voltage settings described in literature.

\begin{tabular}{|c|c|c|c|}
\hline $\begin{array}{l}\text { Microalgal } \\
\text { species }\end{array}$ & $\begin{array}{l}\text { Proposed voltage } \\
\qquad\left(\mathrm{kV} \mathrm{cm}^{-1}\right)\end{array}$ & $\begin{array}{l}\text { Voltage used in literature } \\
\qquad\left(\mathrm{kV} \mathrm{cm}^{-1}\right)\end{array}$ & Ref. \\
\hline C. reinhardtii & 2.25 & 2 & $\begin{array}{c}\text { (Rasala et al., } \\
\text { 2012) }\end{array}$ \\
\hline C. vulgaris & 5 & 5 & (Koo et al., 2013) \\
\hline A. obliquus & 8.25 & $10 *$ & $\begin{array}{l}\text { (Guo et al., } \\
\text { 2013)* }\end{array}$ \\
\hline N. oleoabundans & 6 & n.d. & - \\
\hline
\end{tabular}




\section{Labelled DNA and labelled protein delivery}

The fluorophores used in this study are considerably smaller than proteins and nucleic acid molecules. We therefore analysed uptake of labelled DNA and labelled proteins and compared them with permeability results obtained with fluorophores. We used an average size plasmid $(6.8 \mathrm{~Kb})$ pOpt-Clover-Hyg (linearized and labelled with fluorescein) and the $66 \mathrm{kDa}$ bovine serum albumin protein labelled with fluorescein isothiocyanate (FITC-BSA). These fluorescent molecules were detected and analysed by flow cytometry with the FL1 fluorescent channel after the electric pulse was applied.

We performed permeability experiments in the microalgae Chlamydomonas reinhardtii, Acutodesmus obliquus, Chlorella vulgaris and Neochloris oleoabundans. Cells were electroporated with different voltages following the protocol described in figure 1. Labelled DNA or labelled proteins instead of SG were added to the cell suspension. In figure $\mathbf{2}$, the uptake of labelled DNA and labelled proteins is shown for different voltage settings. As shown in SG uptake results, the higher the voltage applied the higher the uptake of labelled DNA and protein was. Our results indicate that bigger molecules are less prone to be taken up by the cells even when high electric pulses were applied. Moreover, the microalgal cells seem to be more permeable to DNA than proteins. At high voltages, $C$. reinhardtii and $C$. vulgaris are the most prone to take up these molecules. Around $84 \%$ and $74 \%$ of the cells were able to take up labelled DNA, whereas $60 \%$ and $10 \%$ of the cells took up BSA-FITC, respectively. $N$. oleoabundans and $A$. obliquus were less permeable, $21 \%$ and $13 \%$ of the cells took up labelled DNA, while only $4 \%$ and $6 \%$ of the cells took up BSA-FITC, respectively. However, at high voltages the cell viability was considerably lower. When using the proposed voltage settings lower cell permeability to DNA and proteins is obtained, however significantly higher cell viability was observed which increases delivery efficiencies. The percentage of permeable cells to DNA and proteins when the proposed voltage settings were applied is shown in table 2. 
Table 2. Permeable cells to labelled DNA and labelled protein at proposed voltage settings.

\begin{tabular}{lccc}
\hline $\begin{array}{l}\text { Microalgal } \\
\text { species }\end{array}$ & $\begin{array}{c}\text { Proposed voltage } \\
\left(\mathrm{kV} \mathrm{cm}^{-1}\right)\end{array}$ & $\begin{array}{c}\text { Cells permeable to } \\
\text { DNA } \\
(\%)\end{array}$ & $\begin{array}{c}\text { Cells permeable } \\
\text { to BSA } \\
(\%)\end{array}$ \\
\hline C. reinhardtii & 2.25 & 80 & 19.0 \\
C. vulgaris & 5.0 & 15 & 1.3 \\
A. obliquus & 8.25 & 9 & 2.7 \\
N. oleoabundans & 6 & 20 & 1.7 \\
\hline
\end{tabular}

\section{Microalgal transformation via electroporation}

We performed the genetic transformation of Chlamydomonas reinhardtii to verify that our predicted voltage settings lead to a high transformation efficiency. We used the pOpt-Clover-Hyg vector which was specifically designed for the transformation of this alga. It was delivered into the cells using the proposed voltages described in table 1 . We obtained colonies on selective plates containing $10 \mu \mathrm{g} \mathrm{mL}^{-1}$ of hygromycin B after 2-3 weeks of incubation. These were confirmed by PCR amplification of the antibiotic resistance gene aph7. Out of 350 colonies visible on selective plates 190 were positive transformants which corresponds to a transformation efficiency of 76 transformants per $\mu \mathrm{g}$ of DNA.

No positive transformants were obtained for Acutodesmus obliquus, Chlorella vulgaris and Neochloris oleoabundans on selective plates containing 50, 50 and $10 \mu \mathrm{g} \mathrm{mL} \mathrm{m}^{-1}$ of hygromycin B respectively. All species were transformed with pOpt-Clover-Hyg vector, applying the proposed conditions described in table 1. This shows that it is indeed difficult to use transformation efficiency to optimize electroporation settings.

We further optimized conditions involved after the electroporation step in $A$. obliquus, which has shown to be one of the most resistant species to take up 
extracellular molecules such as DNA and proteins. We tested a functional expression plasmid, different DNA fragment sizes and factors that could have an influence on the appearance of false-positives such as light intensity and cell concentration.

A. obliquus was transformed with DNA fragment sizes of $8.5 \mathrm{~Kb}$ (linearized plasmid pOpt-Clover-Hyg), 6.8Kb (linearized plasmid pBBR1-GFP-HYG) and $3.5 \mathrm{~Kb}$ (the expression cassette PCR amplified from pBBR1-GFP-HYG). Electroporation was carried out with a voltage of $9 \mathrm{kV} \mathrm{cm}^{-1}$ and electroporated cells were grown on selective plates and exposed to two light intensities: 25 and $60 \mu \mathrm{mol} \mathrm{m} \mathrm{m}^{-2} \mathrm{~s}^{-1}$.

As shown in figure $\mathbf{2}, 9 \%$ of $A$. obliquus cells were permeable to the labelled plasmid pOpt-Clover-Hyg and $75 \%$ of the cells were viable when $9 \mathrm{kV} \mathrm{cm}^{-1}$ was applied. However, as shown in figure $\mathbf{3 b}$, no transformants were obtained. Although 19 and 280 colonies were visible on plates when exposed to either 25 or $60 \mu \mathrm{mol} \mathrm{m} \mathrm{m}^{-2} \mathrm{~s}^{-1}$ of light intensity respectively, these were shown to be false positives by PCR. It is likely that DNA was taken up, however no viable transformants were obtained. These results indicate that further optimization is required to achieve the successful transformation of $A$. obliquus. Consequently, the expression cassette containing both the aph7 gene conferring resistance to hygromycin B and the green fluorescent protein (GFP*His6) under the promoter and terminator CaMV35S was codon optimized, synthesized and assembled into pBBR1-GFP-HYG (figure 3a). As shown in figure $\mathbf{3 b}$, positive transformants were obtained when linearized plasmid pBBR1-GFP-HYG or expression cassettes PCR amplified from plasmid pBBR1GFP-HYG were delivered into the cells. The highest transformation efficiency of 125 transformants per $\mu \mathrm{g}$ of DNA was achieved when a small fragment was used and transformants were exposed to a low light intensity (figure $\mathbf{3 b} \mathbf{b}-\mathbf{c}$ ). Delivery of a PCR product which is 2.4 times smaller than the linearized plasmid yielded a 2.6-fold increase in the transformation efficiency when the same conditions were applied. 
Further optimization experiments were performed by varying the number of cells plated on selective agar plates after electroporation. $5 \times 10^{7}$ and $1 \times 10^{8}$ cells were plated onto agar plates containing hygromycin B. Genetic transformation of $A$. obliquus was performed with proposed voltage conditions showed in figure 2. An electric pulse of $9 \mathrm{kV} \mathrm{cm}^{-1}, 25 \mu \mathrm{mol} \mathrm{m}^{-2} \mathrm{~s}^{-1}$ of light intensity and PCR amplified fragment were used. A higher transformation efficiency of 108 transformants per $\mu \mathrm{g}$ of DNA was achieved when $5 \times 10^{7}$ cells $\mathrm{mL}^{-1}$ were used. Although, fewer colonies were obtained on plates, only 10 were false-positives from a total of 280 colonies. On control plates where no plasmid was added to the electroporation mixture, colonies were still able to grow (data not shown).
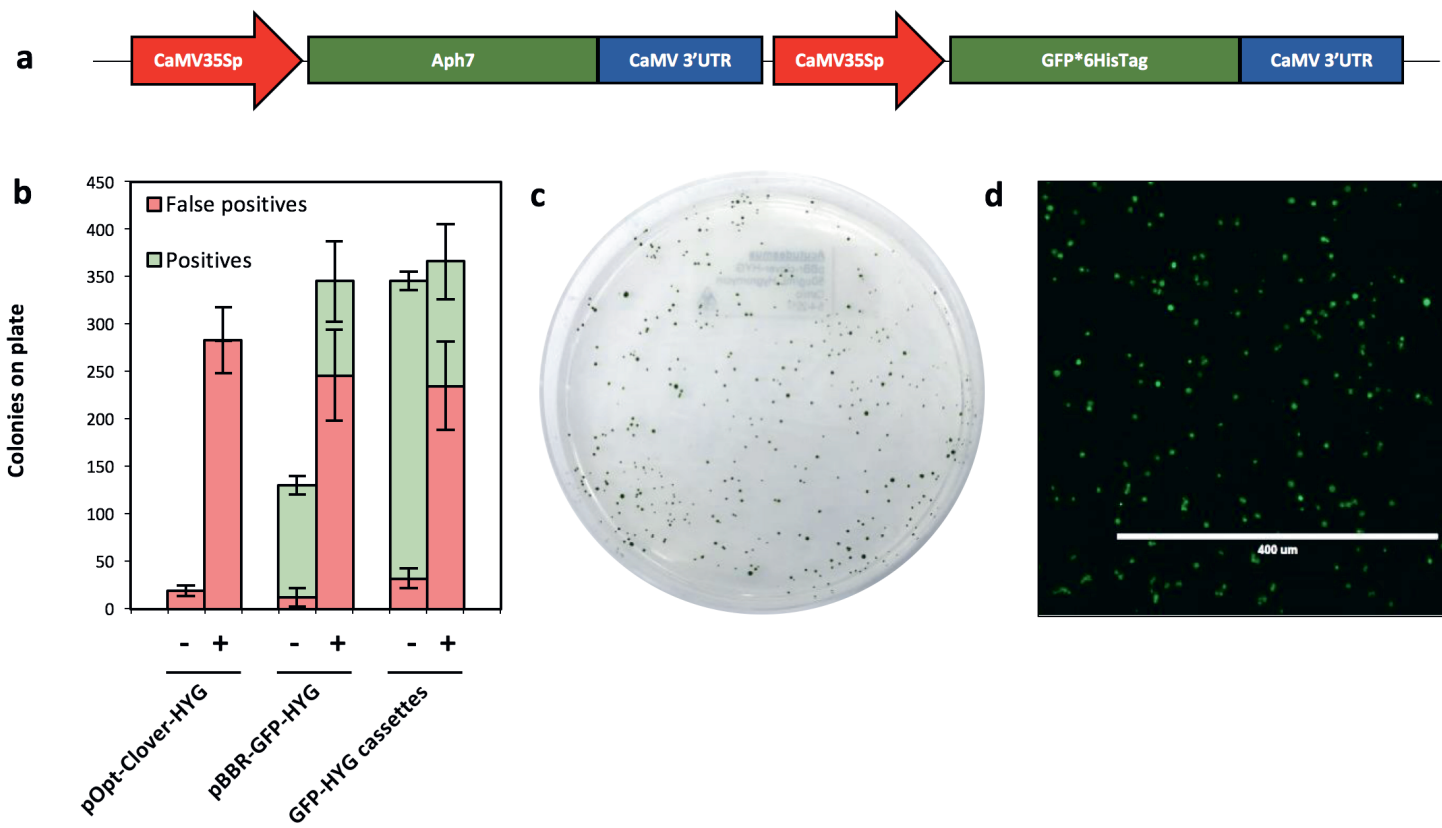

d

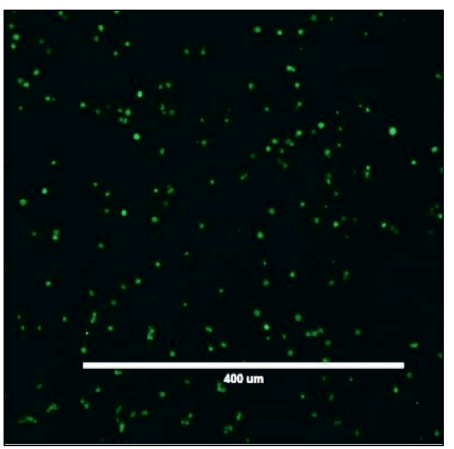

Figure 3. Transformation of Acutodesmus obliquus. (a) Schematic structure of the expression cassette contained in the plasmid pBBR1-GFP-HYG. (b) Number of positive (green bars) and false-positive (red bars) transformants obtained on selective plates when an electric pulse of $9 \mathrm{kV} \mathrm{cm}^{-1}$ is applied. Plates were exposed to $25(-)$ and $60(+)$ $\mu \mathrm{mol} \mathrm{m} \mathrm{m}^{-2} \mathrm{~s}^{-1}$ of light intensity. Three fragments were delivered into the cells: linearized plasmid pOpt-Clover-Hyg, linearized plasmid pBBR1-GFP-HYG and the expression cassette PCR amplified from pBBR1-GFP-HYG. (c) Colonies obtained on freshwater agar plates containing hygromycin B. Transformation was performed with PCR fragment and selection plate was exposed to a light intensity of $25 \mu \mathrm{mol} \mathrm{m} \mathrm{m}^{-2} \mathrm{~s}^{-1}$. (d) Fluorescence microscope image of GFP expressed in $A$. obliquus cells. The data represent the average of $n=3$ replicate experiments. Standard deviation bars are shown. 
High cell concentration leads to appearance of false positives either as lawn or patches of small colonies, which is probably caused by a reduced contact with the antibiotic. Therefore, our results suggest that a lower cell concentration, low light intensity and delivery of a small DNA fragment yield a higher transformation efficiency.

\section{Discussion}

Transformation platforms for $C$. reinhardtii are well established and a wide range of techniques are used to metabolically engineer this model species. For other promising microalgae species, genetic engineering is however hindered due to the absence of efficient transformation methods. Successful transformation of microalgae relies not only on the delivery technique used but also on whether the delivered expression vector is functional. Therefore, efficient delivery cannot be assessed by testing transformability and a higher transformation efficiency cannot be reached by determining optimal delivery settings of the technique tested. Consequently, a method to verify that extracellular molecules are effectively delivered into the cells could tackle this issue.

In our study, a straightforward and effective electroporation optimization tool for microalgal species is proposed. Cell competence can be monitored by measuring cell permeability and viability by using Sytox Green and propidium iodide respectively. Both fluorophores were chosen because of their properties: they are not permeant to living cells and intercalate between the bases of nuclear DNA which intensifies the fluorescence by more than 500-fold and 2030-fold, respectively.

Initially we aimed to develop a one-step screening method that would show the level of permeability and viability of the tested settings simultaneously. However, the emission spectra of both intercalating fluorophores overlapped in the flow cytometer analysis, and the fluorescence level of SG was much higher than PI. Although, FL3 shows the PI fluorescence and less of the SG, the autofluorescence of chlorophyll masks the measurements. For this reason, we 
decided to develop a two-step screening method where permeability and viability effects are measured separately after an electric pulse is applied.

All species tested showed a similar pattern. The higher the voltage applied, the higher the permeabilized population and the lower the viability was. The viability values determined by CFU counting were slightly higher than results obtained with PI at low voltages. This could be the result from dissociation of small aggregates of microalgal cells that resulted from the washing steps prior to the electroporation. The flow cytometer will find more small events when the cells are dissociated by electroporation and larger aggregates when no electric pulse is applied. Furthermore, we observed an intersection between the permeability and viability curves. The voltages corresponding to these intersection points were selected and proposed for the transformation of each microalgae species. The proposed values for every species correspond well with the values described in literature which is a good indication for the validity of the presented method (table 1).

Additionally, we tested the proposed method in the marine specie Nannochloropsis $s p$. However, this microalga showed different curves with a reduced viability prior to electroporation. We used a low salt electroporation buffer to enable high voltage settings causing an osmotic shock that permeabilized around $32 \%$ of the cells (data not shown). If the salinity of the buffer would be increased, the osmotic shock and high death rate would be reduced, but electric arcing at higher voltages are likely to occur and therefore the use of low salt buffers was required. The electroporation buffers described in literature for Nannochloropsis $s p$. are similar to the one we tested (Radakovits et al., 2012). Due to the high mortality rate, we were not able to determine a voltage setting that could lead to the successful transformation of Nannochloropsis sp.

In our results, we observed that permeability and viability differ from species to species. We hypothesize that these differences are caused by the structure and composition of the microalgal cell wall. The cell wall of $C$. reinhardtii lacks cellulose and is composed of hydroxyproline-rich glycoproteins (Macfie et al., 
1994; Voigt, 1988). A. obliquus has a more complex cell wall which consist of an inner cellulosic layer surrounded by a trilaminar structure known as algaenan. The trilaminar algaenan structure is still unknown, but it has been characterized for its physical and chemical properties (Allard et al., 1998). Layers 1 and 3 of the trilaminar structure are suggested to contain glycoproteins and glucosamine containing biopolymers (Jan Burczyk et al., 1999). The middle layer is hydrophobic which suggests the presence of lipids (J. Burczyk et al., 1970). C. vulgaris has a cell wall containing glucosamine, cellulose and other polysaccharides containing mainly rhamnose and arabinose (Pieper et al., 2012). The cell wall of $N$. oleoabundans contains cellulose (Chantanachat, 1962; D. Wang et al., 2015), however it has not been studied in much detail and there are no reports describing the presence of an algaenan cell wall. Based on cell wall composition of the tested species we would expect that $C$. reinhardtii and $C$. vulgaris are the most susceptible species to be permeabilized followed by $N$. oleoabundans and $A$. obliquus which have more rigid cell walls.

As expected, higher permeability/viability values were obtained when using SG and PI. The micro-pores formed in the cell wall after the electrical pulse is applied might have a restrictive size, conferring a more accessible passage for small molecules.

Therefore, the results obtained with fluorophores must be taken as a proxy for the permeabilization of the cell wall and cell membrane since they do not directly predict the efficiency of the uptake of larger molecules. Consequently, we tested bigger molecules such as FITC-BSA and an average size plasmid previously labelled with fluorescein. Since fluorescein is a non-enzyme mediated fluorophore, the labelling efficiency of the reaction is not dictated by the enzymatic ability to incorporate labelled nucleotides. Fluorescein binds covalently to DNA and has a labelling efficiency of approximately one label every 20-60 base pairs of double stranded DNA, which was sufficient to allow detection by flow cytometry. 
Our results indicate that larger molecules are less prone to be taken up by the cells. High voltage ranges seem to show the most favourable permeability results. However, it is not recommended to use such high voltages since viability decreases significantly and therefore, to increase delivery efficiencies, lower voltages have to be used. Although a small portion of cells were permeable to proteins and DNA, the cell permeability seems to follow the predicted pattern obtained in SG uptake experiments. We recommend using the proposed voltage settings obtained with SG and PI to ensure successful delivery of DNA and proteins without affecting considerably the cell viability. Our results suggest that slightly higher voltage setting can be used to increase uptake, although cell viability has to be taken into consideration.

We demonstrated that a high transformation efficiency can be accomplished when predicted values are used, as shown in $C$. reinhardtii and $A$. obliquus. Furthermore, our results are in accordance with values found in literature for DNA transformation of the model species $C$. reinhardtii and $C$. vulgaris and the oleaginous species $A$. obliquus (strain FSP-3). Therefore, the method is a good indicator whether the conditions tested are suitable for actual DNA transformation. However, we also revealed that further optimization is needed. Appropriate promoters, terminators, optimal codon usage and other factors involved in the cultivation of transformants can increase the transformation efficiency. Our experiments demonstrate that low cell concentrations, low light intensities and delivery of small DNA fragments yield higher transformation efficiencies. We suspect that high light intensities could reduce functionality of the antibiotic and a high cell concentration could reduce the contact of the cells with the selective plates resulting in false-positives.

The construction of functional expression vectors is another key element for successful transformation of microalgae. In our first attempts, in order to increase the probability of success we used an average size and non-replicative plasmid pOpt-Clover-Hyg which contains commonly used genetic elements for the transformation of microalgae such as Aph7 gene which confers hygromycin $B$ resistance and clover green fluorescent protein. Both genes were driven by the ribulose-1,5-bisphosphate carboxylase small subunit 2 promoter (RBCS2) 
linked to a heat shock protein 70A promoter (HSP70Ap). However, since pOpClover-Hyg plasmid has been designed for Chlamydomonas, no positive transformants were obtained when tested in other algae species.

We achieved the successful transformation of $A$. obliquus when we used the heterologous promoter and terminator derived from the Cauliflower Mosaic Virus to drive the expression of the codon optimized Aph7 and GFP genes. However, endogenous regulatory sequences could significantly increase gene expression (Vila et al., 2012). Other regulatory elements such as the enhancer HSP70A, RBCS2 introns and inclusion of splicing signals could lead to higher expression levels (Doron et al., 2016). Moreover, exogenous genes may contain codons that are rarely used or expression-limiting regulatory elements which could affect the expression, structure and function of the encoded proteins in the expression host (Heitzer et al., 2007). Thus, optimization or harmonization of the codon usage might be necessary for correct gene expression and protein folding.

Our method can be used to optimize parameters in electroporation protocols, such as voltage, resistance, cuvette size and osmotic buffer. Optimization of other transformation tools such as glass beads and polyethylene glycol mediated transformation could benefit from this method as well. The electroporation optimization tool proposed in this study can be used for purposes other than DNA delivery. The development of genome editing techniques are becoming essential to investigate gene functions and improve traits by suppression or reduction of expression levels. Currently, CRISPR-Cas9 and CRISPR-Cpf1 technologies can be used to generate knockout/knock-in mutants (S. Jeon et al., 2017). The proteins Cas9 or Cpf1 can be preassembled with guide RNA in vitro and delivered as ribonucleoproteins by electroporation into the cells. Knockdown mutants can also be created by delivery of small interfering RNAs (siRNA) or microRNAs (miRNAs) via electroporation (Baek et al., 2016; S. Jeon et al., 2017). We suggest to use the proposed protocol to measure cell permeability to ribonucleoproteins and RNAi previously labelled with suitable fluorophores. Using this method, delivery of extracellular molecules in the target species can now be studied and optimized, thereby the 
bottlenecks of nucleic acids/proteins delivery can be ruled out when transformation remains unsuccessful.

\section{Acknowledgements}

This research project is financially supported by the Food and Nutrition Delta program of Agentschap NL (FND10007), Unilever and the National Commission of Scientific and Technologic Research of Chile (CONICYT).

\section{Author's contribution}

CFM, $\mathrm{L}$ and MHJS designed the experiment, analysed and interpreted the data. CFM and $\sqcup J$ wrote the manuscript. CFM, L, MHJS, KYFL, JWJO and EJHW performed the experiments. MHJS, JS, DEM, GE, RAW and RHW supervised the project. All authors contributed to the work, discussed the results, read and approved the final version of this manuscript.

\section{Conflict of interest statement}

We declare that there are no conflicts of interest related to this work.

\section{Statement of informed consent, human/animal rights}

No conflicts, informed consent, human or animal rights applicable 


\section{Supplementary information}

Supplementary figure 1. Flow cytometer output for permeability (a) and viability (b). Sytox green uptake analysed by plotting log chlorophyll autofluorescence (FL3 $670 \pm 25$ $\mathrm{nm}$ ) versus log green fluorescence (FL1 $530 \pm 15 \mathrm{~nm}$ ). Propidium iodide uptake analysed by plotting log chlorophyll autofluorescence (FL3) versus log orange fluorescence (FL2 $585 \pm 20 \mathrm{~nm}$ ).

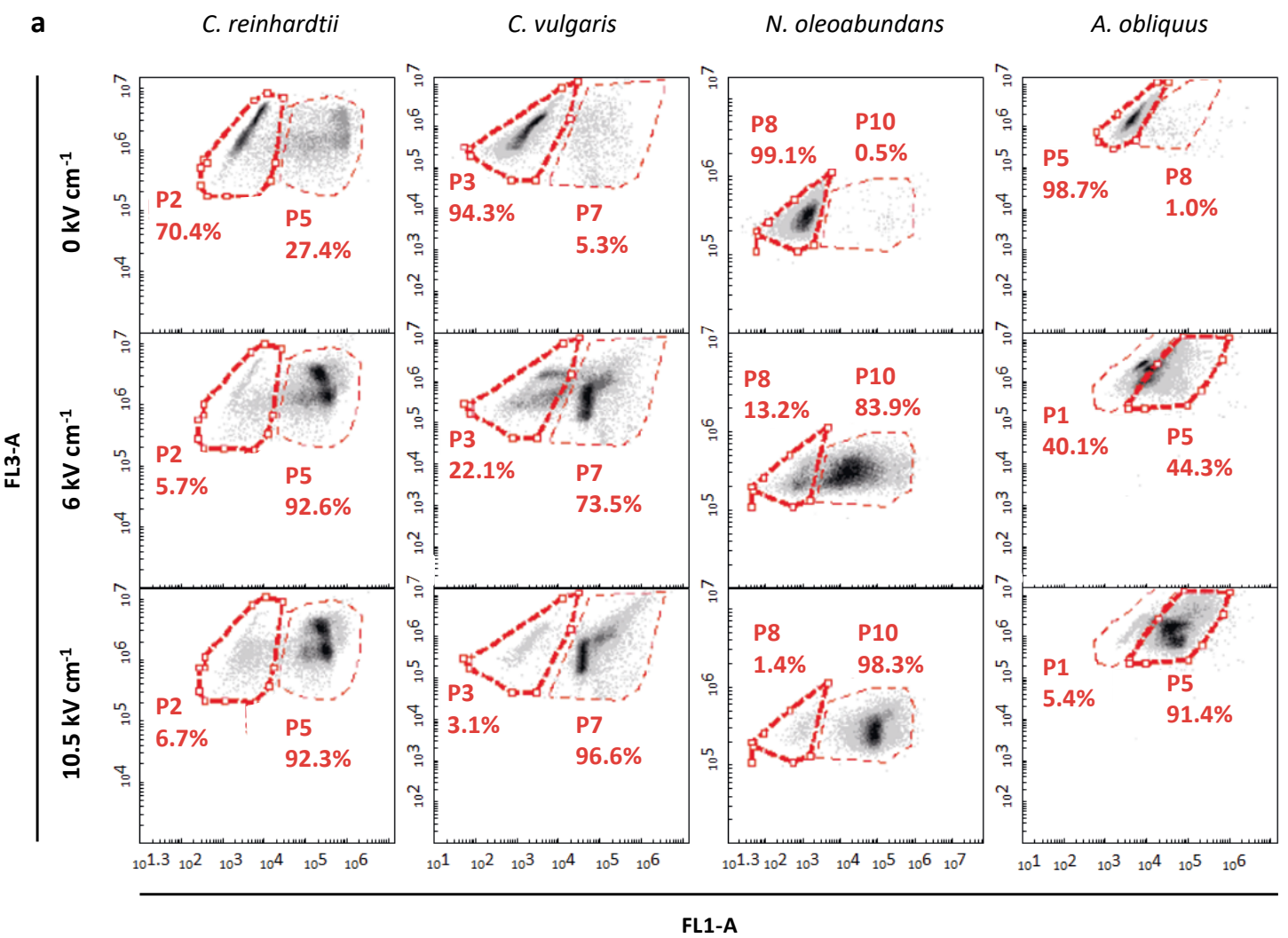




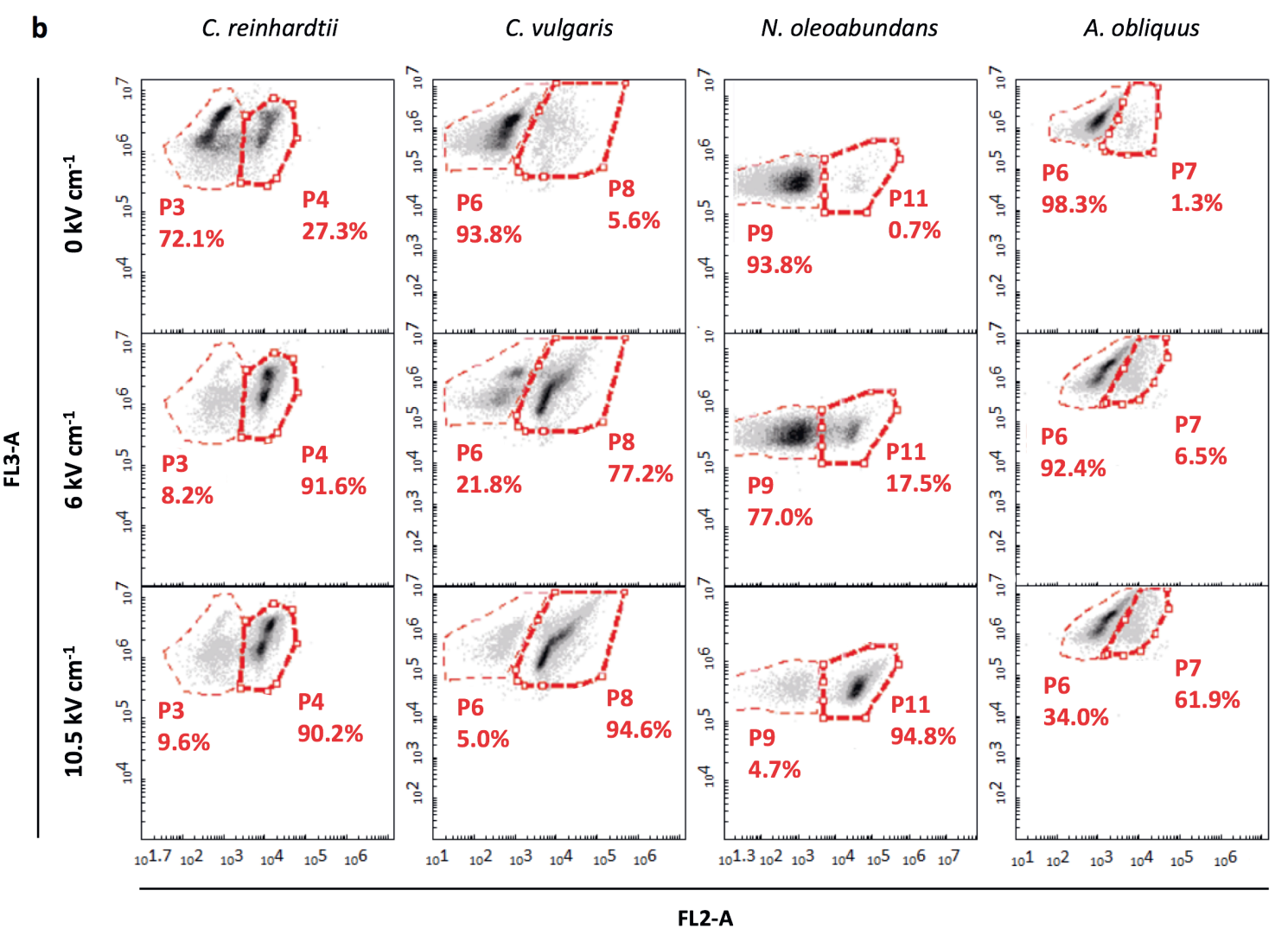





\section{Chapter 3}

\section{Stable transformation of the green algae Acutodesmus obliquus and Neochloris oleoabundans based on $E$. coli conjugation}

This chapter has been published as: Muñoz, C.F., Sturme, M.H.J., D'Adamo, S., Weusthuis, R.A., Wijffels, R.H. (2019). Stable transformation of the green algae Acutodesmus obliquus and Neochloris oleoabundans based on $E$. coli conjugation. Algal Research, 39: 101453. 


\section{Abstract}

Microalgae are an ideal platform for the production of high-value chemicals, nutritional products and biofuels. Genetic engineering could speed up the development of microalgae derived products and reduce the overall production costs. Genetic methods such as particle bombardment, electroporation, Agrobacterium tumefaciens mediated transformation (ATMT), and agitation with glass beads and silicon carbide whiskers have been developed for the genetic transformation of microalgae. However, the transformation efficiency is species dependent, so a variety of transformation methods are required to engineer a wide range of microalgae species. The oleaginous microalgae Acutodesmus obliquus and Neochloris oleoabundans have a great potential as production platforms due to their ability to produce large amounts of triacylglycerol (TAG). Genetic modification techniques however are required to increase TAG levels further or to modify the fatty acid composition. Recently, a conjugation-based method for the delivery of episomes from bacteria to diatom microalgae has been reported. In this study, we have achieved the successful transformation of green oleaginous microalgal strains by transferring an expression vector via conjugation from $E$. coli. Since delivery of exogenous DNA into the microalgae cells is only the first step in obtaining transgenic microalgae, we further analyzed transformation efficiencies by PCR and expression of the Clover fluorescent protein in the targeted species.

\section{Keywords}

Bacterial conjugation, genetic transformation, Acutodesmus obliquus, Neochloris oleoabundans, green microalgae. 


\section{Introduction}

Microalgae are considered as promising feedstock for the production of numerous valuable commercial products which can be used for the production of biofuels, cosmetics, food and feed (Caporgno \& Mathys, 2018; Khan et al., 2018; Lum et al., 2013; Sathasivam \& Ki, 2018; Wijffels et al., 2013; Wijffels \& Barbosa, 2010; N. Yan et al., 2016). In order to allow a commercial-scale production of such compounds the integral process has to be economically feasible and thus biomass and product formation need to be enhanced. Genetic modification has become a powerful approach to achieve this goal ( $\mathrm{Ng}$ et al., 2017; B. Yang et al., 2017). Since microalgal cells are not able to take up exogenous DNA by nature, several genetic techniques have been developed for this purpose. Classical mutagenesis has been used for the creation of random mutations and a more directed approach to generate specific insertions, deletions or substitutions into the host strain in order to produce the desired phenotype ( $\mathrm{Ng}$ et al., 2017).

The main bottleneck for the genetic modification of microalgae is the limited genetic toolbox that is currently available. Among transformation methods for the delivery of exogenous DNA the most common techniques are electroporation, Agrobacterium tumefaciens-mediated transformation (ATMT), ballistic systems and agitation with glass beads (K. Jeon et al., 2013; Karen L. Kindle, 1998; S. V. Kumar et al., 2004; C. Tan et al., 2005). Although, most of these techniques have been proven to work with great success in model strains such as Chlamydomonas reinhardtii, Phaeodactylum tricornutum, and Chlorella $s p$. , there is a lack of efficient and stable transformation techniques that can be applied to a broader range of microalgae strains.

Genetic engineering of microalgal strains is considered to be an obstacle due to the great diversity of species with variety of cell sizes, cell wall structures and composition (Gimpel et al., 2015). DNA delivery can be challenging since DNA has to be transferred through the cell wall, plasma membrane and nuclear membrane. Moreover, the cells have to be able to survive the chemical or 
mechanical treatment. Therefore, particular methods are needed for specific strains and thus a broader range of genetic tools have to be developed.

Electroporation became a successful genetic tool for the transformation of several microalgal species (K. Jeon et al., 2013; Muñoz et al., 2018), however the protocol optimization is often challenging and time-consuming. We previously demonstrated that microalgal species have a different resistance to transformation and thus are more or less prone to take up exogenous molecules. Moreover, viability can decrease rapidly when high voltages are applied and variations in DNA fragment lengths or macromolecule sizes can also decrease the transformation efficiencies (Muñoz et al., 2018).

Previous reports have demonstrated that the use of the bacterial pathogen Agrobacterium tumefaciens can be used as an efficient DNA delivery method in microalgae species such as Chlorella, Scenedesmus and Ankistrodesmus (Sanitha et al., 2014; Suttangkakul et al., 2019). Moreover, Karas et al. (2015)(Karas et al., 2015) and Diner et al. (2016) (Diner et al., 2016) showed that episomal plasmids containing a yeast-derived centromeric sequence CEN6ARSH4-HIS3 can be transferred by conjugation from Escherichia coli strains to the diatoms Thalassiosira pseudonana and Phaeodactylum tricornutum. When the centromeric sequence was not present in the plasmids transformation occurred by random chromosomal integration, but at a lower efficiency. The transfer of genetic material via bacterial conjugation requires mainly an origin of transfer and mob genes which are essential for the DNA mobilization. Mob genes are needed for the DNA-transfer process, they are involved in the recognition and catalysis of the cleavage at the origin of transfer site (oriT), creating a single stranded DNA that will be transferred into recipient cells. This mechanism occurs naturally between bacteria, but it has been shown to be possible between prokaryotic and eukaryotic cells as well (Lacroix \& Citovsky, 2016).

We report for the first time an efficient and stable transformation of the green microalgae Acutodesmus obliquus and Neochloris oleoabundans by transferring exogenous DNA from Escherichia coli via conjugation. We have designed and 
tested two plasmids for the integration and episomal transformation in both strains. Acutodesmus obliquus and Neochloris oleoabundans have been identified as potential candidates for the production of commercially relevant compounds. Both strains can accumulate a large amount of carbohydrates and lipids when grown under nutrient deficient conditions which makes them ideal for the production of biofuels such as bioethanol and biodiesel (Hegel et al., 2017; Mandal \& Mallick, 2009; Miranda et al., 2012; X. Sun et al., 2014). The transformation of microalgal species via bacterial conjugation offers an alternative for the delivery of exogenous DNA and reduces the cellular damage which occurs in other transformation methods. In this study, we provide a new genetic toolbox for the delivery of DNA into green microalgae which will enable the improvement of existing traits to enhance the economic potential and productivity of the studied strains.

\section{Materials and methods}

\section{Strains and growth conditions}

Acutodesmus obliquus SAG 276-6 obtained from the culture collection of algae at Göttingen University (SAG) and Neochloris oleoabundans UTEX 1185, (University of Texas) were grown in Freshwater (FW) medium as described by Breuer et al. (2012)(Breuer et al., 2012). Cultures were maintained photoautotrophically at $25^{\circ} \mathrm{C}$ on light:dark cycles of $16: 8 \mathrm{~h}$ under a light intensity of $40 \mu \mathrm{mol} \mathrm{m} \mathrm{m}^{-2} \mathrm{~s}^{-1}$ on a rotary shaker (125 rpm). Escherichia coli S17 was grown at $37^{\circ} \mathrm{C}$ in Luria-Bertani (LB) broth or agar (containing $10 \mathrm{~g} \mathrm{~L}^{-1}$ of peptone, $5 \mathrm{~g} \mathrm{~L}^{-1}$ of yeast extract and $10 \mathrm{~g} \mathrm{~L}^{-1}$ of sodium chloride) supplemented with $50 \mathrm{mg} \mathrm{L}^{-1}$ of kanamycin or $100 \mathrm{mg} \mathrm{L}^{-1}$ of ampicillin as needed.

\section{Antibiotic sensitivity test}

The sensitivity of $A$. obliquus and $N$. oleoabundans to hygromycin $B$, paromomycin and zeocin was tested. The effect of antibiotics on the viability of both strains was assessed either by plating on FW agar plates or by inoculating in FW liquid medium supplemented with the respective antibiotics. Antibiotics were added to the medium in a concentration range from 0 to $100 \mu \mathrm{gL}^{-1}$ 
when using hygromycin $B$ and paromomycin and from 0 to $50 \mu \mathrm{g} \mathrm{mL}^{-1}$ when using zeocin. An initial cell concentration of $1 \times 10^{8}$ was plated or inoculated per assay. Each test was performed in triplicate for 15-20 days and incubated at $25^{\circ} \mathrm{C}$ on light:dark cycles $(16: 8 \mathrm{~h})$ and light intensity of $40 \mu \mathrm{mol} \mathrm{m} \mathrm{m}^{-2} \mathrm{~s}^{-1}$. Flasks were continuously agitated at $125 \mathrm{rpm}$.

The effect of antibiotics in the strains studied was classified as non-inhibiting, inhibiting and completely inhibiting growth, which was determined by performing colony counting on plates and absorbance measurements at $\mathrm{OD}_{750}$ for liquid samples. We defined as non-inhibited, cultures that were able to grow, inhibited to those where no growth was observed without affecting viability and complete inhibition when viability decreased significantly compared to cultures in which no antibiotics were added to the medium.

\section{Plasmid construction}

In order to allow transfer of DNA to $A$. obliquus and $N$. oleoabundans via conjugation, two expression plasmids were constructed: pBBR1-Clover-Hyg and pBBR1-Clover-CEN (figure 1).

The conjugative plasmid pBBR1MCS-2 was obtained from Addgene and used as a backbone. pBBR1MCS-2 contains all the genes required for the plasmid transfer such as origin of transfer (oriT) and mob mobility genes. In order to allow reporter gene expression and antibiotic selection once the plasmid is transferred into the microalgae, two separate cassettes were introduced into the conjugative backbone. The gene encoding the green fluorescent protein variant Clover and the hygromycin B resistance marker aphVII were introduced into the backbone pBBR1MCS-2. The cassettes containing Clover and aphVII genes were obtained by digesting the plasmid pOpt-Clover-Hyg (Lauersen et al., 2015) with restriction enzymes KpnI and PvuI and then ligated into pBBR1MCS-2 to generate pBBR1-Clover-Hyg. Clover and aphVII gene expressions are both regulated under the $C$. reinhardtii heat shock 70A promoter/Rubisco small subunit 2 promoter (HSP70Ap/RBCS2p) and the 3' untranslated region RBCS2. The ligation reaction mixture was transformed into E. coli S17 competent cells and colonies were selected on Luria-Bertani agar 
plates supplemented with $50 \mu \mathrm{g} / \mathrm{L}$ of kanamycin. The plasmid pBBR1-CloverCEN has been constructed by introducing an additional yeast derived sequence CEN6-ARSH4-HIS3 fragment into pBBR1-Clover-Hyg plasmid. The CEN6ARSH4-HIS3 fragment was obtained by PCR amplification from the episomal plasmid pPtPuc3 (Addgene) and introduced into pBBR1-Clover-Hyg by Gibson Assembly (New England Biolabs). Primers used for the amplification of the CEN6-ARSH4-HIS3 region were F-CEN: 5'- GGCGTATTTGAAGCG GACCCGGTACGCGAGCATCACGTGCTAT-3' and R-CEN: 5'ACTAAAGGGAACAAAAGCTGGGTACGTCAAGTCCAGACTCCTGTG-3', both containing overlapping ends with pBBR1-Clover-Hyg previously linearized with restriction enzyme KpnI. The PCR conditions were as follows: Initial denaturation at $98{ }^{\circ} \mathrm{C}$ for $30 \mathrm{~s}$, denaturation at $98^{\circ} \mathrm{C}$ for $10 \mathrm{~s}$, annealing at $60.5^{\circ} \mathrm{C}$ for $30 \mathrm{~s}$ and extension at $72^{\circ} \mathrm{C}$ for $1 \mathrm{~min}$, for $35 \mathrm{cycles}$, followed by a final extension at $72{ }^{\circ} \mathrm{C}$ for $10 \mathrm{~min}$. The PCR was carried out using the proofreading Phusion DNA Polymerase (New England Biolabs).

\section{Bacterial transformation}

Escherichia coli S17 competent cells were made by using the Mix \& Go E. coli Transformation Kit and transformed with pBBR1-Clover-Hyg and pBBR1Clover-CEN plasmids according to manufacturer's instructions (Zymo Research).

\section{Transformation of Acutodesmus obliquus and Neochloris oleoabundans by conjugation}

A. obliquus and $N$. oleoabundans were exponentially grown in FW medium and harvested by centrifugation at $5000 \times \mathrm{g}$ for $5 \mathrm{~min}$ at room temperature. The cell concentrations were adjusted to $1 \times 10^{8}$ cells $\mathrm{mL}^{-1}$ and $250 \mu \mathrm{l}$ was plated onto FW medium agar plates and incubated for 7 days at $25^{\circ} \mathrm{C}$ under a $16: 8$ light:dark cycle with a light intensity of $60 \mu \mathrm{mol} \mathrm{m}^{-2} \mathrm{~s}^{-1}$. FW medium was added to the plates, the cells were scraped and the concentration was adjusted to $1 \mathrm{x}$ $10{ }^{8}$ cells $\mathrm{mL}^{-1}$ by measuring the cell number on a Multisizer III (Beckman Coulter) using a $50 \mu \mathrm{m}$ aperture tube. 
Escherichia coli S17 strains containing pBBR1-Clover-Hyg and pBBR1-CloverCEN were grown in $50 \mathrm{~mL}$ of Luria-Bertani broth at $37^{\circ} \mathrm{C}$ with constant agitation at $250 \mathrm{rpm}$ to a final $\mathrm{OD}_{600}$ of 1 . Cells were spun down for $5 \mathrm{~min}$ at $5000 \times \mathrm{g}$ and resuspended in $500 \mu \mathrm{l}$ of SOC medium.

An equal volume of $100 \mu \mathrm{l}$ of $A$. obliquus/N. oleoabundans and $E$. coli S17 culture were mixed, plated onto FW 5\% LB agar plates and incubated in the dark at $30{ }^{\circ} \mathrm{C}$ for $2 \mathrm{hrs}$. After incubation, the plates were placed into a $25{ }^{\circ} \mathrm{C}$ incubation chamber with light: dark cycles of 16:8 hrs under a light intensity of $60 \mu \mathrm{mol} \mathrm{m} \mathrm{m}^{-2} \mathrm{~s}^{-1}$ for 2 days.

Subsequently, the cells were scraped from plates and replated onto FW agar medium supplemented with $50 \mathrm{mg} \mathrm{L}^{-1}$ of hygromycin B and incubated for 15 days under conditions mentioned above.
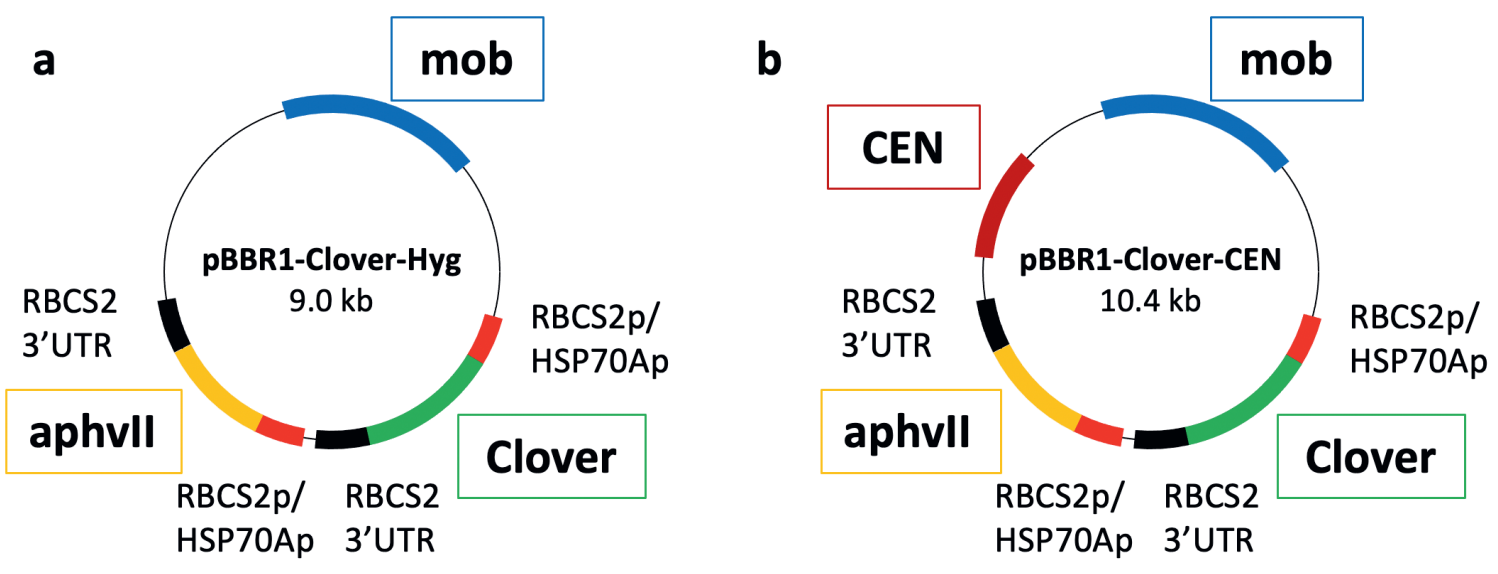

Figure 1. Schematic map of the conjugative plasmids pBBR1-Clover-Hyg (a) and pBBR1Clover-CEN (b).

\section{Optimization of transformation conditions}

In order to determine optimal conjugation conditions, we tested 3 independent incubation times. $A$. obliquus and $N$. oleoabundans were mixed and then incubated with $E$. coli at $30^{\circ} \mathrm{C}$ for 60,90 and $120 \mathrm{~min}$. In addition, we investigated differences in transformation efficiencies when mixing $1: 1$ and $1: 2$ microalgal to bacterial ratios $(\mathrm{v} / \mathrm{v})$. Microalgal strains were adjusted to $1 \times 10^{8}$ 
cells $\mathrm{mL}^{-1}$ and the bacterial inoculum was obtained from a culture with $\mathrm{OD}_{600}$ of 1.

\section{Selection of transformant lines}

Colonies were obtained from FW agar plates containing the respective antibiotics. Total genomic DNA was isolated from each colony as described by Muñoz et al. 2018 (Muñoz et al., 2018). The aphVII gene was identified in the transformant lines by performing a polymerase chain reaction using primers 5' - AGTTCCTCATCGAGAAGTTCGACAG - $3^{\prime}$ and 5' CCAGAAGAAGATGTITGCCACCTC - 3'. The PCR reaction was carried out in a total volume of $25 \mu \mathrm{L}$ with DreamTaq DNA Polymerase (Thermo Fisher). PCR conditions consisted of an initial denaturation at $95^{\circ} \mathrm{C}$ for $1 \mathrm{~min}$, followed by 35 cycles at $95^{\circ} \mathrm{C}$ for $30 \mathrm{~s}, 55^{\circ} \mathrm{C}$ for $30 \mathrm{~s}, 72{ }^{\circ} \mathrm{C}$ for 1 min and with a final extension at $72{ }^{\circ} \mathrm{C}$ for $10 \mathrm{~min}$. The PCR products were analysed on $1 \%$ agarose gel. As described by Karas et al. 2015 (Karas et al., 2015), in order to analyse episomal plasmids we transformed the extracted DNA into Escherichia coli S17 and colonies were selected on Luria-Bertani agar plates supplemented with 50 $\mu \mathrm{g} / \mathrm{L}$ of kanamycin.

\section{Fluorescent protein analysis}

The cellular fluorescence of the green fluorescent protein Clover expressed in both microalgal strains was analysed by fluorescence microscopy. Positive transformants were inoculated and grown in a 96-microwell plate containing FW medium with the respective antibiotic concentration. The transformants were incubated for 2 days at $25^{\circ} \mathrm{C}$ on light:dark cycles of $16: 8 \mathrm{~h}$ under a light intensity of $40 \mu \mathrm{mol} \mathrm{m} \mathrm{m}^{-2} \mathrm{~s}^{-1}$ on a rotary shaker (125 rpm). Detection of Clover fluorescent protein was performed with the fluorescence microscope EVOS FL Auto Cell Imaging System incorporating a GFP excitation/emission cube (EVOS FL, ThermoFisher Scientific). 


\section{$\underline{\text { Statistical analysis }}$}

All the experiments in this study were performed in biological triplicates. The data were represented as mean \pm standard deviation (SD).

\section{Results}

\section{Genetic approach for microalgae transformation via conjugation.}

In order to achieve microalgal transformation via conjugation we followed the experimental design described in figure 2. We performed an antibiotic sensitivity test to select appropriate selection markers to be used for the identification of transformant lines. We evaluated the effect of hygromycin $B$, paromomycin and zeocin on the cell growth of $A$. obliquus and $N$. oleoabundans. To perform the sensitivity test, we either plated or inoculated the strains in FW agar plates or FW liquid medium supplemented with the respective antibiotics. To investigate whether conjugation can be used to transfer genes between $E$. coli and microalgae we constructed the plasmids pBBR1-Clover-Hyg and pBBR1-Clover-CEN. Both conjugative plasmids contain an origin of transfer (oriT), mob genes which are required for the DNA mobilization, the Clover gene as a green fluorescent reporter and an antibiotic resistance gene which confers resistance to the selected antibiotic. In order to obtain episomal replication in A. obliquus and $N$. oleoabundans we constructed and delivered the plasmid pBBR1-Clover-CEN. Finally, both plasmids were transferred to the conjugative strain $E$. coli S17. Bacterial and microalgal cells were co-cultivated first on liquid medium and subsequently plated on agar plates containing 95\% (V/V) FW medium and $5 \%(\mathrm{v} / \mathrm{v})$ LB medium. After 2 days of incubation the plates were scraped and re-plated on FW agar plates containing the selected antibiotic. All antibiotic resistant colonies were analysed by PCR amplification of the antibiotic resistant gene and analysis of the green fluorescent Clover by microscopy. 


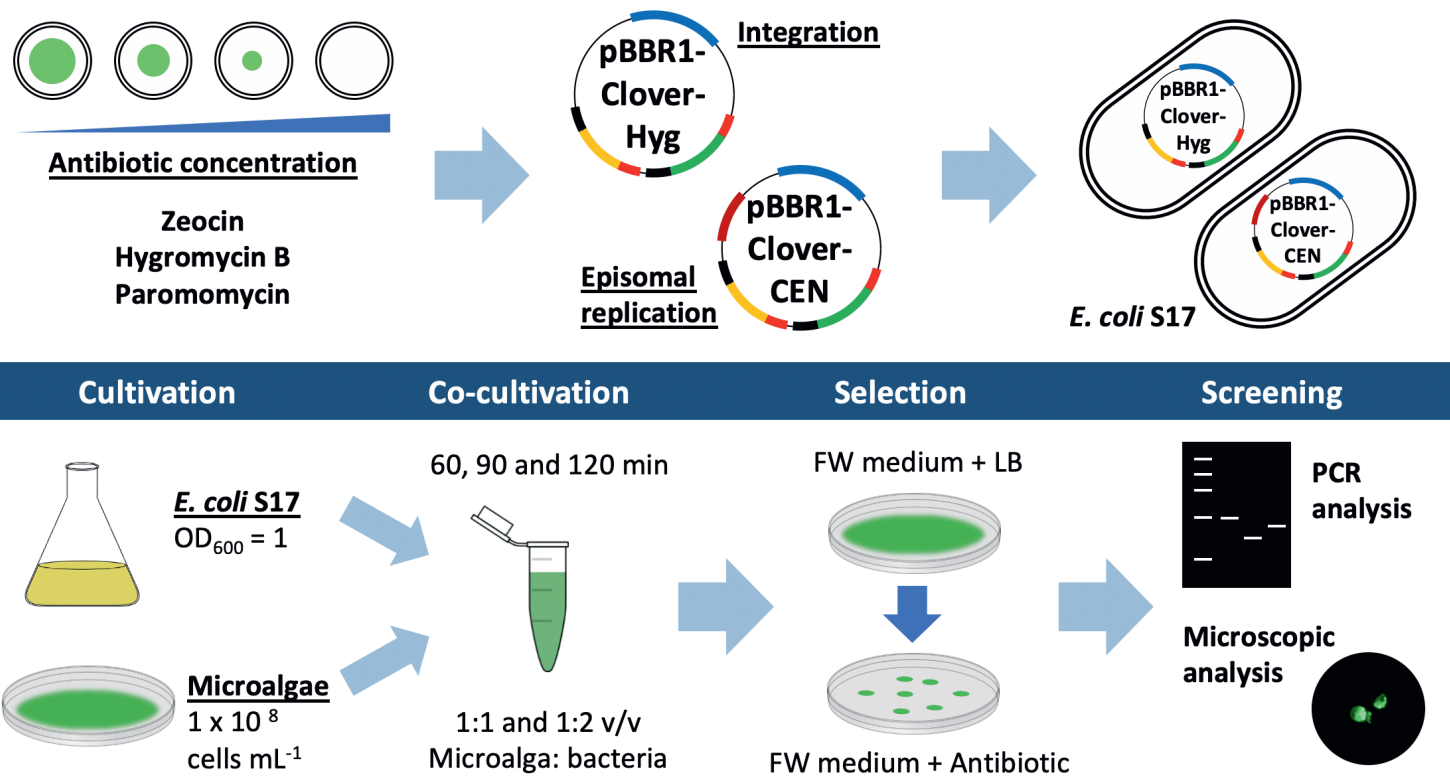

Figure 2. Experimental design for the genetic transformation of $A$. obliquus and $N$. oleoabundans via bacterial conjugation.

\section{Effect of antibiotics on Acutodesmus obliquus and Neochloris oleoabundans}

Our tests showed that all 3 antibiotics resulted in complete growth inhibition of both strains when cells were exposed to concentrations over $50 \mu \mathrm{g} \mathrm{mL}^{-1}$. As shown in table 1, liquid media containing the antibiotics were more effective at inhibiting growth of both microalgae species compared to the effect observed on plates supplemented with equal antibiotic concentrations. $N$. oleoabundans showed to be highly inhibited by all 3 antibiotics when the test was performed in liquid medium even at very low concentrations. Cell growth of both species was strongly inhibited by zeocin. When zeocin was used either inhibition or no survival was observed at the lowest concentrations tested. The results indicate that the antibiotics tested are suitable and can be employed as selection markers. Hygromycin B at a concentration of $50 \mu \mathrm{g} \mathrm{mL}^{-1}$ showed a similar effect in both strains, which is sufficient to completely inhibit growth. The aphVII gene 
conferring resistance to hygromycin B was chosen and included in the expression plasmids for the selection of positive transformants.

Table 1. Antibiotic sensitivity test performed on $A$. obliquus and $N$. oleoabundans. The effect of hygromycin B, paromomycin and zeocin were tested on FW agar plates $(\mathbf{P})$ and FW liquid medium ( $\mathbf{L}$ ). The response of both strains to different antibiotic concentrations were classified as non-inhibited (+), inhibited (-) and complete growth inhibition (--). Hyg., hygromycin, Par., paromomycin, Zeo., zeocin. ND., not determined. The data represents the average of $n=3$ replicate experiments.

\begin{tabular}{|c|c|c|c|c|c|c|c|c|c|c|c|c|}
\hline \multirow{3}{*}{$\begin{array}{l}\text { Conc. } \\
\left(\mu \mathrm{gL}^{-1}\right)\end{array}$} & \multicolumn{6}{|c|}{ Acutodesmus obliquus } & \multicolumn{6}{|c|}{ Neochloris oleoabundans } \\
\hline & \multicolumn{2}{|c|}{ Hyg } & \multicolumn{2}{|c|}{ Par } & \multicolumn{2}{|c|}{ Zeo } & \multicolumn{2}{|c|}{ Hyg } & \multicolumn{2}{|c|}{ Par } & \multicolumn{2}{|c|}{ Zeo } \\
\hline & $P$ & L & $P$ & L & $P$ & $\mathrm{~L}$ & $P$ & $\mathrm{~L}$ & $\mathrm{P}$ & $\mathrm{L}$ & $P$ & L \\
\hline $\mathbf{0}$ & + & + & + & + & + & + & + & + & + & + & + & + \\
\hline 5 & + & + & + & + & - & - & + & - & - & - & - & - \\
\hline 10 & + & - & + & - & - & -- & + & - & - & -- & -- & -- \\
\hline 20 & - & - & + & -- & -- & -- & - & -- & -- & -- & -- & -- \\
\hline 30 & - & - & - & -- & -- & -- & - & -- & -- & -- & -- & -- \\
\hline 40 & -- & -- & - & -- & -- & -- & -- & -- & -- & -- & -- & -- \\
\hline 50 & -- & -- & -- & -- & -- & -- & -- & -- & -- & -- & -- & -- \\
\hline 75 & -- & -- & -- & -- & ND & ND & -- & -- & -- & -- & ND & ND \\
\hline 100 & -- & -- & -- & -- & ND & ND & -- & -- & -- & -- & ND & ND \\
\hline
\end{tabular}




\section{Transformation of Acutodesmus obliquus and Neochloris oleoabundans via conjugation}

In our first attempt we performed conjugation by co-cultivating during 60 min, using a 1:1 ratio microalga:bacteria $(\mathrm{v} / \mathrm{v})$. We obtained colonies after 15 days of incubation on FW agar plates containing hygromycin B as a selective pressure. Around 56 and 52 hygromycin resistant colonies were obtained on plates, however only $10-14 \%$ were successfully identified as positive transformants for $N$. oleoabundans and $A$. obliquus colonies, respectively. To determine the optimal conjugation times and thus increase the transformation efficiencies, we investigated 3 different incubation times of microalgal strains with the donor strain. The experiment was performed by mixing $1 \times 10^{8}$ microalgae cells with the bacterial strain at $\mathrm{OD}_{600}$ of 1 . The mixture was incubated at $30{ }^{\circ} \mathrm{C}$ for 60,90 and $120 \mathrm{~min}$. As shown in figure 3, longer incubation times resulted in more colonies on plates. Around 200 and 150 hygromycin B resistant colonies were obtained when $A$. obliquus and $N$. oleoabundans, respectively, were exposed to the longest conjugation time. The presence of hygromycin B resistance gene aphVII in the transformant lines was confirmed by PCR analysis. $A$. obliquus exposed to 120 min of co-cultivation with $E$. coli, resulted in $20 \%$ of positive transformants from the total colonies on plates. Co-cultivation with $N$. oleoabundans resulted in $17 \%$ positive transformants. Although more positive transformants were obtained while increasing incubation times, the overall transformation efficiency was not significantly improved considering that an increasing number of colonies not containing the aphVII gene were obtained on the selective medium. To further investigate transformation efficiencies, we performed the transformation for 120 min by using $1: 1$ and $1: 2(\mathrm{v} / \mathrm{v})$ microalgae to bacteria ratios. When using $1: 2$ ratios we observed a higher variability in number of colonies and positive transformants among the triplicate plates but not a significant increase in the transformation efficiencies. 
We also performed the transformation of $A$. obliquus and $N$. oleoabundans by transferring the plasmid pBBR1-Clover-CEN via conjugation (figure $\mathbf{3 e - h}$ ). No significant differences were observed compared to the results obtained with the plasmid pBBR1-Clover-Hyg (figure 3a-d). Furthermore, in order to analyse episomal replication we performed the transformation of $E$. coli competent cells with the extracted genomic DNA from transformant lines, however no bacterial colonies were obtained on the selective plates. Since we were not able to recover the episomal plasmid from $A$. obliquus and $N$. oleoabundans strains
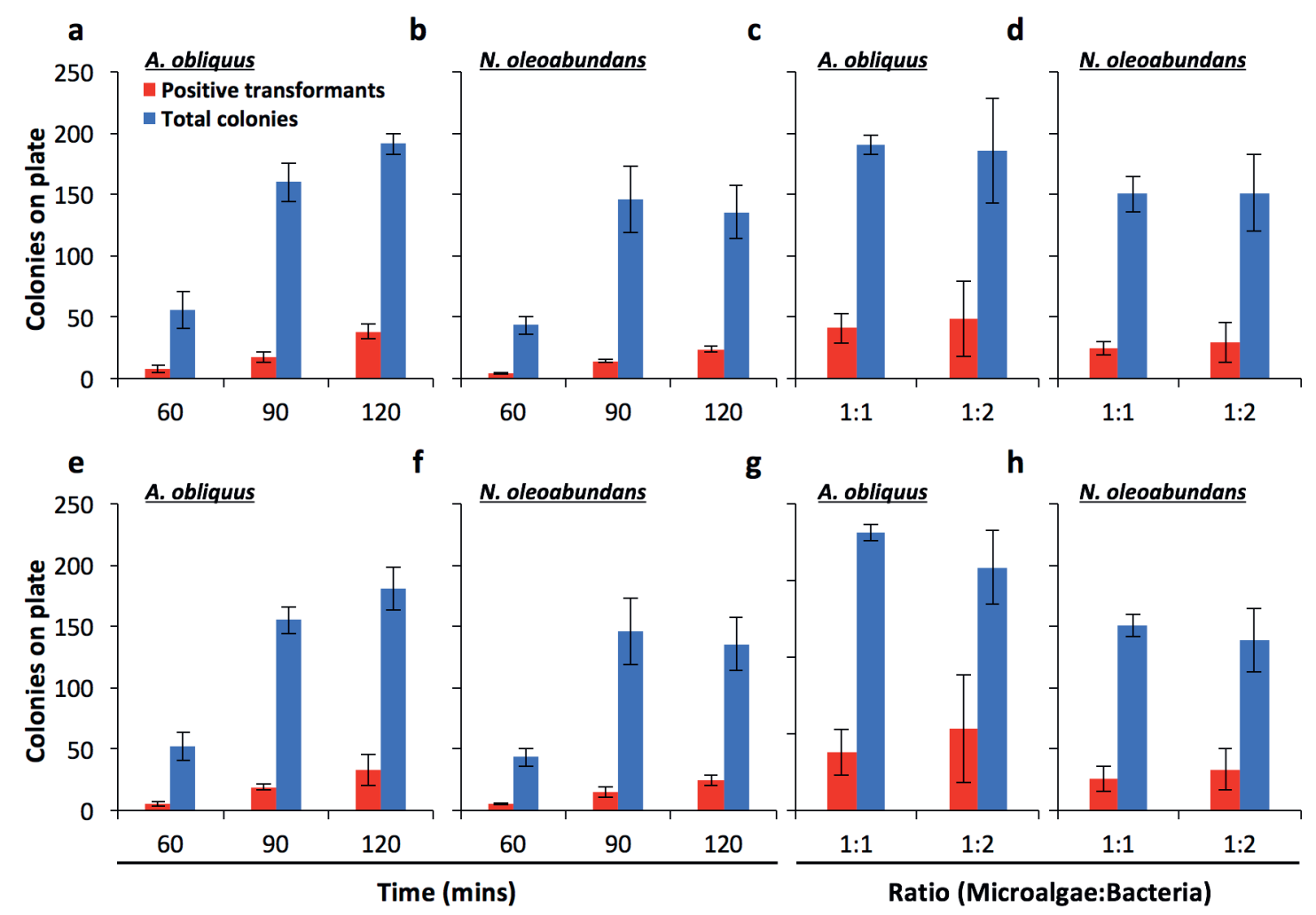

Figure 3. Number of colonies obtained per transformation at different conjugation times and ratios microalgae:bacteria. $A$. obliquus and $N$. oleoabundans colonies obtained on plates when transformed with pBBR1-Clover-Hyg (a-d) and pBBR1Clover-CEN (e-h) under different conjugation times (a, $\mathbf{b}, \mathbf{e}$ and $\mathbf{f})$. Ratios of $1: 1$ and 1:2 microalgae:bacteria were tested when exposed to $120 \mathrm{~min}$ of conjugation $(\mathbf{c}, \mathbf{d} \mathbf{~ g}$ and $\mathbf{h}$ ). Positive transformants correspond to colonies containing the aphVII gene. The data represents the mean \pm standard deviation (SD). The data represent the average of $\mathrm{n}=3$ replicate experiments. Standard deviation bars are shown. 
containing the hygromycin resistance gene, indicating that random integration of the plasmid in the genome might have occurred.

\section{Expression of fluorescent reporter gene}

The green fluorescent protein Clover was used as a reporter to evaluate gene expression. We selected and analysed 10 positive transformants from each strain that were confirmed to contain the aphVII resistance gene after transformation with either pBBR1-Clover-Hyg or pBBR1-Clover-CEN. As shown in figure 4, the fluorescence emission of Clover protein is visualized as green and the auto-fluorescence from the chloroplast as red. The red autofluorescence of the chloroplast in the transformants was similar to the fluorescence observed in the wild type strains. The green fluorescence of Clover was clearly observed in the transformants and only a vague green fluorescence background is visualized in the wild types. Although the Clover reporter gene was stably and successfully expressed in all positive transformants, we visualized different fluorescence intensities in all the strains tested. Very high fluorescence signal was observed in transformants N.O.ST-1, N.O.ST-3, N.O.ST5 and A.o.ST-4 compared to the wild type strains.

\section{Stability of transformants}

The strains N.o.ST-1, N.o.ST-3, N.o.ST-5 and A.o.ST-4 which showed high green fluorescence emissions were selected, inoculated in antibiotic-free medium and grown for 8 weeks. All the strains were re-inoculated each week to new fresh medium and transferred to FW agar plates in the last week. To analyze the stability of the transformed cell lines we performed the extraction of genomic DNA from colonies obtained on plates and we analyzed them by PCR amplification of the selective marker. The aphVII gene was detected at the expected size in all the tested transformants but the wild type strain. Moreover, no differences in green fluorescence were observed when analyzed with a fluorescent microscope. 


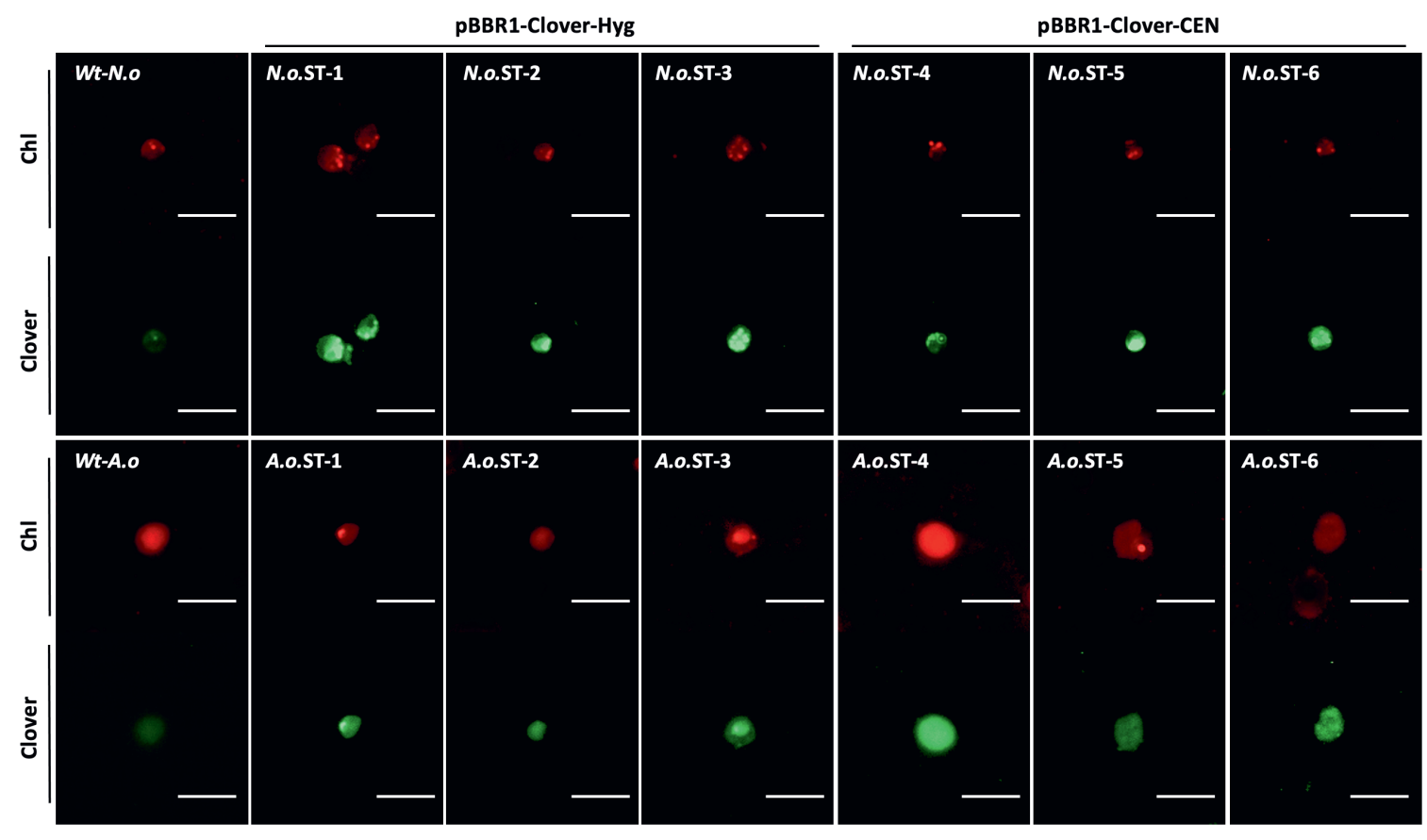

Figure 4. Microscopic visualization of red chloroplast auto-fluorescence (Chl) and detection of green fluorescent protein Clover (Clover) in A. obliquus and $N$. oleoabundans strains (ST) transformed with pBBR1-Clover-Hyg and pBBR1-Clover-CEN plasmids. $A$. obliquus and $N$. oleoabundans wild-type strains (Wt-A.o and Wt-N.o) were used as negative controls. All scale bars $=10 \mu \mathrm{m}$.

\section{Discussion}

The genetic transformation of $A$. obliquus and $N$. oleoabundans was previously achieved by Guo et al. (2013) and Chungjatupornchai et al. (2016) respectively, by using an electroporation method to deliver the exogenous DNA. The major drawbacks of microalgal transformation methods such as electroporation and ballistics are the need of specialized equipment and the poor transformation efficiency when relatively long DNA fragments are delivered into the microalgae cells (S. Kang et al., 2015; Muñoz et al., 2018). Moreover, they can result in high cell death since the cells are not able to recover after the treatment is applied (Muñoz et al., 2018). Therefore, having a broader range of genetic tools available could offer a wider spectrum of capabilities. Bacterial conjugation is a natural mechanism for the gene transfer between bacterial strains. This mechanism has been extensively used as a transformation method for the delivery of cloning and expression vectors to 
other prokaryotic and eukaryotic cells (Lacroix \& Citovsky, 2016). In the current study, we adapted and optimized the conjugation conditions for the transformation of the green algae $A$. obliquus and $N$. oleoabundans. Firstly, we determined an appropriate selection marker for both microalgae strains. To identify the correct markers which would allow us to select positive transformants, we performed an antibiotic sensitivity test on plates and liquid medium supplemented with hygromycin B, paromomycin and zeocin. Our results revealed that all the antibiotics tested were effective as selective agents. Hygromycin B was chosen as a suitable selectable marker for both strains. We demonstrated that $50 \mu \mathrm{g} \mathrm{mL}^{-1}$ is the lowest concentration needed to cause cell death in both strains which is a crucial factor for the selection of positive transformants. Hygromycin $B$ has been reported to be an effective antibiotic for the selection of $A$. obliquus and $N$. oleoabundans strains carrying the aphVII gene (Chungjatupornchai et al., 2016; Guo et al., 2013).

In order to perform genetic transformation via conjugation the plasmids pBBR1-Clover-Hyg and pBBR1-Clover-CEN were constructed. The conjugative plasmids carry an origin of replication and a kanamycin resistance gene which allows replication and maintenance of the plasmid in the $E$. coli strain. In addition, both plasmids contain an origin of transfer and mob genes which are required for the DNA mobilization from the bacterial donor to microalgal cells (Karas et al., 2015). Furthermore, we introduced the aphVII gene which confers resistance to hygromycin $B$ and the green fluorescent protein Clover gene under the regulation of the $C$. reinhardtii heat shock 70A promoter/Rubisco small subunit 2 promoter (HSP70Ap/RBCS2p) and the $3^{\prime}$ untranslated region of RBCS2.

The plasmids were successfully delivered via bacterial conjugation to both $A$ obliquus and $N$. oleoabundans strains. Positive transformants were identified by the amplification of the aphVII gene from genomic DNA, which indicates the integration of the plasmids in the microalgal genomes. We have achieved a higher percentage of positive transformants with $A$. obliquus than $N$. oleoabundans regarding the total colonies obtained on the selective plates. We hypothesized that the interaction microalgae:bacteria might be different when 
transferring DNA through different cellular barriers considering that both microalgae vary in cell size, cell wall structure and composition (Allard et al., 1998; D. Wang et al., 2015). In our attempts to increase transformation efficiencies we were able to obtain more positive transformants per plate by increasing the conjugation times. These results show that a longer period of time of exposure of microalgae to bacterial cells provides more time for the DNA transfer to take place. However, the efficiencies were not increased significantly considering that an increasing number of positive and falsepositive strains were obtained simultaneously on plates. On the other hand, when microalgae were exposed to twice the volume of bacterial culture, we found high variations in positive and false-positives ratios among the triplicate plates. We suspect that higher biomass plated on the selective medium can result in different exposures to light and the antibiotic, which could lead to higher variabilities as observed in our results.

In order to obtain episomal replication in $A$. obliquus and $N$. oleoabundans we constructed and delivered the plasmid pBBR1-Clover-CEN into both strains. Although successful transformation was achieved, we were not able to recover the plasmid from the positive transformants. All transformant lines were confirmed to contain the antibiotic resistance marker even after 8 weeks of incubation in antibiotic-free medium. In previous work using episomal plasmid transformation conducted in Nannochloropsis oceanica and P. tricornutum, episomal plasmids were rescued; moreover, subculturing the cells in antibioticfree medium and growing them for 10 and 30 days, respectively, was an efficient strategy to lose the episomal plasmids (Diner et al., 2016; Poliner et al., 2018; Slattery et al., 2018). Thus, since we could not rescue any episomal plasmids from genomic extraction and we have seen stable transgene expression subculturing cells in antibiotic-free medium for 8 weeks, our results suggest that chromosomal integration events may have occurred, as observed with the non-episomal plasmid pBBR1-Clover-Hyg. The centromere sequence used in this study has been specifically identified and adapted for diatom microalgae strains. Diner et al. (2016)(Diner et al., 2016) demonstrated that several combinations of the elements present in the centromere sequence and variations in GC content can lead to higher transformation efficiencies in 
Phaeodactylum tricornutum. Therefore, a more appropriate centromeric sequence has to be identified to allow episomal maintenance and replication in A. obliquus and $N$. oleoabundans.

To evaluate the expression of the positive transformants we used the GFPvariant Clover as reporter gene for its high fluorescence emissions and photostability (Lam et al., 2012). All positive transformants tested showed the expression of the reporter gene. We visualized different fluorescence intensities due to random integration of our expression vectors. It is likely that multiple integration or integration positional effects may be causing different expression levels (Doron et al., 2016).

Our work demonstrated for the first time that it is possible to transform green microalgae via $E$. coli conjugation. We reported stable transgene expression in two different species, moreover we performed optimization of the transformation protocol. The development of this novel protocol offers advantages over traditional methods for its simplicity, no need of expensive equipment such as electroporator or gene gun, minimal disruption of algae cells and transfer of relatively large plasmids which could allow the expression of large set of genes. This method therefore can facilitate metabolic engineering approaches for the creation of green microalgae with improved production traits.

\section{Acknowledgements}

This research project is financially supported by the National Commission of Scientific and Technologic Research of Chile (CONICYT).

\section{Author's contribution}

CFM and MHJS designed the experiments. CFM performed the experiments, analyzed, interpreted the data and wrote the manuscript. MHJS, RAW, SD and RHW supervised the project and edited the manuscript. All authors contributed to the work, discussed the results, read and approved the final version of this manuscript. 


\section{Conflict of interest statement}

We declare that there are no conflicts of interest related to this work.

\section{Statement of informed consent, human/animal rights}

No conflicts, informed consent, human or animal rights applicable. 



\section{Chapter 4}

\section{Effect of single and combined expression of $L P A T, G P A T$ and $D G A T$ on lipid accumulation and composition in}

Neochloris oleoabundans

This chapter has been published as: Muñoz, C.F., Weusthuis, R.A., D'Adamo, S., Wijffels, R.H. (2019). Effect of single and combined expression of LPAT, GPAT and DGAT on lipid accumulation and composition in Neochloris oleoabundans. Frontiers in Plant Science, 10: 1573. 


\section{Abstract}

Microalgal lipids are promising feedstocks for food and biofuels. Since lipid production by microalgae is not yet economically feasible, genetic engineering is becoming a promising strategy to achieve higher lipid accumulation and productivities. Enzymes involved in the Kennedy pathway such as glycerol-3phosphate acyltransferase (GPAT), lysophosphatidic acid acyltransferase (LPAT) and diacylglycerol acyltransferase (DGAT) catalyze key steps in the formation of triacylglycerol, which is the main constituent of lipids in $N$. oleoabundans. The overexpression of these enzymes in the targeted strain has a great potential to further increase their triacylglycerol content.

We overexpressed single and multiple encoding genes for LPAT, GPAT and DGAT from Acutodesmus obliquus in N. oleoabundans. Strains overexpressing single genes produced up to $52 \%$ and $45 \% \mathrm{~g} \cdot \mathrm{gDW}^{-1}$, which corresponds to 1.3 and 1.4-fold increase in total fatty acids and triacylglycerols, respectively. The orchestrated expression of the three genes resulted in $49 \%$ and $39 \% \mathrm{~g}$. $\mathrm{gDW}^{-1}$, which is 1.2-folds increase in total fatty acids and triacylglycerols. Single expression of LPAT, GPAT and DGAT genes resulted in higher lipid productivities during starvation without a significant effect on growth and photosynthetic activity during replete conditions. On the other hand, the simultaneous expression of LPAT, GPAT and DGAT genes resulted in 52\% lower growth rate, $14 \%$ lower photosynthetic activity and 4-folds increase in cell diameter. Moreover, the multigene expressing line showed a decrease in carbohydrates and protein content and an increase in pigments during nitrogen starved condition.

The single and multiple expression of heterologous genes LPAT, GPAT and DGAT showed to significantly enhanced the lipid accumulation in $N$. oleoabundans. Single gene expression resulted in higher lipid production and productivities without having a significant impact in the physiological status of the strains. This approach shows the potential for the generation of microalgal strains with higher economical potential for the production of lipids. 


\section{Keywords}

Biodiesel, triacylglycerol, Neochloris oleoabundans, green microalgae, glycerol3-phosphate acyltransferase (GPAT), lysophosphatidic acid acyltransferase (LPAT), diacylglycerol acyltransferase (DGAT). 


\section{Introduction}

The continuous growth of the world population is increasing the global demand for food and fuel. Current oil production derives mainly from oleaginous crops and non-renewable fossil reserves, whose utilization causes a negative environmental impact (Alptekin et al., 2014; Majidian et al., 2018; Moody et al., 2014). It has been shown that the use of biodiesel over fossil fuels decreases greenhouse gas emissions and crop cultivation for fuel production creates a direct competition with food and feed production (Gharabaghi et al., 2015; Hanaki \& Portugal-Pereira, 2018). Additionally, agricultural crop cultivation for oil production requires large amounts of arable lands and freshwater. In the last few decades, microalgae have attracted attention for their potential to become a sustainable renewable feedstock for food and fuel production (Griffiths \& Harrison, 2009; Medipally et al., 2015). Microalgae are aquatic photosynthetic microorganisms, thus capable to use sunlight and water to fix $\mathrm{CO}_{2}$ into biomass. Unlike higher plants, they do not need arable land and have significantly higher biomass yields and productivities (Brennan \& Owende, 2010; Remmers et al., 2018). Moreover, some microalgal species can accumulate large amounts of triacylglycerol (TAG) as storage compounds, which can be converted into biodiesel by transesterification (Benvenuti et al., 2017; Breuer et al., 2012; Faried et al., 2017).

Oleaginous microalgae strains such as Neochloris oleoabundans can produce TAG up to $44 \%$ of their dry weight (Breuer et al., 2012). Since high production costs are currently involved in the process, yields and productivities have to be improved in order to allow for full commercial scale production (Benvenuti et al., 2017; Ruiz et al., 2016). Several strategies have been proposed for their great potential to reduce the elevated costs. Efficient cultivation systems and optimization of processes and growth conditions could reduce operational costs. Additionally, costs can be reduced by selection of microalgal strains with enhanced productivities or the use of genetically improved strains which can be achieved by laboratory evolution, random mutagenesis and direct genetic manipulation (Radakovits et al., 2010; Remmers et al., 2018). Recent advances in genetic engineering and the development of new genetic tools have allowed 
the genetic modification of microalgae, increasing our understanding in the fatty acid biosynthesis pathways (Radakovits et al., 2010; Vazquez-Villegas et al., 2018). Several studies have shown that genes encoding key enzymes in the Kennedy pathway are potential candidates for genetic engineering to enhance TAG productivities (Banerjee et al., 2016; $\mathrm{Ng}$ et al., 2017). The Kennedy pathway is responsible for the synthesis of triacylglycerols in the endoplasmic reticulum (figure 1). TAG synthesis starts with the acylation of glycerol-3-phosphate (G3P) by glycerol 3-phosphate acyltransferase (GPAT) to form lyso-phosphatidic acid, which is further converted into phosphatidic acid by lysophophatidic acid acyltransferase (LPAT). phosphatidic acid phosphatase (PAP) dephosphorylates phosphatidic acid producing diacylglycerol (DAG). Lastly, diacylglycerol acyltransferase (DGAT) catalyzes the formation of triacylglycerols using diacylglycerol and acyl-CoA as substrates (W. L. Yu et al., 2011). The Kennedy pathway intermediates phosphatidic acid (PA) and diacylglycerol (DAG) are also intermediate precursors for membrane lipids such as anionic phosphoglycerides (e.g. phosphatidyl serine), glycosylglycerides (e.g. galactosylglycerides) and zwitterionic phosphoglycerides (e.g. phosphatidyl choline, phosphatidylethanolamines) (Li-Beisson et al., 2019).

Previous studies have shown that the overexpression of genes encoding these enzymes resulted in increased lipid content. The overexpression of GPAT in Phaeodactylum tricornutum increased the neutral lipid content up to $42.6 \%$ of total lipids per dry weight, which corresponds to 2-folds increase in fatty acid content compared to wild type strain (Y. F. Niu et al., 2016). Overexpression of LPAT in Chlamydomonas reinhardtii led to $20 \%$ increase of oil content (Yamaoka et al., 2016) and an increase of 2.3-fold (up to 13\%) in TAG levels was observed when DGAT was overexpressed in P. tricornutum (Zulu et al., 2017).

In this study, we successfully expressed genes involved in the lipid biosynthesis pathway from Acutodesmus obliquus into Neochloris oleoabundans. Since Acutodesmus obliquus has been previously reported to accumulate up to $45 \%$ of total lipids per dry weight (Breuer et al., 2013), we have cloned the encoding genes GPAT, LPAT and DGAT from $A$. obliquus. In order to enhance lipid 
production in $N$. oleoabundans, we performed the single and combined gene expression of the three genes. In addition, we further investigated and characterized growth, lipid composition and cellular biochemical composition of all transformant lines obtained.

\section{Materials and Methods}

\section{Microalga strain and culture conditions}

Neochloris oleoabundans UTEX 1185 was obtained from the University of Texas Culture Collection of Algae (UTEX). N. oleoabundans strain was grown in Freshwater (FW) medium as described by Breuer et al., (2012)(Breuer et al., 2012). Prior to experiments, cultures were maintained in shake flasks at $25^{\circ} \mathrm{C}$ on light:dark cycles of $16: 8 \mathrm{~h}$ under a light intensity of $40 \mu \mathrm{mol} \mathrm{m} \mathrm{m}^{-2} \mathrm{~s}^{-1}$ on a rotary shaker $(125 \mathrm{rpm})$. During nitrogen starvation experiments, $N$. oleoabundans was grown in $500 \mathrm{~mL}$ Erlenmeyer flasks. Cultures were placed in a shaker incubator operating at $25^{\circ} \mathrm{C}$ with light intensities of $150 \mu \mathrm{mol} \mathrm{m}^{-2} \mathrm{~s}^{-1}$ (light:dark cycles of $16: 8$ ), enriched with $5 \% \mathrm{CO}_{2}$ and continuous agitation of $150 \mathrm{rpm}$.

Escherichia coli NEB-Alpha5 (New England Biolabs) was grown at $37^{\circ} \mathrm{C}$ in LuriaBertani (LB) broth or agar supplemented with $100 \mathrm{mg} \cdot \mathrm{L}^{-1}$ of ampicillin as needed.

\section{Plasmid construction}

The plasmids pUC-AcobLPAT, pUC-AcobGPAT, pUC-AcobDGAT were constructed for the single expression of LPAT, GPAT and DGAT. In order to overexpress all 3 genes simultaneously we constructed the expression vector pUC-AcobDGL. The plasmid pUC19 was obtained from Addgene and used as a backbone in all the constructs. pUC19 was digested with HindIII and NdeI restriction enzymes (Thermo Scientific) in order to linearize and remove the multiple cloning site of the vector. The cassettes CaMV35Sp-Aph7-Ter and CaMV35Sp-2A-GFP-Ter were synthetized and assembled into the linearized pUC19. Both cassettes contain the cauliflower mosaic virus (CaMV) $35 S$ 
promoter and terminator. Moreover, the cassette CaMV35Sp-2A-GFP-Ter contains a NedI restriction site between the promoter and the $2 \mathrm{~A}$ self-cleaving sequence for insertion of genes of interest. The plasmid assembly was performed using HiFi DNA Assembly Master Mix (NEB) according to the manufacturer's instructions and transformed into NEB-Alpha5 E. coli competent cells (New Englands Biolabs). The construct containing both cassettes (pUC19aph7-2A-GFP) was digested by NdeI restriction enzyme. Synthetized LPAT, GPAT and DGAT containing overlap regions with pUC19-aph7-2A-GFP were inserted via HiFi assembly to generate pUC-AcobLPAT, PUC-AcobGPAT, pUCAcobDGAT. In order to generate the plasmid pUC-AcobDGL we synthetized the sequences DGAT-2A, GPAT-2A and LPAT. All synthesized sequences contained overlap regions and stop codons of each gene were replaced by $2 A$ self-cleaving peptide of foot-and-mouth disease virus (FMDV). The 3 genes were assembled into linearized pUC19-aph7-2A-GFP by Hifi assembly and transformed into NEBAlpha5 E. coli competent cells (New Englands Biolabs). The sequences encoding for LPAT, GPAT and DGAT were obtained from the draft genome of Acutodesmus obliquus (Carreres et al., 2017) by sequence analysis (NCBI database) using Basic Local Alignment Search Tool (BLAST). All the sequences were identified as putative and no specific intracellular localization was predicted when using the prediction tool PredAlgo (Tardif et al., 2012).

\section{Transformation of $\mathbf{N}$. oleoabundans by electroporation}

N. oleoabundans cells were harvested and washed in electroporation buffer as described by Muñoz et al., (2018)(Muñoz et al., 2018). The transformation mixture containing $2 \mu \mathrm{g} \cdot \mathrm{mL}^{-1}$ of linearized plasmid and $25 \mu \mathrm{g} \cdot \mathrm{mL}^{-1}$ of boiled salmon sperm DNA (D1626, Sigma) were incubated on ice for $15 \mathrm{~min}$. The electroporation was performed in 2-mm electroporation cuvettes by applying 6 $\mathrm{kV} \cdot \mathrm{cm}^{-1}$. Electroporated cells were recovered in the dark, transferred into 10 $\mathrm{mL}$ of freshwater medium and incubated overnight on dark at $25^{\circ} \mathrm{C}$ on a rotary shaker (125 rpm). After recovery, cells were harvested, re-suspended in 200 $\mu \mathrm{L}$ of freshwater medium and plated onto freshwater agar plates containing 50 $\mu \mathrm{g} \cdot \mathrm{mL}^{-1}$ of hygromycin B. Plates were incubated at $25^{\circ} \mathrm{C}$, under light intensities 
of $60 \mu \mathrm{mol} \cdot \mathrm{m}^{-2} \mathrm{~s}^{-1}$ (light:dark cycle cycles of $16: 8$ ) and supplemented with $2.5 \% \mathrm{CO}_{2}$.

\section{Selection and screening of transformant lines}

Selection of transformant lines was performed on freshwater agar plates containing $50 \mu \mathrm{g} \cdot \mathrm{mL}^{-1}$ of hygromycin B. Antibiotic resistant colonies obtained on plate were transferred to new plates containing $75 \mu \mathrm{g} \cdot \mathrm{mL}^{-1}$ of hygromycin B. Identification of positive transformants was performed by PCR amplification of the hygromycin resistance gene ( $a p h 7)$ with primers used by Munoz et al. (2018)(Muñoz et al., 2018). DNA extraction and colony PCR were performed by using Phire Plant Direct PCR Master Mix (ThermoFisher Scientific). Colonies obtained on selective plates were transferred to $20 \mu \mathrm{l}$ of dilution buffer (provided in the kit), mixture was vortexed for 30 secs and $0.5 \mu$ was used as DNA template in the PCR reaction. Phire hot start II DNA polymerase was used for the PCR amplification of aph7 gene following the manufacturer's instructions. Positive transformants were inoculated in liquid freshwater medium containing $75 \mu \mathrm{g} \cdot \mathrm{mL}^{-1}$ of hygromycin B and grown in 48 microwell plates. Fast growing transformants were identified by optical density measurements at $750 \mathrm{~nm}$. Moreover, we visualized transformants by fluorescence microscopy and selected transformants with high green fluorescence signals for subsequent analysis. Detection of green fluorescent protein was performed with the fluorescence microscope EVOS FL Auto Cell Imaging System incorporating a GFP excitation/emission cube (EVOS FL, ThermoFisher Scientific).

\section{Growth, cell diameter, cell number and dry weight determination}

The optical density was monitored at a wavelength of $750 \mathrm{~nm}$ using a UV-VIS spectrophotometer (Hach Lange DR6000). Cell concentration and cell size were measured in triplicate with the Beckman Coulter Multisizer III (Beckman Coulter Inc., USA) using a $50 \mu \mathrm{m}$ aperture tube. Microscopic analysis was performed with the microscope EVOS FL Auto Cell Imaging System (EVOS FL, ThermoFisher Scientific). All samples were diluted 200 times in Isotone ${ }^{\circledR}$ II diluent solution before measurements. Dry weight concentration was 
determined by filtrating $10 \mathrm{~mL}$ of culture broth on a pre-dried and pre-weight $55 \mathrm{~mm}$ Whatman glass fibre filter paper (GF/F; Whatman International Ltd, Maidstone, UK). The filter was washed with filtered demineralized water and subsequently dried overnight at $100{ }^{\circ} \mathrm{C}$ before weighing. The dry weight of the samples was calculated from the difference in weight between the dry filters with and without biomass.

\section{Measurement of photosynthetic activity}

Quantum yield (Fv/Fm) was measured by chlorophyll a fluorescence at $455 \mathrm{~nm}$ using a fluorometer AquaPen-C AP-C100 (Photon Systems Instrument, Czech Republic). Samples were diluted to an $\mathrm{OD}_{750}$ of 0.5 and adapted to dark for 15 min at room temperature before measurement (Janssen et al., 2018).

\section{Determination of lipid content and fatty acid composition}

Total fatty acid content and lipid composition were determined as described by Breuer et al., (2013)(Breuer et al., 2013). Lipids were extracted from $10 \mathrm{mg}$ of freeze-dried biomass. Cells were mixed with chloroform:methanol $(1: 1.25, \mathrm{v}: \mathrm{v})$ containing tripentadecanoin (T4257, Sigma Aldrich) and 1,2-dipentadecanoyl-sn-glycero-3- phospho-(1'-rac-glycerol) (sodium salt) (840434, Avanti Polar Lipids Inc.) as internal standard for TAG and polar lipid fraction, respectively. Biomass was disrupted using a beat beater, chloroform:methanol was subsequently evaporated and the extract was dissolved in hexane:diethylether $(7: 1 \mathrm{v} / \mathrm{v})$. Neutral or apolar lipids containing triacylglycerols were separated from polar lipids using a Sep-Pak Vac silica cartridge $(6 \mathrm{cc}, 1 \mathrm{~g}$, Waters). Apolar lipids were extracted by loading $10 \mathrm{~mL}$ hexane:diethylether $(7: 1 \mathrm{v} / \mathrm{v})$ and polar lipids by loading $10 \mathrm{~mL}$ of methanol:acetone:hexane $(2: 2: 1 \mathrm{v} / \mathrm{v} / \mathrm{v})$. Both lipid extracts were quantified using a gas chromatography (GC-FID) as described by Breuer et al., (2013)(Breuer et al., 2013). The total apolar and polar lipid content was calculated as a sum of the individual fatty acids of these fractions. 


\section{Carbohydrate determination}

The total carbohydrate content was determined according to Dubois et al., (1956)(Dubois et al., 1956) and Herbert et al., (1971)(Herbert et al., 1971). Phenol-sulphuric acid was added to $10 \mathrm{mg}$ of freeze-dried microalgae and absorbance was measured at $483 \mathrm{~nm}$. Glucose monohydrate was used as a standard.

\section{Protein determination}

The total protein content was determined using a colorimetric assay (Bio-Rad DC protein assay) according to manufacturer's instructions. Total protein content was analysed in $10 \mathrm{mg}$ of freeze-dried biomass and bovine serum albumin (BSA) was used as a standard.

\section{Pigment determination}

Pigment composition was determined as described by Lichtenthaler (1987)(Lichtenthaler, 1987). The experiment was performed in triplicate and absorbance was measured at 470, 652 and $665 \mathrm{~nm}$. Chlorophyll a, chlorophyll b, total chlorophyll and total carotenoids were calculated using Arnon's equations.

\section{Calculations}

The average and maximum volumetric biomass productivities were calculated as described by Breuer et al., (2012)(Breuer et al., 2012). The growth rate was calculated using the equation $\mu=\ln \left(\mathrm{X}_{2} / \mathrm{X}_{1}\right) /\left(t_{2}-t_{1}\right)$, where $\mu$ is the specific growth rate, $X_{1}$ and $X_{2}$ are the biomass (dry weight) at time $1\left(t_{1}\right)$ and time 2 $\left(t_{2}\right)$, respectively.

\section{Statistical analysis}

All the experiments in this study were performed in biological triplicates. The data were represented as mean \pm standard deviation (SD). Statistical analysis 
was performed using the student's $t$ test and a $P$ value $<0.05$ was considered statistically significant.

\section{Results}

\section{Experimental design, plasmid construction and selection of transformants}

In order to increase triacylglycerol content in $N$. oleoabundans we constructed 4 expression vectors. The plasmids pUC-AcobDGAT, pUC-AcobGPAT and pUCAcobLPAT were constructed for the single expression of the genes encoding DGAT, GPAT and LPAT, respectively. The plasmid pUC-AcobDGL was constructed in order to assess the simultaneous expression of the 3 genes (figure 1). The expression of selectable marker (Streptomyces hygroscopicus aminoglycoside phosphotransferase gene aph7, inferring resistance to hygromycin), reporter gene (encoding green fluorescence protein, mGFP5) and genes of interest were regulated under the CaMV35S promoter and terminator. Moreover, a 2A self-cleaving peptide of foot-and-mouth disease virus (FMDV) was used for polycistronic expression of the gene(s) of interest and mGFP5, in both the single and multiple gene expression vectors. In general, viral-derived $2 \mathrm{~A}$ peptides are used for enabling a single transcript to translate discrete protein products of multiple transgenes in eukaryotes and are widely used to tie resistance markers to the production of target proteins. Having a fluorescent protein (mGFP5) at the end of the 2A polycistronic construct confers the capability to directly select for positive transformant lines. The selection for positive GFP fluorescence shows clear evidence of concomitantly expression of all the upstream genes (Daniels et al., 2014; Z. Liu et al., 2017; Poliner et al., 2018; Y. Wang et al., 2015). Around 200 hygromycin B resistant colonies were obtained on plates containing Freshwater (FW) agar medium and $50 \mu \mathrm{g} \cdot \mathrm{mL}^{-1}$ hygromycin B. Only $15 \%$ of the transformant lines survived when transferred to a higher antibiotic concentration, which were confirmed to contain the hygromycin B resistance gene aph7 (figure 1c). We then identified the fastgrowing transformants with high green fluorescence signals on liquid medium by measuring $\mathrm{OD}_{750}$ and using a fluorescence microscope, respectively, and we 
selected them for subsequent analysis. The selected positive transformants expressing the single genes DGAT, GPAT and LPAT from $A$. obliquus were named AcobDGAT+, AcobGPAT+ and AcobLPAT+, respectively. The name AcobDGL+ was assigned to the $N$. oleoabundans cell lines expressing the 3 genes simultaneously.

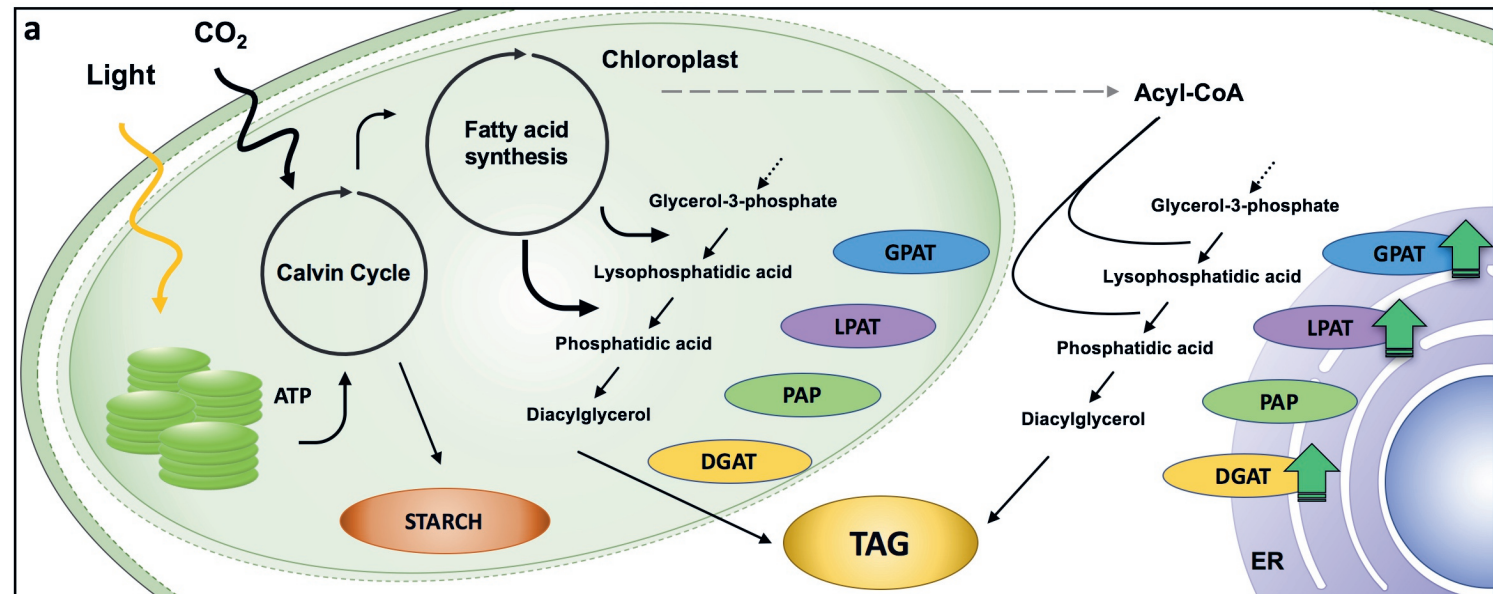

b

PUC-AcobDGAT

pUC-AcobGPAT

pUC-AcobLPAT

pUC-AcobDGL

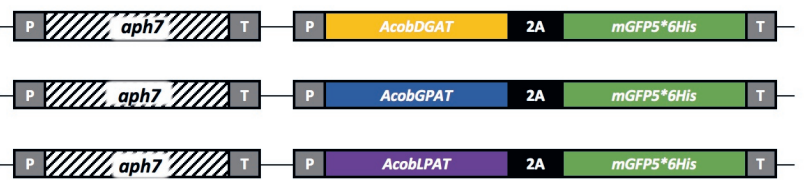

C

$537 \mathrm{bp}$ -

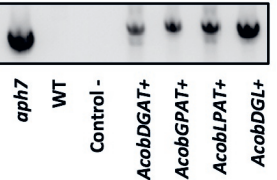

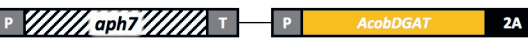

Figure 1. TAG biosynthesis pathway (a). GPAT glycerol 3-phosphate acyltransferase, LPAT lysophosphatidic acid acyltransferase, PAP phosphatidic acid phosphatase, DGAT diacylglycerol acyltransferase. Schematic representation of expression plasmids pUCAcobDGAT, pUC-AcobGPAT, pUC-AcobLPAT and pUC-AcobDGL (b). PCR amplification of aph7 gene from wild type (WT) and transformant lines (c). CaMV35S promoter (P), hygromycin B resistance gene aph7, 3' untranslated region CaMV (T) and 2A selfcleaving peptide of foot-and-mouth disease virus (FMDV).

\section{Growth analysis and characterization of engineered strains}

In order to characterize and determine the effect of gene overexpression in the selected transformant lines, we analyzed growth, morphology, and photosynthetic efficiency (figure 2). Biological replicates were cultivated in flasks under standard growth conditions and then transferred to nitrogen- 
depleted medium. During replete and deplete conditions all transformant lines showed lower cell numbers per volume compared to the wild type strain (figure 2b). Although the cell numbers were lower, AcobGPAT+ and AcobLPAT+ showed similar optical densities and dry weights compared to the wild type during nitrogen replete and deplete conditions (figure 2a-c). On the other hand, the strains AcobDGAT+ and AcobDGL+ showed significantly lower values of optical density, cell number and dry weight (end-point $P$ value $<0.05$ ).

To determine the effect of gene overexpression on photosynthetic performance we monitored the chlorophyll fluorescence $\mathrm{Fv} / \mathrm{Fm}$ of transformant lines and compared them with the $N$. oleoabundans wild type line. Compared to the control, no significant differences were observed in photosynthetic efficiency in the lines overexpressing single genes (end-point $P$ value $<0.05$ ). Wild type, AcobDGAT+, AcobGPAT+ and AcobLPAT+ showed an increase in quantum yield from 0.7 to 0.8 when grown in replete conditions, which decreased in deplete conditions to a minimum value of 0.6 at the end point of measurement. However, the three-gene overexpressing line AcobDGL+ showed a significantly lower quantum yield value (end-point $P$ value $<0.05$ ), reaching the lowest values of 0.6 during replete conditions and 0.4 during nitrogen deplete conditions (figure 2d). We further investigated the cell diameter of all transformant and wild type lines by microscopic analysis. Control and transformant lines overexpressing single genes showed no differences in cell size and during replete and deplete conditions they had cell diameters between 3.7 and $4.0 \mu \mathrm{m}$. On the contrary, AcobDGL+ was considerably larger (figure 2e), showing an average size around $14 \mu \mathrm{m}$ in cell diameter, with some cells reaching $20 \mu \mathrm{m}$ in diameter (figure $\mathbf{2 f}$ ). 

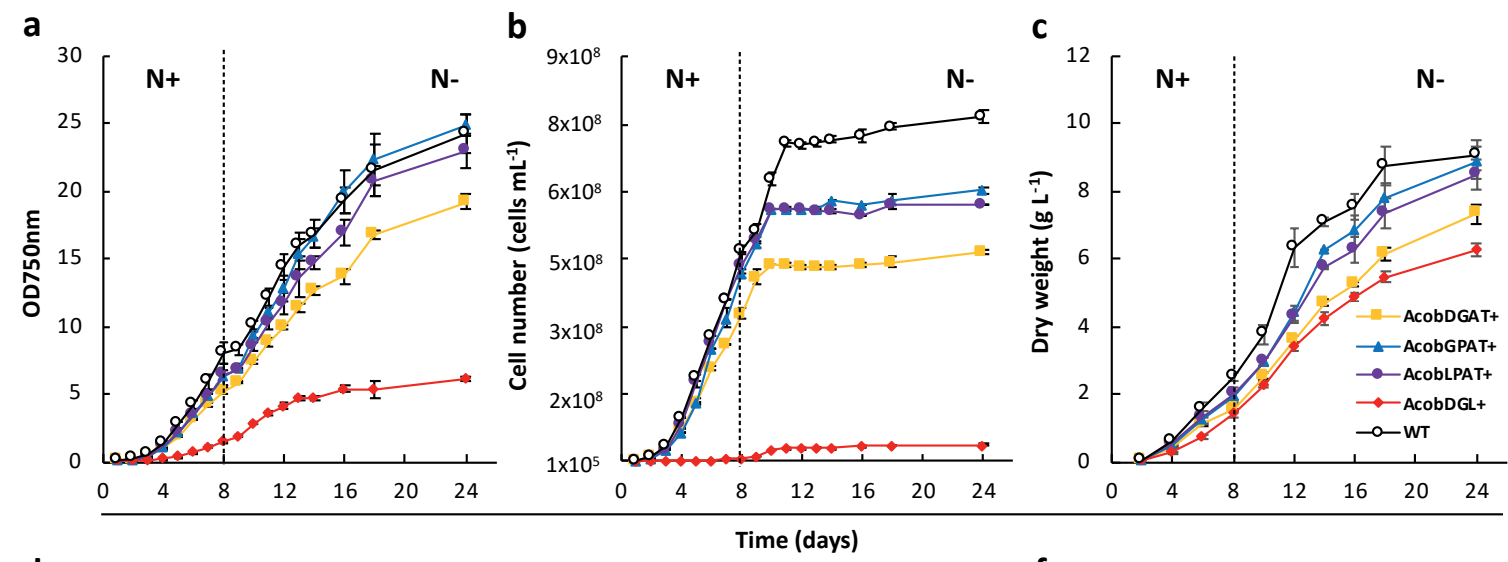

d
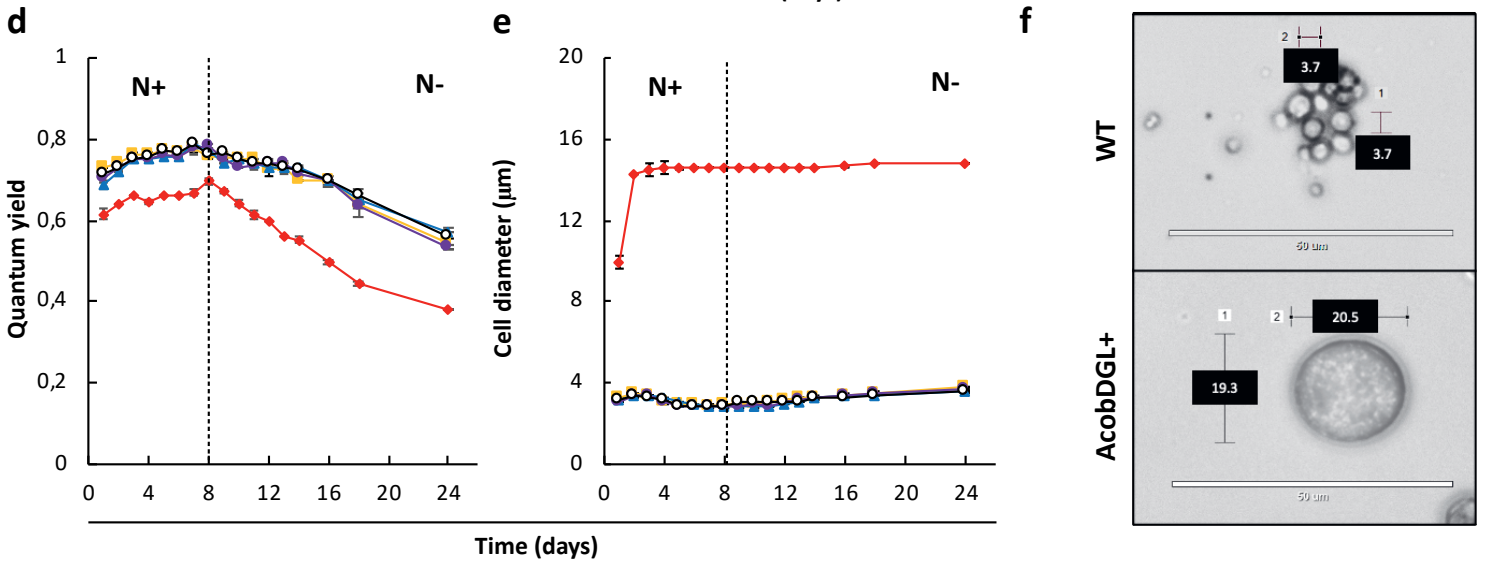

Figure 2. Analysis of cell growth and photosynthetic efficiency. Measurements of optical density at $750 \mathrm{~nm}$ (a), cell number (b), dry weight (c), quantum yield (d) and cell diameter (e) in wild type (WT) strain and transformant lines. Microscopic image of wild type strain and $N$. oleoabundans expressing DGAT, GPAT and LPAT simultaneously (f). Dashed lines indicate the moment at which nitrogen starvation started. The data represent the average of $n=3$ replicate experiments. Standard deviation bars are shown.

\section{Biochemical composition of engineered strains}

Carbohydrates, proteins and pigments were analyzed in transformant and wild type control lines during cultivation in standard and nitrogen-starved conditions.

As shown in figure 3a, no substantial differences were observed in carbohydrate content between the wild type and transformant lines during replete conditions. During deplete conditions, AcobDGL+ showed the lowest 
carbohydrate content of $0.24 \mathrm{~g} \mathrm{gDW}^{-1}$ at day 24 , while no differences were observed in the lines expressing single genes and control.

In the wild type control line, a maximum of $0.35 \mathrm{~g} \cdot \mathrm{gDW}^{-1}$ and a minimum of $0.20 \mathrm{~g} \cdot \mathrm{gDW}^{-1}$ of protein content was reached during replete and deplete conditions, respectively. Interestingly, the total protein content was higher in all transformant lines during nitrogen replete conditions and lower during starvation, compared to wild type. Furthermore, we analyzed chlorophyll a, chlorophyll b and carotenoid content at day 24 during nitrogen deplete conditions. As shown in figure 3c, AcobDGAT+ showed similar chlorophyll and carotenoid content (total pigment content of $4.5 \mathrm{mg} \cdot \mathrm{gDW}^{-1}$ ) compared to wild type. AcobGPAT+ and AcobLPAT+ resulted in lower pigment content, being AcobLPAT+ the lowest with $2 \mathrm{mg} \cdot \mathrm{gDW}^{-1}$. Interestingly, AcobDGL+ showed higher values in chlorophyll and carotenoid content reaching $7.5 \mathrm{mg} \cdot \mathrm{gDW}^{-1}$ of total pigment content compared to the control.
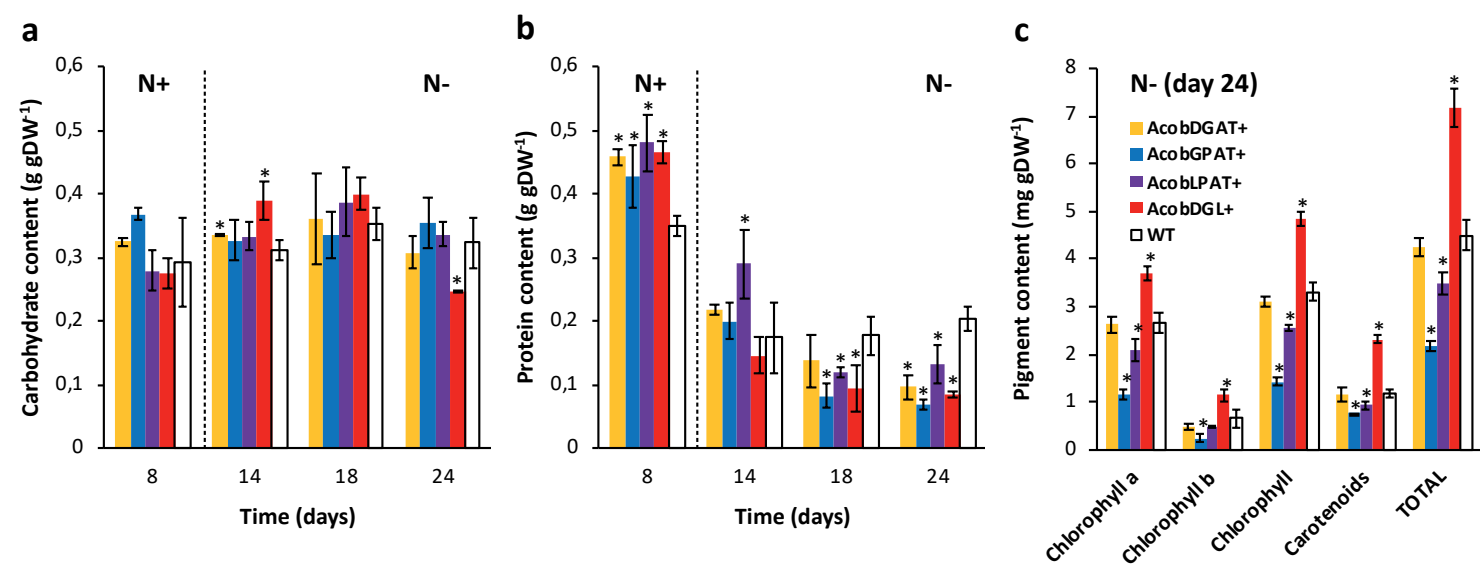

Figure 3. Biochemical composition in $N$. oleoabundans wild type strain and transformant lines. Carbohydrate (a), protein (b) and pigment (c) content in wild type (WT) and transformant lines. Dashed lines indicate the moment at which nitrogen starvation started. Pigment content as determined for all strains at day 24 under nitrogen starvation. Chlorophyll represents the sum of chlorophyll a and chlorophyll b. Total pigments correspond to the sum of total chlorophyll and carotenoids. The data represent the average of $n=3$ replicate experiments. Standard deviation bars are shown. Significant difference between wild type strain and transformant lines is indicated at $P<0.05(*)$. 


\section{Effect of gene expression on fatty acid synthesis}

The total fatty acid (TFA) and triacylglycerol (TAG) contents were analyzed throughout nitrogen replete and deplete conditions. As shown in figure $\mathbf{4 a}$, the total fatty acid content during nitrogen replete conditions was similar among transformant and wild type control lines. Only AcobDGL+ showed an increase in TAG content, having $0.9 \% \mathrm{~g} \cdot \mathrm{gDW}^{-1}$ of TAG compared to $0.1 \% \mathrm{~g} \cdot \mathrm{gDW}^{-1}$ present in AcobDGAT+, AcobGPAT+, AcobLPAT+ and wild type. The maximum TFA and TAG content was achieved at day 24 during nitrogen deplete conditions for all the transformant lines and wild type. In these conditions, the expression of the single genes encoding DGAT, GPAT and LPAT showed the highest TFA and TAG content at around $52 \%$ and $45 \% \mathrm{~g} \cdot \mathrm{gDW}^{-1}$, respectively. The simultaneous overexpression of the 3 genes in AcobDGL+ showed lower TFA and TAG ( $49 \%$ and $39 \% \mathrm{~g} \cdot \mathrm{gDW}^{-1}$ respectively) compared to the single gene expression, but higher compared to the wild type control line. We also analyzed the total fatty acids composition of the polar and apolar (TAG) fraction (figure 4b). Although the total polar lipid content of the transformant lines during replete and deplete conditions was similar to wild type, it showed differences in composition. In particular, during nitrogen replete conditions we observed an increase in C18:3 and decrease in C16:3 and C18:2 fatty acids in the polar lipid fraction for AcobDGL+, and no significant difference for the single gene expressing lines compared to wild type. During nitrogen deplete conditions, interestingly, no C20:1 was produced in AcobDGAT+, AcobGPAT+ and AcobLPAT+, while they showed an increase of C18:1 compared to wild type. Differently, AcobDGL+ showed 2.4 and 3.9-fold increase in C20:1 and C18:3 fatty acids content, respectively, with a reduced $\mathrm{C} 16: 0, \mathrm{C} 18: 1$ and $\mathrm{C} 18: 2$ content, compared to wild type. Similarly, also the apolar (TAG) fraction presented differences in composition. During replete conditions, AcobDGL+ showed an increase in C18:1 and the presence of $\mathrm{C} 18: 4$ and $\mathrm{C} 16: 1$. For the strains expressing single genes we observed a substantial decrease in C16:2 and increase in C18:2 in the apolar (TAG) fraction. Moreover, AcobDGAT+, AcobGPAT+ and AcobDGL+ showed the presence of 16:3, and AcobDGAT+, AcobLPAT+ and AcobDGL+ had higher C16:0 content compared to wild type. Under deplete conditions, the apolar lipid composition of the transformant lines 
expressing single genes showed a similar distribution compared to wild type, while AcobDGL+ showed an increase in C18:3, C18:1, and C20:1 fatty acids and a decrease in C16:0 and C18:2 fatty acids.
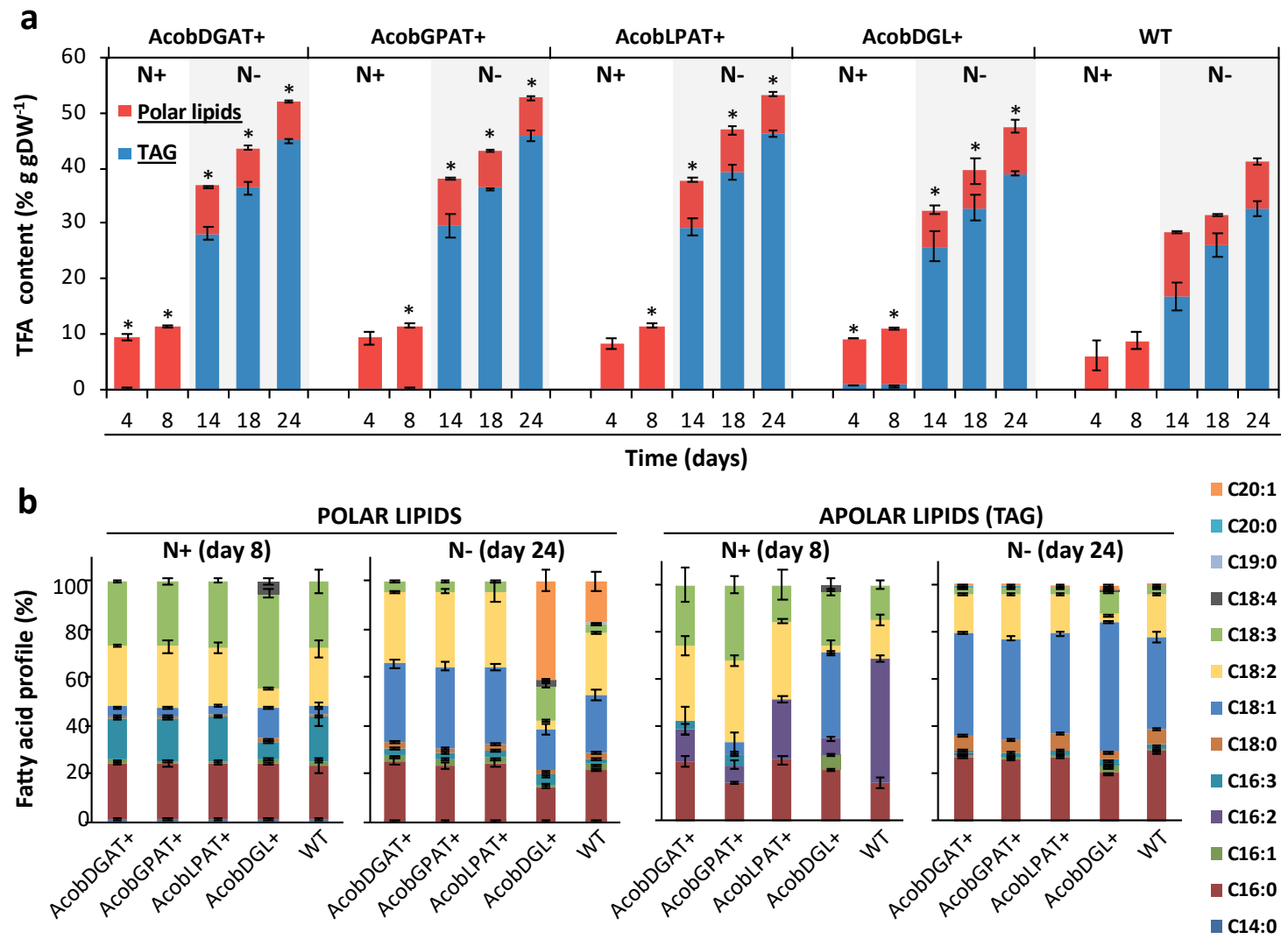

Figure 4. Total fatty acid (TFA), triacylglycerols (TAG) and fatty acid composition in $N$. oleoabundans wild type strain and transformant lines. Total fatty acids: triacylglycerols and polar lipids (a). Polar and apolar lipid (TAG) composition at days 8 and 24 during nitrogen replete $(\mathrm{N}+)$ and depleted $(\mathrm{N}-)$ nitrogen conditions, respectively $(\mathbf{b})$. The data represent the average of $n=3$ replicate experiments. Standard deviation bars are shown. Significant difference between wild type strain and transformant lines is indicated at $P<0.05\left(^{*}\right)$.

\section{Impact of single and multi-gene expression on biomass and fatty acid productivities}

In order to determine performance of all the tested lines we calculated the volumetric biomass, total fatty acid and triacylglycerol productivities. As shown in table 1, during nitrogen replete conditions, $N$. oleoabundans wild type showed high volumetric biomass productivity $\left(413 \mathrm{mg} \cdot \mathrm{L}^{-1} \mathrm{~d}^{-1}\right)$ and growth rate 
$\left(1.23 \mathrm{~d}^{-1}\right)$. The most affected in both volumetric biomass productivity $(231 \mathrm{mg}$ - $\left.\mathrm{L}^{-1} \mathrm{~d}^{-1}\right)$ and growth rate $\left(0.59 \mathrm{~d}^{-1}\right)$ was the three-gene expressing line AcobDGL+. Under nitrogen-depleted conditions, AcobDGAT+, AcobGPAT+, AcobLPAT+ and wild type reached higher volumetric biomass productivities compared to AcobDGL+. The highest volumetric TFA and TAG productivities were obtained during nitrogen-depleted conditions for all the lines. However, remarkably, AcobGPAT+ and AcobDGL+ showed higher volumetric TAG productivity in replete conditions, which were 1 and 4.5-fold higher than wild type, respectively. Under deplete conditions, all the lines expressing single genes showed high TFA and TAG productivities compared to AcobDGL+ and wild type, with AcobLPAT+ reaching the highest values (248.0 and $235 \mathrm{mg} \cdot \mathrm{L}^{-}$ ${ }^{1} \mathrm{~d}^{-1}$, respectively).

Table 1. Growth rate and average volumetric productivities of biomass, total fatty acids (TFA) and triacylglycerols (TAG) for nitrogen replete $(\mathrm{N}+)$ and nitrogen deplete $(\mathrm{N}-)$ conditions. The data represent the average of $n=3$ replicate experiments.

\begin{tabular}{lcccc}
\hline \multicolumn{1}{c}{ Strains } & $\begin{array}{c}\text { Volumetric } \\
\text { biomass } \\
\text { productivity } \\
\left(\mathbf{m g} \cdot \mathbf{L}^{-1} \mathbf{d}^{-\mathbf{1}}\right)\end{array}$ & $\begin{array}{c}\text { Volumetric } \\
\text { TFA } \\
\text { productivity } \\
\left(\mathbf{m g} \cdot \mathbf{L}^{-1} \mathbf{d}^{-1}\right)\end{array}$ & $\begin{array}{c}\text { Volumetric } \\
\text { TAG } \\
\text { productivity } \\
\left(\mathbf{m g} \cdot \mathbf{L}^{-1} \mathbf{d}^{-1}\right)\end{array}$ & $\begin{array}{c}\text { Growth rate } \\
\left(\mathbf{d}^{-1}\right)\end{array}$ \\
\hline WT N+ & $413.1 \pm 50$ & $46.7 \pm 8$ & $0.6 \pm 0.1$ & $1.23 \pm 0.01$ \\
\hline WT N- & $386.5 \pm 35$ & $172.6 \pm 4$ & $192.4 \pm 21$ & - \\
\hline AcobDGAT+ N+ & $254.1 \pm 12$ & $34.9 \pm 6$ & $0.4 \pm 0.1$ & $1.01 \pm 0.01$ \\
\hline AcobDGAT+ N- & $383.1 \pm 26$ & $212.9 \pm 31$ & $202.5 \pm 38$ & - \\
\hline AcobGPAT+ N+ & $321.7 \pm 25$ & $46.1 \pm 8$ & $1.3 \pm 0.2$ & $1.04 \pm 0.01$ \\
\hline AcobGPAT+ N- & $422.2 \pm 44$ & $230.2 \pm 20$ & $224.2 \pm 32$ & - \\
\hline AcobLPAT+ N+ & $324.4 \pm 36$ & $46.4 \pm 8$ & $0.5 \pm 0.1$ & $1.05 \pm 0.01$ \\
\hline AcobLPAT+ N- & $426.5 \pm 40$ & $248.0 \pm 26$ & $235.3 \pm 42$ & - \\
\hline AcobDGL+ N+ & $231.8 \pm 23$ & $33.5 \pm 6$ & $2.7 \pm 0.5$ & $0.59 \pm 0.01$ \\
\hline AcobDGL+ N- & $307.9 \pm 33$ & $167.0 \pm 42$ & $142.5 \pm 47$ & - \\
\hline
\end{tabular}

\section{Discussion}

The oleaginous microalga Neochloris oleoabundans has been previously shown to be an industrially relevant strain for biofuel production due to its ability to 
produce large amounts of lipids, mainly in the form of TAGs, during stress conditions (i.e. nitrogen deprivation) (de Jaeger et al., 2018; Yanqun Li et al., 2008). With the recent advances in biotechnology, tools and techniques for the genetic modification of microalgae are expanding quickly and they could be used for the generation of improved strains with enhanced lipid productivities. It has been demonstrated that overexpression of genes involved in the Kennedy pathway in microalgae species such as Chlamydomonas reinhardtii, Phaeodactylum tricornutum and Nannochloropsis oceanica can lead to higher lipids content. Overexpression of the endogenous LPAT encoding gene in $C$. reinhardtii resulted in an increase of $20 \%$ in total fatty acids (Yamaoka et al., 2016). Similarly, the overexpression of endogenous GPAT encoding gene in $P$. tricornutum led to an enhanced neutral lipid content (2-fold higher than wild type) and specifically, to a significant increase in unsaturated fatty acid profile (Y. F. Niu et al., 2016). Moreover, the diacylglycerol acyltransferase (DGAT) enzyme, responsible for the last step in TAG biosynthesis, has been regarded as a promising target for improving TAG content in multiple studies. Deng et al., (2012)(X.-D. Deng et al., 2012), previously showed that overexpression of the endogenous DGAT encoding gene in C. reinhardtii can result in an increase of total fatty acids by $48 \%$ and $43 \%$ of its dry weight when grown under nitrogen replete and deplete conditions, respectively.

In our study, we report the expression of LPAT, GPAT and DGAT encoding genes from the microalga Acutodesmus obliquus in $N$. oleoabundans. Our results demonstrate that the single expression of LPAT, GPAT and DGAT encoding genes lead to higher lipid accumulation per dry weight during deplete conditions compared to wild type, mainly due to a $~ 1.3$-fold increase in their TAG content. The lipid composition of the apolar fraction remains unchanged compared to wild type, being oleic acid (C18:1), palmitic acid (C16:0) and linoleic acid (C18:2) as the main constituents. Although single gene expression did not lead to higher lipid content during nitrogen replete conditions, we observed a different fatty acid distribution in the apolar fraction. In particular, hexadecadienoic acid (C16:2) decreased and linoleic acid (C18:2) increased substantially compared to wild type. The single expression of LPAT, GPAT and DGAT genes showed no changes in polar lipid distribution during nitrogen 
replete conditions. However, during nitrogen depleted conditions all the three transformant lines seem to favor the production of oleic acid (C18:1) at the expenses of eicosenoic acid (C20:1), which diminished, compared to wild type.

In our attempt to increase the lipid content in $N$. oleoabundans even further, we overexpressed the three LPAT, GPAT and DGAT encoding genes simultaneously. Unexpectedly, in this case we saw an increase in the total fatty acids content of a factor of 1.2-folds compared to wild type, which was lower compared to what we have observed for the single expressing lines. Also, in this case, the major increase was observed in the TAG fraction (figure 4a). In particular, the apolar fraction (TAG) of the multiple gene expressing line contained an increase in $\mathrm{C} 18: 1$ and $\mathrm{C} 18: 3$ fatty acids, and a reduction in C18:0, $\mathrm{C} 16: 0$ and $\mathrm{C} 18: 2$, compared to the single expressing lines and wild type apolar lipid distribution. This suggests that the orchestrated expression of these enzymes shifts the lipid composition towards long unsaturated fatty acid chains. Oleic acid (C18:1) is the major fatty acid produced by $N$. oleoabudans and $A$. obliquus. Moreover, in $A$. obliquus fatty acid profile linolenic acid (C18:3) has higher relative abundance compared to C18:2 (Breuer et al., 2013). Taken together, these findings may suggest a preference of $A$. obliquus enzymes for both $\mathrm{C} 18: 1$ and $\mathrm{C} 18: 3$ fatty acids to be integrated in the triacylglycerols of $N$. oleoabundans. It also suggests that these enzymes are interfering with other lipid metabolic pathway, such as fatty acid elongation and desaturation. Furthermore, also during nitrogen replete conditions the apolar fraction showed an increase in oleic and linolenic fatty acids. Interestingly, the simultaneous gene expression led to substantial changes in the fatty acid profile of the polar fraction. Since the main component of cellular membranes are polar lipids, we hypothesize that the alteration in the lipid distribution might also be correlated to the significant increase in cell size of the multiple gene expressing line. The Kennedy pathway enzymes share precursor like PA and DAG with the enzymes for the biosynthethic pathway of the membrane lipids, such as phosphoglycerides and glycosylglycerides. Further investigations could be focused in understanding which lipid species and how the intermediate precursor pools are affected by the enhanced expression of a heterologous Kennedy pathway, which could elucidate the lipid metabolism in microalgae. 
In order to determine whether overexpression of LPAT, GPAT and DGAT genes had an effect on biomass and fatty acid productivities we calculated the volumetric productivities of all transformants. The highest volumetric TFA and TAG productivities were obtained during nitrogen-starved conditions by single gene expressing lines. Moreover, the expression of single genes led to the highest TFA and TAG productivities, compared to the multiple gene expression and wild type. Previous studies reported that the heterologous expression of DGAT in Scenedesmus obliquus and Phaeodactylum tricornutum increased lipid content and lipid productivities (C. Y. Chen et al., 2016; Zulu et al., 2017). The dual overexpression of the endogenous LPAT and GPAT in P. tricornutum and the multiple expression of the heterologous LPAT, GPAT, PAP and DGAT in Chlorella minutissima showed an enhanced lipid content and productivities as well (Hsieh et al., 2012; X. Wang, Dong, et al., 2018a). Previous studies have reported no effect on growth when overexpressing genes involved in the Kennedy pathway. In our study, the highest volumetric biomass productivity and highest growth rate were achieved by $N$. oleoabundans wild type during nitrogen replete conditions. During nitrogen deplete conditions the specific growth rate was similar among the single overexpressing lines and wild type, whilst the transformant line expressing the three genes simultaneously was the most affected, with a growth rate reduced by half, compared to the replete conditions. Conversely, Wang et al., (2018)(X. Wang et al., 2018) recently reported that the dual expression of GPAT and LPAT in P. tricornutum enhanced growth rate during the mid-log phase, however this was not observed in our transformant line (figure $\mathbf{2 b}$ ).

In previous reports, it has been shown that the overexpression of these single enzymes did not affect the photosynthetic efficiency of the transformant lines. Thus, it has been hypothesized that the increased availability of fatty acid precursors for TAG synthesis, such as phosphatidic acid (PA), induced by the overexpression of these enzymes, may also serve as precursors for the synthesis of plastidic membranes, resulting in elevated photosynthetic efficiencies (Balamurugan et al., 2017; Y. F. Niu et al., 2013, 2016). In our study, we did not observe a significant change in photosynthetic efficiencies in the lines overexpressing single genes, compared to wild type. On the other 
hand, the multiple gene expression showed a significantly reduced photosynthetic activity, indicating that the introduction of these three genes results in an increased metabolic load that has a direct impact on the physiological status of the cells. In this regard, the reduced quantum yield and size enlargement in this transformant line may also be related to a stress component, which could be correlated to the alteration of both polar and apolar lipid compositions, interestingly different compared to wild type and the single gene expressing lines.

We further investigated the biochemical composition of all transformant lines in order to determine the effect of gene expression on the synthesis of cellular components. Our results showed that only when the three genes were overexpressed the carbohydrate content was reduced at the end of the experiment. Moreover, all the transformant lines showed a reduced protein content during nitrogen depleted conditions. Similarly, Balamurugan et al., (2017)(Balamurugan et al., 2017) and Wang et al., (2018)(X. Wang et al., 2018) reported that overexpression of GPAT or dual expression of GPAT and LPAT in P. tricornutum, respectively, led to lower protein and carbohydrate content in starved conditions. These results suggest that carbohydrate and protein metabolism are redirected towards lipid production, and in particular, the orchestrated expression of the three genes has a stronger impact in redirecting the carbon flux from carbohydrates to lipids. On the other hand, during nitrogen replete experiments we observed higher protein content compared to the wild type strain as a result of the high expression of LPAT, GPAT and DGAT genes, which is in accordance with previous studies (Dinamarca et al., 2017). Other cellular components such as chlorophylls and carotenoids were also affected by the gene expression. AcobGPAT+ and AcobLPAT+ showed a reduced chlorophyll a, chlorophyll b and carotenoid content, suggesting that the carbon flux might have been redirected towards lipid production. Contrarily, the transformant line expressing the three genes simultaneously showed higher chlorophyll and carotenoid content, compared to wild type and the single gene expressing lines. Since the multi-gene expressing line showed a detrimental effect in the photosynthetic efficiency, lower protein 
and carbohydrate content, we hypothesize that the higher pigment content in the cells may be induced to prevent photoinhibition and oxidative stress.

\section{Conclusions}

In this study, we report the effect of the heterologous overexpression of LPAT, GPAT and DGAT genes in N. oleoabundans. Single and multiple gene expression resulted in an enhanced lipid production for all the transformant lines. We demonstrated that higher neutral lipid content and lipid productivities were achieved when single genes were expressed during nitrogen depleted conditions. Moreover, the single gene expression did not affect growth, biomass productivities and photosynthetic activities significantly. Interestingly, the simultaneous expression of LPAT, GPAT and DGAT had a negative effect on growth and photosynthetic activity, which resulted in 4-fold bigger cells, lower biomass and lipid productivities. However, during replete conditions we observed the highest TAG productivity, which was 4.5 -fold higher than wild type. This result indicates that the orchestrated expression of the three genes can induce TAG production during nitrogen replete conditions. However, in order to maximize lipid productivities even further, optimal cultivation conditions and homologous and dual-gene expression need to be investigated in future work. Moreover, future investigations on the alteration of lipid species and precursor pools by the heterologous expression of the Kennedy pathway can help to further elucidate the lipid metabolism in microalgae. Overall, our results provide insights regarding the Kennedy pathway related lipid production and composition, and the improvement of TAG production in microalgae. This approach could lead to the generation of microalgal strains with economical potential for the successful biofuel production.

\section{Authors Contributions}

CFM designed the experiment. CFM performed the experiments, analyzed, interpreted the data and wrote the manuscript. SD, RAW and RHW supervised 
the project. All authors contributed to the work, discussed the results, read and approved the final version of this manuscript.

\section{Conflict of Interest Statement}

The authors declare that there are no conflicts of interest related to this work.

\section{Funding}

This research project was funded by the National Commission of Scientific and Technologic Research of Chile (CONICYT).

\section{List of abbreviations}

TAG, triacylglycerol

TFA, Total fatty acids

LPAT, Lysophosphatidic acid acyltransferase

GPAT, Glycerol -3-phophate acyltransferase

DGAT, Diacylglycerol acyltransferase

ER, Endoplasmic reticulum

GFP, Green fluorescent protein

PCR, Polymerase chain reaction

FW, Fresh water

DW, Dry weight

WT, Wild type 


\section{Acknowledgements}

This research project was financially supported by the National Commission of Scientific and Technologic Research of Chile (CONICYT). 



\section{Chapter 5}

\section{Genetic engineering of microalgae for enhanced lipid production}

This chapter has been published as: Muñoz, C.F., Südfeld, C., Naduthodi, M.I.S., Weusthuis, R.A., Barbosa, M.J., Wijffels, R.H. D'Adamo, S. (2021). Genetic engineering of microalgae for enhanced lipid production. Biotechnology Advances, 52: 107836. 


\section{Abstract}

Microalgae have the potential to become microbial cell factories for lipid production. Their ability to convert sunlight and $\mathrm{CO}_{2}$ into valuable lipid compounds has attracted interest from cosmetic, biofuel, food and feed industries. In order to make microalgae-derived products cost-effective and commercially competitive, enhanced growth rates and lipid productivities are needed, which require optimization of cultivation systems and strain improvement. Advances in genetic tool development and omics technologies have increased our understanding of lipid metabolism, which has opened up possibilities for targeted metabolic engineering. In this review we provide a comprehensive overview on the developments made to genetically engineer microalgal strains over the last 30 years. We focus on the strategies that lead to an increased lipid content and altered fatty acid profile. These include the genetic engineering of the fatty acid synthesis pathway, Kennedy pathway, polyunsaturated fatty acid and triacylglycerol metabolisms and fatty acid catabolism. Moreover, genetic engineering of specific transcription factors, NADPH generation and central carbon metabolism, which lead to increase of lipid accumulation are also reviewed.

\section{Keywords}

Microalgae, fatty acids, triacylglycerols (TAG), polyunsaturated fatty acids (PUFA), genetic engineering, omics, gene editing, overexpression, heterologous expression

\section{Abbreviations}

ACBP: acyl-CoA-binding protein; ACC: acetyl-CoA carboxylase; ACP: acyl carrier protein; ACS: acetyl-COA synthetase; ALA: a-linolenic acid; ALE: adaptive laboratory evolution; AP2-type TF: Apetala 2-type transcription factor; ARA: arachidonic acid; ATMT: Agrobacterium tumefaciens-mediated transformation; ATPase: ATP synthase; BCR: breakpoint cluster region protein; BCR1: biotin carboxylase; bHLH: basic helix-loop-helix; bZIP: basic leucine zipper; CAO: chlorophyllide a oxygenase; CBB cycle: Calvin-BensonBassham cycle; CBP-like protein SN03: SN = chitin binding protein-like protein stress- 
nitrogen 03; CHT7: Compromised Hydrolysis of TAG 7 (protein); DAG: diacylglycerol; DAGK: diacylglycerol kinase; DGAT: diacylglycerol acyltransferase; DGTS: diacylglyceroltrimethylhomoserine; DGTT1: type 2 diacylglycerol acyltransferase; DHA: docosahexaenoic acid; DHAP: dihydroxyacetone phosphate; DOF-type TF: DNA-binding with one finger type transcription factor; DPA: docosapentaenoic acid; ELO: elongase; EMS: ethyl methanesulfonate; ENR: enoyl-ACP reductase; EPA: eicosapentaenoic acid; F2BP: fructose 2,6 bisphosphatase; FA: fatty acids; FAD: fatty acid desaturase; FAS: fatty acid synthesis; FAT1: acyl carrier protein thioesterase; FNR: ferredoxin NADP+ oxidoreductase; G3P: glycerol 3-phosphate; G3PDH: glyceraldehyde 3-phosphate dehydrogenase; G6P: glucose 6-phosphate; G6PD: glucose 6-phosphate dehydrogenase; gdcw: gram dry cell weight; GK: glycerol kinase; GPAT: glycerol 3-phosphate acyltransferase; GPAT2: glycerol-3-phosphate acyltransferase 2; HD: 3-hydroxyacyl-ACP dehydrase; HE: heterologous expression; HES: heterologous expression strain; IDH: isocitrate dehydrogenase; KAR: 3-ketoacyl-ACP reductase; KAS: 3-ketoacyl-ACP synthase; KD: knockdown; KDS: knockdown strain; KO: knockout; KOS: knockout strain; LA: linoleic acid; LC-FACS: long-chain fatty acyl-CoA synthetase; LC-PUFA: long-chain polyunsaturated fatty acid; LD: lipid droplets; LPAT and LPAAT: Iysophophatidic acid acyltransferase; MAGL: monoacylglycerol; MAT: malonyl-CoA ACP transacylase; MAT: malonyl-CoA: ACP transacylase; MCFA: medium-chain fatty acid; ME: malic enzyme; MGDG: monogalactosyldiacylglycerol; MUFA: monounsaturated fatty acid; NTG: N'-nitro$\mathrm{N}$-nitrosoguanidine; OA: oleic acid; OE: overexpression; OES: overexpression strain; oxPPP: oxidative pentose phosphate pathway; PA: phosphatidic acid; PAP: phosphatidic acid phosphatase; PC: phosphatidylcholine; PDAT: phospholipid diacylglycerol acyl transferase; PE: phosphatidylethanolamine; PFK2: 6-phosphofructo-2-kinase; PI: phosphatidylinositol; PNPLA3: Patatin-like phospholipase domain-containing protein 3; PPDK: pyruvate phosphate dikinase; PS: parental strain; PSI: photosystem I; PSII: photosystem II; PSR1: phosphorus starvation response 1 (protein); PUFA: polyunsaturated fatty acids; RNAi: RNA interference; ROS: reactive oxygen species; SFA: saturated fatty acid; SFA: saturated fatty acid; SOD1: superoxide dismutase; SQD1: UDP-sulfoquinovose synthase; StLDP: Stramenopile lineage-specific lipid droplet protein; TAG: triacylglycerol; TAGL: triacylglycerol lipase; TE: thioesterase; TF: transcription factor; TFA: total fatty acids; UGDH: UDP-glucose 6-dehydrogenase; WT: wild-type 


\section{Introduction}

Microalgae are a large polyphyletic group of unicellular eukaryotic photosynthetic microorganisms that are considered as promising platforms for sustainable production of bioproducts due to their ability to convert solar energy and carbon dioxide into organic compounds of commercial interest. Their role in carbon-sequestration and their ability to grow on freshwater, seawater or wastewater at high growth rate present the prospect to mitigate environmental issues and to reduce greenhouse gas emissions. Moreover, microalgae cultivation does not compete with agricultural food production, as it does not require arable land. The vast range of bioactive compounds naturally present in microalgal biomass, including antioxidants, carotenoids, proteins, polysaccharides, polyunsaturated fatty acids (PUFAs), triacylglycerols (TAGs), sterols and vitamins has attracted the attention of biotechnological, pharmaceutical, cosmetic, biofuel, food and feed industries (Chisti, 2007; Christaki et al., 2011; Draaisma et al., 2013; Pulz \& Gross, 2004).

Therefore, microalgae have become an important subject of study in the last 50 years. During 1978-1996 the U.S Department of Energy's Aquatic Species Program analyzed over 3000 microalgae species for their potential use for biofuel production (Sheehan et al., 1998). Extensive research and development were conducted to identify species with desirable traits including high lipid content and productivity, competitiveness in outdoor cultivation and tolerance to fluctuations in temperature and salinity. Furthermore, evaluation and optimization of large-scale cultivation systems, harvesting, extraction and refinery techniques were performed. Despite the twenty-year research period, the Aquatic Species Program and Biofuels Program did not yield any strain with suitable characteristics for cost-effective large-scale lipid production. Nevertheless, bioprospecting continues and researchers are characterizing new microalgae species in terms of growth and lipid composition (Barkia et al., 2019; Breuer et al., 2012; Duong et al., 2015; Ferreira et al., 2019; Gim et al., 2014; Lim et al., 2012). 
Oleaginous microalgal species belonging to genera Acutodesmus, Phaeodactylum, Dunaliella and Nannochloropsis have attracted interest in the last two decades (Fu et al., 2019). When exposed to nutrient limitation or starvation, these species can accumulate neutral lipids in the form of TAGs, which can be converted into renewable fuels of third generation via transesterification (Chisti, 2007; Wijffels \& Barbosa, 2010; Aratboni et al., 2019). Microalgal lipids are, furthermore, receiving growing interest from the food industry as an edible plant and fish oil substitute and as an alternative source of PUFAs such as omega- 3 and omega- 6 fatty acids, which provide health benefits when used as dietary supplement (Ghiffary et al., 2019).

Despite the industrial relevance and environmental benefits of microalgae compared to land plants, commercial-scale production of microalgae-derived commodity chemicals is not yet economically feasible due to high production costs. Recent studies have estimated that the production of one kilogram of biomass containing $24 \%$ of TAG in a 100 ha facility located in the south of Spain would cost $3.4 € \mathrm{~kg}^{-1}$ of biomass using flat panel photobioreactors (Ruiz et al., 2016). This study indicates the potential of implementing microalgae as efficient microbial cell factories for lipid production at large scale, using stateof-the-art cultivation systems. Improving microalgal strains could contribute to cost reduction and bring industrial production of microalgal lipids within reach. Remmers et al. (2018) have determined that the maximum theoretical lipid yield is about five times higher than what is currently achieved in outdoor settings, clearly indicating room for improvement (Benvenuti et al., 2016; Remmers et al., 2018; Ruiz et al., 2016). Advances in research fields such as synthetic biology and genetic engineering can complement current efforts to achieve an economically feasible process.

Strain improvement via direct or indirect genetic modification has been proposed as a methodology to enhance growth and lipid productivities in promising microalgal strains (Radakovits et al., 2010). Wild type microalgal strains accumulate lipids mainly under nutrient-limited conditions, which limits biomass productivities. Genetic engineering is a potential strategy to generate strains that accumulate lipids without growth impairment. This review provides 
an overview of genetic engineering strategies implemented in microalgae for improving the lipid content and fatty acid profile over the last 30 years.

\subsection{Genetic tool development and metabolic approach}

In the last decades, considerable progress has been made in the development of genetic tools and generation of genetically improved strains (Korkhovoy et al., 2016; Kumari et al., 2015; Lin et al., 2019). Genetic modification has been achieved by adaptive laboratory evolution (ALE), random mutagenesis and direct genetic engineering. ALE and physical mutagens such as UV light, gamma radiation and $\mathrm{X}$-rays as well as chemical mutagens such as $\mathrm{N}^{\prime}$-nitro- $\mathrm{N}$ nitrosoguanidine (NTG) and ethyl methanesulfonate (EMS) have been successfully applied on microalgae for introduction of random mutations. However, efficient genetic engineering strategies that could generate specific insertions, deletions or substitutions in the host genome are required to make targeted modifications while avoiding unpredictable results. Advances in sequencing technology, development of fast, accurate and efficient DNA delivery systems and development of high throughput genome editing tools have become a crucial component for the generation of genetically improved microalgal strains.

The first major breakthrough in microalgal biotechnology was the nuclear transformation of Chlamydomonas reinhardtii in 1990 (figure 1). Kindle (1990) performed agitation of cells in the presence of glass beads coated with DNA which allowed the creation of micro-pores in the cell membrane due to friction, causing the passage of DNA molecules into the cell. Although this development was made three decades ago, the DNA delivery into microalgal cells is still a major bottleneck due to the variation in cell sizes, cell wall structures and composition among the several genera of microalgae. Several transformation methods have been developed for the delivery of exogenous DNA into different microalgal species. The most common and successful techniques are electroporation, Agrobacterium tumefaciens-mediated transformation, particle bombardment and agitation with glass beads (Apt et al., 1996; Chow \& Tung, 1999; T. G. Dunahay, 1993; Economou et al., 2014; 
Guo et al., 2013; K. L. Kindle, 1990; Karen L. Kindle, 1998; Ramesh et al., 2011; Shimogawara et al., 1998; Suttangkakul et al., 2019; C. Tan et al., 2005). Most of these methods are restricted to a limited number of species and require laborious optimization when applied to other microalgae. Moreover, most of the transformation methods can lead to high cell death because harsh treatments are required to overcome recalcitrant microalgal cell barriers (Muñoz et al., 2018).

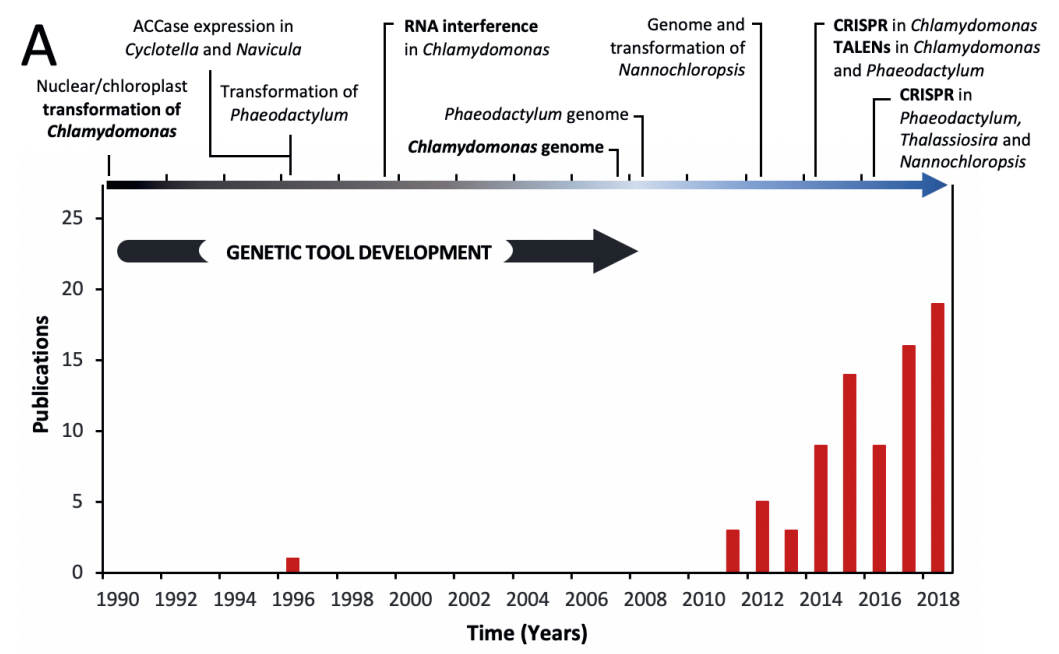

\begin{tabular}{lc|}
\hline B & \\
\hline Genetic modification & Publications \\
\hline Kennedy pathway & 31 \\
Transcription factors & 12 \\
NADPH generation & 12 \\
PUFAS metabolism & 10 \\
FAS metabolism & 14 \\
Central carbon metabolism & 3 \\
Others & 5 \\
\hline Total & 87 \\
\hline
\end{tabular}

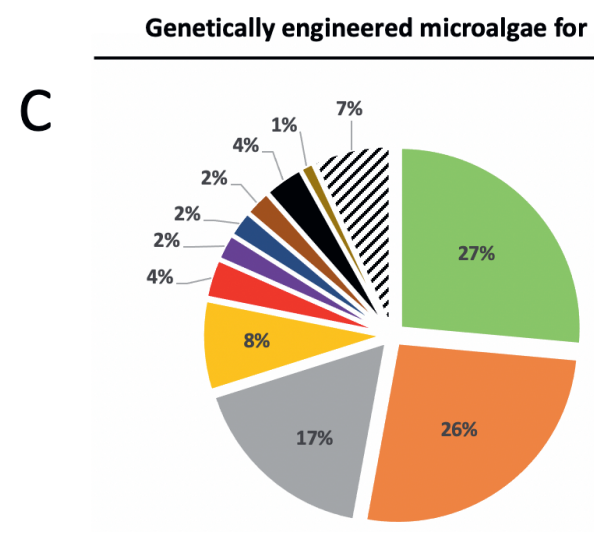

Figure 1. Overview of genetic engineering studies for enhancing lipid production in microalgae. (A) Timeline of genetic tool development (top $x$-axis) and number of publications describing targeted genetic engineering of microalgae to enhance lipid production (bottom x-axis) (Apt et al., 1996; Bowler et al., 2008; Daboussi et al., 2014; Dunahay et al., 1996; Gao et al., 2014; Hopes et al., 2016; Jiang et al., 2014; Kindle, 1990; Kindle et al., 1989; Merchant et al., 2007; Nymark et al., 2016; Radakovits et al., 2012; Schroda et al., 1999; Wang et al., 2016). (B) Publications presented in this review. (C) Frequency of engineered microalga species. 
Recent advances in omics technology and the development of genome scale metabolic models have increased our understanding of microalgal metabolism (figure 1). However, the lack of data available on genome sequencing and functional annotation for microalgal species limits the possibility of exploiting omics technologies (Lauritano et al., 2019; W. R. Lin et al., 2019; Y. T. Zhang et al., 2019). While genome-scale metabolic models are powerful tools for predicting metabolic engineering strategies, they could be further improved by experimental characterization of enzymes, kinetic analyses, and identification of gene regulation patterns. An increase in genetic data availability and the validation of metabolic models will allow effective and strategic design of genetic approaches and will improve the accuracy and reliability of bioinformatic tools.

Although omics data for microalgae are in a developing stage, they have allowed the identification of regulatory elements necessary for the development of genetic engineering strategies. Identification of promoters, splicing signals, terminators, selection markers and reporter genes have facilitated the expression of heterologous genes in microalgae. This has accelerated the development of molecular tools including state-of-the-art genome editing systems such as Clustered Regularly Inter-Spaced Palindromic Repeats and associated proteins (CRISPR-Cas), Transcription Activator-Like Effector Nuclease (TALEN), Zinc Finger Nulease (ZFN) and RNA interference (RNAi). Most of these genetic tools have been used throughout the last decades in several publications (Kumari et al., 2015; Lin et al., 2019). Figure 1 summarizes the collective efforts and advancements made in the last 30 years on genetic engineering of microalgae, including tool development and strategies aimed at enhancing lipid production. Recent research has demonstrated that this goal can be achieved by tuning fatty acid synthesis (FAS), Kennedy pathway, PUFA and TAG metabolism, transcription factors and nicotinamide adenine dinucleotide phosphate (NADPH) generation. Nevertheless, most of the studies focused on the genetic modification of model microalgal strains such as Chlamydomonas reinhardtii, Phaeodactylum tricornutum and more recently Nannochloropsis spp., due to the lack of a universal genetic toolbox for all microalgal species (figure 1). 


\subsection{Fatty acid synthesis (FAS) metabolism}

Fatty acid synthesis (FAS) has been a logical target for improving the lipid production as it is the first step towards de novo lipid formation in microalgae. FAS occurs in the chloroplast with acetyl-CoA as the main precursor, which is converted to malonyl-CoA by an acetyl-CoA carboxylase (ACC) enzyme. Malonyl-CoA is then converted by malonyl-CoA ACP transacylase (MAT) to form malonyl-ACP by transferring a malonyl-CoA to the acyl carrier protein (ACP). Subsequently, malonyl-ACP undergoes a series of repeated rounds of condensation, reduction, dehydration and again reduction steps. These reactions are catalyzed by the enzymes 3-ketoacyl-ACP synthase (KAS), 3ketoacyl-ACP reductase (KAR), 3-hydroxyacyl-ACP dehydrase (HD), and enoylACP reductase (ENR) to finally form saturated fatty acids (SFA) such as C14:0, C16:0 and C18:0, whose length is determined by specific acyl-acyl carrier protein thioesterases (TEs) (figure 2).

Malonyl-CoA formation is catalyzed by acetyl-CoA carboxylase, and it is the major rate-limiting step for the de novo biosynthesis of fatty acids (Li-Beisson et al., 2019). Therefore, microalgal ACC genes have been overexpressed in multiple species, albeit with limited effect on lipid accumulation. The first attempt in this line of research was made by Dunahay et al. (1996) by overexpressing the native ACC protein in the microalgae Cyclotella cryptica and Navicula saprophila. Although they showed that overexpression resulted in a significant increase in ACC activity, no differences were observed regarding intracellular lipid content. On the other hand, overexpression of a yeast-derived ACC gene in Scenedesmus quadricauda resulted in 1.6-fold increase in total fatty acids (Gomma et al., 2015).

Fatty acid biosynthesis requires a continuous supply of acetyl-CoA. One of the main enzymes responsible for increasing intracellular acetyl-CoA pools is the acetyl-CoA synthetase (ACS). This enzyme catalyzes the conversion of acetate into acetyl-CoA and it has been a promising target for genetic engineering. An Escherichia coli acetyl-CoA synthetase was overexpressed in the marine microalga Schizochytrium sp., resulting in $11.3 \%$ increase in fatty acids and 
29.9\% increase in biomass content, improved carbon utilization and reduced intracellular acetate concentration (J. Yan et al., 2013). Moreover, overexpression of ACS in C. reinhardtii has increased intracellular acyl-CoA pools leading to 2.4-fold increase in TAG content when mutants were grown under nitrogen starvation conditions (Rengel et al., 2018).

Other recent studies have met with success by overexpressing the enzyme malonyl-CoA ACP transacylase (MAT) in N. oceanica and Schizochytrium sp., increasing the total lipid content by 36 and $10.1 \%$ compared to wild type strains, respectively (J.-W. Chen et al., 2017; Z. Li et al., 2018). Chen et al. (2017) demonstrated that neutral lipid contents in mutant strains of Nannochloropsis was increased by $31 \%$ and they reported higher growth rates and photosynthetic efficiencies. Li et al. (2018) reported an increase of $24.5 \%$ in PUFA production of transgenic Schizochytrium strains, increasing DHA and EPA yields by 172.5 and $81.5 \%$, respectively.

The mature fatty acid assembled on the ACP is hydrolyzed by an acyl-acyl carrier protein thioesterase, which determines the length and type of the fatty acid produced. Although microalgal TEs remain largely uncharacterized, studies suggest that these enzymes are distinct from their counterparts in plants. Plants have developed TEs for different acyl chain lengths (FatA and FatB), while microalgae use one thioesterase with broad specificity, Fat1 (Blatti et al., 2012). Several studies have attempted to express endogenous or exogenous TEs to manipulate length and type of fatty acids and to synthesize more saturated, medium-chain fatty acids (MCFAs, C8-14), which are preferable for biodiesel and other bulk chemical applications (Gong et al., 2011; K. W. M. Tan \& Lee, 2017; Q. Wang et al., 2021). In particular, a C12:0-biased FatB TE from Umbellularia californica and myristic acid (C14:0)-biased FatB TE from Cinnamomum camphora were used to redirect FA synthesis in the microalga $P$. tricornutum, obtaining a composition of $5 \% \mathrm{w} / \mathrm{w} \mathrm{C12:0}$ and $12 \% \mathrm{w} / \mathrm{w} \mathrm{C} 14: 0$ of TFAs, respectively (Radakovits et al., 2011). By using a C12-specific TE and an MCFA-specific KAS, it was possible to increase the accumulation of $\mathrm{C} 12: 0$ and C14:0 in Dunaliella tertiolecta up to 0.4 and $1.4 \% \mathrm{w} / \mathrm{w}$ of TFAs, respectively (H. Lin et al., 2018; H. Lin \& Lee, 2017). 
Table 1. Genetic engineering of FAS metabolism for enhanced lipid production.

\begin{tabular}{|c|c|c|c|c|c|}
\hline Algal Strain & $\begin{array}{l}\text { Targeted } \\
\text { Genes }\end{array}$ & Strategy & $\begin{array}{l}\text { Effect on lipid } \\
\text { synthesis }\end{array}$ & Comments & References \\
\hline $\begin{array}{l}\text { C. cryptica and } N \text {. } \\
\text { saprophila }\end{array}$ & ACCase & $\mathrm{OE}, \mathrm{HE}$ & $\begin{array}{l}\text { No effect on lipid } \\
\text { content }\end{array}$ & $\begin{array}{l}\text { Significantly increased } \\
\text { ACCase activity }\end{array}$ & $\begin{array}{l}\text { (Terri G. } \\
\text { Dunahay et } \\
\text { al., 1996) }\end{array}$ \\
\hline S. quadricauda & $\begin{array}{l}\text { ACC1 } \\
\text { GPD1 } \\
\text { GUT1 }\end{array}$ & $\mathrm{HE}$ & $\begin{array}{l}\text { ACC1, GPD1 and } \\
\text { GUT1 increased } \\
\text { lipid content by } 1.6, \\
1.6 \text { and } 1.9 \text {-fold. } \\
\text { Multi-gene } \\
\text { expression } \\
\text { increased lipid } \\
\text { content by } 1.45- \\
\text { fold }\end{array}$ & $\begin{array}{l}\text { No significant effect on } \\
\text { growth among all } \\
\text { transgenic strains }\end{array}$ & $\begin{array}{l}\text { (Gomma et } \\
\text { al., 2015) }\end{array}$ \\
\hline $\begin{array}{l}\text { Schizochytrium } \\
\text { sp. }\end{array}$ & $A C S$ & $\mathrm{HE}$ & $\begin{array}{l}11.3 \% \text { increase in } \\
\text { fatty acids }\end{array}$ & $\begin{array}{lll}29.9 \% & \text { increase } & \text { in } \\
\text { biomass } & & \\
\end{array}$ & $\begin{array}{l}\text { (J. Yan et al., } \\
\text { 2013) }\end{array}$ \\
\hline C. reinhardtii & ACS2 & $\mathrm{OE}$ & $\begin{array}{l}\text { 2.4-fold increased } \\
\text { TAG content under } \\
\mathrm{N} \text {-starvation } \\
\text { conditions }\end{array}$ & $\begin{array}{l}\text { 2-fold increased starch } \\
\text { content and } 60 \% \text { higher } \\
\text { acyl-CoA in } \mathrm{N} \text {-replete } \\
\text { conditions }\end{array}$ & $\begin{array}{l}\text { (Rengel et } \\
\text { al., 2018) }\end{array}$ \\
\hline N. oceanica & NoMCAT & $\mathrm{OE}$ & $\begin{array}{l}36 \% \text { increased TFA } \\
\text { and } 31 \% \text { increased } \\
\text { neutral lipid } \\
\text { content. Increased } \\
\text { C20:5 by } 8 \%\end{array}$ & $\begin{array}{l}\text { Higher growth rate and } \\
\text { photosynthetic } \\
\text { efficiency compared to } \\
\text { wild type strains }\end{array}$ & $\begin{array}{l}\text { (J.-W. Chen } \\
\text { et al., 2017) }\end{array}$ \\
\hline $\begin{array}{l}\text { Schizochytrium } \\
\text { sp. }\end{array}$ & MAT & $\mathrm{OE}$ & $\begin{array}{l}10.1 \text { and } 24.5 \% \\
\text { increased TFA and } \\
\text { PUFA content, } \\
\text { respectively. TFA, } \\
\text { DHA and EPA yields } \\
\text { increased by } 39.6, \\
81.5 \text { and } 172.5 \% \text {, } \\
\text { respectively }\end{array}$ & $\begin{array}{l}\text { Carotene content was } \\
\text { decreased and } \\
\text { redirected toward PUFA } \\
\text { synthesis }\end{array}$ & $\begin{array}{l}\text { (Z. Li et al., } \\
\text { 2018) }\end{array}$ \\
\hline P. tricornutum & $\begin{array}{l}\text { UCFATB } \\
\text { CCFATB }\end{array}$ & $\mathrm{HE}$ & $\begin{array}{l}\text { Increased } \\
\text { accumulation of } \\
\text { C12:0 of up to } \\
6.2 \% \text { of TFA and } \\
\text { C14:0 of up to } \\
15 \% \text {. } 75-90 \% \text { of } \\
\text { the shorter chain } \\
\text { length fatty acids } \\
\text { were incorporated } \\
\text { into TAGs }\end{array}$ & $\begin{array}{l}\text { No significant secretion } \\
\text { of fatty acids was } \\
\text { observed }\end{array}$ & $\begin{array}{l}\text { (Radakovits } \\
\text { et al., 2011) }\end{array}$ \\
\hline P. tricornutum & PtTE & $\mathrm{OE}$ & $\begin{array}{l}\text { Overexpression did } \\
\text { not alter fatty acid } \\
\text { composition. } \\
\text { Enhanced TFA by } \\
72 \%\end{array}$ & $\begin{array}{lr}\text { Overexpression in } & E . \\
\text { coli increased } & \text { TFA } \\
\text { content } & \text { and } \\
\text { composition } & \text { in } \\
\text { membrane lipid } & \\
\end{array}$ & $\begin{array}{l}\text { (Gong et al., } \\
\text { 2011) }\end{array}$ \\
\hline C. reinhardtii & CrTE & $\mathrm{OE}$ & $\begin{array}{l}\text { No significant } \\
\text { change in fatty acid } \\
\text { content. } 2.5 \text {-fold } \\
\text { increased levels of } \\
\text { short-chain fatty } \\
\text { acids (C14:0) }\end{array}$ & $\begin{array}{l}\text { Demonstration of } \\
\text { protein-protein } \\
\text { interactions between } \\
\text { fatty acid acyl carrier } \\
\text { protein (ACP) and } \\
\text { thioesterase (TE) }\end{array}$ & $\begin{array}{l}\text { (Blatti et al., } \\
\text { 2012) }\end{array}$ \\
\hline C. reinhardtii & DtTE & $\mathrm{HE}$ & $\begin{array}{l}63-69 \% \text { increase in } \\
\text { neutral lipids and } \\
56 \% \text { improvement } \\
\text { in TFA }\end{array}$ & $\begin{array}{l}\text { No significant effect on } \\
\text { growth }\end{array}$ & $\begin{array}{l}\text { (K. W. M. } \\
\text { Tan \& Lee, } \\
\text { 2017) }\end{array}$ \\
\hline D. tertiolecta & C14TE & $\mathrm{HE}$ & $\begin{array}{lr}\text { C12:0 r r } & \text { was } \\
\text { increased } & \text { from } \\
0.32 \text { to } 0.43 \% \text { and } \\
\mathrm{C} 14: 0 & \text { was }\end{array}$ & $\begin{array}{l}\text { Medium chain length } \\
\text { fatty acid levels were } \\
\text { negatively correlated } \\
\text { with expression of fatty }\end{array}$ & $\begin{array}{l}\text { (H. Lin et al., } \\
\text { 2018) }\end{array}$ \\
\hline
\end{tabular}


Chapter 5

\begin{tabular}{|c|c|c|c|c|c|}
\hline & & & $\begin{array}{l}\text { increased from } \\
0.75 \text { to } 1.47 \% \mathrm{w} / \mathrm{w} \\
\text { of TFAs }\end{array}$ & $\begin{array}{l}\text { acid synthesis genes; } \\
\text { KASII, } \Delta 9 D \text { and } \Delta 12 D\end{array}$ & \\
\hline C. reinhardtii & CrFAB2 & $\mathrm{OE}$ & $\begin{array}{l}\text { oleic acid (C18:1) } \\
\text { increased by } 2.4- \\
\text { fold compared to } \\
\text { wild-type strain. } \\
\text { TFA increased by } \\
28 \%\end{array}$ & $\begin{array}{l}\text { CrFAB2 overexpression } \\
\text { resulted in induction of } \\
\text { CrFAD2 expression. No } \\
\text { significant effect on } \\
\text { growth }\end{array}$ & $\begin{array}{l}\text { (Hwangbo et } \\
\text { al., 2014) }\end{array}$ \\
\hline C. merolae & $\begin{array}{l}\text { acyl-ACP } \\
\text { reductase }\end{array}$ & $\mathrm{HE}$ & $\begin{array}{l}\text { 3-fold increased } \\
\text { TAG content, which } \\
\text { led to an increase in } \\
\text { the number and } \\
\text { size of lipid droplets }\end{array}$ & $\begin{array}{l}\text { Expression led to up- } \\
\text { regulation of genes } \\
\text { related to degradation } \\
\text { of branched chain } \\
\text { amino acids, fatty acid } \\
\text { synthesis } \\
\begin{array}{ll}\text { degradation. } \\
\text { significant effect ond } \\
\text { growth }\end{array} \\
\end{array}$ & $\begin{array}{l}\text { (Sumiya et } \\
\text { al., 2015) }\end{array}$ \\
\hline P. tricornutum & MaKCS & $\mathrm{HE}$ & $\begin{array}{l}0.2 \% \text { content of } \\
\text { nervonic } \\
(\mathrm{C} 24: 1) \text { in total } \\
\text { FAMEs }\end{array}$ & $\begin{array}{l}\text { No presence of nervonic } \\
\text { acid in wild type strain }\end{array}$ & $\begin{array}{l}\text { (Y. Fan et } \\
\text { al., 2018) }\end{array}$ \\
\hline
\end{tabular}




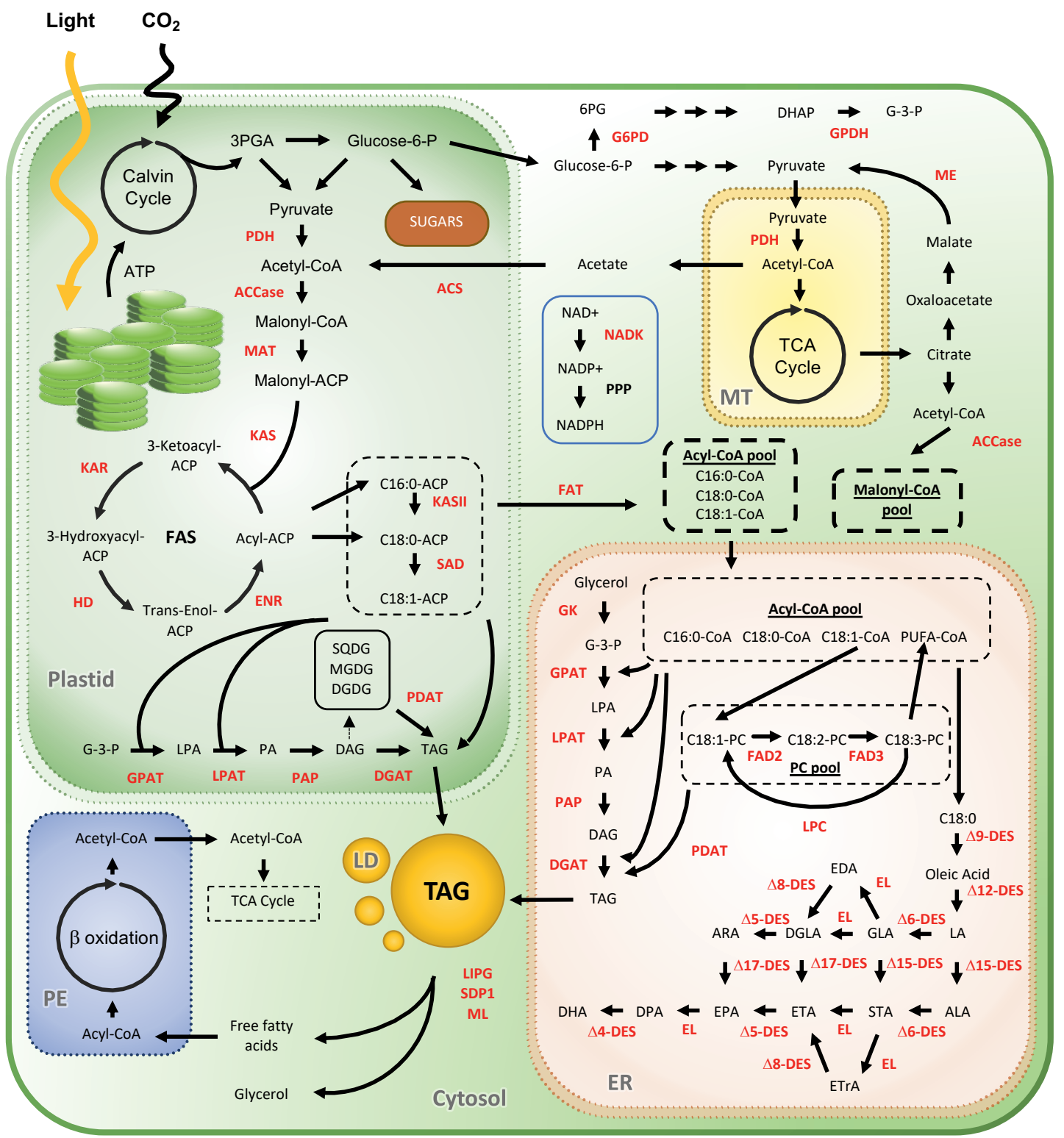

Figure 2. Microalgal lipid biosynthesis pathways. Cellular organelles: plastid; ER, endoplasmic reticulum; PE, peroxisome; MT, mitochondria; LD, lipid droplets.

\subsection{Kennedy pathway}

Microalgae can accumulate large amounts of neutral lipids when exposed to stress conditions such as nutrient limitation (Janssen et al., 2018). These lipids are mainly composed of saturated or monounsaturated fatty acyl groups, which are of great industrial interest for their potential use as a feedstock for biofuel production (Brennan \& Owende, 2010). Among neutral lipids that can be found in microalgal cells, the most common saturated fatty acids are C14:0, C16:0 
and C18:0 (Zulu et al., 2018). Moreover, the most abundant neutral lipids found in microalgae are monoacylglycerols (MAGLs), diacylglycerols (DAGs) and triacylglycerols (TAGs), the latter being a major lipid class in cellular storage compounds such as lipid bodies. Glycerolipids are synthesized in the Kennedy pathway, which begins with glycerol 3-phosphate (G3P). Acylation of glycerol-3-phosphate (G3P) is catalyzed by glycerol 3-phosphate acyltransferase (GPAT) to form lyso-phosphatidic acid, which is further converted into phosphatidic acid by lysophophatidic acid acyltransferase (LPAT). Phosphatidic acid is then dephosphorylated by phosphatidic acid phosphatase (PAP) and diacylglycerol (DAG) is generated. Diacylglycerol acyltransferase (DGAT) catalyzes the final step in TAG synthesis using diacylglycerol and acyl-CoA as substrates (W. L. Yu et al., 2011). It has been proposed that TAG biosynthesis takes place either in the chloroplast or in the endoplasmic reticulum (ER) (figure 2).

The first step of TAG synthesis is catalyzed by GPAT and is considered the ratelimiting reaction (J. Yu et al., 2018). Balamurugan et al. (2017) and Niu et al. (2016) reported that overexpression of endogenous GPAT in Phaeodacty/um tricornutum increased neutral lipid content by 1.8 and 2 -folds compared to wild type strains, respectively. Both studies showed significantly higher levels of unsaturated fatty acids and no effect on growth rates. Recently, a glycerol-3phosphate acyltransferase 2 (GPAT2) isoform was identified and overexpressed in Phaeodactylum tricornutum, showing similar results (X. Wang et al., 2020). Wang et al. (2020) observed a reduced carbohydrate and protein content, and 2.9-fold increased TFA and TAG content in transgenic strains. GPAT2 overexpressing strains showed no growth impairment, and an altered lipid composition with an increase of $\mathrm{C} 16: 0$ and a decrease of both $\mathrm{C} 18: 0$ and unsaturated fatty acid content.

Furthermore, Fukuda et al. (2018) demonstrated that endogenous overexpression of GPAT in the red alga Cyanidioschyzon merolae can influence lipid composition. Although the TFA content was not significantly changed in comparison to WT strains, relative abundance of $\mathrm{C} 18: 2$ in the sn-1/sn-3 positions of TAGs were significantly increased without a negative impact on 
growth, and thus leading to 56.1-fold increase in maximum TAG productivities in mutant strains. Additionally, heterologous expression of GPAT in microalgae has shown a successful increase in lipid accumulation. Overexpression of GPAT from Saccharomyces cerevisiae and Lobosphaera incise in Chlamydomonas reinhardtii resulted in an increase of $67.5 \%$ and $50 \%$ for TFA and TAG, respectively. Additionally, an increase in long-chain saturated fatty acids, and decrease in PUFAs without any inhibition on growth were observed in these mutants (Iskandarov et al., 2016; Chaogang Wang et al., 2018).

Overexpression of LPAT, the next enzyme in the Kennedy pathway, has also been reported to improve the lipid content in $C$. reinhardtii . Yamaoka et al. (2016) investigated the effect of the endogenous overexpression of a plastidial LPAT and showed an increase of $20 \%$ in TAGs under nitrogen-deficient conditions. Likewise, Wang et al. (2018) reported that heterologous expression of LPAT from Brassica napus in C. reinhardtii increased lipid content by $44.5 \%$ without affecting growth.

Furthermore, Deng et al. (2013) have studied the biological activity of PAP in C. reinhardtii and its impact on lipid biosynthesis. Phosphatidic acid phosphatase dephosphorylates phosphatidic acid into DAG, which is the main precursor for TAG synthesis. Overexpression and knockdown of PAP resulted in 7.5-21.8\% increase and $2.4-17.4 \%$ decrease in lipid content, respectively, demonstrating its importance in the lipid biosynthesis pathway (X. D. Deng et al., 2013).

The last step of the TAG biosynthesis pathway is catalyzed by diacylglycerol acyltransferase (DGAT). Overexpression of genes encoding DGAT has so far been the most frequently employed strategy for increasing TAG contents in microalgae. Although the first attempt performed in the model microalga $C$. reinhardtii (La Russa et al., 2012) did not lead to significant change in lipid content, later studies demonstrated an increase of TFA between 1.5- to 2.5fold in the same species (Ahmad et al., 2015; X. D. Deng et al., 2012; Iwai et al., 2014). Moreover, overexpression of DGAT in Nannochloropsis strains has been reported to increase the TAG content between 1.3 - to 2.4 -fold or to 
enhance the TFA content (Tracey A. Beacham \& Ali, 2016; Iwai et al., 2015; D. W. Li et al., 2016; H. Wei et al., 2017; Zienkiewicz et al., 2017). The same strategy has been used for the diatom Phaeodactylum, resulting in 1.3- to 2.3fold increases in TAG content (Dinamarca et al., 2017; Y.-F. Niu et al., 2013; Zulu et al., 2017). While most of the research focused on species from the genus of Chlamydomonas, Nannochloropsis and Phaeodactylum, similarly promising results were achieved for microalgae belonging to Thalassiosira, Scenedesmus, Neochloris, Tetraselmis and Coccomyxa genera (C.-Y. Chen et al., 2016; Kasai et al., 2018; Klaitong et al., 2017; Manandhar-Shrestha \& Hildebrand, 2015; Muñoz et al., 2019; Úbeda-Mínguez et al., 2017).

Several studies have further shown promising results when using a multi-gene expression approach. Wang et al. (2018) have demonstrated that dual expression of LPAT and GPAT in P. tricornutum leads to 2.4- and 2.3-fold increase in TFA and TAG content, respectively. Likewise, dual expression of DGAT and GPAT was reported to increase TFA by 2.6 -fold in the same strain (Zou et al., 2018). Furthermore, simultaneous expression of DGAT, GPAT and LPAT was reported to increase TFA and TAG content by 1.2 -fold in $N$. oleoabundans (Muñoz et al., 2019). Similarly, the overexpression of endogenous LPAT1 and DGAT2 in the same species lead to 1.6- and 2.1-fold increase of TFAs and TAG, respectively (Chungjatupornchai \& Fa-aroonsawat, 2020). Studies have shown that overexpression of the entire pathway including $D G A T, G P A T, L P A T$ and PAP genes can also have a significant impact on lipid biosynthesis by increasing TAG content between 2 and 2.3-fold in Chlorella species (Chien et al., 2015; Hsieh et al., 2012).

Table 2. Genetic engineering of Kennedy pathway for enhanced lipid production

\begin{tabular}{|c|c|c|c|c|c|}
\hline Algal Strain & $\begin{array}{l}\text { Targeted } \\
\text { Genes }\end{array}$ & Strategy & $\begin{array}{l}\text { Effect on lipid } \\
\text { synthesis }\end{array}$ & Comments & References \\
\hline C. reinhardtii & $\begin{array}{l}\text { CrDGAT2a } \\
\text { CrDGAT2b } \\
\text { CrDGAT2c }\end{array}$ & $\mathrm{OE}$ & $\begin{array}{l}\text { No change in lipid } \\
\text { content }\end{array}$ & $\begin{array}{l}\text { Enhanced mRNA } \\
\text { expression of DGAT } \\
\text { genes did not boost } \\
\text { TAG accumulation } \\
\text { and did not result in } \\
\text { alterations of fatty } \\
\text { acid profiles }\end{array}$ & $\begin{array}{l}\text { (La Russa et } \\
\text { al., 2012) }\end{array}$ \\
\hline C. reinhardtii & $\begin{array}{l}\text { CrDGAT2.1 } \\
\text { CrDGAT2.5 }\end{array}$ & $\mathrm{OE}$ & $\begin{array}{l}\text { DGAT2-1 and DGAT2- } \\
5 \text { increased TFA by } \\
27.25 \% \text { and } 48 \%\end{array}$ & $\begin{array}{l}\text { Silencing } \\
\text { CrDGAT2- } \\
1 \text { or CrDGAT2-5 }\end{array}$ & $\begin{array}{l}\text { (X. D. Deng } \\
\text { et al., 2012) }\end{array}$ \\
\hline
\end{tabular}




\begin{tabular}{|c|c|c|c|c|c|}
\hline & & & $\begin{array}{l}\text { respectively, during } \\
\text { replete conditions. }\end{array}$ & $\begin{array}{l}\text { decreased lipid } \\
\text { content by } 16 \%- \\
24 \% \text { or } 28 \%-37 \% \text {, } \\
\text { respectively }\end{array}$ & \\
\hline P. tricornutum & $D G A T 2$ & OE & $\begin{array}{l}35 \% \text { increased } \\
\text { neutral lipids. EPA } \\
\text { increased by } 76.2 \% \text {. }\end{array}$ & $\begin{array}{l}\text { Growth rate of } \\
\text { transgenic strains } \\
\text { remained similar to } \\
\text { WT }\end{array}$ & $\begin{array}{l}\text { (Y.-F. Niu et } \\
\text { al., 2013) }\end{array}$ \\
\hline C. reinhardtii & CrDGTT4 & OE & $\begin{array}{l}\text { 2.5-fold increased } \\
\text { TAG during P } \\
\text { starvation }\end{array}$ & $\begin{array}{l}\text { Enhanced } \quad \text { TAG } \\
\text { accumulation with } \\
\text { a slight increase in } \\
\text { C18:1 content, } \\
\text { using a P } \\
\text { starvation- } \\
\text { inducible promoter }\end{array}$ & $\begin{array}{l}\text { (Iwai et al., } \\
\text { 2014) }\end{array}$ \\
\hline C. reinhardtii & BnDGAT2 & $\mathrm{HE}$ & $\begin{array}{l}7 \% \text { decrease in total } \\
\text { saturated fatty acids. } \\
7 \% \text { increase in overall } \\
\text { PUFA content }\end{array}$ & $\begin{array}{l}\text { Growth rate of } \\
\text { transformants } \\
\text { similar to wild type } \\
\text { strains }\end{array}$ & $\begin{array}{l}\text { (Ahmad et } \\
\text { al., 2015) }\end{array}$ \\
\hline T. pseudonana & DGAT2 & $\mathrm{OE}$ & $\begin{array}{l}\text { 1.52-1.95-fold } \\
\text { increased TAG } \\
\text { content. Increased } \\
\text { C16:1, C16:2, C20:5 } \\
\text { and C22:6 fatty acid } \\
\text { contents }\end{array}$ & $\begin{array}{l}\text { No change in } \\
\text { growth } \\
\text { compared to wild } \\
\text { type strains }\end{array}$ & $\begin{array}{l}\text { (Manandhar } \\
\text {-Shrestha \& } \\
\text { Hildebrand, } \\
\text { 2015) }\end{array}$ \\
\hline $\begin{array}{l}\text { Nannochloropsis } \\
\text { strain NIES-2145 }\end{array}$ & CrDGTT4 & $\mathrm{HE}$ & $\begin{array}{l}\text { 1.3-1.7-fold } \\
\text { increased TAG } \\
\text { content. The levels of } \\
\text { C16:0, C16:1, C18:1 } \\
\omega-9, \text { and C20:3 } \omega-6 \\
\text { fatty acids increased, } \\
\text { whereas C16:3, } \\
\text { C18:2 } \omega-6, \text { C20:4 } \omega- \\
6 \text { and C20:5 fatty } \\
\text { acids decreased } \\
\text { under P starvation } \\
\text { conditions }\end{array}$ & $\begin{array}{l}\text { No significant } \\
\text { changes in TFA and } \\
\text { total polar lipids } \\
\text { were observed }\end{array}$ & $\begin{array}{l}\text { (Iwai et al., } \\
\text { 2015) }\end{array}$ \\
\hline N. oceanica & DGAT2 & OE & $\begin{array}{l}69 \% \text { and } 129 \% \\
\text { increased } \\
\text { content TAG } \\
\text { replete and deplete } \\
\text { conditions. } 74 \% \text { and } \\
53 \% \text { decreased PUFA } \\
\text { and MUFA content, } \\
\text { respectively }\end{array}$ & $\begin{array}{l}\text { DGAT2 } \\
\text { overexpression did } \\
\text { not show negative } \\
\text { impact on algal } \\
\text { growth }\end{array}$ & $\begin{array}{l}\text { (D. W. Li et } \\
\text { al., 2016) }\end{array}$ \\
\hline N. salina & $D G A 1$ & $\mathrm{HE}$ & $\begin{array}{l}18-38 \% \text { increased } \\
\text { TFA during replete } \\
\text { conditions. } 75 \% \\
\text { increased } \\
\text { productivities in mid- } \\
\text { exponential phase }\end{array}$ & $\begin{array}{l}\text { Reduced growth } \\
\text { rate in transgenic } \\
\text { strains compared to } \\
\text { wild type. }\end{array}$ & $\begin{array}{l}\text { (Tracey A. } \\
\text { Beacham \& } \\
\text { Ali, 2016) }\end{array}$ \\
\hline S. obliquus & DGTT1 & $\mathrm{HE}$ & 2-fold increased TFA & $\begin{array}{l}29 \% \text { higher } \\
\text { biomass } \\
\text { concentration than } \\
\text { that of the wild type }\end{array}$ & $\begin{array}{l}\text { (C.-Y. Chen } \\
\text { et al., 2016) }\end{array}$ \\
\hline P. tricornutum & $D G A T 2 D$ & $\mathrm{OE}$ & $\begin{array}{l}\text { 1.6-2-fold higher total } \\
\text { lipid content }\end{array}$ & $\begin{array}{l}50-100 \text {-fold higher } \\
\text { DGAT2D mRNA } \\
\text { levels and } 30-50- \\
\text { fold increased } \\
\text { enzyme } \\
\text { abundance. } 15 \% \\
\text { decrease in growth } \\
\text { rate }\end{array}$ & $\begin{array}{l}\text { (Dinamarca } \\
\text { et al., 2017) }\end{array}$ \\
\hline N. oleoabundans & NeoDGAT2 & OE & $\begin{array}{l}\text { 1.6-2.3-fold } \\
\text { increased TFA and } \\
1.6-3.2 \text {-fold }\end{array}$ & $\begin{array}{l}\text { No significant effect } \\
\text { on growth }\end{array}$ & $\begin{array}{l}\text { (Klaitong et } \\
\text { al., 2017) }\end{array}$ \\
\hline
\end{tabular}




\begin{tabular}{|c|c|c|c|c|c|}
\hline & & & $\begin{array}{l}\text { increased total lipid } \\
\text { productivity. 1.8-3.2- } \\
\text { fold increased TAG } \\
\text { and 1.6-4.3-fold } \\
\text { increased total TAG } \\
\text { productivity }\end{array}$ & & \\
\hline N. oceanica & $D G A T 1 A$ & $\mathrm{OE}$ & $\begin{array}{l}39 \% \text { increase in TAG } \\
\text { content under } \\
\text { nitrogen-depletion. } \\
2.4-\text { fold increased } \\
\text { TAG under nitrogen- } \\
\text { replete. TAG yield } \\
47 \% \text { greater than for } \\
\text { the wild type strain }\end{array}$ & $\begin{array}{l}\text { NoDGAT1A } \\
\text { overexpression did } \\
\text { not show negative } \\
\text { impact on growth. } \\
\text { NoDGAT1A } \\
\text { knockdown caused } \\
25 \% \text { decline in TAG } \\
\text { content during } \\
\text { nitrogen depletion. }\end{array}$ & $\begin{array}{l}\text { (H. Wei et } \\
\text { al., 2017) }\end{array}$ \\
\hline N. oceanica & NoDGTT5 & $\mathrm{OE}$ & $\begin{array}{l}1.75 \text {-fold increased } \\
\text { TAG under } \mathrm{N} \text {-replete } \\
\text { conditions }\end{array}$ & $\begin{array}{l}50 \% \text { decreased } \\
\text { growth rate in } \\
\text { transgenic strains } \\
\text { compared to wild } \\
\text { type }\end{array}$ & $\begin{array}{l}\text { (Zienkiewicz } \\
\text { et al., 2017) }\end{array}$ \\
\hline T. chui & $\begin{array}{l}\text { EPDGAT1 } \\
\text { SCDGAT2 }\end{array}$ & $\mathrm{HE}$ & $\begin{array}{l}40-115 \% \text { increase in } \\
\text { TAG content }\end{array}$ & $\begin{array}{l}\text { No significant effect } \\
\text { on growth }\end{array}$ & $\begin{array}{l}\text { (Úbeda- } \\
\text { Mínguez et } \\
\text { al., 2017) }\end{array}$ \\
\hline P. tricornutum & $\begin{array}{l}\text { ScDGA1 } \\
\text { AtOLEO3 }\end{array}$ & $\mathrm{HE}$ & $\begin{array}{l}\text { SCDGA1 and AtOLEO3 } \\
\text { increased TAG } \\
\text { content 2.3- and 1.4- } \\
\text { fold, respectively. Co- } \\
\text { expression resulted in } \\
3.6 \text {-fold increased } \\
\text { TAG content. TAG } \\
\text { productivity increased } \\
\text { by } 2 \text {-folds under N- } \\
\text { stress }\end{array}$ & $\begin{array}{l}\text { Fatty acid } \\
\text { composition } \\
\text { remained } \\
\text { unchanged in TFA } \\
\text { and TAG }\end{array}$ & $\begin{array}{l}\text { (Zulu et al., } \\
\text { 2017) }\end{array}$ \\
\hline Coccomyxa sp. & $\begin{array}{l}\text { CDGAT2d } \\
\text { CFAT1 }\end{array}$ & $\mathrm{OE}$ & $\begin{array}{l}\text { FAT1 and DGAT2 } \\
\text { increased TFA content } \\
1.1-\text { and 1.1-fold, } \\
\text { respectively. Co- } \\
\text { expression resulted in } \\
1.12 \text {-fold increased } \\
\text { TFA content and 1.4- } \\
\text { fold increased TFA } \\
\text { productivity }\end{array}$ & $\begin{array}{l}\text { Single expression } \\
\text { did not impair } \\
\text { growth rates. Co- } \\
\text { expression } \\
\text { increased growth } \\
\text { rate by } 1.27 \text {-folds }\end{array}$ & $\begin{array}{l}\text { (Kasai et al., } \\
\text { 2018) }\end{array}$ \\
\hline C. reinhardtii & CrLPAAT1 & $\mathrm{OE}$ & $\begin{array}{l}20 \% \text { increase in TAG } \\
\text { content under } \\
\text { nitrogen-deficient } \\
\text { conditions }\end{array}$ & 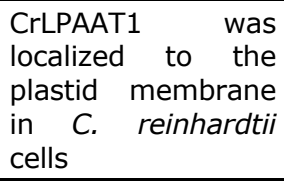 & $\begin{array}{l}\text { (Yamaoka et } \\
\text { al., 2016) }\end{array}$ \\
\hline C. reinhardtii & CrPAP2 & $\mathrm{OE}$ & $\begin{array}{l}7.5-21.8 \% \text { increased } \\
\text { TFA content }\end{array}$ & $\begin{array}{l}\text { Silencing of the } \\
\text { CrPAP2 } \text { gene } \\
\text { resulted in } 2.4- \\
17.4 \% \text { decrease in } \\
\text { total lipid content }\end{array}$ & $\begin{array}{l}\text { (X. D. Deng } \\
\text { et al., 2013) }\end{array}$ \\
\hline P. tricornutum & GPAT & $\mathrm{OE}$ & $\begin{array}{l}1.34-2 \text {-fold increase } \\
\text { in TAG content under } \\
\mathrm{N}-\text { deplete conditions. } \\
35 \%, 12 \% \text { and } 45 \% \\
\text { decrease in SFA and } \\
\text { MUFAs, respectively. } \\
41 \% \text { increased PUFA } \\
\text { content }\end{array}$ & $\begin{array}{l}\text { Overexpression did } \\
\text { not show significant } \\
\text { effect on growth } \\
\text { and slight increase } \\
\text { in photosynthetic } \\
\text { efficiency }\end{array}$ & $\begin{array}{l}\text { (Y. F. Niu et } \\
\text { al., 2016) }\end{array}$ \\
\hline C. reinhardtii & LIGPAT & $\mathrm{HE}$ & $\begin{array}{l}50 \% \text { increase in TAG } \\
\text { content. Increase in } \\
\text { C18:1 n-9 and } 1 C 6: 0 \text {, } \\
\text { decrease in C18:3 n- } \\
3 \text { and } C 16: 4 n-3\end{array}$ & $\begin{array}{l}\text { Overexpression did } \\
\text { not have significant } \\
\text { effect on growth }\end{array}$ & $\begin{array}{l}\text { (Iskandarov } \\
\text { et al., 2016) }\end{array}$ \\
\hline
\end{tabular}




\begin{tabular}{|c|c|c|c|c|c|}
\hline P. tricornutum & AGPAT1 & $\mathrm{OE}$ & $\begin{array}{l}\text { 1.81-fold increase in } \\
\text { TAG content and } \\
2.04 \text {-fold higher lipid } \\
\text { yield }\end{array}$ & $\begin{array}{l}\text { No effect on } \\
\text { growth. Decreased } \\
\text { protein } \begin{array}{l}\text { and } \\
\text { carbohydrate } \\
\text { content }\end{array} \\
\end{array}$ & $\begin{array}{l}\text { (Balamurug } \\
\text { an et al., } \\
2017 \text { ) }\end{array}$ \\
\hline C. merolae & CMGPAT1 & $\mathrm{OE}$ & $\begin{array}{l}\text { No significant effect } \\
\text { on TFA. 19-fold } \\
\text { increased } \\
\text { content and 56.1-fold } \\
\text { increase in TAG } \\
\text { productivities }\end{array}$ & $\begin{array}{l}\text { Overexpression did } \\
\text { not show significant } \\
\text { effect on growth }\end{array}$ & $\begin{array}{l}\text { (Fukuda et } \\
\text { al., 2018) }\end{array}$ \\
\hline P. tricornutum & GPAT2 & $\mathrm{OE}$ & $\begin{array}{l}\text { 2.9-fold increase in } \\
\text { TAG content. Altered } \\
\text { fatty acid profile in } \\
\text { TAGs with increase of } \\
\text { C16:0 }\end{array}$ & $\begin{array}{l}\text { No effect on growth } \\
\text { and photosynthetic } \\
\text { efficiency. } \\
\text { Enhanced tolerance } \\
\text { to hyposaline and } \\
\text { chilling conditions }\end{array}$ & $\begin{array}{l}\text { (X. Wang et } \\
\text { al., 2020) }\end{array}$ \\
\hline C. reinhardtii & $\begin{array}{l}\text { LPAAT } \\
\text { GPD1 }\end{array}$ & $\mathrm{HE}$ & $\begin{array}{l}\text { LPAAT and GPD1 } \\
\text { increased TFA content } \\
\text { by } 44.5 \% \text { and } 67.5 \% \text {. } \\
\text { Increase in long-chain } \\
\text { saturated fatty acids } \\
\text { and decreased } \\
\text { unsaturated fatty } \\
\text { acids }\end{array}$ & $\begin{array}{l}\text { No effect on } \\
\text { growth. Decreased } \\
\text { protein content }\end{array}$ & $\begin{array}{l}\text { (Chaogang } \\
\text { Wang et al., } \\
\text { 2018) }\end{array}$ \\
\hline P. tricornutum & $\begin{array}{l}\text { GPAT1 } \\
\text { LPAAT1 }\end{array}$ & $\mathrm{OE}$ & $\begin{array}{l}\text { Dual expression } \\
\text { resulted in } 2.3 \text {-fold } \\
\text { increased } \\
\text { content under N- } \\
\text { replete conditions. } \\
2.7 \text { and } 3 \text {-fold } \\
\text { increase in TFA and } \\
\text { TAG productivities, } \\
\text { respectively }\end{array}$ & $\begin{array}{l}\text { Dual expression } \\
\text { resulted in } 1.5 \text {-fold } \\
\text { increase in growth } \\
\text { rate during mid-log } \\
\text { phase }\end{array}$ & $\begin{array}{l}\text { (X. Wang et } \\
\text { al., 2018) }\end{array}$ \\
\hline P. tricornutum & $\begin{array}{l}\text { DGAT2 } \\
\text { GPAT }\end{array}$ & $\mathrm{OE}$ & $\begin{array}{l}2.6 \text {-fold increased } \\
\text { TFA compared to WT, } \\
\text { reaching up to } 57.5 \% \\
\text { DCW. Increased } \\
\text { MUFA and PUFA } \\
\text { content }\end{array}$ & $\begin{array}{lr}\text { No effect } & \text { on } \\
\text { growth. } & \text { Slight } \\
\text { increase } & \text { in } \\
\text { photosynthetic } & \\
\text { efficiency } & \end{array}$ & $\begin{array}{l}\text { (Zou et al., } \\
2018 \text { ) }\end{array}$ \\
\hline N. oleoabundans & $\begin{array}{l}\text { LPAAT1 } \\
\text { DGAT2 }\end{array}$ & $\mathrm{OE}$ & $\begin{array}{l}\text { Co-expression } \\
\text { resulted in } 1.6 \text { and } \\
2.1 \text {-fold increased } \\
\text { TFA and TAG content, } \\
\text { respectively. } 1.9 \text { and } \\
2.1 \text {-fold increased } \\
\text { TFA and TAG } \\
\text { productivities, } \\
\text { respectively }\end{array}$ & $\begin{array}{l}\text { Co-expression did } \\
\text { not affect growth }\end{array}$ & $\begin{array}{l}\text { (Chungjatup } \\
\text { ornchai \& } \\
\text { Fa- } \\
\text { aroonsawat, } \\
\text { 2020) }\end{array}$ \\
\hline N. oleoabundans & $\begin{array}{l}\text { GPAT } \\
\text { LPAAT } \\
D G A T\end{array}$ & $\mathrm{HE}$ & $\begin{array}{l}\text { Single-gene } \\
\text { expression resulted in } \\
1.3 \text { and 1.4-fold } \\
\text { increased TFA and } \\
\text { TAG content, } \\
\text { respectively. Multi- } \\
\text { gene expression } \\
\text { resulted in 1.2-fold } \\
\text { increase in TFA and } \\
\text { TAG }\end{array}$ & $\begin{array}{l}\text { Single expression } \\
\text { did not affect } \\
\text { growth and } \\
\text { photosynthetic } \\
\text { efficiency. Multi- } \\
\text { gene expression } \\
\text { decreased growth } \\
\text { rate, } \\
\text { photosynthetic } \\
\text { efficiency, } \\
\text { carbohydrates and } \\
\text { protein content }\end{array}$ & $\begin{array}{l}\text { (Muñoz et } \\
\text { al., 2019) }\end{array}$ \\
\hline C. minutissima & $\begin{array}{l}\text { G3PDH } \\
\text { GPAT } \\
\text { LPAAT } \\
\text { PAP } \\
\text { DGAT }\end{array}$ & $\mathrm{HE}$ & $\begin{array}{l}\text { Quintuple-gene } \\
\text { expression resulted in } \\
\text { 2-fold increased TAG } \\
\text { content and } \\
\text { increased }\end{array}$ & $\begin{array}{l}\text { Single-gene } \\
\text { constructs showed } \\
\text { little effect on } \\
\text { enhancing TAG } \\
\text { production }\end{array}$ & $\begin{array}{l}\text { (Hsieh et al., } \\
\text { 2012) }\end{array}$ \\
\hline
\end{tabular}




\begin{tabular}{|c|c|c|c|c|c|}
\hline & & & $\begin{array}{ll}\text { productivity. } & \text { 2-fold } \\
\text { increased TFA } & \end{array}$ & & \\
\hline Chlorella sp. & $\begin{array}{l}\text { GPAT } \\
\angle P A A T \\
P A P \\
D G A T\end{array}$ & $\mathrm{HE}$ & $\begin{array}{l}\text { Quadruple-gene } \\
\text { expression resulted in } \\
2.3 \text {-fold increased } \\
\text { TAG content }\end{array}$ & $\begin{array}{l}\text { Similar growth and } \\
\text { TAG productivity }\end{array}$ & $\begin{array}{l}\text { (Chien et al., } \\
\text { 2015) }\end{array}$ \\
\hline
\end{tabular}

\subsection{Polyunsaturated fatty acids (PUFAs) metabolism}

Long-chain polyunsaturated fatty acids (LC-PUFAs) are aliphatic carbon chains consisting of 18,20 or 22 carbons. They are classified into two main groups based on the position of the first double bond at the $3^{\text {rd }}(\omega-3)$ or $6^{\text {th }}(\omega-6)$ carbon atom counting from the methyl end. In nature, LC-PUFAs are found mainly in fish oils and they are essential in human diet. In addition to the benefits of physical and mental wellbeing, LC-PUFAs are also reported to promote the development of infant health and prevention of diseases (Zárate et al., 2017). Although they can be obtained from marine fish oils, increased pollution of the oceans, climate variations and overfishing are causing a decline in wild fish stocks and thereby reduce the supply required to meet the global demand (Nations, 2016).

Microalgae are the primary producers of LC-PUFAs in aquatic environments. Such lipids serve as building blocks and are mainly present in photosynthetic membranes (thylakoids), but they can also be found in lipid bodies as a response to unexpected environmental changes or during nutrient starvation (Solovchenko, 2012). Commercially relevant LC-PUFAs such as eicosapentaenoic (EPA), docosahexaenoic (DHA) and arachidonic acid (ARA) can be found predominantly in marine or salt-tolerant microalgae species such as Phaeodactylum tricornutum and Nannochloropsis oceanica (Boelen et al., 2013).

Genetic engineering of various microalgae species has enhanced the accumulation of industrially relevant PUFAs, by regulating the expression of genes encoding fatty acid desaturases (FADs) and elongases (ELOs). These enzymes catalyze a series of desaturation and elongation steps of aliphatic carbon chains that lead to PUFA formation (figure 2) (Khozin-Goldberg et al., 
2011). For instance, Zäuner et al. (2012) have demonstrated that overexpression of $\triangle 4-F A D$ in $C$. reinhardtii ( $\mathrm{Cr} \triangle 4 \mathrm{FAD}$ ) increased the total MGDG content from $9 \mathrm{fmol} / \mathrm{cell}$ to $15 \mathrm{fmol} / \mathrm{cell}$ compared to the WT. This modification did not alter TFA content and resulted in $12 \%$ increase of $\mathrm{C} 16: 4$ and $8 \%$ increase of $18: 3$. On the other hand, the $\mathrm{Cr} \triangle 4 \mathrm{FAD}$ knockdown decreased the fraction of C16:4 and C18:3 in TFAs (Zäuner et al., 2012).

An endogenous $\triangle 5$-FAD was overexpressed in P. tricornutum CCMP2561 (Peng et al., 2014), resulting in an increase of neutral lipid content up to $65 \%$ with no effect on growth. An increase of $75 \%$ of MUFAs and 64\% PUFAs was observed with a $16 \%$ decline in saturated fatty acids (SFAs), indicating redirection of lipid flux towards PUFA synthesis. EPA was increased by $58 \%$ and $0.44 \mathrm{mg} / \mathrm{g} \mathrm{dcw}$ of DHA was produced (Peng et al., 2014). Similarly, Hamilton et al. (2014) found that DHA content of $P$. tricornutum was substantially increased by heterologous expression of $\triangle 5$-ELO from Ostreococcus tauri. In this case, DHA was increased by 8 -fold and accounted for $10.4 \%$ of the TFAs during the stationary phase, while EPA levels almost halved compared to the WT. Moreover, co-expression of $\triangle 5$-ELO and $\triangle 6-F A D$ from $O$. tauri further increased DHA levels to $11.4 \%$ of TFA (M. L. Hamilton et al., 2014). Furthermore, Hamilton et al. (2016) co-expressed the $\Delta 5$-ELO and a glucose transporter from Physcomitrella patens in P. tricornutum, enabling glucose uptake in a phototrophic microalga that naturally does not grow on glucose (M. Hamilton et al., 2016). Under heterotrophic conditions, transformants produced $7.3 \%$ of DHA per TFAs and up to $9.1 \%$ of docosapentaenoic acid (DPA), with no significant variations in the EPA content. Moreover, after transferring the cultures from heterotrophic to phototrophic conditions, both EPA and DHA levels further increased, reaching $19 \%$ and $9.2 \%$ of TFAs, respectively. Under mixotrophic cultivation, EPA and DHA levels increased 1.7- and 1.1-fold, respectively, compared to strains expressing only a $\triangle 5$-ELO (M. Hamilton et al., 2016).

In an attempt to increase DHA levels, Haslam et al. (2020) characterized and overexpressed native $P$. tricornutum DGAT2B, which showed preferences for C16 and LC-PUFA acyl groups (Haslam et al., 2020). In the same study, the 
combined expression of DGAT2B and $\triangle 5$-ELO led to an increased incorporation of PUFAs into TAGs. The overexpression of endogenous $\triangle 6-F A D$ in $P$. tricornutum increased EPA contents by $48 \%$ (B. H. Zhu et al., 2017). Although transformants did not show any change in DHA content, SFA content was increased by $28 \%$ and MUFA content was slightly reduced. Similarly, overexpression of $\triangle 6$-FAD from Thalassiosira pseudonana (TpFAD6) in D. salina enhanced EPA up to $21.3 \mathrm{mg} / \mathrm{L}$ compared to $1.6 \mathrm{mg} / \mathrm{L}$ in the WT (Shi et al., 2018). Moreover, by improving the carbon availability during cultivation, EPA content was further enhanced by 25 -fold to $554 \mathrm{mg} / \mathrm{L}$.

An endogenous $\triangle 12-F A D$ was overexpressed in $N$. oceanica, which caused a decrease in OA (substrate for $\triangle 12-F A D$ ) and increase in LA in TAGs and other lipid classes of transgenic strains (Kaye et al., 2015). Furthermore, ARA levels were increased in different lipid classes, indicating an increase in carbon flux towards the omega-6 PUFA biosynthesis pathway. Poliner et al. (2018) observed a $25 \%$ increase in EPA in $N$. oceanica strains overexpressing a $\triangle 12-$ FAD or $\Delta 5$-FAD. Co-expression of $\Delta 9$ and $\Delta 12$-FADs or $\Delta 9, \Delta 5$ and $\Delta 12$-FADs did not improve the EPA content compared to the previously attained $25 \%$. Although cell growth was increased in all transformant lines, the total fatty acid content per cell was decreased compared to the wild type strain (Poliner et al., 2018).

Norashikin et al. (2018) reported an effect of overexpressing the endogenous $\omega-3-F A D$ in C. vulgaris. A significant increase in TFA from $40 \%$ to $47 \%$ was observed in transformants when grown under nitrogen-deficient conditions. Transformants also showed a slight increase in a-linolenic acid (ALA) content from $8 \%$ to $10.8 \%$ of TFA compared to the WT (Norashikin et al., 2018).

Other strategies such as increasing fatty acid precursors, enhancing the TAG biosynthesis pathway, optimizing the supply of cofactors and downregulating lipid catabolism have also shown promising results in enhancing LC-PUFAs and they are reported in other sections of this review. 
Table 3. Genetic engineering of PUFA metabolism for enhanced lipid production.

\begin{tabular}{|c|c|c|c|c|c|}
\hline Algal Strain & $\begin{array}{l}\text { Targeted } \\
\text { Genes }\end{array}$ & Strategy & $\begin{array}{l}\text { Effect on lipid } \\
\text { synthesis }\end{array}$ & Comments & References \\
\hline C. reinhardtii & $C r \triangle 4 F A D$ & $\mathrm{OE}$ & $\begin{array}{l}\text { No significant effect } \\
\text { on TFA. Increase in } \\
\text { C16:4 and total } \\
\text { MGDG }\end{array}$ & $\begin{array}{l}\text { Cr } \triangle 4 F A D \text { knockdown } \\
\text { decreased } C 16: 4 \text { and } \\
\text { C18:3 in TFA }\end{array}$ & $\begin{array}{l}\text { (Zäuner et } \\
\text { al., 2012) }\end{array}$ \\
\hline P. tricornutum & PtD5b & $\mathrm{OE}$ & $\begin{array}{l}75 \% \text { and } 64 \% \\
\text { increased MUFA and } \\
\text { PUFA content. 58\% } \\
\text { and } 65 \% \text { increased } \\
\text { EPA and neutral } \\
\text { lipids, respectively }\end{array}$ & $\begin{array}{l}\text { No significant effect } \\
\text { on growth }\end{array}$ & $\begin{array}{l}\text { (Peng et al., } \\
\text { 2014) }\end{array}$ \\
\hline P. tricornutum & $\begin{array}{l}\text { OtElo5 } \\
\text { OtD6N }\end{array}$ & $\mathrm{HE}$ & $\begin{array}{l}\text { OtElo5 expression } \\
\text { increased } \\
\text { content 8-fold. Co- } \\
\text { expression of OtElo5 } \\
\text { and OtD6N increased } \\
\text { DHA up to } 11.4 \% \text { of } \\
\text { TFA }\end{array}$ & $\begin{array}{l}\text { Accumulation of DHA } \\
\text { in TAGs }\end{array}$ & $\begin{array}{ll}\text { (M. } & \text { L. } \\
\text { Hamilton } & \text { et } \\
\text { al., 2014) } & \end{array}$ \\
\hline P. tricornutum & $\begin{array}{l}\text { OtElo5 } \\
\text { Ppglut1 }\end{array}$ & $\mathrm{HE}$ & $\begin{array}{l}32.2 \% \text { PUFA per TFA } \\
\text { produced under } \\
\text { mixotrophic } \\
\text { conditions. } \\
\text { phototrophic Under } \\
\text { conditions, up to } \\
\begin{array}{l}36.5 \% \text { and } 23.6 \% \\
\text { DHA and EPA in TFA, } \\
\text { respectively }\end{array}\end{array}$ & $\begin{array}{l}\text { Ppglut1 allowed } \\
\text { growth in the dark if } \\
\text { medium } \\
\text { supplemented with } \\
\text { glucose }\end{array}$ & $\begin{array}{l}\text { (M. Hamilton } \\
\text { et al., 2016) }\end{array}$ \\
\hline N. oceanica & NoD12 & OE & $\begin{array}{l}50-75 \% \text { increased AA } \\
\text { under N-starvation } \\
\text { conditions. } 32.6 \% \\
\text { increased LA in PC }\end{array}$ & $\begin{array}{l}\text { Overexpression under } \\
\text { the control of stress- } \\
\text { inducible endogenous } \\
\text { lipid droplet surface } \\
\text { protein } \quad \text { (LDSP) } \\
\text { promoter, (Higher } \\
\text { expression under N- } \\
\text { starvation conditions) }\end{array}$ & $\begin{array}{l}\text { (Kaye et al., } \\
2015 \text { ) }\end{array}$ \\
\hline P. tricornutum & $D 6 F A D$ & OE & $\begin{array}{l}47.66 \% \text { increased } \\
\text { EPA content, reaching } \\
\text { up to } 38.101 \mathrm{mg} / \mathrm{g} \\
\text { dcw. 16.4-18.64\% } \\
\text { increased TFA } \\
\text { compared to wild type } \\
\text { strain }\end{array}$ & $\begin{array}{l}\text { Slight reduction in } \\
\text { specific growth rate }\end{array}$ & $\begin{array}{l}\text { (B. H. Zhu et } \\
\text { al., 2017) }\end{array}$ \\
\hline D. salina & $\begin{array}{l}\text { TpFADS6 } \\
D s F A D S 6\end{array}$ & $\mathrm{OE}, \mathrm{HE}$ & $\begin{array}{l}\text { Enhanced EPA up to } \\
21.3 \mathrm{mg} / \mathrm{L} \text {, compared } \\
\text { to } 1.6 \mathrm{mg} / \mathrm{L} \text { in the } \\
\text { WT. Up to } 91,193 \\
\text { and } 554 \mathrm{mg} / \mathrm{L} \text { EPA in } \\
\text { TFA when } \\
\text { supplemented with } \\
\text { myoinositol, } \mathrm{CO}_{2} \text { and } \\
\text { PeSM, respectively }\end{array}$ & $\begin{array}{l}\text { Use of myoinositol, } \\
\mathrm{CO}_{2} \text { and PeSM to } \\
\text { promote growth }\end{array}$ & $\begin{array}{l}\text { (Shi et al., } \\
\text { 2018) }\end{array}$ \\
\hline C. vulgaris & $\omega-3$ FAD & $\mathrm{OE}$ & $\begin{array}{l}7 \% \text { increased TFA } \\
\text { when grown under N- } \\
\text { deficient conditions. } \\
2.8 \% \text { increased ALA } \\
\text { in TFA }\end{array}$ & $\begin{array}{l}\text { No significant effect } \\
\text { on growth }\end{array}$ & $\begin{array}{l}\text { (Norashikin } \\
\text { et al., 2018) }\end{array}$ \\
\hline N. oceanica & $\begin{array}{l}\triangle 12-F A D \\
\triangle 9-F A D \\
\triangle 5-F A D\end{array}$ & OE & $\begin{array}{lr}\text { Single and } & \text { co- } \\
\text { expression resulted in } \\
25 \% \text { increased } & \text { EPA. } \\
\text { Decreased } & \text { TFA } \\
\text { content } & \text { in } \\
\text { transformants } & \\
\end{array}$ & $\begin{array}{l}\text { Cell growth increased } \\
\text { in all transformant } \\
\text { lines }\end{array}$ & $\begin{array}{l}\text { (Poliner et } \\
\text { al., 2018) }\end{array}$ \\
\hline
\end{tabular}




\begin{tabular}{|c|c|c|c|c|c|}
\hline P. tricornutum & $\begin{array}{l}\text { PtDGAT2 } \\
\text { OtElo5 }\end{array}$ & $\mathrm{OE}, \mathrm{HE}$ & $\begin{array}{l}\text { Co-expression } \\
\text { resulted in } 37 \text {-fold } \\
\text { increased TAG from } \\
\text { N-replete to starved } \\
\text { conditions, compared } \\
\text { to } 1.8 \text {-fold in wild } \\
\text { type, respectively. } \\
\text { High DHA and DPA } \\
\text { content in TAG }\end{array}$ & $\begin{array}{l}\text { No significant effect } \\
\text { on growth }\end{array}$ & $\begin{array}{l}\text { (Haslam et } \\
\text { al., 2020) }\end{array}$ \\
\hline
\end{tabular}

\subsection{Transcription factors}

Transcription factors (TFs) are DNA-binding proteins that function as key regulators of gene expression. There are several types of TFs that are distinct in their mechanism of recognizing and binding DNA and influencing transcription (Inukai et al. 2017). A single TF can control the expression of multiple genes simultaneously, which makes them an ideal target to shift metabolic fluxes towards lipid biosynthesis. In microalgae, several TFs have been identified as potential targets for genetic engineering, mostly in species of the genera Nannochloropsis and Chlamydomonas.

The first report by Yohn et al. (2011) has shown that overexpression of the chitin binding protein (CBP-like protein SN03) in C. reinhardtii resulted in a $60 \%$ increased TFA content under replete conditions without affecting growth. Tsai et al. (2014) have found the protein "Compromised Hydrolysis of TAG 7" (CHT7) to be a repressor of cellular quiescence in C. reinhardtii. CHT7 knockout caused a differential gene expression under replete conditions, partially resembling that of wild type cells under deplete conditions. Photosynthesis and flagellum assembly-related functions were observed among the regulated genes. Resupply of nutrients in starved $\mathrm{CHT7}$ knockout cells showed a severely hampered remobilization of storage compounds. Although, $\mathrm{CHT7}$ knockout cells did not display an increase in lipid content, regulation of $\mathrm{CHT} 7$ may rewire cellular metabolism under replete conditions.

Ngan et al. (2015) systematically assayed chromatin states and gene expression under nitrogen and sulphur (S) depletion in $C$. reinhardtii. Upregulated expression of "phosphorus starvation response 1" (PSR1) gene showed significant correlation with lipid content during stress condition. PSR1 
had already been investigated before as a regulator of phosphorus metabolism (Wykoff et al., 1999) however, it was not reported to be involved in lipid metabolism. Knockout of PSR1 had no effect on accumulation of TAG under N, $\mathrm{S}$, and $\mathrm{P}$ stress, but PSR1 overexpression increased lipid contents and cell size by $100 \%$ with only minor effects on growth (Ngan et al., 2015). Bajhaiya et al. (2016) have reported that PSR1 knockout in C. reinhardtii caused differential gene expression of 900-1000 genes upon $\mathrm{P}_{\mathrm{i}}$ starvation, most of which are involved in $\mathrm{P}_{\mathrm{i}}$ homeostasis, starch and lipid metabolism, confirming the findings of Ngan et al. (2015). Under deplete conditions, starch and lipid contents decreased in knockout strains by $\sim 60 \%$ and $\sim 70 \%$, respectively. Overexpression of PSR1 in a cell wall-less strain resulted in $\sim 60 \%$ increased starch content and 25\% decreased lipid content. PSR1 seems to be a key regulator of nutrient stress response, however, further investigation is necessary to better understand the effect of this regulator on lipid and starch contents due to the contradictory results.

Recently, Yamaoka et al. (2019) have found that the TF basic leucine zipper 1 (BZIP1) from C. reinhardtii (CrBZIP1) is involved in ER stress response. The protein promotes expression of genes involved in the unfolded protein response. During ER stress, CrBZIP1 knockdown strains showed a reduced expression of genes implicated in DGTS and pinolenic acid biosynthesis, while the expression of the type II DGAT gene DGTT1 was increased. Accordingly, levels of DGTS and pinolenic acid were decreased and TAG content was increased 5.8-9.4-fold compared to the WT.

In recent years, species from the genus Nannochloropsis are emerging as model organisms, shifting TF engineering from the green algae to the heterokonts. The first effort was taken by Kang et al. (2015) who overexpressed a basic helix-loop-helix (bHLH) motif TF in $N$. salina and found a $55 \%$ increased growth rate in the mutant strain with no relevant change in TFA content. While NsbHLH2 overexpression resulted in an overall $43 \%$ increased lipid productivity, growth rates were relatively low compared to values reported in other studies (Poliner et al., 2018; Vieler et al., 2012). 
While the bHLH TF type commonly regulates growth, nutrient uptake and stress response in plants and animals, basic leucine zipper (bZIP) TFs are often involved in abiotic stress, developmental responses and lipid metabolism in plants (Agarwal et al., 2019; Song et al., 2013). Studies have suggested that complex bZIP TFs in plants have evolved from algal ancestors, facilitating the transfer of fundamental knowledge about TF function from plants to microalgae (Corrêa et al., 2008; Peviani et al., 2016). For instance, NsbZIP1 from Nannochloropsis shows homology to type C bZIP TFs present in plants. Moreover, an in silico study based on TF binding sites prediction identified a homologue of NsbZIP1 in N. oceanica as a putative positive regulator of lipid metabolism related genes (Hu et al., 2014). Recently, Kwon and Kang et al. (2018) have achieved the constitutive overexpression of a bZIP TF in N. salina. NsbZIP1 overexpressing strains had increased expression levels of enzymes involved in the Kennedy pathway and fatty acid synthesis causing a simultaneous improvement in growth rate and lipid content. Neutral lipid and TFA contents were increased by $33 \%$ and $21 \%$, respectively, compared to the wild type. Under $\mathrm{N}$ limitation, TFAs and TAGs were further increased by $39 \%$ and $88 \%$, respectively, and cultivation under high salinity stress resulted in $60 \%$ and $203 \%$ higher TFA and TAG contents, respectively, with similar increases in growth rates. A recent study of the NsbZIP1 homologue in $N$. oceanica, NobZIP1, corroborates the importance of this TF (D. W. Li et al., 2019). Although NobZIP1 and the previously described CrbZIP share $73 \%$ sequence identity, they possess very different functions in microalgal metabolism. Consistent with the observations for NsbZIP1, NobZIP1 overexpression resulted in a $65-100 \%$ increase in lipid content and a 60\% decrease in carbohydrate and protein contents without affecting growth or photosynthetic performance. Intriguingly, a substantial part of lipids (up to $40 \%$ on dry cell weight basis) were present in the growth media in mutant cultures (D. W. Li et al., 2019). The authors attribute this finding to thinning of the cell wall in the mutants and hypothesized that this would promote lipid secretion. Overexpression of the TF not only led to an increased expression of $K A S 1, L C-F A C S, A C B P$ and $L P A A T$, but it also decreased the expression of UGDH (encoding UDP-glucose 6-dehydrogenase) which plays a key role in cell wall polymer metabolism. Indeed, $U G D H$ is an attractive target for redirecting 
carbon flux from carbohydrates to lipids (N. N. Li et al., 2017; Oka \& Jigami, 2006), as the authors further found that UGDH knockdown strains displayed the same phenotype regarding cellular composition as NobZIP1 overexpressing strains. Uncertain consequences on the cell wall thinning remain to be investigated. It has been reported that strains with reduced cell wall thickness possess higher mortality rates in industrial scale cultivation system due to shear stress, as previously demonstrated for cell wall-lacking mutants (Barbosa et al., 2003; Chinchin Wang \& Lan, 2018).

Ajjawi et al. (2017) have systematically studied TF expression in $N$. gaditana under $\mathrm{N}$ stress conditions and found 20 putative negative regulators of lipid production. Knock out of a homologue of fungal Zn (II) ${ }_{2} \mathrm{Cys}_{6} \mathrm{TFs}, \mathrm{NgZnCys}$, was found to improve carbon partitioning towards lipids during exponential growth. NgZnCys mutants showed $100-175 \%$ increased TFAs (mainly TAGs), resulting in a significant reduction in growth rate, and thus overall lipid productivity. On the other hand, knockdown of NgZnCys did not affect the growth rate while causing a $100 \%$ increase in TFA content. Cells were substantially bigger, and had $15 \%$ decreased total cellular proteins, whereas carbohydrate content was unaffected. Moreover, $\mathrm{NgZnCys}$ knockdown mutants showed upregulation of six fatty acid desaturases, elongases, lipases and acyltransferases as well as proteins localized on the surface of lipid droplets. However, the relative PUFA contents decreased by $\sim 73 \%$, despite the increase in desaturase expression. The function of the fungal $\mathrm{Zn}$ (II) ${ }_{2} \mathrm{Cys}_{6} \mathrm{TF}$ may be conserved among the genus Nannochloropsis, as $N$. oceanica mutants carrying an insertion cassette in the 3'-UTR of a NgZnCys homologue had an increased neutral lipid content (Südfeld et al., 2021). The authors of this study further found that knockout of an APETALA2-like transcription factor NOAP2 elevated neutral lipid contents and productivity by $\sim 40 \%$ in $N$. oceanica, concomitant with an increased expression of genes related to chloroplastic glycolysis, fatty acid biosynthesis and the Calvin-Benson-Bassham cycle. A different approach was reported by IbáñezSalazar et al. (2014). They investigated the effect of heterologous expression of codon-optimised DNA-binding with one finger type transcription factor (DOFtype TF) GmDOF11 from soybean in C. reinhardtii. Overexpression of this TF induces lipid synthesis in seeds of $A$. thaliana (H.-W. Wang et al., 2007). 
Ibáñez-Salazar et al. (2014) have found that GmDOF11 promotes expression of lipid synthesis-related genes in C. reinhardtii such as BCR1, SQD1 and FAT1. Mutant strains showed a $140 \%$ increase in TFA content during the stationary phase compared to the wild type while the FA profile and growth rate were unaffected. Moreover, Zhang et al. (2014) also showed that heterologous expression of another soybean DOF-type TF GmDOF4 in C. vulgaris resulted in differential expression of 1076 genes in the transgenic strain, 22 of which are related to lipid synthesis. Mixotrophically cultivated mutant strains had a 49$53 \%$ increase in lipids, a 9-14\% decrease in protein and a $15-19 \%$ decrease in carbohydrate content with unchanged growth or fatty acid profile. Another case of heterologous expression of a plant TF was reported for Nannochloropsis salina. Kang et al. (2017) expressed the $A$. thaliana Apetala 2-type transcription factor (AP2-type TF) Wrinkled1 (AtWRI1) which is a master regulator of lipid synthesis during seed maturation. In A. thaliana, AtWRI1 binds to promoter regions (AW-boxes) of several genes involved in lipid biosynthesis related genes (Maeo et al., 2009). In N. salina these promoter regions are present in 475 promoters. Overexpression of AtWRI1 in $N$. salina led to altered regulation of genes including triacylglycerol lipase (TAGL), diacylglycerol kinase (DAGK) and pyruvate phosphate dikinase (PPDK), among others. Growth rates of mutant strains were significantly higher during nitrogen replete conditions, compared to the wild type. Moreover, transformants showed $21-70 \%$ and $32 \%$ increase in TAG and TFA content, respectively, which reportedly resulted in a $64 \%$ higher TFA yield.

TFs can be used as versatile and practical tools for metabolic engineering. Overexpression or knockout of a single TF can lead to up- or downregulation of metabolic pathways or outright rewire the entire cellular metabolism. However, fundamental research needs to be done to elucidate the role of individual TFs in the complex regulatory network of microalgal cells. To this end, predicting relevant TFs with in silico approaches can be an effective way of combining systems biology and metabolic engineering (Hu et al., 2014; Kwon and Kang et al., 2018; D. W. Li et al., 2019). 
Table 4. Genetic engineering of transcription factors for enhanced lipid production

\begin{tabular}{|c|c|c|c|c|c|c|}
\hline Algal Strain & $\begin{array}{l}\text { Target } \\
\text { Genes }\end{array}$ & Strategy & $\begin{array}{l}\text { Regulated } \\
\text { genes }\end{array}$ & $\begin{array}{l}\text { Effect on lipid } \\
\text { synthesis }\end{array}$ & Comments & Ref. \\
\hline C. reinhardtii & $\begin{array}{l}\text { SNO3 } \\
\text { (CREB } \\
\text { binding } \\
\text { Zn-finger } \\
\text { protein) }\end{array}$ & $\mathrm{OE}$ & ND & $\begin{array}{l}60 \% \text { increase in } \\
\text { TFA during } \\
\text { replete }\end{array}$ & $\begin{array}{l}\text { KD impaired in } \\
\text { lipid } \\
\text { accumulation } \\
\text { during deplete }\end{array}$ & $\begin{array}{l}\text { (Yohn et } \\
\text { al., 2011) }\end{array}$ \\
\hline C. reinhardtii & CHT7 & $\mathrm{KO}$ & $\begin{array}{l}2968 \\
\text { differentially } \\
\text { regulated } \\
\text { genes, } \\
\text { enrichment } \\
\text { in flagellum } \\
\text { assembly \& } \\
\text { photosynthe } \\
\text { sis }\end{array}$ & - & $\begin{array}{l}\text { Repressor of } \\
\text { cellular } \\
\text { quiescence, } \\
\text { severely delayed } \\
\text { TAG degradation } \\
\text { upon nutrient } \\
\text { resupply }\end{array}$ & $\begin{array}{l}\text { (Tsai et } \\
\text { al., 2014) }\end{array}$ \\
\hline C. reinhardtii & PSR1 & $\mathrm{OE}$ & ND & $\begin{array}{lr}100 \% & \text { increased } \\
\text { TAG } & \text { under } \\
\text { replete condition }\end{array}$ & $\begin{array}{l}\text { KO strain } \\
\text { significantly } \\
\text { lower TAG during } \\
\text { N, S \& P stress }\end{array}$ & $\begin{array}{l}\text { (Ngan et } \\
\text { al., 2015) }\end{array}$ \\
\hline C. reinhardtii & PSR1 & $\mathrm{OE}$ & $\begin{array}{l}\text { Starch \& } \\
\text { lipid } \\
\text { metabolism } \\
\text { genes under } \\
\text { Pi stress } \\
\end{array}$ & $\begin{array}{l}>50 \% \text { decrease } \\
\text { in lipids during }- \\
P \text { in KOS. } \sim 20 \% \\
\text { decrease in OES }\end{array}$ & $\begin{array}{l}\text { Starch also } \\
\text { decreased in KOS } \\
\text { but increased in } \\
\text { OES }\end{array}$ & $\begin{array}{l}\text { (Bajhaiya } \\
\text { et al., } \\
2016 \text { ) }\end{array}$ \\
\hline C. reinhardtii & CrBZIP1 & $\mathrm{KD}$ & $\begin{array}{l}\text { Pinolenic } \\
\text { acid \& DGTS } \\
\text { biosynthesis }\end{array}$ & $\begin{array}{l}480-860 \% \\
\text { increased TAG, } \\
\text { decreased DGTS } \\
\text { \& pinolenic acid }\end{array}$ & - & $\begin{array}{l}\text { (Yamaoka } \\
\text { et al., } \\
2019)\end{array}$ \\
\hline N. salina & $\mathrm{NsbHLH2}$ & $\mathrm{OE}$ & ND & $\begin{array}{l}43 \% \quad \text { increased } \\
\text { lipid productivity }\end{array}$ & $\begin{array}{l}\text { No change in lipid } \\
\text { content. Growth } \\
\text { rate increased by } \\
55 \%\end{array}$ & $\begin{array}{lr}(\mathrm{N} . & \text { K. } \\
\text { Kang } & \text { et } \\
\text { al., 2015) }\end{array}$ \\
\hline N. salina & NsbZIP1 & $\mathrm{OE}$ & $\begin{array}{l}\text { KAS1, } \quad \text { LC- } \\
\text { FACS, ACBP } \\
\& \angle P A A T\end{array}$ & 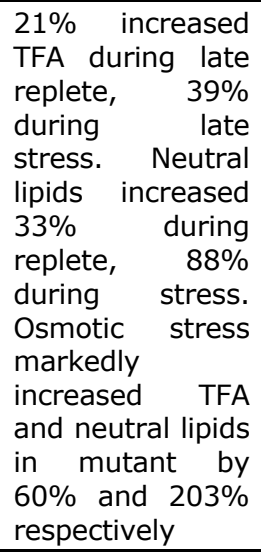 & $\begin{array}{l}\text { Increased QY } \\
\text { growth rate in } \\
\text { mutants } \\
\text { especially under } \\
\text { stress conditions }\end{array}$ & $\begin{array}{l}\text { (Kwon and } \\
\text { Kang et } \\
\text { al., 2018) }\end{array}$ \\
\hline N. oceanica & NobZIP1 & $\mathrm{OE}$ & $\begin{array}{l}\text { Lipid } \\
\text { metabolism } \\
\text { genes } \uparrow \quad \text { (ia. } \\
K A S 1, \quad L C- \\
F A C S, \quad A C B P \\
\& \angle P A A T \text { ) } \\
U G D H \downarrow\end{array}$ & $\begin{array}{lr}65-100 \% & \\
\text { increased lipids } \\
\text { in OE strain. } \\
\text { Further, } 40 \% \\
\text { lipids per dcw } \\
\text { present } \\
\text { medium. in } \\
40 \% \text { decreased } \\
\text { lipid content in } \\
\text { KD strain } \\
\end{array}$ & $\begin{array}{l}\text { Main mode of } \\
\text { action likely } \\
\text { regulation of } \\
U G D H . \quad \text { No } \\
\text { change in growth } \\
\text { rate but thinning } \\
\text { of cell wall }\end{array}$ & $\begin{array}{l}\text { (D. W. Li } \\
\text { et al., } \\
\text { 2019) }\end{array}$ \\
\hline N. gaditana & NgZnCys & $\mathrm{KD}$ & $\begin{array}{l}\text { Desaturases } \\
\text {, elongases, } \\
\text { lipases, } \\
\text { acyltransfer }\end{array}$ & $\begin{array}{l}100 \% \text { increased } \\
\text { TFA during } \\
\text { growth phase in } \\
\text { KD strain }\end{array}$ & $\begin{array}{l}\text { KO strain grows } \\
\text { poorly, KD strain } \\
\text { unaffected in } \\
\text { growth }\end{array}$ & $\begin{array}{l}\text { (Ajjawi et } \\
\text { al., 2017) }\end{array}$ \\
\hline
\end{tabular}




\begin{tabular}{|c|c|c|c|c|c|c|}
\hline & & & $\begin{array}{l}\text { ases \& lipid } \\
\text { droplet } \\
\text { surface } \\
\text { protein } \uparrow \quad \text { in } \\
\text { KD strain }\end{array}$ & $\begin{array}{l}100-175 \% \\
\text { increase in TFA in } \\
\text { KO strain }\end{array}$ & & \\
\hline N. oceanica & NoAP2 & $\mathrm{KO}$ & $\begin{array}{l}\text { Chloroplastic } \\
\text { fatty acid } \\
\text { biosynthesis } \\
\text { glycolysis } \\
\text { and CBB } \\
\text { cycle genes }\end{array}$ & $\begin{array}{lr}40 \% & \text { increased } \\
\text { neutral } & \text { lipids } \\
\text { during } & \text { growth } \\
\text { phase } & \\
\end{array}$ & $\begin{array}{l}\text { No effect on } \\
\text { growth }\end{array}$ & $\begin{array}{l}\text { (Südfeld } \\
\text { et al., } \\
2021)\end{array}$ \\
\hline C. reinhardtii & GmDOF11 & $\mathrm{HE}$ & $\begin{array}{l}\text { Lipid } \\
\text { synthesis } \\
\text { related } \\
(B C R 1 \text {, } \\
S Q D 1 \text { and } \\
\text { FAT1) } \uparrow\end{array}$ & $\begin{array}{lr}140 \% & \text { increased } \\
\text { TFA } & \text { during } \\
\text { stationary phase }\end{array}$ & $\begin{array}{l}\text { No effect on } \\
\text { growth }\end{array}$ & $\begin{array}{l}\text { (Ibáñez- } \\
\text { Salazar et } \\
\text { al., 2014) }\end{array}$ \\
\hline $\begin{array}{l}\text { Chlorella } \\
\text { ellipsoidea }\end{array}$ & GmDOF4 & $\mathrm{HE}$ & $\begin{array}{l}1076 \text { genes } \\
\text { differentially } \\
\text { expressed in } \\
\text { mutant. } 22 \\
\text { genes } \\
\text { related to } \\
\text { lipid } \\
\text { synthesis } \\
\text { (i.e. } \\
\text { ACCase } \uparrow \text { ) }\end{array}$ & $\begin{array}{ll}49-53 \% & \\
\text { increased } & \text { lipids } \\
\text { during } & \text { early } \\
\text { stationary } & \text { phase }\end{array}$ & $\begin{array}{l}\text { No effect on } \\
\text { growth. } \\
\text { Decreased } \\
\text { carbohydrate } \\
\text { and protein in } \\
\text { mutant }\end{array}$ & $\begin{array}{l}\text { (J. Zhang } \\
\text { et al., } \\
2014)\end{array}$ \\
\hline N. salina & AtWRI1 & $\mathrm{HE}$ & $\begin{array}{lr}\text { i.e. } & T A G L, \\
D A G K & \text { and } \\
P P D K \uparrow & \end{array}$ & $\begin{array}{lr}32 \% & \text { increased } \\
\text { TFA, } & 21-70 \% \\
\text { increased } & \text { TAG } \\
\text { during } & \text { replete } \\
\text { phase. } & 64 \% \\
\text { higher TFA yield }\end{array}$ & $\begin{array}{l}\text { Increased growth } \\
\text { rate, especially } \\
\text { under N } \\
\text { limitation, but no } \\
\text { increase in TFA } \\
\text { during }-\mathrm{N} \text {. }\end{array}$ & $\begin{array}{lr}\text { (N. } & \text { K. } \\
\text { Kang et } & \text { et } \\
\text { al., 2017) }\end{array}$ \\
\hline
\end{tabular}

\subsection{Photosynthesis and NADPH generation}

\subsubsection{Photosynthetic electron transport, redox homeostasis and energy balancing.}

In eukaryotic microalgae, photosynthesis occurs in the thylakoid membranes of the chloroplast. A highly complex and conserved mechanism transfers electrons from $\mathrm{H}_{2} \mathrm{O}$ to $\mathrm{NADP}^{+}$in a series of redox reactions from redox couples with lower potentials to those with higher potentials. Such reactions are driven by light energy and take place in chlorophylls P680 in the photosystem II (PSII) reaction center and P700 in photosystem I (PSI). The electron transfer from PSII to PSI is coupled to $\mathrm{H}^{+}$transfer from stroma to lumen, resulting in a $\mathrm{H}^{+}$ gradient across the thylakoid membrane, facilitating ATP synthesis by an ATP synthase (ATPase). A part of the energy and reducing equivalents produced in light reactions are subsequently consumed in the Calvin-Benson-Bassham 
(CBB) cycle, which converts $\mathrm{CO}_{2}$ into glyceraldehyde 3-phosphate (GAP). GAP can follow different routes and can be converted into sugars (gluconeogenesis) or pyruvate and subsequently acetyl-CoA, which provides energy to the cells for lipid formation, maintenance, anabolism and growth (Subramanian et al., 2013). Because lipid synthesis usually occurs in the chloroplast, it is influenced by light conditions (Lehmuskero et al., 2018; Masojídek et al., 2013; Sato \& Moriyama, 2018), carbon flux from GAP to acetyl-CoA and involvement of other cellular compartments. Recent studies have improved carbon flux towards lipid synthesis in microalgae either by improving photosynthetic capacity or by increasing the supply of reducing equivalents via genetic engineering. In this section we review relevant advances in both genetic strategies that lead to improved lipid contents.

\subsubsection{Engineering photosynthetic performance for enhanced lipid production}

Improving light reactions was attempted by overexpressing ferredoxin 1 (PETF) and 5 (FDX5) in C. reinhardtii (Huang et al. 2015). PETF is involved in electron transfer from PSI to $\mathrm{NADP}^{+}$through ferredoxin $\mathrm{NADP}^{+}$oxidoreductase (FNR) and FDX5 and it has been linked to reactive oxygen species (ROS) detoxification. Moreover, FDX5 was shown to physically interact with the fatty acid desaturases CrA4FAD and CrFAD6, likely donating electrons for the desaturation of fatty acids that stabilize monogalactosyldiacylglycerol (W. Yang et al., 2015). Separate overexpression of both proteins increased resistance to heat, salt stress, lowered intracellular levels of $\mathrm{H}_{2} \mathrm{O}_{2}$ and increased lipid contents by $13-56 \%$ and $50-250 \%$ under replete and deplete conditions, respectively. Furthermore, promising results were also obtained when overexpressing a ROS stress-response related enzyme superoxide dismutase (SOD1) in Schizochytrium sp. (S. Zhang et al., 2018). SOD1 overexpression not only increased ROS detoxification but also enhanced TFA accumulation without affecting biomass yields. An $18 \%$ and $37 \%$ increase in SFA and PUFA contents were observed, respectively. Since PUFAs have antioxidant properties (X. Y. Liu et al., 2013; Richard et al., 2008; Schmid-Siegert et al., 2016), higher levels of SOD1-mediated ROS scavenging may have caused a decreased 
depletion of PUFA pools via alleviation of lipid peroxidation, although further investigation is necessary.

Gargouri et al. (2017) observed that expression levels of PSI translation initiation factor $T A B 2$ in $C$. reinhardtii are markedly increased during early nutrient stress conditions. A TAB2 knockout strain showed lower levels of PSI and significantly increased starch $(>800 \%)$ and TAG $(>100 \%)$ contents compared to WT strains under replete condition and mixotrophic cultivation. Additionally, TAB2 knockouts showed lower NADPH/NADP ${ }^{+}$and ATP/NADPH ratios under replete and deplete conditions, respectively. These results suggest that carbon partitioning to carbohydrates and lipids is regulated by PSI activity and it is likely linked to ATP and NADPH generation in green algae. Furthermore, Koh et al. (2019) expressed the heterologous chlorophyllide an oxygenase gene from $C$. reinhardtii ( $C r C A O)$ in $N$. salina strains. CrCAO overexpressing strains showed significant contents of chlorophyll a (Chl a), chlorophyll b (Chl b), lightharvesting complex II (LHCII) antenna proteins and PSII reaction center protein D1, leading to a slight increase in photosynthetic performance under higher light conditions. Under lower light conditions an increased growth rate was observed leading to an overall increase in lipid productivity of $\sim 42 \%$ compared to the wild type.

\subsubsection{Engineering NADPH generation for enhanced lipid production}

Promising research has been directed towards increasing levels of reducing equivalents in order to boost FA synthesis in microalgae. Potential targets are malic enzymes (ME) which are $N A D(P)^{+}$-dependent oxidoreductases that perform the oxidative decarboxylation of malate to pyruvate (Cornish-Bowden, 2014). In plants and microalgae, different types of MEs have preference for either $\mathrm{NAD}^{+}$, $\mathrm{NADP}^{+}$or both. In C4-plants, they are localized in the chloroplast and participate in carbon-concentrating mechanisms to increase efficiency of RuBisCO activity (Gerald E \& Carlos S, 1992; Madhavan et al., 2002). On the other hand, cytosolic isoforms involved in redox homeostasis and energy metabolism supply reducing power to biosynthetic pathways and generate 
pyruvate for ATP production in the mitochondria (Drincovich et al., 2001). FA synthesis requires substantial NADPH supply and it was suggested to be the limiting factor for lipid synthesis in oleaginous organisms (Ratledge, 2014; Wynn et al., 1999). Talebi et al. (2014) have reported for the first time the expression of ME in the microalga Dunaliella salina. Successful simultaneous overexpression of the putative cytosolic $\mathrm{NADP}^{+}$-dependent malic enzyme DSME1 and acetyl CoA-carboxylase subunit D DsAccD was achieved in the chloroplast. Mutant strains showed a $12 \%$ and $23 \%$ increase in TFAs and neutral lipids, respectively, and the FA profile was substantially shifted towards saturated fatty acids at the expense of unsaturated ones. A putative mitochondrial ME was overexpressed in P. tricornutum by Xue et al. (2015). Overexpression of PtME resulted in $150 \%$ increase in TFA content reaching $\sim 58 \% \mathrm{FA} \mathrm{dcw}^{-1}$ with a slight negative affect on growth rates. The authors also transferred the mitochondrial-localized PtME to Chlorella pyrenoidosa (Xue et al., 2016). They report a $>300 \%$ increase in ME activity in cell extracts along with $220 \%$ increase in TFAs under replete conditions and $360 \%$ increase under deplete conditions, reaching $40.9 \%$ and $58.7 \%$ dcw $^{-1}$ respectively. Zhu et al. (2018) also investigated the cytosolic ME expression in P. tricornutum. PtME1 overexpression led to a $52-81 \%$ increase in $\mathrm{NADP}^{+}-\mathrm{ME}$ activity in cell extracts and $28.4-80.3 \%$ increase in intracellular NADPH levels, resulting in $150 \%$ increase in TFA content (57.8\% dcw ${ }^{-1}$ during non-stressed conditions). The MUFA content was decreased from $\sim 25$ to $\sim 20 \%$, while the SFAs and PUFAs contents showed a slight increase from $\sim 23$ to $\sim 25 \%$ and from 49 to $54 \%$, respectively.

Another source of cellular reducing power is the oxidative pentose phosphate pathway (oxPPP) (Kruger \& von Schaewen, 2003). The oxPPP provides metabolic intermediates such as ribose-5-phosphate and generates up to 12 NADPH molecules per molecule of G6P. However, the oxPPP involves an oxidative decarboxylation step leading to $\mathrm{CO}_{2}$ production resulting in lower triose phosphate yields compared to other glycolytic pathways. While oxPPP lowers the lipid yield on substrate in heterotrophic processes, photoautotrophic cultivation may not be limited as $\mathrm{CO}_{2}$ can be re-assimilated. Enzymes in the oxPPP are present not only in the cytosol but also in chloroplasts, indicating 
close spatial proximity of decarboxylation reactions and carbon fixation mechanisms (Kruger \& von Schaewen, 2003). The plastidic isoforms of these enzymes are redox-regulated and have been proposed to supply NADPH in absence of photosynthesis (Hauschild \& von Schaewen, 2003). The ratelimiting step of the oxPPP is the conversion of G6P to 6-phosphogluconolactone coupled to the reduction of $\mathrm{NADP}^{+}$, which is catalyzed by G6P dehydrogenase (G6PD). Xue et al. (2017) characterized G6PD in $P$. tricornutum. Overexpression of the plastidic PtG6PD resulted in a $170 \%$ increase in TFA levels $\left(55.7 \% \mathrm{dcw}^{-1}\right)$ without affecting growth of mutant strains. The SFA and MUFA contents increased while the PUFA content decreased. Moreover, metabolomic analyses revealed elevated levels of glycolytic and TCA cycle intermediates such as pyruvate and acetyl-CoA, which were increased by $300 \%$ and $710 \%$, respectively. Additionally, 3-phosphoglycerate was elevated by $570 \%$, suggesting a possible increase of FA synthesis. Furthermore, the heterologous expression of NoG6PD from $N$. oceanica in C. pyrenoidosa (Xue et al., 2020) increased NADPH and TAG levels by $119 \%$ and $209 \%$ during both growth and stationary phases. In this study, the TFA content was increased by 230-260\% under nitrogen deplete conditions, with an increase in MUFA and PUFA contents at the expense of SFAs. Carbohydrates and protein levels were drastically reduced in the engineered strains without negatively impacting the growth rate.

Fan et al. (2015) observed 40-80\% increased NADPH levels in C. pyrenoidosa strains expressing an NAD kinase enzyme from $A$. thaliana (AtNADK3). This resulted in a $45-110 \%$ increase in TFA content under heterotrophic and mixotrophic cultivation conditions.

Alternatively, increasing cellular reducing power towards microalgal lipid biosynthesis can be achieved by expressing, for instance, transhydrogenase enzymes. This has been attempted and reported in E. coli (He et al., 2014). Soluble transhydrogenase transfers electrons from NADPH to $\mathrm{NAD}^{+}$and membrane-bound isoforms transfer electrons from NADH to NADP ${ }^{+}$driven by a proton gradient to overcome high NADPH/NADH ratios in prokaryotes and in eukaryotic mitochondria (Sauer et al., 2004). Overexpression of the 
membrane-bound isoform or knockdown/knockout of soluble transhydrogenase might increase NADPH/NADH ratios and FA synthesis in microalgae. Another enzyme involved in generating cellular reducing power is the $\mathrm{NADP}^{+}$-dependent isocitrate dehydrogenase (IDH) which has been reported to be the main source for NADPH required for fatty acid synthesis in mammalian adipocytes (H.-J. Koh et al., 2004; S. M. Lee et al., 2002; Shechter et al., 2003). Cytosolic and chloroplastic IDH are present in microalgae but have thus far not been given attention as targets for increasing NADPH production rates (Martínez-Rivas \& Vega, 1994).

Table 5. Genetic engineering of $N A D P H$ generation for enhanced lipid production

\begin{tabular}{|c|c|c|c|c|c|}
\hline Algal Strain & $\begin{array}{l}\text { Targeted } \\
\text { Gene (s) }\end{array}$ & Strategy & $\begin{array}{l}\text { Effect on lipid } \\
\text { synthesis }\end{array}$ & Comments & References \\
\hline N. oceanica & $\mathrm{nRca}$ & $\mathrm{OE}$ & - & $\begin{array}{l}\text { 30\% increased QY and } \\
\text { growth }>\quad 30 \% \\
\text { increased } \\
\text { productivity }\end{array}$ & $\begin{array}{l}\text { (L. Wei et al., } \\
\text { 2017) }\end{array}$ \\
\hline C. reinhardtii & $\begin{array}{l}\text { PETC } \\
\text { FDX5 }\end{array}$ & $\mathrm{OE}$ & $\begin{array}{l}50-250 \% \text { increased } \\
\text { lipid content during } \\
\text { deplete for both } \\
\text { proteins }\end{array}$ & $\begin{array}{l}110-170 \% \quad \text { increased } \\
\text { starch concomitantly }\end{array}$ & $\begin{array}{l}\text { (L.-F. Huang } \\
\text { et al., 2015) }\end{array}$ \\
\hline $\begin{array}{l}\text { Schizochytrium } \\
\text { sp. }\end{array}$ & SOD1 & $\mathrm{OE}$ & $\begin{array}{l}18 \% \text { increased SFA, } \\
37 \% \text { increased PUFA }\end{array}$ & Lower levels of ROS & $\begin{array}{l}\text { (S. Zhang et } \\
\text { al., 2018) }\end{array}$ \\
\hline C. reinhardtii & TAB2 & $\mathrm{KO}$ & $\begin{array}{lr}>100 \% & \text { increased } \\
\text { TAG } & \text { during } \\
\text { mixotrophic } & \text { replete } \\
\text { cultivation } & \end{array}$ & $\begin{array}{l}>800 \% \text { increased } \\
\text { starch levels. Energy } \\
\text { metabolism severely } \\
\text { impaired, higher cell } \\
\text { mortality under -N }\end{array}$ & $\begin{array}{l}\text { (Gargouri et } \\
\text { al., 2017) }\end{array}$ \\
\hline N. salina & CrCAO & $\mathrm{HE}$ & $\begin{array}{l}42 \% \text { increased } \\
\text { productivity due to } \\
\text { increased growth } \\
\text { under light-limited } \\
\text { conditions }\end{array}$ & & $\begin{array}{l}\text { (H. G. Koh et } \\
\text { al., 2019) }\end{array}$ \\
\hline D. salina & $\begin{array}{l}\text { AccD } \\
M E\end{array}$ & $\mathrm{OE}$ & $\begin{array}{l}12 \% \text { increased TFA, } \\
23 \% \text { increased NL }\end{array}$ & & $\begin{array}{l}\text { (Talebi et al., } \\
\text { 2014) }\end{array}$ \\
\hline P. tricornutum & ME & & $\begin{array}{l}150 \% \text { increased } \mathrm{NL} \\
\text { during replete, } 66 \% \\
\text { increased NL during } \\
\text { deplete }\end{array}$ & & $\begin{array}{l}\text { (Xue et al., } \\
2015)\end{array}$ \\
\hline C. pyrenoidosa & PtME & $\mathrm{HE}$ & $\begin{array}{l}220 \% \text { increased TFA } \\
\text { during replete, } \\
360 \% \text { during deplete }\end{array}$ & & $\begin{array}{l}\text { (Xue et al., } \\
2016)\end{array}$ \\
\hline P. tricornutum & ME & $\mathrm{OE}$ & $\begin{array}{l}150 \% \text { increased TFA } \\
\text { during replete }\end{array}$ & Decreased MUFA & $\begin{array}{l}\text { (B.-H. Zhu et } \\
\text { al., 2018) }\end{array}$ \\
\hline P. tricornutum & G6PD & $\mathrm{OE}$ & $\begin{array}{l}170 \% \text { increased } \\
\text { total lipids during } \\
\text { replete phase, } 100 \% \\
\text { during deplete }\end{array}$ & & $\begin{array}{l}\text { (Xue et al., } \\
2017)\end{array}$ \\
\hline C. pyrenoidosa & NoG6PD & $\mathrm{HE}$ & $\begin{array}{l}230-260 \% \text { increased } \\
\text { TFA during } \\
\text { starvation phase. } \\
209 \% \text { increase in } \\
\text { TAGs }\end{array}$ & $\begin{array}{l}119 \% \quad \text { increased } \\
\text { intracellular NADPH }\end{array}$ & $\begin{array}{l}\text { (Xue et al., } \\
2020)\end{array}$ \\
\hline
\end{tabular}




$\begin{array}{lllll}\text { C. } \text { pyrenoidosa } & \text { AtNADK3 } & \text { HE } & \begin{array}{l}45-110 \% \text { increased } \\ \text { total lipids during }\end{array} & \text { (Fan et al., }\end{array}$

growth

\subsection{Central carbon metabolism}

The central carbon metabolism plays a fundamental role in directing carbon fluxes to different metabolic pathways, and it regulates inter alia acetyl-CoA and G3P availability (Subramanian et al., 2013). For instance, G3P can function as substrate for TAG biosynthesis, but it can also be reconverted to DHAP and enter glycolysis or gluconeogenesis (Han et al., 2016). In this context, Muto et al. (2015) attempted to alter glycerol availability by overexpression of an endogenous glycerol kinase (GK) gene in the oleaginous diatom Fistulifera solaris. Although only $12 \%$ increase in lipid yield was observed, externally supplied glycerol was utilized $40 \%$ more efficiently in overexpression strains resulting in a slight increase in biomass productivity.

Isotopic labelling studies in higher plants have shown that increased levels of intracellular G3P correlate with carbon partitioning of acetyl-CoA to TAG (Vigeolas \& Geigenberger, 2004). These findings support the idea that G3P levels may be limiting for TAG synthesis only under conditions of increased acyl-CoA availability. It was also found that overexpression of G3P dehydrogenase (G3PDH) in P. tricornutum led to a $580 \%$ increase in intracellular glycerol levels and improvement of lipid content by $60 \%$ during the stationary phase (Yao et al., 2014). The growth rates of mutant strains were lower compared to the wild type and the neutral lipid content was elevated by $90 \%$ with an increase in MUFA content at the expense of PUFAs. These findings have shown that G3P is a limiting substrate for glycerolipid synthesis in $P$. tricornutum under nutrient stress conditions thereby emphasizing the importance of G3PDH as a link between glycolysis and glycerol metabolism.

Furthermore, the connection between glycolytic flux and lipid production was investigated in the marine diatom Thalassiosira pseudonana (Abbriano et al., 2018). Overexpression of the glycolytic regulator 6-phosphofructo-2kinase/fructose-2,6-bisphosphatase (PFK2/F2BP) enhanced the flux through 
the glycolytic pathway, resulting in increased production of neutral lipids by $116 \%$ and proteins by $35 \%$ at the expense of storage carbohydrates under both stressed and non-stressed conditions. Engineered strains showed a delayed progression through the cell cycle ( $\mathrm{G} 1$ to the S phase), resulting in an overall decrease in growth rate. Hypothetically, this may be the effect of a decreased availability of glucose for the PPP and consequently less synthesis of C5 sugars required for nucleotide synthesis. Although diatoms are complex study organisms due to the presence of glycolytic pathways in the cytosol, mitochondria and plastid, the results from Abbriano and co-workers highlight the potential of redirecting the carbon flux by modifying glycolytic pathways.

Table 6. Genetic engineering of central carbon metabolism for enhanced lipid production

\begin{tabular}{|c|c|c|c|c|c|}
\hline Algal Strain & $\begin{array}{l}\text { Targeted } \\
\text { Gene (s) }\end{array}$ & Strategy & $\begin{array}{l}\text { Effect on lipid } \\
\text { synthesis }\end{array}$ & Comments & References \\
\hline F. solaris & GK & $\mathrm{OE}$ & $\begin{array}{l}12 \% \text { increase in lipid } \\
\text { yield }\end{array}$ & $\begin{array}{l}\text { Glycerol usage up } \\
40 \% \text { in mixotrophic } \\
\text { cultivation. No } \\
\text { analysis done during } \\
\text { stationary phase }\end{array}$ & $\begin{array}{l}\text { (Muto et al., } \\
\text { 2015) }\end{array}$ \\
\hline P. tricornutum & G3PDH & $\mathrm{OE}$ & $\begin{array}{l}60 \% \text { increased total } \\
\text { lipids, } 90 \% \text { increased } \\
\text { neutral lipids during } \\
\text { stationary phase }\end{array}$ & $\begin{array}{l}20 \% \text { reduced cell } \\
\text { concentration }\end{array}$ & $\begin{array}{l}\text { (Yao et al., } \\
2014)\end{array}$ \\
\hline T. pseudonana & PFK2/F2BP & & $\begin{array}{l}116 \% \text { increased TAG } \\
\text { under growth and } \\
\text { starvation conditions }\end{array}$ & $\begin{array}{l}\text { Cell cycle progression } \\
\text { slowed, lower growth } \\
\text { rate. }\end{array}$ & $\begin{array}{l}\text { (Abbriano et } \\
\text { al., 2018) }\end{array}$ \\
\hline
\end{tabular}

\subsection{Other approaches}

\subsubsection{Lipid catabolism}

In higher eukaryotes, TAG is stored in multi-functional cytosolic lipid droplets (LDs) (A. H. C. Huang, 1992; Olzmann \& Carvalho, 2019; Thiam \& Beller, 2017; C. Zhang \& Liu, 2017). Although TAG assembly and LD formation have been reported for some microalgae to occur also in the chloroplast, the subject is still under debate (Balamurugan et al., 2017; Eugeni Piller et al., 2011; Fan et al., 2011; Moriyama et al., 2018). While the mechanism involved in LD formation remains poorly understood, it has been reported that LDs bud from the ER 
membrane towards the cytosol with the aid of specialized proteins (Jacquier et al., 2013; Morris \& Olzmann, 2019). LDs are composed of a hydrophobic core, consisting mostly of TAG and enclosed by a phospholipid monolayer with a broad range of proteins. These proteins can be involved in RNA binding, translation (C. Zhang \& Liu, 2017), membrane trafficking (Olzmann \& Carvalho, 2019), signal integration, lipogenesis and lipolysis (Kong et al., 2018). Moreover, several components involved in these processes have been identified in Chlamydomonas reinhardtii such as CXC-domain containing regulatory protein, phosphatidylethanolamine-binding delayed in TAG hydrolysis-1 (DTH1) (Lee et al., 2020), two lipases and two enzymes involved in FA $\beta$-oxidation (LiBeisson et al., 2021). However, the most well-characterized function is that of oleosins and perilipins families which prevent LD coalescence.

The Stramenopile lineage-specific LD protein StLDP was identified in $P$. tricornutum as a major class of LD coat proteins. Recently, Yoneda et al. (2018) have reported that StLDP overexpression in $P$. tricornutum leads to an increased number of LDs and $\sim 25 \%$ higher neutral lipid content during late stage of nutrient starvation. The authors hypothesized that StLDP sequesters LDs during LD formation and prevents LD coalescence during nitrogen starvation. A high number of lipid droplets implies a high surface to volume ratio of individual LDs, thereby increasing the available area for lipogenic and lipolytic coat proteins such as DGAT2 and lipases, resulting i.a. in increased remobilization capacity of stored TAGs (Thiam \& Beller, 2017).

Patatin-like phospholipase domain-containing protein 3 (PNPLA3) is a LD membrane protein identified in animal cells which has a controversial biological function (Kienesberger et al., 2009). An amino acid substitution in mammalian PNPLA3 causes fatty liver disease (Kienesberger et al., 2009) and expression of the gene encoding catalytically inactive PNPLA ${ }^{\mathrm{I} 148 \mathrm{M}}$ in mice causes increased hepatic TAG content (J. Z. Li et al., 2012). On the other hand, in yeast, human PNPLA3 drives TAG hydrolysis (Pingitore et al., 2014) while murine PNPLA3 exhibits LPAT activity in vitro (Kumari et al., 2012). Wang et al., 2015 identified and characterized a PNPLA3 ortholog in P. tricornutum. PtPNPLA3overexpressing strains showed a $55 \%$ increase in neutral lipid content and a 
$26 \%$ increase in PUFA content (mainly C20:4), during stationary phase. The protein shows homology to the catalytic domain of a cytosolic phospholipase A2 which hydrolyzes the sn-2-acyl ester bonds of C20:4 FAs from phospholipids. Accordingly, PtPNPLA3 may have phospholipase activity and thereby increase the availability of C20:4 FAs for CoA esterification and incorporation into TAG. Similar results were observed when expressing the heterologous human HSPNPLA $3^{I 148 M}$ in P. tricornutum. Results showed a $64 \%$ increase in TAG content and $52.5 \%$ increased TFA dcw ${ }^{-1}$ during the transition to the stationary phase (X. Wang, Wei, et al., 2018). Whereas PtPNPLA3 was proposed to exhibit phospholipase activity, which increases FA availability for TAG synthesis, phospholipid diacylglycerol acyl transferases (PDATs) catalyze the direct transfer of an acyl group from lipid donors like membrane phospholipids to DAG (Dahlqvist et al., 2000; Yoon et al., 2012). Heterologous expression of PDAT from S. cerevisiae led to $22 \%$ and $32 \%$ increase in TFA and TAG content in $C$. reinhardtii during growth and early stationary phases when targeted to the chloroplast (Z. Zhu et al., 2018). A substantial increase in PUFAs was observed whereas MGDG content was lower in the mutant strain. Presumably, the transfer of acyl groups from PUFA-rich MGDGs to TAGs may enhance PUFA synthesis in order to replenish photosynthetically active compounds in the thylakoid membrane MGDGs. Although photosynthetic performance and growth rates were lower, final biomass densities were similar to the WT. PDAT overexpression seems to be a suitable approach to change FA distribution and lipid profiles in microalgae cells, though compromising the chloroplast integrity.

\subsubsection{Others}

The protein UBC2 is involved in Lys63-linked polyubiquitination in C. reinhardtii (Fei et al., 2017). This type of polyubiquitination is a regulatory mechanism unlike the Lys-48-linked polyubiquitination, which is a key step in proteolysis (Volk et al., 2005). In C. reinhardtii it has been proposed that UBC2 functions in DNA damage tolerance. However, Fei et al. (2017) have found that in UBC2 knockdown mutants the neutral lipid content of cells was $13.5-35.2 \%$ lower than in wild type. UBC2 overexpression strains showed a $100 \%$ increase in 
neutral lipids with no impact on growth rate. These findings suggest that UBC2 is involved in other cellular processes than DNA damage response in $C$. reinhardtii. Accordingly, similar observations were reported for a homologous protein UEV1A in $A$. thaliana (Wen et al., 2014).

Lastly, several studies have proposed that fatty acid content could be increased by shunting carbon precursors from starch by blocking or reducing starch biosynthesis in the cells. Starchless mutants of Scenedesmus obliquus, Chlorella pyrenoidosa and Chlamydomonas reinhardtii generated using ultraviolet radiation as a mutagen showed increased FA content (de Jaeger et al., 2014; Li et al., 2010; Ramazanov et al., 2006). The starchless mutant of S. obliquus showed an increase in TFA productivity of $41 \%$ after nitrogen depletion, reaching $49.4 \%$ of TAGs (\% DW), without a significantly decreased biomass productivity (de Jaeger et al., 2014). Starchless mutant of $C$. pyrenoidosa showed an increase of PUFA content by $20.4 \%$ and decrease of saturated FA by $18 \%$ compared to WT strains. In addition, TFAs were increased from 25 to $38 \%$ (\% DW) under nitrogen limitation and mutants presented higher growth rates and productivities compared to WT cells during nitrogen replete conditions (Ramazanov et al., 2006). Moreover, the inactivation of an ADP-glucose pyrophosphorylase via UV mutagenesis in C. reinhardtii generated a starchless mutant with a 10-fold increase in TAG content and a TFA content of $47 \%$, up from $13 \%$ for the WT strain. However, this resulted in $30 \%$ decrease in growth compared to the WT strain under autotrophic conditions (Li et al., 2010). Conversely, this approach did not lead to significant increase in lipid accumulation or change in fatty acid profile in lipids in the starchless mutant of Chlorella sorokiniana compared to WT (Vonlanthen et al., 2015).

Table 7. Other approaches for enhanced lipid production

\begin{tabular}{|c|c|c|c|c|c|}
\hline Algal Strain & $\begin{array}{l}\text { Targeted } \\
\text { Gene }\end{array}$ & Strategy & $\begin{array}{l}\text { Effect on } \\
\text { synthesis }\end{array}$ & Comments & References \\
\hline P. tricornutum & StLDP & OE & $\begin{array}{l}25 \% \text { increased TAG } \\
\text { during late starvation }\end{array}$ & $\begin{array}{l}\text { No expression of } \\
\text { transgene during } \\
\text { starvation phase }\end{array}$ & $\begin{array}{l}\text { (Yoneda et } \\
\text { al., 2018) }\end{array}$ \\
\hline P. tricornutum & PNPLA3 & OE & $55 \%$ increased TAG & $26 \%$ increase in PUFA & $\begin{array}{l}\text { (X. Wang et } \\
\text { al., 2015) }\end{array}$ \\
\hline P. tricornutum & HsPNPLA3 & $\mathrm{HE}$ & $\begin{array}{l}52 \% \text { increased TFA, } \\
64 \% \text { increased TAG }\end{array}$ & $\begin{array}{l}\text { Increased } \\
\text { decreased } \\
\text { levels }\end{array}$ & $\begin{array}{l}\text { (X. Wang, } \\
\text { Wei, et al., } \\
2018)\end{array}$ \\
\hline
\end{tabular}




\begin{tabular}{|c|c|c|c|c|c|}
\hline & & & $\begin{array}{l}\text { during late log/early } \\
\text { stationary }\end{array}$ & & \\
\hline C. reinhardtii & ScPDAT & $\mathrm{HE}$ & $\begin{array}{l}22 \% \text { increased TFA, } \\
32 \% \text { increased TAG } \\
\text { during growth }\end{array}$ & $\begin{array}{lr}\text { Delayed } & \text { growth, } \\
\text { substantial } & \begin{array}{r}\text { increase } \\
\text { in PUFAs, lower }\end{array} \\
\text { MGDG } & \end{array}$ & $\begin{array}{l}\text { (Z. Zhu et } \\
\text { al., 2018) }\end{array}$ \\
\hline C. reinhardtii & UBC2 & OE & $\begin{array}{lr}\sim 100 \% & \text { higher TAG } \\
\text { during } & \text { stationary } \\
\text { phase } & \end{array}$ & $\begin{array}{l}\text { No impact of OE on } \\
\text { growth. KD strains } \\
13-35 \% \text { lower in TAG }\end{array}$ & $\begin{array}{l}\text { (Fei et al., } \\
2017 \text { ) }\end{array}$ \\
\hline
\end{tabular}

\section{Conclusion and future perspectives}

The ability of microalgae to grow photo-autotrophically on non-arable land makes them a suitable platform for green chemical production. However, the high cost of biomass production has hampered the commercialization of various microalgal products including third generation biofuels (Ruiz et al., 2016). Genetic engineering of microalgae has been seen as a solution to this bottleneck. This review summarizes the various advancements in genetic engineering of microalgae with improved lipid productivities. Most of the discussed engineering strategies involve modifications of a single metabolic pathway with the aim to either channel carbon towards lipid synthesis or to improve carbon capture efficiency. Even though several approaches have improved lipid accumulation in transgenic strains, productivities remain too low for economically feasible production of biofuels from microalgae (Ajjawi et al., 2017; Remmers et al., 2018).To further improve lipid productivities, engineering strategies will have to simultaneously improve the photosynthesis reactions and channel the carbon flux towards lipids without limiting the growth in host species. Proposing detailed approaches to improve the microalgal productivities is outside the scope of this review. Nevertheless, a recent review has presented various synthetic biology approaches that could potentially improve the productivities of microalgae (Naduthodi et al., 2021).

Evidently, substantial advancements have been achieved in the field of microalgal biotechnology for sustainable production of third generation biofuels and green chemicals. Bioprospecting of more suitable host species and/or metabolic engineering of genetically accessible microalgal strains could accomplish the aim of commercially feasible lipid production. Future research 
may need to parallelly focus on microalgae that are the most promising to industrially produce high-value lipids, as these are most likely to reach economic feasibility on the short term. The heterokont genus Nannochloropsis has already received considerable attention and it is a promising candidate for EPA production. More recently, the heterokont Schizochytrium, and the haptophyte Tisochrysis are emerging as potential lipid production platforms due to high growth rates, as well as high natural EPA and DHA contents, respectively (Barten et al., 2020). However, the light harvesting and carbon capturing systems of fast-growing species need to be investigated to allow for the development of genetic engineering strategies aiming to increase biomass and lipid productivities.

\section{Declaration of interest}

The authors declare that they have no conflict of interest.

\section{Acknowledgements}

A part of this work was funded by the Netherlands Organization of Scientific Research (NWO) Building Blocks of Life programme (grant number 737.016.007), and by the Bio-Based Industries Joint Undertaking under the European Union's Horizon 2020 research and innovation program MAGNIFICENT (grant number 745754). 



\section{Chapter 6}

General discussion 


\section{General discussion}

Microalgae are unicellular eukaryotic photosynthetic microorganisms that have attracted the attention of the scientific community due to their ability to convert sunlight, water, $\mathrm{CO}_{2}$ and minor other nutrients into numerous valuable products (Chisti, 2007; Christaki et al., 2011; Draaisma et al., 2013; Gimpel et al., 2013; Pulz \& Gross, 2004; Wijffels et al., 2013; Wijffels \& Barbosa, 2010). They perform these conversions at relatively high growth rates and product yields compared to those achieved with agricultural crops, without the need of arable lands. Moreover, unlike higher plants, some microalgal species can be grown on seawater or wastewater (Brennan \& Owende, 2010; Griffiths \& Harrison, 2009; Medipally et al., 2015; Remmers et al., 2018) in addition to freshwater. Thus, microalgae can be used as a sustainable biological platform to mitigate anthropogenic environmental impact of traditional industrial processes by limiting the use of natural resources, reducing greenhouse gas emissions and being used as feedstock to combat increasing global demand for food, feed and fossil-based products.

Microalgal biomass can be used as a source of numerous industrially relevant compounds including antioxidants, carotenoids, proteins, polysaccharides, polyunsaturated fatty acids, triacylglycerols (TAG), sterols and vitamins (Chisti, 2007; Christaki et al., 2011; Draaisma et al., 2013; Pulz \& Gross, 2004). However, development of a cost-effective production process requires the optimization of cultivation systems and the use of high-yield production strains. In this chapter we focus on genetic approaches for the generation of improved microalgal strains with enhanced lipid production. We discuss challenges and bottlenecks in the current genetic toolbox as well as strategies for genetic engineering of lipid biosynthetic pathways. Moreover, we describe the main findings and limitations of the experimental work performed in this thesis and discuss future perspectives for the generation of improved strains with enhanced lipid metabolism. 


\section{Microalgal lipids}

Oleaginous microalgae have attracted great commercial interest as sustainable source of oil for biodiesel purposes and in food industry as edible plant oil substitutes (Chisti, 2007; Fu et al., 2019; Ghiffary et al., 2019; Wijffels \& Barbosa, 2010). A vast number of microalgal lipids can be found in nature. Major lipid classes have been identified as storage compounds in the form of triacylglycerols (TAGs) and membrane lipids such as glycosylglycerides, phosphoglycerides and betaine ether lipids (Breuer et al., 2012; Guschina \& Harwood, 2006; Harwood \& Jones, 1989; Khan et al., 2018).

The most abundant lipids found in intracellular storage compounds are monoacylglycerols, diacylglycerols and triacylglycerols harbouring either saturated or monounsaturated fatty acyl groups. These lipids are of great interest for industrial purposes as source for biofuel production and their synthesis is commonly induced by nutrient limitation such as nitrogen starvation (Brennan \& Owende, 2010; Janssen et al., 2018). On the other hand, several microalgal species produce other commercially relevant fatty acids such as very long chain polyunsaturated fatty acids (VLC-PUFAs). For instance, eicosapentaenoic (EPA), docosahexaenoic (DHA) and arachidonic acid (ARA) can provide health benefits when used as dietary supplementation (Ghiffary et al., 2019; Guschina \& Harwood, 2006; Harwood \& Jones, 1989).

Commercial scale production of microalgal lipids is however not yet economically feasible due to high production costs (Ruiz et al., 2016). Biorefinery processes and photo-bioreactors require improvement, optimization and redesign. Moreover, extensive screening and selection of microalgal strains suitable for industrial use is required. The ideal microalgal strain should exhibit a high growth rate, high photosynthetic efficiency, high lipid productivity and should be robust and able to excrete the product of interest (Fabris et al., 2020; Fu et al., 2019; Hochman \& Zilberman, 2014; Ruiz et al., 2016; Sheehan et al., 1998). 
The optimization process and selection of strains that meet all required characteristics can be time-consuming and labour-intensive. Therefore, strain improvement via genetic engineering is proposed as a tool to increase nutrient and carbon capture, improve growth rates, reduce by-product formation and enhance the metabolic flux towards lipid production.

\section{Genetic engineering of microalgae}

In the past decades, strain improvement has been mainly achieved by Mutagens such as UV light, gamma and X-rays as well as chemicals mutagens such as $\mathrm{N}^{\prime}$-nitro-N-nitrosoguanidine (NTG) and ethyl methanesulfonate (EMS) have been applied for the creation of random mutations. A targeted approach has been the focus of current research in order to achieve major changes and more desirable results. Advances in sequencing and bioinformatic technologies along with the development of efficient DNA delivery systems have allowed the generation of genetically improved microalgal strains. Specific insertions, deletions or substitutions into the genomic DNA have been possible due to the identification of regulatory elements such as promoters, splicing signals, terminators, selection and reporter markers. In addition, other tools have been developed such as of mono/polycistronic expression systems, inducible or constitutive expression of endogenous/heterologous genes, chloroplastic/nuclear gene integration and genome-editing techniques (Doron et al., 2016; Park et al., 2019).

Although the first successful nuclear transformation of microalgae was achieved in Chlamydomonas reinhardtii about thirty years ago in 1990 (K. L. Kindle, 1990), up to date DNA delivery systems are still limited to a few microalgal species and thus considered to be a major bottleneck (Chapter 5). Variation in cell sizes, cell wall structures and composition among microalgal species do not allow standardization of such transformation methods. They require adaptation and optimization to avoid poor transformation efficiencies and reduce cell death after the treatment is applied. Moreover, successful transformation cannot be reached only by determining optimal delivery settings but also requires the use of functional expression vectors for the targeted 
strains. Consequently, a method to verify that extracellular molecules are effectively delivered into microalgal cells is essential to asses functionality of expression vectors, transformability and to improve transformation efficiencies.

In Chapter $\mathbf{2}$ we proposed a screening protocol that can be used to optimize electroporation-based transformation methods. This protocol can be used to assess delivery of exogenous molecules in poorly transformable strains or less well-studied microalgal species, as well as improve performance and increase insight in established protocols. We gave guidelines to find optimal electroporation settings by measuring cell permeability and viability using two fluorescent dyes: Sytox Green and propidium iodide, respectively. As expected, we demonstrated that higher voltages lead to higher cell permeabilization and reduced cell viability. We proposed to determine the optimal voltage for gene transfer for each species as the intersection between permeability and viability curves. The values obtained corresponded to the values described in literature thereby validating our method. In order to increase the reproducibility and applicability of the technique we assessed cell uptake of exogenous molecules using labelled DNA and proteins in four industrially relevant microalgal species with different physiological and cellular characteristics: Chlamydomonas reinhardtii, Chlorella vulgaris, Neochloris oleoabundans and Acutodesmus obliquus. Moreover, we achieved high efficiency during microalgal transformation when using the optimized protocol obtained from our screening method.

Furthermore, in Chapter $\mathbf{3}$ we provided an alternative transformation method based on bacterial conjugation. Conjugation is a natural mechanism commonly used as a transformation method for the delivery of DNA vectors from bacteria to other prokaryotic and eukaryotic cells (Lacroix \& Citovsky, 2016). It requires a conjugative bacterium carrying a plasmid that contains an origin of replication to allow replication of the plasmid and an origin of transfer and mob genes for DNA mobilization between the strains (Karas et al., 2015). In this chapter, we reported for the first time the successful transformation of the green microalgae Acutodesmus obliquus and Neochloris oleoabundans via bacterial conjugation. We adapted and optimized the conjugation conditions from previous reports 
(Diner et al., 2016; Karas et al., 2015) and designed and tested two plasmids for genome integration and episomal transformation in both strains. Our results showed higher transformation efficiencies for one of the targeted strains. We hypothesized that interaction between microalgae and bacteria might differ for both strains considering variations in cell size, cell wall structure and composition (Allard et al., 1998; D. Wang et al., 2015). On the other hand, we demonstrated that longer exposure times of microalgae to bacterial cells and higher initial bacterial concentration can influence transformation efficiencies.

Karas et al. (2015) (Karas et al., 2015) and Diner et al. (2016) (Diner et al., 2016) reported the successful transformation of diatom microalgae by transferring episomal plasmids containing a yeast-derived centromeric sequence. Episomal plasmids are self-replicating and non-integrating systems. They are commonly used to avoid random integration events into the strains' genome which can lead to undesirable perturbations in the microalgal metabolism. In our study, we selected positive transformants by identifying the presence of antibiotic resistance markers and expression of fluorescent reporting genes. However, episomes could not be rescued from positive transformants. Since we observed different fluorescence intensities in different mutants, we suggested that multiple integrations or integration positional effects in the genome might cause different expression levels (Doron et al., 2016). The centromere sequence used in our study has been adapted for diatom microalgal strains and thus a more appropriate centromeric sequence has to be identified to allow episomal maintenance and replication (Karas et al., 2015).

The method presented in this chapter offers advantages over traditional methods for its simplicity, no need of expensive equipment, reduction of cell death caused not only by electrical shocks but also physical damage induced by other transformation methods such as ballistics and agitation with glass beads (S. Kang et al., 2015; Muñoz et al., 2018). As observed in Chapter 2, transformation methods such as electroporation might induce the formation of micro-pores in the cell wall reducing the passage of bigger molecules and thus decreasing transformation efficiencies (Muñoz et al., 2018). Therefore, a 
conjugation-based method was proposed as an alternative for the delivery of relatively long DNA fragments allowing the expression of large set of genes in microalgal strains.

\section{Genetic engineering for enhanced lipid metabolism}

Since the first genetic transformation of Chlamydomonas reinhardtii in 1990 (K. L. Kindle, 1990), significant advances in all fields of genetics and genetic technology have allowed the genetic modification of microalgae. Although, the first use of such genetic tools for increasing lipid content in microalgae occurred in 1996 (Terri G. Dunahay et al., 1996), the lack of a genetic toolbox, genome availability, fast and reliable sequencing technology and understanding of lipid metabolism did not allowed further investigation up until 2011 (Chapter 5, figure 1a).

As presented in Chapter 4, the first demonstration of successful transformation of Phaeodactylum tricornutum occurred in 1996 (Apt et al., 1996), the first application of a genome-editing tool in microalgae by using RNA interference was achieved in Chlamydomonas in 1999 (Schroda et al., 1999) and the fully sequenced genomes of Chamydomonas, Phaeodactylum and Nannochloropsis were not available up until 2007, 2008 and 2012, respectively (Bowler et al., 2008; Merchant et al., 2007; Radakovits et al., 2012).

In Chapter 5, we provided a complete literature review about developments in genetics for the generation of microalgae strains with enhanced lipid content. We reviewed genetic techniques and strategies used throughout the last 30 years. Up to date $70 \%$ of the research aiming to increase lipid content via targeted genetic approaches has been done mainly in Chlamydomonas, Phaeodactylum and Nannochloropsis strains. Overall, many efforts have demonstrated that lipid content can be increased to a certain extent by targeting enzymes involved in the fatty acid synthesis pathway (FAS), Kennedy pathway, PUFAs and TAG metabolism, NADPH generation and by targeting transcriptional regulators among others. As presented in Chapter 5, 
interventions in the central carbon metabolism and cofactor supply (e.g. $\mathrm{NAD}(\mathrm{P}) \mathrm{H}$ ) has shown substantial lipid increase in most of the studies.

Improving the primary storage lipid triacylglycerol (TAG) has been the main focus of research due to great interest in industry for their potential use as source for biofuel production (Brennan \& Owende, 2010). It has been proposed that TAG biosynthesis takes place either in the chloroplast, in the endoplasmic reticulum (ER) using diacylglycerol (DAG) exported from the chloroplast or by producing both DAG and TAG directly in the ER (Li-Beisson et al., 2019). TAG biosynthesis occurs mainly in the Kennedy pathway, where a glycerol backbone is acylated via the action of three acyltransferases: glycerol-3-phosphate acyltransferase (GPAT), lysophosphatidic acid acyltransferase (LPAAT) and diacylglycerol acyltransferase (DGAT). Several studies have shown that increased expression of the genes encoding these enzymes can lead to increased lipid content. For instance, overexpression of GPAT in Phaeodactylum tricornutum showed 2-fold increase in TAG (Y. F. Niu et al., 2016), overexpression of LPAT in Chlamydomonas reinhardtii has increased the oil content by up to 1.2-fold (Yamaoka et al., 2016) and overexpression of DGAT in P. tricornutum led to 2.3-fold increase in TAGs (Zulu et al., 2017). Considering the importance of the Kennedy pathway in TAGs biosynthesis, it has become the most studied and genetically manipulated pathway in microalgal strains, resulting in more than 30 reports up to date (Chapter $\mathbf{5}$, figure 1b).

In Chapter 3, we successfully expressed single and multiple genes encoding for LPAT, GPAT and DGAT from Acutodesmus obliquus in N. oleoabundans. In order to enhance lipid production in $N$. oleoabundans, we performed heterologous expression of single and combined gene(s) and investigated their effect on growth, lipid composition and cellular biochemical composition of all transformant lines. We demonstrated that single gene overexpression resulted in 1.2- and 1.4-fold increase in total fatty acids and TAG while multi-gene overexpression resulted in 1.2-fold increase. Furthermore, we showed that single gene expression did not affect growth and photosynthetic efficiencies, and increase lipid productivities during nitrogen starvation conditions. On the 
other hand, multi-gene expression had a negative impact on growth rates, photosynthetic activity, carbohydrate and protein content. Enhancing the Kennedy pathway capacity via genetic engineering can help to further elucidate the lipid metabolism and lead to the generation of microalgal strains with higher economical potential for the successful production of lipids.

Overall, overexpression or knock-out approaches used to manipulate lipid metabolism have increased our understanding about the functionality of the several enzyme isoforms present in the different compartments and organelles. However, these strategies are hampered by the limited genetic knowledge, the lack of gene annotations and prediction tools for protein localization due to the great diversity of microalgal species and genera. In order to enhance the carbon flux towards lipid production in microalgae, a full rewiring of the central and lipid metabolism, a more thorough physiological understanding of microalgal metabolism, function and localization of the encoding enzymes are essential.

\section{Current challenges and bottlenecks}

Genetic engineering of microalgae still faces several challenges due to the limited molecular information available and lack of efficient genetic toolbox. Limited number of omics data availability and genetic tools have allowed the genetic engineering of only a few microalgal species. Nearly 16 out of approximately 600 described genera of microalgae have been engineered (Norton et al., 1996) (Chapter 4, figure1c) to enhance lipid content, of which around $80 \%$ of the publications correspond to the genus Chlamydomonas, Phaeodactylum, Nannochloropsis and Chlorella. Therefore, the main challenge is to make the genetic tools available for industrially relevant strains and increase omics availability for a large number of microalgal species. Up to date there is a limited number of fully annotated genomes, which does not allow effective and strategic design of genetic engineering approaches of targeted strains. Moreover, full gene annotation increases the accuracy and reliability of genome-scale metabolic models which can be constructed by using bioinformatic tools. Such tools can have a great impact in the design of metabolic engineering strategies as they can predict the overall effect of 
insertions, deletions or silencing of genes involved in specific metabolic pathways (Lauritano et al., 2019; W. R. Lin et al., 2019; Y. T. Zhang et al., 2019).

\section{Transformation methods}

The most common techniques for the delivery of exogenous DNA into microalgal cells are electroporation, Agrobacterium tumefaciens-mediated transformation (ATMT), ballistic systems and agitation with glass beads ( $\mathrm{K}$. Jeon et al., 2013; Karen L. Kindle, 1998; S. V. Kumar et al., 2004; C. Tan et al., 2005). Most of these techniques have been successfully applied in model strains such as Chlamydomonas reinhardtii, Phaeodactylum tricornutum, and Chlorella sp., however there is a lack of efficient techniques that can be applied to a broader range of microalgal strains.

The main cause for unsuccessful transformation of microalgae is due to the great variation in microalgal cell composition. Shape and sizes of the cells, cell wall structures and composition can vary significantly between microalgal species which obstruct the passage of extracellular genetic material into intracellular compartments (Allard et al., 1998; Azencott et al., 2007; Gimpel et al., 2015; Ortiz-Matamoros et al., 2018). For instance, the cell wall of $C$. reinhardtii lacks cellulose and is composed of hydroxyproline-rich glycoproteins (Macfie et al., 1994; Voigt, 1988) and the cell wall of $A$. obliquus consists of an inner cellulosic layer surrounded by a trilaminar structure known as algaenan containing glycoproteins, glucosamine and lipids (Allard et al., 1998; J. Burczyk et al., 1970; Jan Burczyk et al., 1999). The cell wall of C. vulgaris contains glucosamine, cellulose and other polysaccharides including rhamnose and arabinose (Pieper et al., 2012) while the cell wall of $N$. oleoabundans mainly consists of cellulose (Chantanachat, 1962; D. Wang et al., 2015). This makes some microalgal species more susceptible to permeabilization and more prone to uptake extracellular genetic material. Furthermore, variations in DNA fragment lengths or macromolecule sizes can influence transformation efficiencies (Muñoz et al., 2018), as well as the cells' ability to survive after a chemical or mechanical treatment is applied. Therefore, particular methods are 
needed for specific strains and thus a broader range of genetic tools have to be developed.

\section{Sequence optimization}

Currently, due to the lack of sequence annotation and understanding of their functionality, it is customary to use heterologous regulatory elements such as promoters and terminators in the design of expression systems. However, endogenous regulatory sequences could significantly increase gene expression (Vila et al., 2012). Moreover, inclusion of splicing signals for RNA processing are essential for the correct translation into functional proteins. The use of regulatory elements such as the enhancer HSP70A, RBCS2 introns and inclusion of splicing signals has been demonstrated to lead to higher expression levels (Doron et al., 2016). Moreover, exogenous genes may contain codons that are rarely used in the targeted strain. The absence of tRNAs that are compatible with the codon usage of the gene of interest can dramatically affect the expression, structure and function of the encoded proteins in the production host (Heitzer et al., 2007). Differences in codon usage have been reported in microalgal species, as well as for organelles such as chloroplast and nuclear genomes of the same species (Jarvis et al., 1992; León-Bañares et al., 2004). Therefore, codon optimization or harmonization of exogenous genes might be necessary for correct gene expression and protein folding.

\section{Nuclear/Chloroplast transformation systems}

Most of the successful achievements in microalgal biotechnology have reported nuclear expression systems as the main transformation approach. This strategy offers several advantages, including redirecting the expressed proteins into specific organelles as well as secretion (Doron et al., 2016). Another promising approach is chloroplastic transformation since transgenes can be integrated via homologous recombination at a desired location in the genome, contrarily to the nuclear transformation approach which usually results in random integration events. Chloroplastic transformation has been successfully achieved in the model strain $C$. reinhardtii using biolistic delivery and agitation of cell 
wall-deficient cells with glass beads coated with DNA (Boynton et al., 1988; Karen L. Kindle et al., 1991). However, there are still less methods for chloroplast transformation compared to those targeting the microalgal nucleus. The challenge remains in the identification of selection markers and regulatory elements such as inducible and constitutive promoters and UTRs that are functional for this organelle (Doron et al., 2016).

\section{Episomal expression}

The use of episomal vectors is an alternative to the commonly used vector systems. It provides advantages such as expression of a large set of genes thereby avoiding undesirable effects as it is not random integrated into the microalgae genome. The episome is maintained as a circular plasmid and functions as an extra chromosome in the cells. Karas et al. (2015), Diner et al. (2016) and Slattery et al. (2018) have demonstrated the successful transformation of episomes in two diatomic species, $P$. tricornutum and $T$. pseudonana as well as in the oleoaginous microalga Nannochloropsis oceanica. The vector has been adapted to contain a yeast-derived sequence that promoted its replication even in antibiotic-free medium (Diner et al., 2016; Poliner et al., 2018; Slattery et al., 2018).

As reported by Diner et al. (2016)(Diner et al., 2016), several combinations of the elements present in the centromere sequence and variations in GC content can lead to variations in transformation efficiencies. Therefore, successful use of this method requires an appropriate or optimized centromeric sequence to allow episomal maintenance and replication in other strains.

\section{Genome-editing techniques in microalgae}

Up to date, several genome-editing tools have been used in microalgae, such as zinc-finger nucleases (ZFNs), meganucleases (MNs), transcription activatorlike effector nucleases (TALEN), and clustered regularly interspaced short palindromic repeats (CRISPR/Cas9-Cpf1). All these tools introduce a doublestrand break in the host genome which is further repaired via non-homologous end-joining or homologous recombination. Moreover, they allow multiple gene 
edits simultaneously such as gene knock-out, knock-down and knock-in in the targeted genome (Jiang et al., 2014; W. R. Lin et al., 2019; Park et al., 2019; Y. T. Zhang et al., 2019). Most of these genome-editing tools have only been applied recently in microalgae and therefore require refinement to increase their efficiency across microalgal species.

The use of Cas9/single guide RNA ribonucleoprotein (RNP) has shown promising results (Baek et al., 2016; Shin et al., 2016). This approach allows the delivery of pre-assembled RNPs into the cells without relying in the integration of foreign DNA into the genome. Such an approach reduces off-target and toxic effects and improve efficiencies (G. Kumar et al., 2020). Genome-editing not only provides a powerful tool for precise and targeted genetic modifications but it also allows the generation of marker-free and non-transgenic strains thereby facilitating their use in industrial processes.

\section{Future prospects}

Although great progress has been achieved in the last years, the use of genetic engineering for the modification of microalgal strains is limited by the genetic toolbox available. Current research relies mainly on the use of constitutive promoters which often has a negative impact on growth. Consequently, development of highly and tightly regulated expression systems by using inducible promoters is desirable. This approach is ideal for inducing lipid production during nutrient deprived or limited conditions and reducing the negative impact during growth phase. At the moment nuclear transformation is the preferred approach for insertion of DNA sequences into the genome. Although a few studies have achieved targeted insertions into other organelles, there is still the need to develop chloroplast and mitochondria transformation methods for a broader variety of microalgal species. Furthermore, in order to design optimal metabolic engineering strategies, it is necessary to unravel metabolic networks and genetic regulation. There is great number of putative gene annotations that needs genetic and functional characterization. This can be investigated using bioinformatic tools, expression/transcription analysis and enzymatic assays. Genome-scale metabolic models are a great tool for gaining 
insight into microalgal metabolism and designing genetic engineering strategies. However, experimental characterization of enzymes, kinetic analysis and gene regulation is required to validate outcomes predicted with these models. The use of genome-editing tools can become useful to fine tune and validate such predictions by creating precise modification in specific metabolic pathways.

Since microalgae use $\mathrm{CO}_{2}$ and light for growth and product formation, future research needs to expand the current knowledge on carbon sequestration, rewiring and redirection of carbon fluxes, as well as maximizing light capture efficiencies. The generation of stable and marker-free genetically modified microalgae strains will become essential for industrial purposes and sustainable production of biomass and valuable compounds.

\section{Conclusion}

Microalgae are considered as promising biological platform for the production of valuable compounds and current research aims to bring such products to the market. In order to achieve industrial scale production, optimization of cultivation systems and improvement of microalgal strains are needed. In the past decade, advances in sequencing and omics technology have increased our understanding of lipid biosynthesis pathways. Genetic tool development and its use for the modification of microalgae has become a promising approach for the generation of improved strains with enhanced lipid productivities. Nevertheless, major bottlenecks need to be addressed in order to generate strains that meet the standards of a competitive industrial strain. Transformation methods that can be used in a broader variety of microalgal strains and their ability to target specific organelles such as chloroplast or nucleus is key for future success. In addition, sequence optimization that leads to correct transcription/translation is needed and the use of expression systems that do not rely on random integration (e.g. episomal expression systems). Currently, the use of genome-editing tools becomes essential for the modification of microalgal genome due to their accuracy, efficiency and their 
potential use for the generation of marker-free strains which can be used in industrial processes. 

Thesis summary 


\section{Summary}

The global demand for food, feed and fossil-based chemicals is rapidly increasing due to the continuously growing world population. Excessive and unsustainable consumption is causing anthropogenic effects resulting in high carbon emission, environmental pollution and depletion of natural resources. Currently, the main renewable feedstocks used as alternative to petrochemical processes are derived from agricultural crops. Higher plants are a great source of oils which can be used in food, feed, paint, lighting and fuel industries. However, cultivation of crops requires availability of vast areas of arable land, fresh water and minerals. On the other hand, depletion of petrochemical resources increases the demand for plant-based fuels, creating a direct competition with food and feed production. Therefore, novel renewable and sustainable alternatives are necessary to reduce the current environmental impact of traditional industrial processes.

Microalgae are considered as a promising sustainable alternative for the production of valuable commodities such as pigments, proteins, carbohydrates and lipids. They are capable of converting sunlight, water, $\mathrm{CO}_{2}$ and nutrients into biomass and products which attract interest from pharmaceutical, cosmetic, biofuel, food and feed industries. Furthermore, microalgae can reach significantly higher biomass concentrations and product yields per hectare compared to higher plants, they do not require vast available croplands and some species can be grown on wastewater or seawater, reducing the use of valuable natural resources.

Several microalgal species can accumulate large amounts of fatty acids when cultivated under stress conditions. At a cellular level, lipids such as triacylglycerols (TAGs) and polyunsaturated fatty acids (PUFAs) can serve either as structural components or storage compounds. On the other hand, they are a great source of valuable oils that can be used directly as nutritional products or as raw material for biofuel production by their conversion through a transesterification process. 
Although microalgae have the potential to become microbial cell factories for lipid production some limitations and challenges remain. In order to achieve industrial scale production and an economically feasible process, improvement, optimization and redesign of cultivation systems are required, as well as improvement of microalgae strains. Omics and genetic tool development are increasing our understanding of lipid metabolism. Such tools can accelerate the generation of strains with enhanced growth rates, photosynthetic efficiencies and lipid productivities. Therefore, genetic engineering has become a promising approach for the generation of improved strains with enhanced product formation, facilitating the reduction of the overall production costs.

The lack of genetic engineering tools for efficient delivery of genetic material into the cells is one of the main bottlenecks for successful genetic modification of microalgae. Several genetic methods have been developed over the years such as particle bombardment, electroporation, Agrobacterium tumefaciens mediated transformation (ATMT), and agitation with glass beads and silicon carbide whiskers. However, these techniques have been proven to work in a limited number of strains. Therefore, the development of a reliable, efficient and robust genetic toolbox that can be applied to poorly transformable strains, less well-studied or industrially relevant microalgal species is needed.

Poor intracellular delivery of DNA/protein molecules into microalgae cells is caused by the structure and composition of the cell wall and cell membrane of each species. In addition, successful delivery of these molecules into the cells cannot be assessed by determining transformability as their functionality is not always known in the studied microorganisms. Therefore, in Chapter $\mathbf{2}$ we proposed a screening tool for the optimization of electroporation-based transformation methods. We determined cell permeability and viability using Sytox Green and propidium iodide, respectively. In order to create a genetic toolbox that can be applied to a wide range of microalgal species, we tested our screening method in four industrially relevant species with different physiological and cellular characteristics: Chlamydomonas reinhardtii, Chlorella vulgaris, Neochloris oleoabundans and Acutodesmus obliquus. We successfully delivered labelled DNA and proteins into the cells and demonstrated that high 
transformation efficiencies can be accomplished when using functional plasmids and applying the predicted electroporation settings. Additionally, we increased transformation efficiencies by testing cell concentrations, light intensities and fragment sizes. This quick and effective method offers guidance to determine suitable transformation conditions for non-transformed microalgae species and increases insight on established transformation protocols.

Transformation efficiency is species-dependent and some methods present several limitations: DNA fragment lengths, macromolecule size or high cell death after the treatment is applied can impede or reduce transformation efficiencies. Therefore, a variety of transformation methods are required to engineer a wide range of microalgae species. In Chapter 3, we presented a conjugation-based transformation technique. We successfully engineered two green oleaginous microalgae strains Acutodesmus obliquus and Neochloris oleoabundans due to their great potential as production platforms for triacylglycerol. Successful transformation of both strains was achieved for the first time by transferring an expression vector via $E$. coli conjugation. We further attempted to deliver episomal plasmids into the cells. Episomes can be maintained as circular plasmid and function as extra chromosomes which could avoid random integration events. In our study, we did not accomplish successful rescue of the episomes from positive transformants and we hypothesized that random integration might have occurred. This technique was proposed as an alternative transformation approach over traditional methods when successful transformation is impeded due to high cell death or physical damage and limited passage of bigger molecules through the cell wall. Delivery of relatively long DNA fragments containing large set of genes could increase TAG levels further or modify the fatty acid composition in microalgae.

Since lipid production by microalgae is not yet economically feasible, in Chapter 4 we made use of the previously developed tools for the enhancement of TAG production in $N$. oleoabundans. We overexpressed genes encoding enzymes involved in the Kennedy pathway which are the main responsible for TAG production in microalgae. Single and multi-gene expression approaches were applied by expressing genes encoding for glycerol-3-phosphate 
acyltransferase (GPAT), lysophosphatidic acid acyltransferase (LPAT) and diacylglycerol acyltransferase (DGAT) from $A$. obliquus into $N$. oleoabundans. We achieved the successful transformation of $N$. oleoabundans and strains overexpressing single genes were able to produced up to $52 \%$ and $45 \% \mathrm{~g}$. $\mathrm{gDW}^{-1}$ in total fatty acids (TFAs) and TAGs, which corresponds to 1.3- and 1.4fold increase with respect to the wild type strain. On the other hand, simultaneous expression of genes LPAT, GPAT and DGAT in a single expression system resulted in $49 \%$ and $39 \% \mathrm{~g} \cdot \mathrm{gDW}^{-1}$ increase in TFAs and TAGs, which corresponds to 1.2-fold increase compared to the wild type. Single gene expression resulted in higher lipid titers and productivities without having a significant impact on growth rates and photosynthetic activity. Conversely, simultaneous expression of LPAT, GPAT and DGAT genes resulted in $52 \%$ lower growth rate, $14 \%$ lower photosynthetic activity and significant changes in cell composition (i.e., decreased carbohydrates and protein contents and increased pigments content during nitrogen starved condition). Overall, our results showed that heterologous gene expression can significantly enhanced lipid accumulation in $N$. oleoabundans and provides a valid approach for the generation of microalgal strains with higher economical potential for the production of lipids.

In Chapter $\mathbf{5}$ we provided a complete overview of developments in genetics for the generation of microalgae strains with enhanced lipid content. We reviewed important advances in omics technology and genetic tool development as well as its application in microalgae aiming to improve carbon fluxes towards fatty acid synthesis. Such developments have increased our understanding in lipid metabolism allowing the design of experimental strategies that can alter fatty acid chain length, branching and degree of unsaturation. In this chapter we provided an overview of genetic strategies applied up to date in microalgae, including modification of fatty acid synthesis pathway (FAS), Kennedy pathway, PUFA and TAG metabolism, and modification of enzymes involved in NADPH generation and gene(s) regulation.

In Chapter 6 we discussed advances in the microalgal biotechnology field including most studied strains, genetic tool development, as well as techniques 
for DNA delivery into microalgae cells and strain improvement approach described in this thesis. We described major bottlenecks and challenges that need to be addressed in order to generate strains that can meet the standards of a competitive industrial strain. Development of genetic tools that can target organelles such as chloroplast or nucleus, sequence optimization for correct transcription and translation, as well of avoidance of random integration of expression systems are some of the issues that need to be tackled. Furthermore, we proposed that development of genome-editing tools is key for accurate and efficient modification of microalgae which will allow the generation of marker-free strains that can be used in industrial processes. 



\section{References}


Abbriano, R., Vardar, N., Yee, D., \& Hildebrand, M. (2018). Manipulation of a glycolytic regulator alters growth and carbon partitioning in the marine diatom Thalassiosira pseudonana. Algal Research, 32, 250-258.

Agarwal, P., Baranwal, V. K., \& Khurana, P. (2019). Genome-wide Analysis of bZIP Transcription Factors in wheat and Functional Characterization of a TabZIP under Abiotic Stress. Scientific Reports, 9(1), 4608.

Ahmad, I., Sharma, A. K., Daniell, H., \& Kumar, S. (2015). Altered lipid composition and enhanced lipid production in green microalga by introduction of brassica diacylglycerol acyltransferase 2 . Plant Biotechnology Journal, 13(4), 540-550.

Ajjawi, I., Verruto, J., Aqui, M., Soriaga, L. B., Coppersmith, J., Kwok, K., ... Moellering, E. R. (2017). Lipid production in Nannochloropsis gaditana is doubled by decreasing expression of a single transcriptional regulator. Nature Biotechnology, 35(7), 647-652.

Allard, B., Templier, J., \& Largeau, C. (1998). An improved method for the isolation of artifact-free algaenans from microalgae. Organic Geochemistry, 28(9-10), 543-548.

Alptekin, E., Canakci, M., \& Sanli, H. (2014). Biodiesel production from vegetable oil and waste animal fats in a pilot plant. Waste Management, 34(11), 2146-2154.

Apt, K. E., Grossman, A. R., \& Kroth-Pancic, P. G. (1996). Stable nuclear transformation of the diatom Phaeodactylum tricornutum. Molecular and General Genetics MGG, 252(5), 572-579.

Aratboni, H. A., Rafiei, N., Garcia-Granados, R., Alemzadeh, A., \& MoronesRamírez, J. R. (2019). Biomass and lipid induction strategies in microalgae for biofuel production and other applications. Microbial Cell Factories, 18, 178.

Azencott, H. R., Peter, G. F., \& Prausnitz, M. R. (2007). Influence of the cell wall on intracellular delivery to algal cells by electroporation and sonication. Ultrasound in Medicine and Biology, 33(11), 1805-1817.

Baek, K., Kim, D. H., Jeong, J., Sim, S. J., Melis, A., Kim, J. S., Jin, E., \& Bae, S. (2016). DNA-free two-gene knockout in Chlamydomonas reinhardtii via CRISPR-Cas9 ribonucleoproteins. Scientific Reports, 6(1), 1-7.

Bajhaiya, A. K., Dean, A. P., Zeef, L. A. H., Webster, R. E., \& Pittman, J. K. (2016). PSR1 is a global transcriptional regulator of phosphorus deficiency responses and carbon storage metabolism in Chlamydomonas reinhardtii. Plant Physiology, 170(3), 1216-34.

Balamurugan, S., Wang, X., Wang, H. L., An, C. J., Li, H., Li, D. W., Yang, W. 
D., Liu, J. S., \& Li, H. Y. (2017). Occurrence of plastidial triacylglycerol synthesis and the potential regulatory role of AGPAT in the model diatom Phaeodactylum tricornutum. Biotechnology for Biofuels, 10(1):97.

Banerjee, C., Dubey, K. K., \& Shukla, P. (2016). Metabolic engineering of microalgal based biofuel production: Prospects and challenges. Frontiers in Microbiology, 7:432.

Barbosa, M. J., Albrecht, M., \& Wijffels, R. H. (2003). Hydrodynamic stress and lethal events in sparged microalgae cultures. Biotechnology and Bioengineering, 83(1), 112-120.

Barkia, I., Saari, N., \& Manning, S. R. (2019). Microalgae for high-value products towards human health and nutrition. Marine Drugs, 17(5), 304.

Barten, R. J. P., Wijffels, R. H., \& Barbosa, M. J. (2020). Bioprospecting and characterization of temperature tolerant microalgae from Bonaire. Algal Research, 50, 102008.

Beacham, T. A., Macia, V. M., Rooks, P., White, D. A., \& Ali, S. T. (2015). Altered lipid accumulation in Nannochloropsis salina CCAP849/3 following EMS and UV induced mutagenesis. Biotechnology Reports, 7, 87-94.

Beacham, Tracey A., \& Ali, S. T. (2016). Growth dependent silencing and resetting of DGA1 transgene in Nannochloropsis salina. Algal Research, $14,65-71$.

Benvenuti, G., Lamers, P. P., Breuer, G., Bosma, R., Cerar, A., Wijffels, R. H., \& Barbosa, M. J. (2016). Microalgal TAG production strategies: Why batch beats repeated-batch. Biotechnology for Biofuels, 9(1), 64.

Benvenuti, G., Ruiz, J., Lamers, P. P., Bosma, R., Wijffels, R. H., \& Barbosa, M. J. (2017). Towards microalgal triglycerides in the commodity markets. Biotechnology for Biofuels, 10(1):188.

Blatti, J. L., Beld, J., Behnke, C. A., Mendez, M., Mayfield, S. P., \& Burkart, M. D. (2012). Manipulating fatty acid biosynthesis in microalgae for biofuel through protein-protein interactions. PLOS ONE, 7(9), e42949.

Boelen, P., van Dijk, R., Damsté, J. S. S., Rijpstra, W. I. C., \& Buma, A. G. J. (2013). On the potential application of polar and temperate marine microalgae for EPA and DHA production. AMB Express, 3(1), 1-9.

Bowler, C., Allen, A. E., Badger, J. H., Grimwood, J., Jabbari, K., Kuo, A., ... Grigoriev, I. V. (2008). The Phaeodactylum genome reveals the evolutionary history of diatom genomes. Nature, 456(7219), 239-244.

Boynton, J. E., Gillham, N. W., Harris, E. H., Hosler, J. P., Johnson, A. M., Jones, A. R., Randolph-Anderson, B. L., Robertson, D., Klein, T. M., Shark, K. B., 
\& Sanford, J. C. (1988). Chloroplast transformation in Chlamydomonas with high velocity microprojectiles. Science, 240(4858), 1534-1538.

Brennan, L., \& Owende, P. (2010). Biofuels from microalgae-A review of technologies for production, processing, and extractions of biofuels and co-products. Renewable and Sustainable Energy Reviews, 14: 557-77.

Breuer, G., Evers, W. A. C., de Vree, J. H., Kleinegris, D. M. M., Martens, D. E., Wijffels, R. H., \& Lamers, P. P. (2013). Analysis of fatty acid content and composition in microalgae. Journal of Visualized Experiments : JoVE, (80), e50628.

Breuer, G., Lamers, P. P., Martens, D. E., Draaisma, R. B., \& Wijffels, R. H. (2012). The impact of nitrogen starvation on the dynamics of triacylglycerol accumulation in nine microalgae strains. Bioresource Technology, 124, 217-226.

Burczyk, J., Grzybek, H., Banaś, J., \& Banaś, E. (1970). Presence of cellulase in the algae Scenedesmus. Experimental Cell Research, 63(2-3), 451453.

Burczyk, Jan, Śmietana, B., Termińska-Pabis, K., Zych, M., \& Kowalowski, P. (1999). Comparison of nitrogen content amino acid composition and glucosamine content of cell walls of various chlorococcalean algae. Phytochemistry, 51(4), 491-497.

Cagnon, C., Mirabella, B., Nguyen, H. M., Beyly-Adriano, A., Bouvet, S., Cuiné, S., Beisson, F., Peltier, G., \& Li-Beisson, Y. (2013). Development of a forward genetic screen to isolate oil mutants in the green microalga Chlamydomonas reinhardtii. Biotechnology for Biofuels, 6(1), 178.

Canãvate, J. P., Armada, I., Riós, J. L., \& Hachero-Cruzado, I. (2016). Exploring occurrence and molecular diversity of betaine lipids across taxonomy of marine microalgae. Phytochemistry, 124, 68-78.

Caporgno, M. P., \& Mathys, A. (2018). Trends in microalgae incorporation into innovative food products with potential health benefits. Frontiers in Nutrition, 5, 58.

Carreres, B. M., de Jaeger, L., Springer, J., Barbosa, M. J., Breuer, G., van den End, E. J., ... Martens, D. E. (2017). Draft genome sequence of the oleaginous green alga Tetradesmus obliquus UTEX 393. Genome Announcements, 5(3).

Chantanachat, S. (1962). Phycological studies II. Some algae from arid soils. University of Texas Publications, 6218, 1-74.

Chen, C.-Y., Kao, A.-L., Tsai, Z.-C., Chow, T.-J., Chang, H.-Y., Zhao, X.-Q., Chen, P.-T., Su, H.-Y., \& Chang, J.-S. (2016). Expression of type 2 
diacylglycerol acyltransferse gene DGTT1 from Chlamydomonas reinhardtii enhances lipid production in Scenedesmus obliquus. Biotechnology Journal, 11(3), 336-344.

Chen, J.-W., Liu, W.-J., Hu, D.-X., Wang, X., Balamurugan, S., Alimujiang, A., Yang, W.-D., Liu, J.-S., \& Li, H.-Y. (2017). Identification of a malonyl CoAacyl carrier protein transacylase and its regulatory role in fatty acid biosynthesis in oleaginous microalga Nannochloropsis oceanica. Biotechnology and Applied Biochemistry, 64(5), 620-626.

Chien, L. J., Hsu, T. P., Huang, C. C., Teng, K., \& Hsieh, H. J. (2015). Novel codon-optimization genes encoded in Chlorella for triacylglycerol accumulation. In Energy Procedia, 75, 44-55.

Chisti, Y. (2007). Biodiesel from microalgae. Biotechnology Advances, 25(3), 294-306.

Chou, H. H., Su, H. Y., Song, X. Di, Chow, T. J., Chen, C. Y., Chang, J. S., \& Lee, T. M. (2019). Isolation and characterization of Chlorella sp. mutants with enhanced thermo- And $\mathrm{CO}_{2}$ tolerances for $\mathrm{CO}_{2}$ sequestration and utilization of flue gases. Biotechnology for Biofuels, 12(1), 1-14.

Chow, K. C., \& Tung, W. L. (1999). Electrotransformation of Chlorella vulgaris. Plant Cell Reports, 18(9), 778-780.

Christaki, E., Florou-Paneri, P., \& Bonos, E. (2011). Microalgae: a novel ingredient in nutrition. International Journal of Food Sciences and Nutrition, 62(8), 794-799.

Chu, W.-L. (2017). Strategies to enhance production of microalgal biomass and lipids for biofuel feedstock. European Journal of Phycology, 52(4), 419437.

Chungjatupornchai, W., \& Fa-aroonsawat, S. (2020). Enhanced triacylglycerol production in oleaginous microalga Neochloris oleoabundans by cooverexpression of lipogenic genes: Plastidial LPAAT1 and ER-located DGAT2. Journal of Bioscience and Bioengineering, 131(2):124-130.

Chungjatupornchai, W., Kitraksa, P., \& Fa-aroonsawat, S. (2016). Stable nuclear transformation of the oleaginous microalga Neochloris oleoabundans by electroporation. Journal of Applied Phycology, 28(1), 191-199.

Cook, O., \& Hildebrand, M. (2016). Enhancing LC-PUFA production in Thalassiosira pseudonana by overexpressing the endogenous fatty acid elongase genes. Journal of Applied Phycology, 28(2), 897-905.

Cornish-Bowden, A. (2014). Current IUBMB recommendations on enzyme nomenclature and kinetics. Perspectives in Science, 1(1-6), 74-87. 
Corrêa, L. G. G., Riaño-Pachón, D. M., Schrago, C. G., dos Santos, R. V., Mueller-Roeber, B., \& Vincentz, M. (2008). The role of bZIP transcription factors in green plant evolution: adaptive features emerging from four founder genes. PloS One, 3(8), e2944.

Daboussi, F., Leduc, S., Maréchal, A., Dubois, G., Guyot, V., Perez-Michaut, C., Amato, A., Falciatore, A., Juillerat, A., Beurdeley, M., Voytas, D. F., Cavarec, L., \& Duchateau, P. (2014). Genome engineering empowers the diatom Phaeodactylum tricornutum for biotechnology. Nature Communications, 5(1), 1-7.

Dahlqvist, A., Stahl, U., Lenman, M., Banas, A., Lee, M., Sandager, L., Ronne, H., \& Stymne, S. (2000). Phospholipid:diacylglycerol acyltransferase: an enzyme that catalyzes the acyl-CoA-independent formation of triacylglycerol in yeast and plants. Proceedings of the National Academy of Sciences of the United States of America, 97(12), 6487-92.

Daniels, R. W., Rossano, A. J., Macleod, G. T., \& Ganetzky, B. (2014). Expression of multiple transgenes from a single construct using viral $2 \mathrm{~A}$ peptides in Drosophila. PLOS ONE, 9(6).

de Jaeger, L., Carreres, B. M., Springer, J., Schaap, P. J., Eggink, G., Martins Dos Santos, V. A. P., Wijffels, R. H., \& Martens, D. E. (2018). Neochloris oleoabundans is worth its salt: Transcriptomic analysis under salt and nitrogen stress. PLOS ONE, 13(4).

De Jaeger, L., Verbeek, R. E. M., Draaisma, R. B., Martens, D. E., Springer, J., Eggink, G., \& Wijffels, R. H. (2014). Superior triacylglycerol (TAG) accumulation in starchless mutants of Scenedesmus obliquus: (I) mutant generation and characterization. Biotechnology for Biofuels, 7(1), 69.

de Mooij, T., Janssen, M., Cerezo-Chinarro, O., Mussgnug, J. H., Kruse, O., Ballottari, M., Bassi, R., Bujaldon, S., Wollman, F. A., \& Wijffels, R. H. (2015). Antenna size reduction as a strategy to increase biomass productivity: A great potential not yet realized. Journal of Applied Phycology, 27(3), 1063-1077.

Deng, X.-D., Gu, B., Li, Y.-J., Hu, X.-W., Guo, J.-C., \& Fei, X.-W. (2012). The roles of acyl-CoA: diacylglycerol acyltransferase 2 genes in the biosynthesis of triacylglycerols by the green algae Chlamydomonas reinhardtii. Molecular Plant, 5(4), 945-7.

Deng, X. D., Cai, J. J., \& Fei, X. W. (2013). Involvement of phosphatidate phosphatase in the biosynthesis of triacylglycerols in Chlamydomonas reinhardtii. Journal of Zhejiang University: Science B, 14(12), 1121-1131.

Dinamarca, J., Levitan, O., Kumaraswamy, G. K., Lun, D. S., \& Falkowski, P. G. (2017a). Overexpression of a diacylglycerol acyltransferase gene in Phaeodactylum tricornutum directs carbon towards lipid biosynthesis. 
Journal of Phycology, 53(2), 405-414.

Diner, R. E., Bielinski, V. A., Dupont, C. L., Allen, A. E., \& Weyman, P. D. (2016). Refinement of the diatom episome maintenance sequence and improvement of conjugation-based dna delivery methods. Frontiers in Bioengineering and Biotechnology, 4(8), 65.

Doron, L., Segal, N., \& Shapira, M. (2016). Transgene expression in microalgae-from tools to applications. Frontiers in Plant Science, 7, 505.

Draaisma, R. B., Wijffels, R. H., Slegers, P. M., Brentner, L. B., Roy, A., \& Barbosa, M. J. (2013). Food commodities from microalgae. Current Opinion in Biotechnology, 24(2), 169-177.

Drincovich, M. F., Casati, P., \& Andreo, C. S. (2001). NADP-malic enzyme from plants: a ubiquitous enzyme involved in different metabolic pathways. FEBS Letters, 490(1-2), 1-6.

Dubois, M., Gilles, K. A., Hamilton, J. K., Rebers, P. A., \& Smith, F. (1956). Colorimetric method for determination of sugars and related substances. Analytical Chemistry, 28, 350-356.

Dunahay, T. G. (1993). Transformation of Chlamydomonas reinhardtii with silicon carbide whiskers. BioTechniques, 15(3), 452-460.

Dunahay, Terri G., Jarvis, E. E., Dais, S. S., \& Roessler, P. G. (1996). Manipulation of microalgal lipid production using genetic engineering. Applied Biochemistry and Biotechnology - Part A Enzyme Engineering and Biotechnology, 57-58(1), 223-231.

Duong, V. T., Thomas-Hall, S. R., \& Schenk, P. M. (2015). Growth and lipid accumulation of microalgae from fluctuating brackish and sea water locations in South East Queensland-Australia. Frontiers in Plant Science, $6,359$.

Economou, C., Wannathong, T., Szaub, J., \& Purton, S. (2014). A simple, lowcost method for chloroplast transformation of the green alga Chlamydomonas reinhardtii. Methods in Molecular Biology, 1132, 401411.

Eichenberger, W. (1993). Betaine lipids in lower plant. Distribution of DGTS, DGTA and phospholipids, and the intracellular localization and site of biosynthesis of DGTS. Plant Cell Physiology, 33, 427-436.

Eugeni Piller, L., Besagni, C., Ksas, B., Rumeau, D., Bréhélin, C., Glauser, G., Kessler, F., \& Havaux, M. (2011). Chloroplast lipid droplet type II NAD(P)H quinone oxidoreductase is essential for prenylquinone metabolism and vitamin K1 accumulation. Proceedings of the National Academy of Sciences of the United States of America, 108(34), 14354-9. 
Fabris, M., Abbriano, R. M., Pernice, M., Sutherland, D. L., Commault, A. S., Hall, C. C., Labeeuw, L., McCauley, J. I., Kuzhiuparambil, U., Ray, P., Kahlke, T., \& Ralph, P. J. (2020). Emerging technologies in algal biotechnology: toward the establishment of a sustainable, algae-based bioeconomy. Frontiers in Plant Science, 11, 279.

Fan, Jianhua, Ning, K., Zeng, X., Luo, Y., Wang, D., Hu, J., ... Li, Y. (2015). Genomic foundation of starch-to-lipid switch in oleaginous Chlorella spp. Plant Physiology, 169(4), 2444-2461.

Fan, Jilian, Andre, C., \& Xu, C. (2011). A chloroplast pathway for the de novo biosynthesis of triacylglycerol in Chlamydomonas reinhardtii. FEBS Letters, 585(12), 1985-1991.

Fan, Y., Yuan, C., Jin, Y., Hu, G. R., \& Li, F. L. (2018). Characterization of 3ketoacyl-CoA synthase in a nervonic acid producing oleaginous microalgae Mychonastes afer. Algal Research, 31, 225-231.

Farhad Talebi, A., Tohidfar, M., Bagheri, A., Lyon, S. R., Salehi-Ashtiani, K., \& Tabatabaei, M. (2014). Manipulation of carbon flux into fatty acid biosynthesis pathway in Dunaliella salina using AccD and ME genes to enhance lipid content and to improve produced biodiesel quality. Biofuel Research Journal, 1(3), 91-97.

Faried, M., Samer, M., Abdelsalam, E., Yousef, R. S., Attia, Y. A., \& Ali, A. S. (2017). Biodiesel production from microalgae: Processes, technologies and recent advancements. Renewable and Sustainable Energy Reviews, 79, 893-913.

Fei, X., Li, X., Li, P., \& Deng, X. (2017). Involvement of Chlamydomonas DNA damage tolerence gene UBC2 in lipid accumulation. Algal Research, 22, 148-159.

Ferreira, G. F., Ríos Pinto, L. F., Carvalho, P. O., Coelho, M. B., Eberlin, M. N., Maciel Filho, R., \& Fregolente, L. V. (2019). Biomass and lipid characterization of microalgae genera Botryococcus, Chlorella, and Desmodesmus aiming high-value fatty acid production. Biomass Conversion and Biorefinery, 1-15.

Foo, S. C., Khong, N. M. H., \& Md. Yusoff, F. (2019). Manipulation of microalgal lipid production: A genetic engineering aspect. In Microalgae Biotechnology for Development of Biofuel and Wastewater Treatment (pp. 179-209). Springer Singapore.

Fu, W., Nelson, D. R., Mystikou, A., Daakour, S., \& Salehi-Ashtiani, K. (2019). Advances in microalgal research and engineering development. Current Opinion in Biotechnology, 59, 157-164.

Fukuda, S., Hirasawa, E., Takemura, T., Takahashi, S., Chokshi, K., Pancha, I., 
Tanaka, K., \& Imamura, S. (2018). Accelerated triacylglycerol production without growth inhibition by overexpression of a glycerol-3-phosphate acyltransferase in the unicellular red alga Cyanidioschyzon merolae. Scientific Reports, 8(1), 1-12.

Gao, H., Wright, D. A., Li, T., Wang, Y., Horken, K., Weeks, D. P., Yang, B., \& Spalding, M. H. (2014). TALE activation of endogenous genes in Chlamydomonas reinhardtii. Algal Research, 5(1), 52-60.

Gargouri, M., Bates, P. D., Park, J.-J., Kirchhoff, H., \& Gang, D. R. (2017). Functional photosystem I maintains proper energy balance during nitrogen depletion in Chlamydomonas reinhardtii, promoting triacylglycerol accumulation. Biotechnology for Biofuels, 10(1), 89.

Gerald E, E., \& Carlos S, A. (1992). NADP-malic enzyme from plants. Phytochemistry, 31(6), 1845-1857.

Gharabaghi, M., Delavai Amrei, H., Moosavi Zenooz, A., Shahrivar Guzullo, J., \& Zokaee Ashtiani, F. (2015). Biofuels: bioethanol, biodiesel, biogas, biohydrogen from plants and microalgae. In: Lichtfouse E., Schwarzbauer J., Robert D. (eds) CO2 Sequestration, Biofuels and Depollution. Environmental Chemistry for a Sustainable World (pp. 233-274). Springer Cham.

Ghiffary, M. R., Kim, H. U., \& Chang, Y. K. (2019). Metabolic engineering strategies for the enhanced microalgal production of long-chain polyunsaturated fatty acids (LC-PUFAs). Biotechnology Journal, 14(6), 1900043.

Gim, G. H., Kim, J. K., Kim, H. S., Kathiravan, M. N., Yang, H., Jeong, S. H., \& Kim, S. W. (2014). Comparison of biomass production and total lipid content of freshwater green microalgae cultivated under various culture conditions. Bioprocess and Biosystems Engineering, 37(2), 99-106.

Gimpel, J. A., Henríquez, V., \& Mayfield, S. P. (2015). In metabolic engineering of eukaryotic microalgae: Potential and challenges come with great diversity. Frontiers in Microbiology, 6, 1376.

Gimpel, J. A., Specht, E. A., Georgianna, D. R., \& Mayfield, S. P. (2013). Advances in microalgae engineering and synthetic biology applications for biofuel production. Current Opinion in Chemical Biology, 17(3), 489-495.

Gomma, A. E., Lee, S.-K., Sun, S. M., Yang, S. H., \& Chung, G. (2015). Improvement in oil production by increasing malonyl-coa and glycerol-3phosphate pools in Scenedesmus quadricauda. Indian Journal of Microbiology, 55(4), 447-455.

Gong, Y., Guo, X., Wan, X., Liang, Z., \& Jiang, M. (2011). Characterization of a novel thioesterase (PtTE) from Phaeodactylum tricornutum. Journal of 
Basic Microbiology, 51(6), 666-672.

Gorman, D. S., \& Levine, R. P. (1965). Cytochrome f and plastocyanin: their sequence in the photosynthetic electron transport chain of Chlamydomonas reinhardii. Proceedings of the National Academy of Sciences of the United States of America, 54(6), 1665-1669.

Griffiths, M. J., \& Harrison, S. T. L. (2009). Lipid productivity as a key characteristic for choosing algal species for biodiesel production. Journal of Applied Phycology, 21(5), 493-507.

Guo, S. L., Zhao, X. Q., Tang, Y., Wan, C., Alam, M. A., Ho, S. H., Bai, F. W., \& Chang, J. S. (2013). Establishment of an efficient genetic transformation system in Scenedesmus obliquus. Journal of Biotechnology, 163(1), 61-68.

Guschina, I. A., \& Harwood, J. L. (2006). Lipids and lipid metabolism in eukaryotic algae. Progress in Lipid Research, 45(2), 160-186.

Hamilton, M. L., Haslam, R. P., Napier, J. A., \& Sayanova, O. (2014). Metabolic engineering of Phaeodactylum tricornutum for the enhanced accumulation of omega-3 long chain polyunsaturated fatty acids. Metabolic Engineering, $22,3-9$.

Hamilton, M., Powers, S., Napier, J., \& Sayanova, O. (2016). Heterotrophic production of omega-3 long-chain polyunsaturated fatty acids by trophically converted marine diatom Phaeodactylum tricornutum. Marine Drugs, 14(3), 53.

Han, H.-S., Kang, G., Kim, J. S., Choi, B. H., \& Koo, S.-H. (2016). Regulation of glucose metabolism from a liver-centric perspective. Experimental \& Molecular Medicine, 48(3), e218-e218.

Hanaki, K., \& Portugal-Pereira, J. (2018). The effect of biofuel production on greenhouse gas emission reductions. In: Takeuchi K., Shiroyama $H_{\text {., Saito }}$ O., Matsuura M. (eds) Biofuels and Sustainability. Science for Sustainable Societies (pp. 53-71). Springer, Tokyo.

Hannon, M., Gimpel, J., Tran, M., Rasala, B., \& Mayfield, S. (2010). Biofuels from algae: Challenges and potential. Biofuels, 1(5), 763-784.

Harwood, J. L., \& Jones, A. L. (1989). Lipid metabolism in algae. Advances in Botanical Research, 16, 1-53.

Haslam, R. P., Hamilton, M. L., Economou, C. K., Smith, R., Hassall, K. L., Napier, J. A., \& Sayanova, O. (2020). Overexpression of an endogenous type 2 diacylglycerol acyltransferase in the marine diatom Phaeodactylum tricornutum enhances lipid production and omega-3 long-chain polyunsaturated fatty acid content. Biotechnology for Biofuels, 13(1), 87. 
Hauschild, R., \& von Schaewen, A. (2003). Differential regulation of glucose-6phosphate dehydrogenase isoenzyme activities in potato. Plant Physiology, 133(1), 47-62.

He, L., Xiao, Y., Gebreselassie, N., Zhang, F., Antoniewicz, M. R., Tang, Y. J., \& Peng, L. (2014). Central metabolic responses to the overproduction of fatty acids in Escherichia coli based on 13 C-metabolic flux analysis. Biotechnology and Bioengineering, 111(3), 575-585.

Hegel, P., Martín, L., Popovich, C., Damiani, C., Pancaldi, S., Pereda, S., \& Leonardi, P. (2017). Biodiesel production from Neochloris oleoabundans by supercritical technology. Chemical Engineering and Processing: Process Intensification, 121, 232-239.

Heitzer, M., Eckert, A., Fuhrmann, M., \& Griesbeck, C. (2007). Influence of codon bias on the expression of foreign genes in microalgae. Advances in Experimental Medicine and Biology, 616, 46-53.

Herbert, D., Phipps, P. J., \& Strange, R. E. (1971). Chemical analysis of microbial cells. Methods in Microbiology, 5, 209-344.

Hochman, G., \& Zilberman, D. (2014). Algae farming and its bio-products. In Plants and BioEnergy (pp. 49-64). Springer New York.

Hooykaas, P. J. J. (2004). Transformation mediated by Agrobacterium tumefaciens. In Advances in Fungal Biotechnology for Industry, Agriculture, and Medicine (pp. 41-65). Springer US.

Hopes, A., Nekrasov, V., Kamoun, S., \& Mock, T. (2016). Editing of the urease gene by CRISPR-Cas in the diatom Thalassiosira pseudonana. Plant Methods, 12(1), 49.

Hsieh, H. J., Su, C. H., \& Chien, L. J. (2012). Accumulation of lipid production in Chlorella minutissima by triacylglycerol biosynthesis-related genes cloned from Saccharomyces cerevisiae and Yarrowia lipolytica. Journal of Microbiology, 50(3), 526-534.

Hu, J., Wang, D., Li, J., Jing, G., Ning, K., \& Xu, J. (2014). Genome-wide identification of transcription factors and transcription-factor binding sites in oleaginous microalgae Nannochloropsis. Scientific Reports, 4, 5454.

Huang, A. H. C. (1992). Oil bodies and oleosins in seeds. Annual Review of Plant Physiology and Plant Molecular Biology, 43, 177-200.

Huang, L.-F., Lin, J.-Y., Pan, K.-Y., Huang, C.-K., \& Chu, Y.-K. (2015). Overexpressing ferredoxins in Chlamydomonas reinhardtii increase starch and oil yields and enhance electric power production in a photo microbial fuel cell. International Journal of Molecular Sciences, 16(8), 1930819325. 
Hwangbo, K., Ahn, J. W., Lim, J. M., Park, Y. Il, Liu, J. R., \& Jeong, W. J. (2014). Overexpression of stearoyl-ACP desaturase enhances accumulations of oleic acid in the green alga Chlamydomonas reinhardtii. Plant Biotechnology Reports, 8(2), 135-142.

Ibáñez-Salazar, A., Rosales-Mendoza, S., Rocha-Uribe, A., Ramírez-Alonso, J. I., Lara-Hernández, I., Hernández-Torres, A., Paz-Maldonado, L. M. T., Silva-Ramírez, A. S., Bañuelos-Hernández, B., Martínez-Salgado, J. L., \& Soria-Guerra, R. E. (2014). Over-expression of Dof-type transcription factor increases lipid production in Chlamydomonas reinhardtii. Journal of Biotechnology, 184, 27-38.

Ikehata, H., \& Ono, T. (2011). The Mechanisms of UV Mutagenesis. Journal of Radiation Research, 52(2), 115-125.

Inukai, S., Kock, K. H., \& Bulyk, M. L. (2017). Transcription factor-DNA binding: beyond binding site motifs. Current Opinion in Genetics \& Development, 43, 110-119.

Iskandarov, U., Sitnik, S., Shtaida, N., Didi-Cohen, S., Leu, S., KhozinGoldberg, I., Cohen, Z., \& Boussiba, S. (2016). Cloning and characterization of a GPAT-like gene from the microalga Lobosphaera incisa (Trebouxiophyceae): overexpression in Chlamydomonas reinhardtii enhances TAG production. Journal of Applied Phycology, 28(2), 907-919.

Iwai, M., Hori, K., Sasaki-Sekimoto, Y., Shimojima, M., \& Ohta, H. (2015). Manipulation of oil synthesis in Nannochloropsis strain NIES-2145 with a phosphorus starvation-inducible promoter from Chlamydomonas reinhardtii. Frontiers in Microbiology, 6, 912.

Iwai, M., Ikeda, K., Shimojima, M., \& Ohta, H. (2014). Enhancement of extraplastidic oil synthesis in Chlamydomonas reinhardtii using a type-2 diacylglycerol acyltransferase with a phosphorus starvation-inducible promoter. Plant Biotechnology Journal, 12(6), 808-819.

Jacquier, N., Mishra, S., Choudhary, V., \& Schneiter, R. (2013). Expression of oleosin and perilipins in yeast promotes formation of lipid droplets from the endoplasmic reticulum. Journal of Cell Science, 126(Pt 22), 5198209.

Janssen, J. H., Driessen, J. L. S. P., Lamers, P. P., Wijffels, R. H., \& Barbosa, M. J. (2018). Effect of initial biomass-specific photon supply rate on fatty acid accumulation in nitrogen depleted Nannochloropsis gaditana under simulated outdoor light conditions. Algal Research, 35, 595-601.

Jarvis, E. E., Dunahay, T. G., \& Brown, L. M. (1992). DNA nucleoside composition and methylation in several species of microalgae. Journal of Phycology, 28(3), 356-362. 
Jeon, K., Suresh, A., \& Kim, Y. C. (2013). Highly efficient molecular delivery into Chlamydomonas reinhardtii by electroporation. Korean Journal of Chemical Engineering, 30(8), 1626-1630.

Jeon, S., Lim, J. M., Lee, H. G., Shin, S. E., Kang, N. K., Park, Y. Il, Oh, H. M., Jeong, W. J., Jeong, B. R., \& Chang, Y. K. (2017). Current status and perspectives of genome editing technology for microalgae. Biotechnology for Biofuels, 10, 267.

Jeong, J., Baek, K., Kirst, H., Melis, A., \& Jin, E. S. (2017). Loss of CpSRP54 function leads to a truncated light-harvesting antenna size in Chlamydomonas reinhardtii. Biochimica et Biophysica Acta Bioenergetics, 1858(1), 45-55.

Jiang, W., Brueggeman, A. J., Horken, K. M., Plucinak, T. M., \& Weeks, D. P. (2014). Successful transient expression of Cas9 and single guide RNA genes in Chlamydomonas reinhardtii. Eukaryotic Cell, 13(11), 14651469.

Jinturkar, K. A., Rathi, M. N., \& Misra, A. (2011). Gene delivery using physical methods. In Challenges in Delivery of Therapeutic Genomics and Proteomics (pp. 83-126). Elsevier, Amsterdam.

Kang, N. K., Jeon, S., Kwon, S., Koh, H. G., Shin, S. E., Lee, B., Choi, G. G., Yang, J. W., Jeong, B. R., \& Chang, Y. K. (2015). Effects of overexpression of a bHLH transcription factor on biomass and lipid production in Nannochloropsis salina. Biotechnology for Biofuels, 8(1), 200.

Kang, N. K., Kim, E. K., Kim, Y. U., Lee, B., Jeong, W. J., Jeong, B. R., \& Chang, Y. K. (2017). Increased lipid production by heterologous expression of AtWRI1 transcription factor in Nannochloropsis salina. Biotechnology for Biofuels, 10(1), 231.

Kang, S., Kim, K.-H., \& Kim, Y.-C. (2015). A novel electroporation system for efficient molecular delivery into Chlamydomonas reinhardtii with a 3dimensional microelectrode. Scientific Reports, 5, 15835.

Karas, B. J., Diner, R. E., Lefebvre, S. C., McQuaid, J., Phillips, A. P. R., Noddings, C. M., ... Weyman, P. D. (2015). Designer diatom episomes delivered by bacterial conjugation. Nature Communications, 6, 6925.

Kasai, Y., Tsukahara, T., Ikeda, F., Ide, Y., \& Harayama, S. (2018). Metabolic engineering using iterative self-cloning to improve lipid productivity in Coccomyxa. Scientific Reports, 8(1), 1-11.

Kato, M., Sakai, M., Adachi, K., Ikemoto, H., \& Sano, H. (1996). Distribution of betaine lipids in marine algae. Phytochemistry, 42(5), 1341-1345.

Kaye, Y., Grundman, O., Leu, S., Zarka, A., Zorin, B., Didi-Cohen, S., Khozin- 
Goldberg, I., \& Boussiba, S. (2015). Metabolic engineering toward enhanced LC-PUFA biosynthesis in Nannochloropsis oceanica: Overexpression of endogenous $\delta 12$ desaturase driven by stress-inducible promoter leads to enhanced deposition of polyunsaturated fatty acids in TAG. Algal Research, 11, 387-398.

Khan, M. I., Shin, J. H., \& Kim, J. D. (2018). The promising future of microalgae: current status, challenges, and optimization of a sustainable and renewable industry for biofuels, feed, and other products. Microbial cell factories, $17(1), 36$.

Khozin-Goldberg, I., Iskandarov, U., \& Cohen, Z. (2011). LC-PUFA from photosynthetic microalgae: occurrence, biosynthesis, and prospects in biotechnology. Applied microbiology and biotechnology, 91(4), 905-915.

Kienesberger, P. C., Oberer, M., Lass, A., \& Zechner, R. (2009). Mammalian patatin domain containing proteins: a family with diverse lipolytic activities involved in multiple biological functions. Journal of Lipid Research, 50 Suppl(Suppl), S63-68.

Kilian, O., Benemann, C. S. E., Niyogi, K. K., \& Vick, B. (2011). High-efficiency homologous recombination in the oil-producing alga Nannochloropsis $s p$. Proceedings of the National Academy of Sciences of the United States of America, 108(52), 21265-21269.

Kim, J., Kwak, H. S., Sim, S. J., \& Jin, E. S. (2019). Overexpression of malic enzyme isoform 2 in Chlamydomonas reinhardtii PTS42 increases lipid production. Bioresource Technology Reports, 7, 100239.

Kindle, K. L. (1990). High-frequency nuclear transformation of Chlamydomonas reinhardtii. Proceedings of the National Academy of Sciences of the United States of America, 87(3), 1228-1232.

Kindle, K. L., Schnell, R. A., Fernández, E., \& Lefebvre, P. A. (1989). Stable nuclear transformation of Chlamydomonas using the Chlamydomonas gene for nitrate reductase. The Journal of cell biology, 109(6 Pt 1), 25892601.

Kindle, Karen L. (1998). High-frequency nuclear transformation of Chlamydomonas reinhardtii. Methods in Enzymology, 297, 27-38.

Kindle, Karen L., Richards, K. L., \& Stern, D. B. (1991). Engineering the chloroplast genome: Techniques and capabilities for chloroplast transformation in Chlamydomonas reinhardtii. Proceedings of the National Academy of Sciences of the United States of America, 88(5), 1721-1725.

Kirst, H., Garcia-Cerdan, J. G., Zurbriggen, A., Ruehle, T., \& Melis, A. (2012). Truncated photosystem chlorophyll antenna size in the green microalga Chlamydomonas reinhardtii upon deletion of the TLA3-CpSRP43 gene. 
Plant Physiology, 160(4), 2251-2260.

Klaitong, P., Fa-aroonsawat, S., \& Chungjatupornchai, W. (2017). Accelerated triacylglycerol production and altered fatty acid composition in oleaginous microalga Neochloris oleoabundans by overexpression of diacylglycerol acyltransferase 2. Microbial Cell Factories, 16(1), 61.

Koh, H.-J., Lee, S.-M., Son, B.-G., Lee, S.-H., Ryoo, Z. Y., Chang, K.-T., Park, J.-W., Park, D.-C., Song, B. J., Veech, R. L., Song, H., \& Huh, T.-L. (2004). Cytosolic NADP+-dependent isocitrate dehydrogenase plays a key role in lipid metabolism. The Journal of Biological Chemistry, 279(38), 3996874.

Koh, H. G., Kang, N. K., Jeon, S., Shin, S.-E., Jeong, B., \& Chang, Y. K. (2019). Heterologous synthesis of chlorophyll b in Nannochloropsis salina enhances growth and lipid production by increasing photosynthetic efficiency. Biotechnology for Biofuels, 12(1), 122.

Kong, F., Romero, I.T., Warakanont, J., \& Li-Beisson, Y. (2018). Lipid catabolism in microalgae. New Phytologist, 218, 1340-1348.

Korkhovoy, V., Tsarenko, P., Blume, Y. (2016). Genetically engineered microalgae for enhanced biofuel production. Current Biotechnology, 5, 256-265.

Kong, F., Liang, Y., Légeret, B., Beyly-Adriano, A., Blangy, S., Haslam, R. P., Napier, J. A., Beisson, F., Peltier, G., \& Li-Beisson, Y. (2017). Chlamydomonas carries out fatty acid $\beta$-oxidation in ancestral peroxisomes using a bona fide acyl-CoA oxidase. The Plant Journal, 90(2), 358-371.

Kong, F., Romero, I. T., Warakanont, J., \& Li-Beisson, Y. (2018). Lipid catabolism in microalgae. New Phytologist, 218(4), 1340-1348.

Koo, J., Park, D., \& Kim, H. (2013). Expression of bovine lactoferrin N-lobe by the green alga, Chlorella vulgaris. Algae, 28(4), 379-387.

Kruger, N. J., \& von Schaewen, A. (2003). The oxidative pentose phosphate pathway: structure and organisation. Current Opinion in Plant Biology, 6(3), 236-246.

Kumar, G., Shekh, A., Jakhu, S., Sharma, Y., Kapoor, R., \& Sharma, T. R. (2020). Bioengineering of Microalgae: Recent Advances, Perspectives, and Regulatory Challenges for Industrial Application. Frontiers in bioengineering and biotechnology, 8, 914.

Kumar, S. V., Misquitta, R. W., Reddy, V. S., Rao, B. J., \& Rajam, M. V. (2004). Genetic transformation of the green alga - Chlamydomonas reinhardtii by Agrobacterium tumefaciens. Plant Science, 166(3), 731-738. 
Kumari, M., Schoiswohl, G., Chitraju, C., Paar, M., Cornaciu, I., Rangrez, A. Y., ... Zechner, R. (2012). Adiponutrin functions as a nutritionally regulated lysophosphatidic acid acyltransferase. Cell Metabolism, 15(5), 691-702.

Kumari, S., Singh, P., Gupta, S.K., Kumar, S. (2015). Genetic engineering tools for enhancing lipid production in microalgae. In: Singh B., Bauddh K., Bux F. (eds) Algae and environmental sustainability. Developments in applied phycology, vol 7. Springer, New Delhi.

Kwon, S., Kang, N. K., Koh, H. G., Shin, S. E., Lee, B., Jeong, B. R., \& Chang, Y. K. (2018). Enhancement of biomass and lipid productivity by overexpression of a bZIP transcription factor in Nannochloropsis salina. Biotechnology and Bioengineering, 115(2), 331-340.

La Russa, M., Bogen, C., Uhmeyer, A., Doebbe, A., Filippone, E., Kruse, O., \& Mussgnug, J. H. (2012). Functional analysis of three type-2 DGAT homologue genes for triacylglycerol production in the green microalga Chlamydomonas reinhardtii. Journal of Biotechnology, 162(1), 13-20.

Lacroix, B., \& Citovsky, V. (2016). Transfer of DNA from Bacteria to Eukaryotes. mBio, 7(4), e00863-16.

Lam, A. J., St-Pierre, F., Gong, Y., Marshall, J. D., Cranfill, P. J., Baird, M. A., McKeown, M. R., Wiedenmann, J., Davidson, M. W., Schnitzer, M. J., Tsien, R. Y., \& Lin, M. Z. (2012). Improving FRET dynamic range with bright green and red fluorescent proteins. Nature Methods, 9(10), 10051012.

Lauersen, K. J., Kruse, O., \& Mussgnug, J. H. (2015). Targeted expression of nuclear transgenes in Chlamydomonas reinhardtii with a versatile, modular vector toolkit. Applied Microbiology and Biotechnology, 99(8), 3491-3503.

Lauritano, C., Ferrante, M. I., \& Rogato, A. (2019). Marine Natural Products from Microalgae: An -Omics Overview. Marine drugs, 17(5), 269.

Lee, B., Choi, G. G., Choi, Y. E., Sung, M., Park, M. S., \& Yang, J. W. (2014). Enhancement of lipid productivity by ethyl methane sulfonate-mediated random mutagenesis and proteomic analysis in Chlamydomonas reinhardtii. Korean Journal of Chemical Engineering, 31(6), 1036-1042.

Lee, J., Yamaoka, Y., Kong, F., Cagnon, C., Beyly-Adriano, A., Jang, S., Gao, P., Kang, B.H., Li-Beisson, Y., \& Lee, Y. (2020). The phosphatidylethanolamine-binding protein DTH1 mediates degradation of lipid droplets in Chlamydomonas reinhardtii. Proceedings of the National Academy of Sciences of the United States of America, 117, 23131-23139.

Lee, S. M., Koh, H.-J., Park, D.-C., Song, B. J., Huh, T.-L., \& Park, J.-W. (2002). Cytosolic NADP+-dependent isocitrate dehydrogenase status modulates 
oxidative damage to cells. Free Radical Biology and Medicine, 32(11), 1185-1196.

Lehmuskero, A., Skogen Chauton, M., \& Boström, T. (2018). Light and photosynthetic microalgae: A review of cellular- and molecular-scale optical processes. Progress in Oceanography, 168, 43-56.

León-Bañares, R., González-Ballester, D., Galván, A., \& Fernández, E. (2004). Transgenic microalgae as green cell-factories. Trends in biotechnology, 22(1), 45-52.

Li-Beisson, Y., Kong, F., Wang, P., Lee, Y., \& Kang, B.H. (2021). The disassembly of lipid droplets in Chlamydomonas. New Phytologist, 231, 1359-1364.

Li-Beisson, Y., Nakamura, Y., \& Harwood, J. (2016). Lipids: From chemical structures, biosynthesis, and analyses to industrial applications. Subcellular Biochemistry, 86, 1-18.

Li-Beisson, Y., Thelen, J. J., Fedosejevs, E., \& Harwood, J. L. (2019). The lipid biochemistry of eukaryotic algae. Progress in lipid research, 74, 31-68.

Li, D. W., Balamurugan, S., Yang, Y. F., Zheng, J. W., Huang, D., Zou, L. G., Yang, W. D., Liu, J. S., Guan, Y., \& Li, H. Y. (2019). Transcriptional regulation of microalgae for concurrent lipid overproduction and secretion. Science Advances, 5(1), eaau3795.

Li, D. W., Cen, S. Y., Liu, Y. H., Balamurugan, S., Zheng, X. Y., Alimujiang, A., Yang, W. D., Liu, J. S., \& Li, H. Y. (2016). A type 2 diacylglycerol acyltransferase accelerates the triacylglycerol biosynthesis in heterokont oleaginous microalga Nannochloropsis oceanica. Journal of Biotechnology, $229,65-71$.

Li, J. Z., Huang, Y., Karaman, R., Ivanova, P. T., Brown, H. A., Roddy, T., Castro-Perez, J., Cohen, J. C., \& Hobbs, H. H. (2012). Chronic overexpression of PNPLA3I148M in mouse liver causes hepatic steatosis. The Journal of Clinical Investigation, 122(11), 4130-4144.

Li, N. N., Chen, L., Li, X. H., Li, Q., Zhang, W. B., Takechi, K., Takano, H., \& Lin, X. F. (2017). Overexpression of UDP-glucose dehydrogenase from Larix gmelinii enhances growth and cold tolerance in transgenic Arabidopsis thaliana. Biologia Plantarum, 61(1), 95-105.

Li, Yanqun, Horsman, M., Wang, B., Wu, N., \& Lan, C. Q. (2008). Effects of nitrogen sources on cell growth and lipid accumulation of green alga Neochloris oleoabundans. Applied Microbiology and Biotechnology, 81(4), 629-636.

Li, Yantao, Han, D., Hu, G., Dauvillee, D., Sommerfeld, M., Ball, S., \& Hu, Q. 
(2010). Chlamydomonas starchless mutant defective in ADP-glucose pyrophosphorylase hyper-accumulates triacylglycerol. Metabolic Engineering, 12(4), 387-391.

Li, Z., Meng, T., Ling, X., Li, J., Zheng, C., Shi, Y., Chen, Z., Li, Z., Li, Q., Lu, Y., \& He, N. (2018). Overexpression of malonyl-CoA: ACP transacylase in Schizochytrium sp. to improve polyunsaturated fatty acid production. Journal of Agricultural and Food Chemistry, 66(21), 5382-5391.

Lichtenthaler, H. K. (1987). Chlorophylls and carotenoids: pigments of photosynthetic biomembranes. Methods in Enzymology, 148, 350-382.

Lim, D. K. Y., Garg, S., Timmins, M., Zhang, E. S. B., Thomas-Hall, S. R., Schuhmann, H., Li, Y., \& Schenk, P. M. (2012). Isolation and evaluation of oil-producing microalgae from subtropical coastal and brackish waters. PLOS ONE, 7(7), e40751.

Lin, H., \& Lee, Y. K. (2017). Genetic engineering of medium-chain-length fatty acid synthesis in Dunaliella tertiolecta for improved biodiesel production. Journal of Applied Phycology, 29(6), 2811-2819.

Lin, H., Shen, H., \& Lee, Y. K. (2018). Cellular and Molecular Responses of Dunaliella tertiolecta by Expression of a Plant Medium Chain Length Fatty Acid Specific Acyl-ACP Thioesterase. Frontiers in microbiology, 9, 619.

Lin, W. R., Tan, S. I., Hsiang, C. C., Sung, P. K., \& Ng, I. S. (2019). Challenges and opportunity of recent genome editing and multi-omics in cyanobacteria and microalgae for biorefinery. Bioresource technology, 291, 121932.

Liu, X. Y., Teng, Y. B., Li, B., \& Meng, Q. W. (2013). Enhancement of lowtemperature tolerance in transgenic tomato plants overexpressing Lefad7 through regulation of trienoic fatty acids. Photosynthetica, 51(2), 238244.

Liu, Z., Chen, O., Wall, J., Zheng, M., Zhou, Y., Wang, L., Vaseghi, H. R., Qian, L., \& Liu, J. (2017). Systematic comparison of 2A peptides for cloning multi-genes in a polycistronic vector. Scientific reports, 7(1), 2193.

Lum, K. K., Kim, J., \& Lei, X. G. (2013). Dual potential of microalgae as a sustainable biofuel feedstock and animal feed. Journal of animal science and biotechnology, 4(1), 53.

Macfie, S. M., Tarmohamed, Y., \& Welbourn, P. M. (1994). Effects of cadmium, cobalt, copper, and nickel on growth of the green alga Chlamydomonas reinhardtii: The influences of the cell wall and $\mathrm{pH}$. Archives of Environmental Contamination and Toxicology, 27(4), 454-458. 
Madhavan, S., Andreo, C. S., Maurino, V. G., \& O'Leary, M. H. (2002). In situ localization of NADP-malic enzyme in bundle sheath cells and leaf carbon isotope fractionation in two $\mathrm{C}_{4}$ grasses. International Journal of Plant Sciences, 157, 1.

Maeo, K., Tokuda, T., Ayame, A., Mitsui, N., Kawai, T., Tsukagoshi, H., Ishiguro, S., \& Nakamura, K. (2009). An AP2-type transcription factor, WRINKLED1, of Arabidopsis thaliana binds to the AW-box sequence conserved among proximal upstream regions of genes involved in fatty acid synthesis. The Plant Journal, 60(3), 476-487.

Majidian, P., Tabatabaei, M., Zeinolabedini, M., Naghshbandi, M. P., \& Chisti, Y. (2018). Metabolic engineering of microorganisms for biofuel production. Renewable and Sustainable Energy Reviews, 82, 3863-3885.

Manandhar-Shrestha, K., \& Hildebrand, M. (2015). Characterization and manipulation of a DGAT2 from the diatom Thalassiosira pseudonana: Improved TAG accumulation without detriment to growth, and implications for chloroplast TAG accumulation. Algal Research, 12, 239248.

Mandal, S., \& Mallick, N. (2009). Microalga Scenedesmus obliquus as a potential source for biodiesel production. Applied Microbiology and Biotechnology, 84(2), 281-291.

Martínez-Rivas, J. M., \& Vega, J. M. (1994). Studies on the isoforms of isocitrate dehydrogenase from Chlamydomonas reinhardtii. Journal of Plant Physiology, 143(2), 129-134.

Masojídek, J., Torzillo, G., \& Koblížek, M. (2013). Photosynthesis in microalgae. In Handbook of Microalgal Culture: Applied Phycology and Biotechnology (pp. 21-36).

Matsumoto, T. K., \& Gonsalves, D. (2012). Biolistic and other nonAgrobacterium technologies of plant transformation. In Plant Biotechnology and Agriculture (pp. 117-129).

Medipally, S. R., Yusoff, F. M., Banerjee, S., \& Shariff, M. (2015). Microalgae as sustainable renewable energy feedstock for biofuel production. BioMed Research International, 2015, 519513.

Merchant, S. S., Prochnik, S. E., Vallon, O., Harris, E. H., Karpowicz, S. J., Witman, G. B., ... Zhou, K. (2007). The Chlamydomonas genome reveals the evolution of key animal and plant functions. Science, 318(5848), 245251.

Miranda, J. R., Passarinho, P. C., \& Gouveia, L. (2012). Bioethanol production from Scenedesmus obliquus sugars: The influence of photobioreactors and culture conditions on biomass production. Applied Microbiology and 
Biotechnology, 96(2), 555-564.

Moellering, E. R., Miller, R., \& Benning, C. (2009). Molecular genetics of lipid metabolism in the model green alga Chlamydomonas reinhardtii (pp. 139-155). Springer, Dordrecht.

Moody, J. W., McGinty, C. M., \& Quinn, J. C. (2014). Global evaluation of biofuel potential from microalgae. Proceedings of the National Academy of Sciences of the United States of America, 111(23), 8691-8696.

Moriyama, T., Toyoshima, M., Saito, M., Wada, H., \& Sato, N. (2018). Revisiting the algal "chloroplast lipid droplet": the absence of an entity that is unlikely to exist. Plant Physiology, 176(2), 1519-1530.

Morris, S. N. S., \& Olzmann, J. A. (2019). A tense situation: maintaining er homeostasis during lipid droplet budding. Developmental Cell, 50(1), 12.

Muñoz, C. F., de Jaeger, L., Sturme, M. H. J., Lip, K. Y. F., Olijslager, J. W. J., Springer, J., Wolbert, E. J. H., Martens, D. E., Eggink, G., Weusthuis, R. A., \& Wijffels, R. H. (2018). Improved DNA/protein delivery in microalgae - A simple and reliable method for the prediction of optimal electroporation settings. Algal Research, 33, 448-455.

Muñoz, C. F., Weusthuis, R. A., D'Adamo, S., \& Wijffels, R. H. (2019). Effect of single and combined expression of lysophosphatidic acid acyltransferase, glycerol-3-phosphate acyltransferase, and diacylglycerol acyltransferase on lipid accumulation and composition in Neochloris oleoabundans. Frontiers in Plant Science, 10, 1573.

Murakami, H., Nobusawa, T., Hori, K., Shimojima, M., \& Ohta, H. (2018). Betaine lipid is crucial for adapting to low temperature and phosphate deficiency in Nannochloropsis. Plant Physiology, 177(1), 181-193.

Muto, M., Tanaka, M., Liang, Y., Yoshino, T., Matsumoto, M., \& Tanaka, T. (2015). Enhancement of glycerol metabolism in the oleaginous marine diatom Fistulifera solaris JPCC DA0580 to improve triacylglycerol productivity. Biotechnology for Biofuels, 8(1), 4.

Naduthodi, M., Claassens, N. J., D'Adamo, S., van der Oost, J., \& Barbosa, M. J. (2021). Synthetic Biology Approaches To Enhance Microalgal Productivity. Trends in biotechnology, S0167-7799(21)00004-4.

Naghshbandi, M. P., Tabatabaei, M., Aghbashlo, M., Aftab, M. N., \& Iqbal, I. (2020). Metabolic Engineering of Microalgae for Biofuel Production. Methods in molecular biology (Clifton, N.J.), 1980, 153-172.

Nations, F. and A. O. of the U. (2016). The State of World Fisheries and Aquaculture 2016. UN. 
Ng, I. S., Tan, S. I., Kao, P. H., Chang, Y. K., \& Chang, J. S. (2017). Recent Developments on Genetic Engineering of Microalgae for Biofuels and BioBased 10.1002/biot.201600644.

journal, 12(10),

Ngan, C. Y., Wong, C.-H., Choi, C., Yoshinaga, Y., Louie, K., Jia, J., ... Wei, C.L. (2015). Lineage-specific chromatin signatures reveal a regulator of lipid metabolism in microalgae. Nature Plants, 1(8), 15107.

Niu, Y.-F., Zhang, M.-H., Li, D.-W., Yang, W.-D., Liu, J.-S., Bai, W.-B., \& Li, H.Y. (2013). Improvement of neutral lipid and polyunsaturated fatty acid biosynthesis by overexpressing a type 2 diacylglycerol acyltransferase in marine diatom Phaeodactylum tricornutum. Marine Drugs, 11(11), 45584569.

Niu, Y. F., Wang, X., Hu, D. X., Balamurugan, S., Li, D. W., Yang, W. D., Liu, J. S., \& Li, H. Y. (2016). Molecular characterization of a glycerol-3phosphate acyltransferase reveals key features essential for triacylglycerol production in Phaeodactylum tricornutum. Biotechnology for Biofuels, 9(1).

Norashikin, M. N., Loh, S. H., Aziz, A., \& Cha, T. S. (2018). Metabolic engineering of fatty acid biosynthesis in Chlorella vulgaris using an endogenous omega- 3 fatty acid desaturase gene with its promoter. Algal Research, 31, 262-275.

Nymark, M., Sharma, A. K., Sparstad, T., Bones, A. M., \& Winge, P. (2016). A CRISPR/Cas9 system adapted for gene editing in marine algae. Scientific Reports, 6(1), 1-6.

Oka, T., \& Jigami, Y. (2006). Reconstruction of de novo pathway for synthesis of UDP-glucuronic acid and UDP-xylose from intrinsic UDP-glucose in Saccharomyces cerevisiae. FEBS Journal, 273(12), 2645-2657.

Olzmann, J. A., \& Carvalho, P. (2019). Dynamics and functions of lipid droplets. Nature Reviews Molecular Cell Biology, 20(3), 137-155.

Ortiz-Matamoros, M. F., Villanueva, M. A., \& Islas-Flores, T. (2018). Genetic transformation of cell-walled plant and algae cells: delivering DNA through the cell wall. Briefings in Functional Genomics, 17(1), 26-33.

Park, S., Nguyen, T., \& Jin, E. (2019). Improving lipid production by strain development in microalgae: Strategies, challenges and perspectives. Bioresource technology, 292, 121953.

Peng, K. T., Zheng, C. N., Xue, J., Chen, X. Y., Yang, W. D., Liu, J. S., Bai, W., $\&$ Li, H. Y. (2014). Delta 5 fatty acid desaturase upregulates the synthesis of polyunsaturated fatty acids in the marine diatom Phaeodactylum tricornutum. Journal of Agricultural and Food Chemistry, 62(35), 8773- 
8776.

Peviani, A., Lastdrager, J., Hanson, J., \& Snel, B. (2016). The phylogeny of C/S1 bZIP transcription factors reveals a shared algal ancestry and the pre-angiosperm translational regulation of $\mathrm{S} 1$ transcripts. Scientific Reports, 6, 30444.

Pieper, S., Unterieser, I., Mann, F., \& Mischnick, P. (2012). A new arabinomannan from the cell wall of the chlorococcal algae Chlorella vulgaris. Carbohydrate Research, 352, 166-176.

Pingitore, P., Pirazzi, C., Mancina, R. M., Motta, B. M., Indiveri, C., Pujia, A., Montalcini, T., Hedfalk, K., \& Romeo, S. (2014). Recombinant PNPLA3 protein shows triglyceride hydrolase activity and its I148M mutation results in loss of function. Biochimica et Biophysica Acta (BBA) - Molecular and Cell Biology of Lipids, 1841(4), 574-580.

Poliner, E., Pulman, J. A., Zienkiewicz, K., Childs, K., Benning, C., \& Farré, E. M. (2018). A toolkit for Nannochloropsis oceanica CCMP1779 enables gene stacking and genetic engineering of the eicosapentaenoic acid pathway for enhanced long-chain polyunsaturated fatty acid production. Plant Biotechnology Journal, 16(1), 298-309.

Poliner, E., Takeuchi, T., Du, Z. Y., Benning, C., \& Farré, E. M. (2018). Nontransgenic marker-free gene disruption by an episomal CRISPR system in the oleaginous microalga, Nannochloropsis oceanica CCMP1779. ACS Synthetic Biology, 7(4), 962-968.

Pulz, O., \& Gross, W. (2004). Valuable products from biotechnology of microalgae. Applied microbiology and biotechnology, 65(6), 635-648.

Radakovits, R., Eduafo, P. M., \& Posewitz, M. C. (2011). Genetic engineering of fatty acid chain length in Phaeodactylum tricornutum. Metabolic Engineering, 13(1), 89-95.

Radakovits, R., Jinkerson, R. E., Darzins, A., \& Posewitz, M. C. (2010). Genetic engineering of algae for enhanced biofuel production. Eukaryotic cell, 9(4), 486-501.

Radakovits, R., Jinkerson, R. E., Fuerstenberg, S. I., Tae, H., Settlage, R. E., Boore, J. L., \& Posewitz, M. C. (2012). Draft genome sequence and genetic transformation of the oleaginous alga Nannochloropis gaditana. Nature Communications, 3(1), 1-11.

Ramazanov, A., Ramazanov, Z. (2006). Isolation and characterization of a starchless mutant of Chlorella pyrenoidosa STL-PI with a high growth rate, and high protein and polyunsaturated fatty acid content. Phycological Research, 54, 255-259. 
Ramesh, V. M., Bingham, S. E., \& Webber, A. N. (2011). A simple method for chloroplast transformation in Chlamydomonas reinhardtii. Methods in Molecular Biology (Clifton, N.J.), 684, 313-320.

Ran, W., Wang, H., Liu, Y., Qi, M., Xiang, Q., Yao, C., Zhang, Y., \& Lan, X. (2019). Storage of starch and lipids in microalgae: Biosynthesis and manipulation by nutrients. Bioresource technology, 291, 121894.

Rasala, B. A., Lee, P. A., Shen, Z., Briggs, S. P., Mendez, M., \& Mayfield, S. P. (2012). Robust expression and secretion of xylanase1 in Chlamydomonas reinhardtii by fusion to a selection gene and processing with the FMDV $2 A$ peptide. PLOS ONE, 7(8).

Ratledge, C. (2014). The role of malic enzyme as the provider of NADPH in oleaginous microorganisms: a reappraisal and unsolved problems. Biotechnology Letters, 36(8), 1557-1568.

Remmers, I. M., Wijffels, R. H., Barbosa, M. J., \& Lamers, P. P. (2018). Can We Approach Theoretical Lipid Yields in Microalgae?.Trends in biotechnology, 36(3), 265-276.

Rengel, R., Smith, R. T., Haslam, R. P., Sayanova, O., Vila, M., \& León, R. (2018). Overexpression of acetyl-CoA synthetase (ACS) enhances the biosynthesis of neutral lipids and starch in the green microalga Chlamydomonas reinhardtii. Algal Research, 31, 183-193.

Richard, D., Kefi, K., Barbe, U., Bausero, P., \& Visioli, F. (2008). Polyunsaturated fatty acids as antioxidants. Pharmacological Research, 57(6), 451-455.

Riekhof, W. R., Sears, B. B., \& Benning, C. (2005). Annotation of genes involved in glycerolipid biosynthesis in Chlamydomonas reinhardtii: Discovery of the betaine lipid synthase BTA1Cr. Eukaryotic Cell, 4(2), 242-252.

Ruiz, J., Olivieri, G., De Vree, J., Bosma, R., Willems, P., Reith, J. H., Eppink, M. H. M., Kleinegris, D. M. M., Wijffels, R. H., \& Barbosa, M. J. (2016). Towards industrial products from microalgae. Energy and Environmental Science, 9, 3036-3043.

Sanitha, M., Radha, S., Fatima, A. A., Devi, S. G., \& Ramya, M. (2014). Agrobacterium-mediated transformation of three freshwater microalgal strains. Polish Journal of Microbiology, 63(4), 387-392.

Sathasivam, R., \& Ki, J. S. (2018). A Review of the Biological Activities of Microalgal Carotenoids and Their Potential Use in Healthcare and Cosmetic Industries. Marine drugs, 16(1), 26.

Sato, N., \& Moriyama, T. (2018). Photosynthesis. In Cyanidioschyzon merolae: A New Model Eukaryote for Cell and Organelle Biology (pp. 263-281). 
Sauer, U., Canonaco, F., Heri, S., Perrenoud, A., \& Fischer, E. (2004). The soluble and membrane-bound transhydrogenases UdhA and PntAB have divergent functions in NADPH metabolism of Escherichia coli. Journal of Biological Chemistry, 279(8), 6613-6619.

Schmid-Siegert, E., Stepushenko, O., Glauser, G., \& Farmer, E. E. (2016). Membranes as structural antioxidants recycling of malondialdehyde to its source in oxidation-Sensitive chloroplast fatty aciDS. Journal of Biological Chemistry, 291(25), 13005-13013.

Schroda, M., Vallon, O., Wollman, F. A., \& Beck, C. F. (1999). A chloroplasttargeted heat shock protein 70 (HSP70) contributes to the photoprotection and repair of photosystem II during and after photoinhibition. Plant Cell, 11(6), 1165-1178.

Shechter, I., Dai, P., Huo, L., \& Guan, G. (2003). IDH1 gene transcription is sterol regulated and activated by SREBP-1a and SREBP-2 in human hepatoma HepG2 cells: evidence that IDH1 may regulate lipogenesis in hepatic cells. Journal of lipid research, 44(11), 2169-2180.

Sheehan, J., Dunahay, T., Benemann, J., \& Roessler, P. (1998). Look Back at the U.S. Department of Energy's Aquatic Species Program: Biodiesel from Algae; Close-Out Report. Golden, CO. Department of Energy.

Shi, H., Luo, X., Wu, R., \& Yue, X. (2018). Production of eicosapentaenoic acid by application of a delta- 6 desaturase with the highest ALA catalytic activity in algae. Microbial Cell Factories, 17(1), 7.

Shimogawara, K., Fujiwara, S., Grossman, A., \& Usuda, H. (1998). Highefficiency transformation of Chlamydomonas reinhardtii by electroporation. Genetics, 148(4), 1821-1828.

Shin, S. E., Lim, J. M., Koh, H. G., Kim, E. K., Kang, N. K., Jeon, S., Kwon, S., Shin, W. S., Lee, B., Hwangbo, K., Kim, J., Ye, S. H., Yun, J. Y., Seo, H., Oh, H. M., Kim, K. J., Kim, J. S., Jeong, W. J., Chang, Y. K., \& Jeong, B. R. (2016). CRISPR/Cas9-induced knockout and knock-in mutations in Chlamydomonas reinhardtii. Scientific reports, 6, 27810.

Sivaramakrishnan, R., \& Incharoensakdi, A. (2017). Enhancement of lipid production in Scenedesmus $s p$. by UV mutagenesis and hydrogen peroxide treatment. Bioresource Technology, 235, 366-370.

Slattery, S. S., Diamond, A., Wang, H., Therrien, J. A., Lant, J. T., Jazey, T., Lee, K., Klassen, Z., Desgagné-Penix, I., Karas, B. J., \& Edgell, D. R. (2018). An expanded plasmid-based genetic toolbox enables Cas9 genome editing and stable maintenance of synthetic pathways in Phaeodactylum tricornutum. ACS Synthetic Biology, 7(2), 328-338.

Snyder, C. L., Yurchenko, O. P., Siloto, R. M. P., Chen, X., Liu, Q., Mietkiewska, 
E., \& Weselake, R. J. (2009). Acyltransferase action in the modification of seed oil biosynthesis. New Biotechnology, 26(1-2), 11-16.

Solovchenko, A. E. (2012). Physiological role of neutral lipid accumulation in eukaryotic microalgae under stresses. Russian Journal of Plant Physiology, 59, 167-176.

Song, Q.-X., Li, Q.-T., Liu, Y.-F., Zhang, F.-X., Ma, B., Zhang, W.-K., Man, W.Q., Du, W.-G., Wang, G.-D., Chen, S.-Y., \& Zhang, J.-S. (2013). Soybean GmbZIP123 gene enhances lipid content in the seeds of transgenic Arabidopsis plants. Journal of Experimental Botany, 64(14), 4329-4341.

Subramanian, S., Barry, A. N., Pieris, S., \& Sayre, R. T. (2013). Comparative energetics and kinetics of autotrophic lipid and starch metabolism in chlorophytic microalgae: Implications for biomass and biofuel production. Biotechnology for Biofuels, 6(1), 150.

Südfeld, C., Hubáček, M., Figueiredo, D., Naduthodi, M. I. S., van der Oost, J., Wijffels, R. H., Barbosa, M. J., \& D'Adamo, S. (2021). High-throughput insertional mutagenesis reveals novel targets for enhancing lipid accumulation in Nannochloropsis oceanica. Metabolic Engineering, 66, 239-258.

Sumiya, N., Kawase, Y., Hayakawa, J., Matsuda, M., Nakamura, M., Era, A., Tanaka, K., Kondo, A., Hasunuma, T., Imamura, S., \& Miyagishima, S. Y. (2015). Expression of cyanobacterial Acyl-ACP reductase elevates the triacylglycerol level in the red alga Cyanidioschyzon merolae. Plant and Cell Physiology, 56(10), 1962-1980.

Sun, X., Cao, Y., Xu, H., Liu, Y., Sun, J., Qiao, D., \& Cao, Y. (2014). Effect of nitrogen-starvation, light intensity and iron on triacylglyceride/carbohydrate production and fatty acid profile of Neochloris oleoabundans HK-129 by a two-stage process. Bioresource Technology, 155, 204-212.

Sun, X. M., Ren, L. J., Zhao, Q. Y., Ji, X. J., \& Huang, H. (2018). Microalgae for the production of lipid and carotenoids: A review with focus on stress regulation and adaptation. Biotechnology for Biofuels, 11, 272.

Suttangkakul, A., Sirikhachornkit, A., Juntawong, P., Puangtame, W., Chomtong, T., Srifa, S., Sathitnaitham, S., Dumrongthawatchai, W., Jariyachawalid, K., \& Vuttipongchaikij, S. (2019). Evaluation of strategies for improving the transgene expression in an oleaginous microalga Scenedesmus acutus. BMC Biotechnology, 19(1), 4.

Talebi, A. F., Tohidfar, M., Bagheri, A., Lyon, S. R., Salehi-Ashtiani, K., \& Tabatabaei, M. (2014). Manipulation of carbon flux into fatty acid biosynthesis pathway in Dunaliella salina using AccD and ME genes to enhance lipid content and to improve produced biodiesel quality. Biofuel 
Research Journal, 1(3), 91-97.

Tan, C., Qin, S., Zhang, Q., Jiang, P., \& Zhao, F. (2005). Establishment of a micro-particle bombardment transformation system for Dunaliella salina. Journal of Microbiology, 43(4), 361-365.

Tan, K. W. M., \& Lee, Y. K. (2017). Expression of the heterologous Dunaliella tertiolecta fatty acyl-ACP thioesterase leads to increased lipid production in Chlamydomonas reinhardtii. Journal of Biotechnology, 247, 60-67.

Tardif, M., Atteia, A., Specht, M., Cogne, G., Rolland, N., Brugière, S., Hippler, M., Ferro, M., Bruley, C., Peltier, G., Vallon, O., \& Cournac, L. (2012). PredAlgo: a new subcellular localization prediction tool dedicated to green algae. Molecular Biology and Evolution, 29(12), 3625-39.

Thiam, A. R., \& Beller, M. (2017). The why, when and how of lipid droplet diversity. Journal of cell science, $130(2), 315-324$.

Thiriet-Rupert, S., Carrier, G., Chénais, B., Trottier, C., Bougaran, G., Cadoret, J. P., Schoefs, B., \& Saint-Jean, B. (2016). Transcription factors in microalgae: Genome-wide prediction and comparative analysis. BMC Genomics, 17(1), 1-16.

Tsai, C.-H., Warakanont, J., Takeuchi, T., Sears, B. B., Moellering, E. R., \& Benning, C. (2014). The protein compromised hydrolysis of triacylglycerols 7 (cht7) acts as a repressor of cellular quiescence in Chlamydomonas. Proceedings of the National Academy of Sciences, 111(44), 15833-15838.

Úbeda-Mínguez, P., García-Maroto, F., \& Alonso, D. L. (2017). Heterologous expression of DGAT genes in the marine microalga Tetraselmis chui leads to an increase in TAG content. Journal of Applied Phycology, 29(4), 19131926.

Uranbey, S., Sevimay, C. S., Kaya, M. D., İpek, A., Sancak, C., Başalma, D., $\mathrm{Er}$, C., \& Özcan, S. (2005). Influence of different co-cultivation temperatures, periods and media on Agrobacterium tumefaciensmediated gene transfer. Biologia Plantarum, 49(1), 53-57.

Vazquez-Villegas, P., Torres-Acosta, M. A., Garcia-Echauri, S. A., AguilarYanez, J. M., Rito-Palomares, M., \& Ruiz-Ruiz, F. (2018). Genetic manipulation of microalgae for the production of bioproducts. Frontiers in Bioscience - Elite, 10(2), 254-275.

Vieler, A., Wu, G., Tsai, C. H., Bullard, B., Cornish, A. J., Harvey, C., ... Benning, C. (2012). Genome, functional gene annotation, and nuclear transformation of the heterokont oleaginous alga Nannochloropsis oceanica CCMP1779. PLoS Genetics, 8(11), e1003064. 
Vigeolas, H., \& Geigenberger, P. (2004). Increased levels of glycerol-3phosphate lead to a stimulation of flux into triacylglycerol synthesis after supplying glycerol to developing seeds of Brassica napus $L$. in planta. Planta, 219(5), 827-835.

Vila, M., Díaz-Santos, E., De La Vega, M., Rodriǵuez, H., Vargas, Á., \& León, R. (2012). Promoter trapping in microalgae using the antibiotic paromomycin as selective agent. Marine Drugs, 10(12), 2749-2765.

Voigt, J. (1988). The lithium-chloride-soluble cell-wall layers of Chlamydomonas reinhardii contain several immunologically related glycoproteins. Planta, 173(3), 373-384.

Vonlanthen, S., Dauvillée, D., \& Purton, S. (2015). Evaluation of novel starchdeficient mutants of Chlorella sorokiniana for hyper-accumulation of lipids. Algal Research, 12, 109-118.

Volk, S., Wang, M., \& Pickart, C. M. (2005). Chemical and genetic strategies for manipulating polyubiquitin chain structure. Methods in Enzymology, 399, 3-20.

Wang, Chaogang, Li, Y., Lu, J., Deng, X., Li, H., \& Hu, Z. (2018). Effect of overexpression of LPAAT and GPD1 on lipid synthesis and composition in green microalga Chlamydomonas reinhardtii. Journal of Applied Phycology, 30(3), 1711-1719.

Wang, Chinchin, \& Lan, C. Q. (2018). Effects of shear stress on microalgae - A review. Biotechnology Advances, 36(4), 986-1002.

Wang, D., Li, Y., Hu, X., Su, W., \& Zhong, M. (2015). Combined enzymatic and mechanical cell disruption and lipid extraction of green alga Neochloris oleoabundans. International Journal of Molecular Sciences, 16(4), 77077722.

Wang, H.-W., Zhang, B., Hao, Y.-J., Huang, J., Tian, A.-G., Liao, Y., Zhang, J.S., \& Chen, S.-Y. (2007). The soybean Dof-type transcription factor genes, GmDof4 and GmDof11, enhance lipid content in the seeds of transgenic Arabidopsis plants. The Plant Journal, 52(4), 716-729.

Wang, Q., Feng, Y., Lu, Y., Xin, Y., Shen, C., Wei, L., Liu, Y., Lv, N., Du, X., Zhu, W., Jeong, B., Xue, S., \& Xu, J. (2021). Manipulating fatty-acid profile at unit chain-length resolution in the model industrial oleaginous microalgae Nannochloropsis. Metabolic Engineering, 66, 157-166.

Wang, Q., Lu, Y., Xin, Y., Wei, L., Huang, S., \& Xu, J. (2016). Genome editing of model oleaginous microalgae Nannochloropsis spp. by CRISPR/Cas9. The Plant Journal, 88(6), 1071-1081.

Wang, X., Dong, H. P., Wei, W., Balamurugan, S., Yang, W. D., Liu, J. S., \& Li, 
H. Y. (2018). Dual expression of plastidial GPAT1 and LPAT1 regulates triacylglycerol production and the fatty acid profile in Phaeodactylum tricornutum 06 Biological Sciences 0601 Biochemistry and Cell Biology. Biotechnology for Biofuels, 11(1), 318.

Wang, X., Liu, S. F., Li, R. Y., Yang, W. D., Liu, J. S., Lin, C. S. K., Balamurugan, S., \& Li, H. Y. (2020). TAG pathway engineering via GPAT2 concurrently potentiates abiotic stress tolerance and oleaginicity in Phaeodactylum tricornutum. Biotechnology for Biofuels, 13(1), 160.

Wang, X., Liu, Y. H., Hu, D. X., Balamurugan, S., Lu, Y., Yang, W. D., Liu, J. S., \& Li, H. Y. (2015). Identification of a putative patatin-like phospholipase domain-containing protein 3 (PNPLA3) ortholog involved in lipid metabolism in microalga Phaeodactylum tricornutum. Algal Research, 12, 274-279.

Wang, X., Wei, W., Li, N. J., Yuan, W., Ding, Y., Yang, W. D., Liu, J. S., Balamurugan, S., \& Li, H. Y. (2018). Heterogeneous expression of human PNPLA3 triggers algal lipid accumulation and lipid droplet enlargement. Algal Research, 31, 276-281.

Wang, Y., Wang, F., Wang, R., Zhao, P., \& Xia, Q. (2015). 2A self-cleaving peptide-based multi-gene expression system in the silkworm Bombyx mori. Scientific Reports, 5, 16273.

Wei, H., Shi, Y., Ma, X., Pan, Y., Hu, H., Li, Y., Luo, M., Gerken, H., \& Liu, J. (2017). A type-I diacylglycerol acyltransferase modulates triacylglycerol biosynthesis and fatty acid composition in the oleaginous microalga, Nannochloropsis oceanica. Biotechnology for Biofuels, 10(1), 174.

Wei, L., Wang, Q., Xin, Y., Lu, Y., \& Xu, J. (2017). Enhancing photosynthetic biomass productivity of industrial oleaginous microalgae by overexpression of RuBisCO activase. Algal Research, 27, 366-375.

Wen, R., Wang, S., Xiang, D., Venglat, P., Shi, X., Zang, Y., Datla, R., Xiao, W., \& Wang, H. (2014). UBC13, an E2 enzyme for Lys63-linked ubiquitination, functions in root development by affecting auxin signaling and Aux/IAA protein stability. The Plant Journal, 80(3), 424-436.

Wijffels, R. H., \& Barbosa, M. J. (2010). An outlook on microalgal biofuels. Science, 329(5993), 796-799.

Wijffels, R. H., Kruse, O., \& Hellingwerf, K. J. (2013). Potential of industrial biotechnology with cyanobacteria and eukaryotic microalgae. Current opinion in biotechnology, 24(3), 405-413.

Wykoff, D. D., Grossman, A. R., Weeks, D. P., Usuda, H., \& Shimogawara, K. (1999). Psr1, a nuclear localized protein that regulates phosphorus metabolism in Chlamydomonas. Proceedings of the National Academy of 
Sciences of the United States of America, 96(26), 15336-41.

Wynn, J. P., Hamid, A. b. A., \& Ratledge, C. (1999). The role of malic enzyme in the regulation of lipid accumulation in filamentous fungi. Microbiology, 145(8), 1911-1917.

Xue, J., Balamurugan, S., Li, D. W., Liu, Y. H., Zeng, H., Wang, L., Yang, W. D., Liu, J. S., \& Li, H. Y. (2017). Glucose-6-phosphate dehydrogenase as a target for highly efficient fatty acid biosynthesis in microalgae by enhancing NADPH supply. Metabolic Engineering, 41, 212-221.

Xue, J., Chen, T., Zheng, J., Balamurugan, S., Liu, Y., Yang, W., Liu, J., \& Li, H. (2020). Glucose-6-phosphate dehydrogenase from the oleaginous microalga Nannochloropsis uncovers its potential role in promoting lipogenesis. Biotechnology Journal, 15(2), 1900135.

Xue, J., Niu, Y. F., Huang, T., Yang, W. D., Liu, J. S., \& Li, H. Y. (2015). Genetic improvement of the microalga Phaeodactylum tricornutum for boosting neutral lipid accumulation. Metabolic Engineering, 27, 1-9.

Xue, J., Wang, L., Zhang, L., Balamurugan, S., Li, D. W., Zeng, H., Yang, W. D., Liu, J. S., \& Li, H. Y. (2016). The pivotal role of malic enzyme in enhancing oil accumulation in green microalga Chlorella pyrenoidosa. Microbial Cell Factories, 15(1), 120.

Yamaoka, Y., Achard, D., Jang, S., Legéret, B., Kamisuki, S., Ko, D., SchulzRaffelt, M., Kim, Y., Song, W. Y., Nishida, I., Li-Beisson, Y., \& Lee, Y. (2016). Identification of a Chlamydomonas plastidial 2-lysophosphatidic acid acyltransferase and its use to engineer microalgae with increased oil content. Plant Biotechnology Journal, 14(11), 2158-2167.

Yamaoka, Y., Shin, S., Choi, B. Y., Kim, H., Jang, S., Kajikawa, M., Yamano, T., Kong, F., Légeret, B., Fukuzawa, H., Li-Beisson, Y., \& Lee, Y. (2019). The bZIP1 transcription factor regulates lipid remodeling and contributes to er stress management in Chlamydomonas reinhardtii. The Plant Cell, 31(5), 1127-1140.

Yan, J., Cheng, R., Lin, X., You, S., Li, K., Rong, H., \& Ma, Y. (2013). Overexpression of acetyl-CoA synthetase increased the biomass and fatty acid proportion in microalga Schizochytrium. Applied Microbiology and Biotechnology, 97(5), 1933-1939.

Yan, N., Fan, C., Chen, Y., \& Hu, Z. (2016). The potential for microalgae as bioreactors to produce pharmaceuticals. International Journal of Molecular Sciences, 17(6).

Yang, B., Liu, J., Ma, X., Guo, B., Liu, B., Wu, T., Jiang, Y., \& Chen, F. (2017). Genetic engineering of the Calvin cycle toward enhanced photosynthetic $\mathrm{CO}_{2}$ fixation in microalgae. Biotechnology for Biofuels, 10(1), 229. 
Yang, W., Wittkopp, T. M., Li, X., Warakanont, J., Dubini, A., Catalanotti, C., ... Grossman, A. R. (2015). Critical role of Chlamydomonas reinhardtii ferredoxin-5 in maintaining membrane structure and dark metabolism. Proceedings of the National Academy of Sciences of the United States of America, 112(48), 14978-14983.

Yao, Y., Lu, Y., Peng, K. T., Huang, T., Niu, Y. F., Xie, W. H., Yang, W. D., Liu, J. S., \& Li, H. Y. (2014). Glycerol and neutral lipid production in the oleaginous marine diatom Phaeodactylum tricornutum promoted by overexpression of glycerol-3-phosphate dehydrogenase. Biotechnology for Biofuels, 7(1), 110.

Yi, Z., Xu, M., Magnusdottir, M., Zhang, Y., Brynjolfsson, S., Fu, W., \& MartinJézéquel, V. (2015). Photo-oxidative stress-driven mutagenesis and adaptive evolution on the marine diatom Phaeodactylum tricornutum for enhanced carotenoid accumulation. Marine Drugs, 13(10), 6138-6151.

Yohn, C., Mendez, M., Behnke, C., \& Brand, A. (2011). Stress-induced lipid trigger. US Patent No 20,120,322,157 Washington, DC: U.S. Patent and trademark office.

Yoneda, K., Yoshida, M., Suzuki, I., \& Watanabe, M. M. (2018). Homologous expression of lipid droplet protein-enhanced neutral lipid accumulation in the marine diatom Phaeodactylum tricornutum. Journal of Applied Phycology, 30(5), 2793-2802.

Yoon, K., Han, D., Li, Y., Sommerfeld, M., \& Hu, Q. (2012). Phospholipid:diacylglycerol acyltransferase is a multifunctional enzyme involved in membrane lipid turnover and degradation while synthesizing triacylglycerol in the unicellular green microalga Chlamydomonas reinhardtii. The Plant Cell, 24(9), 3708-24.

Yu, J., Loh, K., Song, Z., Yang, H., Zhang, Y., \& Lin, S. (2018). Update on glycerol-3-phosphate acyltransferases: the roles in the development of insulin resistance. Nutrition \& Diabetes, 8(1), 34.

Yu, W. L., Ansari, W., Schoepp, N. G., Hannon, M. J., Mayfield, S. P., \& Burkart, M. D. (2011). Modifications of the metabolic pathways of lipid and triacylglycerol production in microalgae. Microbial Cell Factories, 10, 91.

Zárate, R., Jaber-Vazdekis, N., Tejera, N., Pérez, J. A., \& Rodríguez, C. (2017). Significance of long chain polyunsaturated fatty acids in human health. Clinical and Translational Medicine, 6(1), 25.

Zäuner, S., Jochum, W., Bigorowski, T., \& Benning, C. (2012). A cytochrome b5-containing plastid-located fatty acid desaturase from Chlamydomonas reinhardtii. Eukaryotic Cell, 11(7), 856-863.

Zhang, C., \& Liu, P. (2017). The lipid droplet: A conserved cellular organelle. 
Protein \& Cell, 8(11), 796-800.

Zhang, J., Hao, Q., Bai, L., Xu, J., Yin, W., Song, L., ... Hu, Z. (2014). Overexpression of the soybean transcription factor GmDof4 significantly enhances the lipid content of Chlorella ellipsoidea. Biotechnology for Biofuels, 7(1), 128.

Zhang, S., He, Y., Sen, B., Chen, X., Xie, Y., Keasling, J. D., \& Wang, G. (2018). Alleviation of reactive oxygen species enhances PUFA accumulation in Schizochytrium sp. through regulating genes involved in lipid metabolism. Metabolic Engineering Communications, 6, 39-48.

Zhang, Y. T., Jiang, J. Y., Shi, T. Q., Sun, X. M., Zhao, Q. Y., Huang, H., \& Ren, L. J. (2019). Application of the CRISPR/Cas system for genome editing in microalgae. Applied microbiology and biotechnology, 103(8), 3239-3248.

Zhu, B.-H., Zhang, R.-H., Lv, N.-N., Yang, G.-P., Wang, Y.-S., \& Pan, K.-H. (2018). The role of malic enzyme on promoting total lipid and fatty acid production in Phaeodactylum tricornutum. Frontiers in Plant Science, 9, 826.

Zhu, B. H., Tu, C. C., Shi, H. P., Yang, G. P., \& Pan, K. H. (2017). Overexpression of endogenous delta- 6 fatty acid desaturase gene enhances eicosapentaenoic acid accumulation in Phaeodactylum tricornutum. Process Biochemistry, 57, 43-49.

Zhu, Z., Yuan, G., Fan, X., Fan, Y., Yang, M., Yin, Y., Liu, J., Liu, Y., Cao, X., Tian, J., \& Xue, S. (2018). The synchronous TAG production with the growth by the expression of chloroplast transit peptide-fused ScPDAT in Chlamydomonas reinhardtii. Biotechnology for Biofuels, 11(1), 156.

Zienkiewicz, K., Zienkiewicz, A., Poliner, E., Du, Z. Y., Vollheyde, K., Herrfurth, C., Marmon, S., Farré, E. M., Feussner, I., \& Benning, C. (2017). Nannochloropsis, a rich source of diacylglycerol acyltransferases for engineering of triacylglycerol content in different hosts. Biotechnology for Biofuels, 10(1), 8.

Zou, L. G., Chen, J. W., Zheng, D. L., Balamurugan, S., Li, D. W., Yang, W. D., Liu, J. S., \& Li, H. Y. (2018). High-efficiency promoter-driven coordinated regulation of multiple metabolic nodes elevates lipid accumulation in the model microalga Phaeodactylum tricornutum. Microbial Cell Factories, $17(1), 54$.

Zulu, N. N., Popko, J., Zienkiewicz, K., Tarazona, P., Herrfurth, C., \& Feussner, I. (2017). Heterologous co-expression of a yeast diacylglycerol acyltransferase (ScDGA1) and a plant oleosin (AtOLEO3) as an efficient tool for enhancing triacylglycerol accumulation in the marine diatom Phaeodactylum tricornutum. Biotechnology for Biofuels, 10(1), 187. 
Zulu, N. N., Zienkiewicz, K., Vollheyde, K., \& Feussner, I. (2018). Current trends to comprehend lipid metabolism in diatoms. Progress in Lipid Research, 70, 1-16. 
References 

Acknowledgments 
With this final section, after a 6-years journey this thesis has been finally completed. My personal and professional formation has been only possible thanks to the help and support of friends, colleagues and family for whom I would like to express my gratitude.

I would like to start with my supervisors who made this journey possible. Mark S. and René, I would like to thank you for giving me the opportunity to start my PhD. Thank you for believing in me, even when you knew very little from this small Chilean, who could barely speak English that was coming from very far away. Packo, even though you were my supervisor for a very short period of time, I would like to thank you for your help and supervision. I really appreciated your genuine interest on the project and in my personal development. I will never forget the good times we had during the microalgae process design courses. Ruud, you became my supervisor later on the road. You were continuously challenging me and made me become a better presenter, writer and by following your guidance a lot better at making figures. You definitely made me grow not only as a professional but also at a personal level. You had a great impact in my career and I would like to thank you for always being present and always having some time to talk even when you were passing through tough times. I really admire your commitment, genuine interest and dedication for your students and colleagues. Sarah, although you arrived at the end of my $\mathrm{PhD}$, you were essential for finishing my thesis. Always available, always willing to help and have a chat during my ups and downs. I consider you a great person and I am very grateful to have spent time with you at work, PhD trip and BBQs.

I would also like to thank the students I have supervised throughout the years. Marina, Martim, Iris, Miquel, Marc and Nick you were definitely very important for the completion of my thesis. You have taken the challenge, you have showed motivation and perseverance and thus, I have no doubt that you will succeed in life. Thank you for your hard work, I wish you great success in your future careers. 
All of this work would have not been possible without the support, help and hard work of BPE staff members. Miranda, Marina, Snezana, Wendy, Fred, Sebastiaan and Rick, I would like to thank you for your crucial role in administrative and technical tasks, you were always willing to help and made my life a lot easier, I really appreciate it. I also would like to thank staff members: Rene, Maria, Dirk, Packo, Rafa, Iago, Iris, Iulian, Michel, Arjen, Giuseppe, Marcel, Sarah, Ruud, Mark B., Hans, Antoinette and Corjan. Although you were not all directly involved in my project, in one way or another you help in my personal development. It has been a real pleasure to spend time with all of you at BPE, group meetings, dinners, labuitjes and other social events.

Over the years my office E.2.131 became some sort of a compulsory space for new people coming to BPE. I had the pleasure to share my office with incredible people: Catalina, Joao, Robin, Anna, Ana P., Rocca, Bárbara, Rupali, Maria V. and Elisa. Although I am not a very talkative and at times, I might seem like a "scary" officemate, I really enjoyed the time we spent together and I would like to thank you all for being part of this journey.

I would like to thank all Dinners/Beers and BestPeopleEver colleagues and friends: Jort, Calvin, Kylie, Narcís, Nicola, Fabian, Pieter, August, Chunzhe, Jin, Enrico, Iulian, Anna, Nuran, Sabine, Antoinette, Pedro, Robin, Bárbara, Ana, Christian, Sebastian, Renske, Rafa C., Marta, Wendy, Edgar, Iris and Youri. Thank you all for making life after work fun, I will always remember the amazing trips, dinners, BBQs and other activities we got to have together. Enrico, "el weon", thank you for the amazing times. It was always great to have you around either for a chess match, dinners where you always showcase your Italian skills in the kitchen specially with some fresh pasta, football matches, always sharing funny stories or imitating other people, and of course always present when it came to talk about science. Fabian, I will always remember the fun times, dinners and BBQs at your place and the great time we spent in the PhD trip. Having drinks with you was always an unforgettable experience which always ended up being a funny story to tell. Narcís, the captain of our BPE football team. Thank you for always pushing me 
to do more sports and to challenge my limits. For sure, I would have gained a lot more weight if it was not for the encouragement. I have really enjoyed all the unforgettable BBQs, dinners and even breakfast we had at your place. Thank you very much for always being there in the very important moments, especially when you became my witness during my register partnership with Pauline. Anna thanks for the fun times, countless dinners, drinks, BBQs, Christmas markets, thank you for making me and Pauline feel welcome at your place in The Netherlands and Germany. Alex, I will never forget the all the trips, drinking sessions, the climbing and the time we lived together when I got to enjoy your amazing cooking. Thank you for always bringing fun and for welcoming me in your place in the beautiful Slovenia. Ana $\mathbf{P}$, although we did not spend a lot of time together, I have very good memories. Thanks for the nice talks at the office, meals at Narcís place where you showed your great skills in Spanish food, and of course our bicycle trip and movie nights with the guys. August and Jin, it has been always very nice to talk to you, I will never forget our dumpling dinners. Jort and Chunzhe I enjoyed a lot having drinks with you and it has been great to talk about life, science and of course about financial markets. I wish you great success in your future investments.

When I think of old times and beginning of my journey at BPE I always remember: Youri, Iago, Carl, Aziz, Catalina, Jeroen, Agi, Josue, Rafa, Edgar, Luci, Christina, Ward, Joao and Alex. Thank you for making me feel welcome, I will always remember our trips, talks, drinks and BBQs.

Valentina and Alexia, I am very happy to have met you through Pauline. You have been always present in the very important moments and really would like to thank you for your support and all the good times we got to share together throughout the years.

I would like to thank: José, Jacqueline, Lydie, Fabrice, Anna-Rose, Joaquim, Corrine, Philippe, Audrey, Mathieu, Claudy, Evelyne, Maryse, Sandra and Anaïs. I have really enjoyed the time we have spent together during holidays, having incredible food and visiting amazing places in the south 
of France. I have no words to describe how grateful I am for your hospitality and for always make me feel like part of your family.

También quisiera agradecer a mi familia: Solange, Ismael, Martín, Alejandrina, Hugo, Trinidad, Vicente, Modesto, Alejandra, Josefa, Valentina, Miguel, Kenita, Kenia, Kevin, Maira, Norma, Domingo y Elena. Muchas gracias por el apoyo incondicional y el cariño que he sentido a pesar de la distancia y del poco tiempo que nos hemos visto en los últimos años.

Felipe y Benjamín, no saben lo agradecido que soy de tenerlos como hermanos y lo orgulloso que soy de saber que están logrando sus metas en Europa. A pesar de no vernos tan seguido, el saber que siempre puedo contar con ustedes o saber que los veré en mis vacaciones me llena de energía y motivación para continuar con los desafíos que enfrento cada día. Mamá y papá, definitivamente ustedes han sido esenciales para conseguir este logro tan importante en mi vida. Ustedes me han dado no solo el apoyo incondicional, pero también han sido mi fuente de inspiración a lo largo de mi vida. No tengo las palabras suficientes para agradecerles por todos estos años de esfuerzo y sacrificios. Eternamente agradecido y orgulloso de tenerlos como mis padres, los quiero mucho.

Pauline, as you would say, we are becoming Mr. and Ms. Doctor. Your unconditional love, support and friendship have been crucial during this journey. Besides being an incredible scientist, you have become a very important pillar in my life, my best friend and family as well. I am very lucky to have found you and get to share my life with you. 


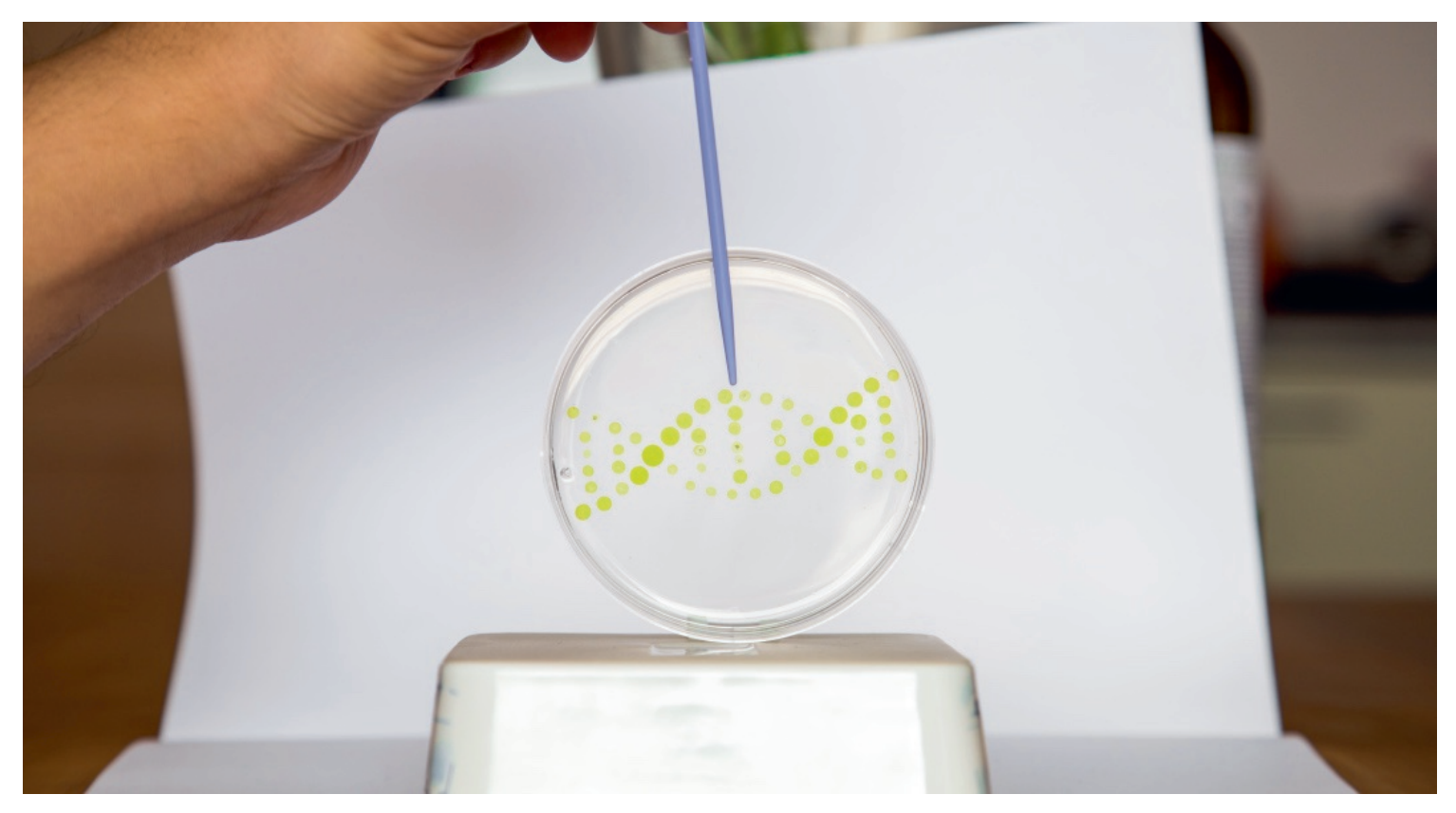


About the author 


\section{About the author}

Camilo Felipe Muñoz Segovia was born on October $6^{\text {th }} 1989$ in Antofagasta, Chile. In 2014 he obtained his BSc degree in Biotechnology at University of Antofagasta. In his BSc thesis he worked on method development for growth/product analysis in photosynthetic microalgae at the University of the Frontier, Temuco (Chile) under the supervision of dr. David Jeison. In 2013, before his graduation, Camilo

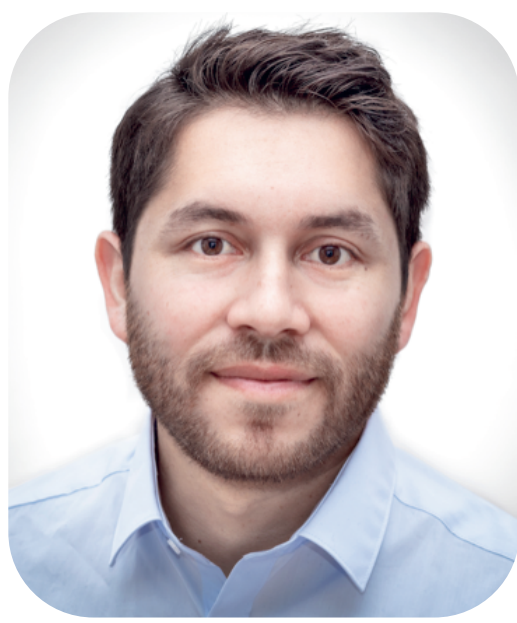
worked as a research assistant for six months at Instituto de Recursos Naturales y Agrobiología de Sevilla (IRNAS) (Spain) in the production of recombinant hydrolytic enzymes from thermophilic microorganisms, for their use as pre-treatment of microalgal biomass. After his BSc graduation, Camilo worked as research assistant at University of Antofagasta (Chile). There, he worked on genetic tool development for genetic engineering of microalgae and screening/characterization of bacterial strains with enhanced enzymatic activity for microalgae biomass pretreatment.

On January $15^{\text {th }}$ 2015, Camilo started his PhD thesis at BPE under the supervision of dr. Mark Sturme, dr. Packo Lammers, dr. Sarah D’adamo, prof. Ruud Weusthuis and prof. René Wijffels. During this project, he worked on genetic tool development and metabolic engineering of microalgae for enhanced lipid production. Since January 2019, Camilo is employed as Postdoctoral researcher at BPE and MIB group. Initially, he worked in thermodynamics and metabolic modeling in the project Bacterial conversion of $\mathrm{CO}_{2}$ and renewable $\mathrm{H}_{2}$ into biofuels (Bac-To-Fuel). Currently, he works as BSc and MSc thesis supervisor within the microbial biotechnology team at BPE. 


\section{List of publications}

Muñoz, C.F., de Jaeger, L., Sturme, M.H.J., Lip, K.Y.F., Olijslager, J.W.J., Springer, J., Wolbert, E.J.H., Martens, D.E., Eggink, G., Weusthuis, R.A., Wijffels, R.H. (2018). Improved DNA/protein delivery in microalgae - A simple and reliable method for the prediction of optimal electroporation settings. Algal Research, 33: 448-455.

Muñoz, C.F., Sturme, M.H.J., D'Adamo, S., Weusthuis, R.A., Wijffels, R.H. (2019). Stable transformation of the green algae Acutodesmus obliquus and Neochloris oleoabundans based on E. coli conjugation. Algal Research, 39: 101453.

Muñoz, C.F., Weusthuis, R.A., D'Adamo, S., Wijffels, R.H. (2019). Effect of single and combined expression of LPAT, GPAT and DGAT on lipid accumulation and composition in Neochloris oleoabundans. Frontiers in Plant Science, 10: 1573.

Muñoz, C.F., Südfeld, C., Naduthodi, M.I.S., Weusthuis, R.A., Barbosa, M.J., Wijffels, R.H. D'Adamo, S. (2021). Genetic engineering of microalgae for enhanced lipid production. Biotechnology Advances, 52: 107836. 


\section{Overview of completed training activities}

\section{Discipline specific activities}

- Microalgae process design: from cells to photobioreactors, The Netherlands (2015)

- Metabolic engineering 12, Germany (2018)

- AlgaeEurope, The Netherlands (2018)

- Microbial biotechnology 6.0, The Netherlands (2019)

- Netherlands Biotechnology Congress, The Netherlands (2021)

\section{Optional activities}

- Research proposal, The Netherlands (2015)

- PhD trip, USA (2018)

- BPE group meetings, The Netherlands (2015-2019)

- Theme meeting microalgal biotechnology, The Netherlands (2017-2019)

- Microbial biotechnology theme meeting, The Netherlands (2017-2019)

- Meeting algae strain development, The Netherlands (2017-2019)

- Metabolic engineering tools meeting, The Netherlands (2015-2017)

\section{MSc Courses}

- Genetic analysis trends and concepts, The Netherlands (2016)

- Metabolic engineering of industrial microorganisms, The Netherlands (2016) 
The research described in this thesis was financially supported by the National Commission of Scientific and Technologic Research of Chile (CONICYT).

Cover design by Camilo F. Muñoz Segovia

Printed by Digiforce || ProefschriftMaken 


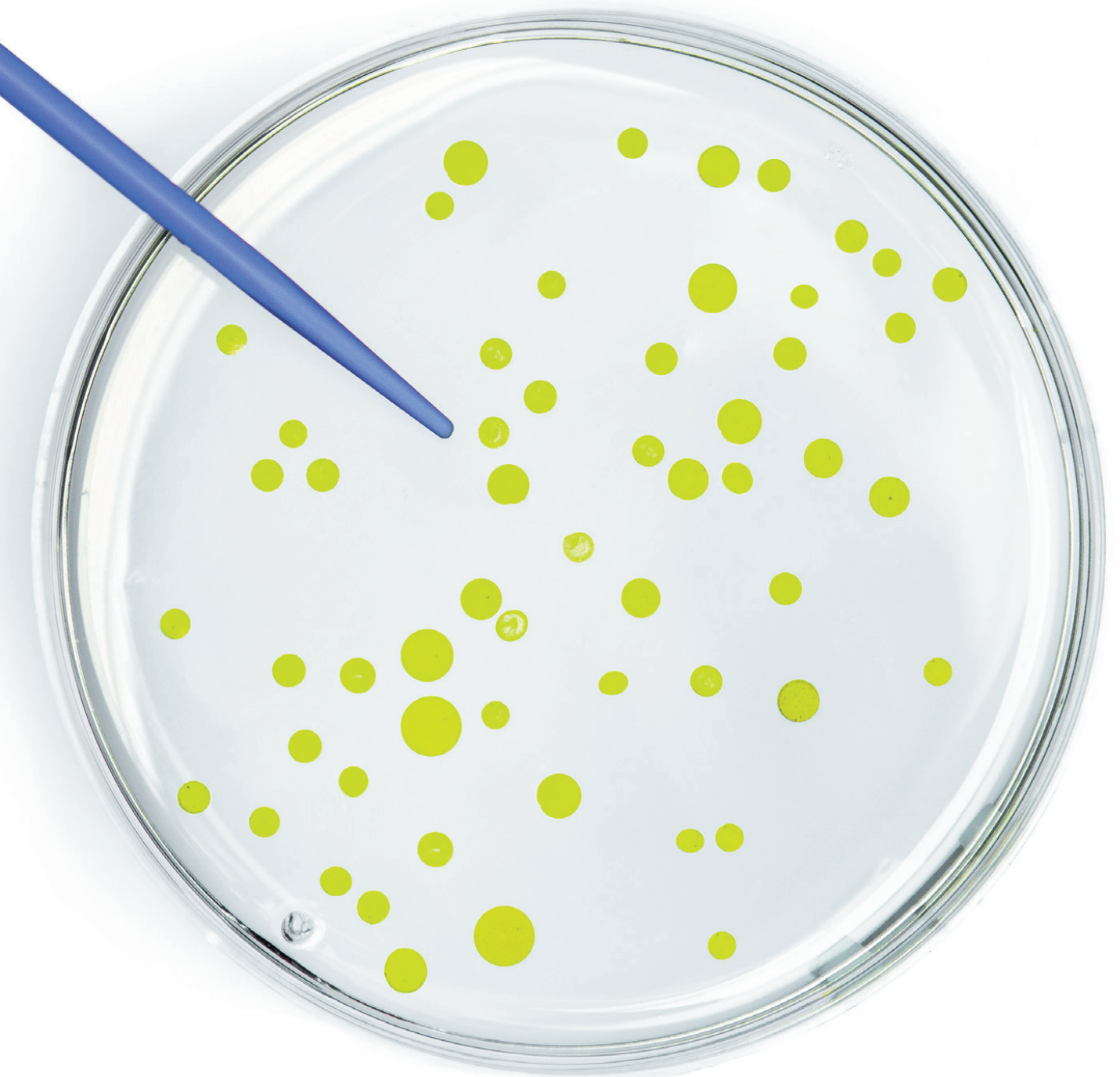

